\title{
Navigation of Mobile Robots using Occupancy Grids
}

by

\author{
Mitul Ashwin Adhiya \\ B.Engg
}

\begin{abstract}
A thesis submitted to the Faculty of Graduate Studies and Research
in partial fulfillment of the requirements for the degree of

Master of Applied Science
\end{abstract}

Ottawa-Carleton Institute for Mechanical and Aerospace Engineering

\author{
Department of Mechanical and Aerospace Engineering \\ Carleton University \\ Ottawa, Ontario \\ Canada
}

April, 2007

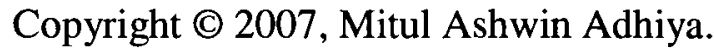




$\begin{array}{ll}\begin{array}{l}\text { Library and } \\ \text { Archives Canada }\end{array} & \begin{array}{l}\text { Bibliothèque et } \\ \text { Archives Canada }\end{array} \\ \begin{array}{l}\text { Published Heritage } \\ \text { Branch }\end{array} & \begin{array}{l}\text { Direction du } \\ \text { Patrimoine de l'édition }\end{array} \\ \begin{array}{l}\text { 395 Wellington Street } \\ \text { Ottawa ON K1A 0N4 }\end{array} & \begin{array}{l}\text { 395, rue Wellington } \\ \text { Ottana ON K1A ON4 } \\ \text { Canada Oa }\end{array}\end{array}$

Your file Votre référence ISBN: 978-0-494-26980-0 Our file Notre référence ISBN: 978-0-494-26980-0

NOTICE:

The author has granted a nonexclusive license allowing Library and Archives Canada to reproduce, publish, archive, preserve, conserve, communicate to the public by telecommunication or on the Internet, loan, distribute and sell theses worldwide, for commercial or noncommercial purposes, in microform, paper, electronic and/or any other formats.

The author retains copyright ownership and moral rights in this thesis. Neither the thesis nor substantial extracts from it may be printed or otherwise reproduced without the author's permission.
AVIS:

L'auteur a accordé une licence non exclusive permettant à la Bibliothèque et Archives Canada de reproduire, publier, archiver, sauvegarder, conserver, transmettre au public par télécommunication ou par l'Internet, prêter, distribuer et vendre des thèses partout dans le monde, à des fins commerciales ou autres, sur support microforme, papier, électronique et/ou autres formats.

L'auteur conserve la propriété du droit d'auteur et des droits moraux qui protège cette thèse. $\mathrm{Ni}$ la thèse ni des extraits substantiels de celle-ci ne doivent être imprimés ou autrement reproduits sans son autorisation.
In compliance with the Canadian

Privacy Act some supporting forms may have been removed from this thesis.

While these forms may be included in the document page count, their removal does not represent any loss of content from the thesis.
Conformément à la loi canadienne sur la protection de la vie privée, quelques formulaires secondaires ont été enlevés de cette thèse.

Bien que ces formulaires aient inclus dans la pagination, il n'y aura aucun contenu manquant. 
The undersigned recommend to

the Faculty of Graduate Studies and Research acceptance of the thesis

\title{
Navigation of Mobile Robots using Occupancy Grids
}

\author{
Submitted by \\ Mitul Ashwin Adhiya \\ B.Engg \\ in partial fulfillment of the requirements for \\ the degree of \\ Master of Applied Science
}

Thesis Supervisor

Chair, Dept. of Mechanical and Aerospace Engineering

Carleton University

April 2007 


\begin{abstract}
A local navigation method for autonomous mobile robots with obstacle avoidance is developed in which the dynamics of the robot are considered. Car-like robots are mobile robots with dynamics and kinematics of a car, operating for specific transportation task in indoor and outdoor unknown environment may have to follow pre-defined trajectory or build a map and design trajectory as they travel and have to avoid obstacles discovered on their path during the operation. The only information required about the local environment is the distance between the robot and the angle made by obstacles with respect to robot's frame. Mapping is carried out using occupancy grid method in which the workspace of the robot is divided into square grids and each one is allocated with probability. Based on these probabilities, the occupancy of grids with obstacles is determined. Localization and estimating range and azimuth angle obtained from laser range sensor is carried out using Extended Kalman Filter (EKF). Since localization and simultaneously mapping of the environment is carried out for local path planning, the problem of localization and mapping can also be categorized under Simultaneous localization and Mapping (SLAM). Finally, obstacle avoidance algorithm is developed to fulfill these required tasks. Obstacle avoidance should be done in a way that robot does not diverge much away from the trajectory. Also, after avoiding obstacle it needs to come back on trajectory considering minimum distance. The effectiveness of the technique is demonstrated by means of simulation software examples in 2D environment.
\end{abstract}




\section{Acknowledgements}

I would like to sincerely thank my thesis supervisor Professor J. Z. Sasiadek for his guidance, support and motivation throughout this thesis. He has constantly been a great source of knowledge to me and I am highly grateful to him.

I would like to thank all the staff members of department of mechanical \& Aerospace engineering for their various supports and $\mathrm{Mr}$. Yi Lu for his precious help for LMS program development.

I heartily thank my beloved parents for their moral and financial support and my sisters Kanan and Aparna who always gave me encouragement. 


\section{Table of Contents}

$\begin{array}{lr}\text { Abstract } & \text { iii } \\ \text { Acknowledgement } & \text { iv } \\ \text { Table of Contents } & \text { viii } \\ \text { List of Figures } & \text { xii } \\ \text { List of Tables } & \text { xii } \\ \text { Nomenclature } & \end{array}$

CHAPTER 1: THESIS INTRODUCTION AND OBJECTIVES 1

CHAPTER 2: WHEELED MOBILE ROBOTS 9

2.1 Introduction 9

2.2 Holonomic constraints 12

2.3 Nonholonomic constraints 13

2.4 Motion control tasks for car-like robots 16

2.5 Modeling and analysis of car-like robot 20

CHAPTER 3: INTRODUCTION AND LITERATURE REVIEW FOR MOBILE ROBOT LOCALIZATION 26

3.1 Introduction 26

3.2 Bayesian algorithm for mobile robot localization 27

3.3 Kalman filters 29

3.3.1 Discrete Kalman filter 30

3.3.2 Extended Kalman filter 33

3.4 Dead reckoning for localization 35

3.5 Implementation of mobile robot localization 39 
CHAPTER 4: INTRODUCTION AND LITERATURE REVIEW FOR MAPPING 46

4.1 Introduction 46

4.2 Topological representations $\quad 48$

4.2.1 Visibility graphs 48

4.2.2 Generalized Voronoi diagrams or retraction approach $\quad 51$

4.3 Geometric representation $\quad 52$

4.4 Occupancy grids representations 53

4.5 Brief comparison between topological and grid based methods 55

4.6 Occupancy grid development for mobile robot navigation 56

CHAPTER 5: PATH-TRACKIG FOR CAR-LIKE ROBOT 64

$5.1 \quad$ Background of path-tracking for car-like robot 64

5.2 Kinematics and dynamics concepts 66

5.3 Model development of car-like robot 68

$\begin{array}{lll}5.4 & \text { Path-tracking controllers design } & 71\end{array}$

CHAPTER 6: COLLSION AVOIDANCE ALORITHM 84

$\begin{array}{lll}6.1 & \text { Background } & 84\end{array}$

6.1.1 Potential field 85

6.1.2 Bug algorithm 86

$\begin{array}{lll}6.2 & \text { Collision avoidance design } & 87\end{array}$

6.2.1 Collision detection 89

6.2.2 Selection of obstacle end point 102

6.2.3 Selection of target point 105

6.2.4 Determination of steering angle 109

6.2.5 Presence of close obstacles 112

$\begin{array}{lll}\text { 6.2.6 Steering controller } & 114\end{array}$

CHAPTER 7: SIMULATIONS AND RESULTS 115

$\begin{array}{lll}7.1 & \text { Simulation background } & 115\end{array}$ 
$\begin{array}{lll}7.2 & \text { Simulation } & 118\end{array}$

7.2.1 Initialization 118

7.2.2 Dynamics and kinematics models 119

7.2.3 Localization using Extended Kalman filter 120

7.2.4 Trajectory tracking 129

7.2.5 Scanning and mapping environment 136

7.2.6 Obstacle avoidance and steering angle determination 141

CHAPTER 8: CONCLUSION AND FUTURE WORK 159

$\begin{array}{lll}8.1 & \text { Conclusions } & 159\end{array}$

$\begin{array}{lll}8.2 & \text { Future work } & 161\end{array}$

$\begin{array}{ll}\text { REFERENCES } & 163\end{array}$

APPENDICES

1. Algorithm for the flow of simulation 170

2. Simulation program code in MATLAB 171

vii 


\section{List of Figures}

1.1 Robot, task and environment interdependency 2

$2.1 \quad$ Examples of WMRs 10

$\begin{array}{lll}2.2 & \text { Car-like robot } & 13\end{array}$

2.3 Velocity vector of car-like robot whose turning radius is bounded by $\rho_{\min } \quad 16$

$\begin{array}{lll}2.4 & \text { Point-to-point motion problem } & 17\end{array}$

2.5 Stabilization at equilibrium manifold: path following 18

2.6 Stabilization at equilibrium manifold: Trajectory tracking 19

$2.7 \quad$ Unicycle 21

2.8 Car-like wheeled mobile robot. 23

3.1 Steps for discrete Kalman filter 33

3.2 Typical differential-drive mobile robot 36

3.3 Distance traveled on curved path 37

3.4 Accumulation of odometry errors with distance traveled 38

3.5 Dead reckoning geometry 41

3.6 Errors in states X, Y and Theta 42

3.7 States updated using EKF with $R=500 I_{3}$ and $Q=10 I_{3}$.

3.8 Difference between updated and predicted states, $R=200 I_{3}$ and $Q=10 I_{3} \quad 45$

$\begin{array}{lll}4.1 & \text { Polygonal configuration in space } & 49\end{array}$

$\begin{array}{lll}4.2 & \text { Visibility graphs } & 49\end{array}$

4.3 Generalized Voronoi diagram 52

4.4 Occupancy probability map of an office environment built from sonar measurements 
4.5 Generation of occupancy grid 63

$\begin{array}{lll}5.1 & \text { Car-like robot } & 69\end{array}$

$\begin{array}{lll}5.2 & \text { Step response of propulsion controller } & 76\end{array}$

$\begin{array}{lll}5.3 & \text { Block diagram of steering controller } & 77\end{array}$

$5.4 \quad$ Open loop time response of error model. $\quad 78$

5.5 Path tracking on straight path with $C_{1}=10$ and $C_{2}=1$

5.6 Distance offset and orientation offset for $C_{1}=10$ and $C_{2}=1$

5.7 Path tracking on curved path with $C_{1}=10$ and $C_{2}=1$.

$\begin{array}{llr}6.1 & \text { Structure of collision avoidance } & 88\end{array}$

6.2 Collision zone (units is meter) 90

6.3 One detection case $\quad 91$

6.4 Collision area on either side of longitudinal axis 95

6.5 Two detection single object 96

6.6 Two detection multiple objects. $\quad 98$

$\begin{array}{lll}6.7 & \text { Three detection case } & 101\end{array}$

$\begin{array}{lll}6.8 & \text { Selection of obstacle end point } & 103\end{array}$

6.9 Selection of obstacle point with goal behind object 105

6.10 Selection of target point when collision is detected 106

6.11 Different situations for selection of goal point on trajectory. 108

6.12 Robots orientation in different quadrants 110

$\begin{array}{ll}\text { 6.13 Collision avoidance of close obstacles } & 113\end{array}$

$\begin{array}{lll}7.1 & \text { Flowchart for MATLAB simulation } & 117\end{array}$

$\begin{array}{lll}7.2 & \text { Dynamics and kinematics } & 119\end{array}$ 
7.3 Updated location with $\mathrm{Q}=1 \mathrm{I}_{3} \mathrm{R}=50 \mathrm{I}_{3}$

7.4 Updated orientation with $Q=1 I_{3} R=50 I_{3}$

7.5 Updated location with $\mathrm{Q}=1 \mathrm{I}_{3} \mathrm{R}=200 \mathrm{I}_{3}$

7.6 Updated orientation with $Q=1 I_{3} R=200 I_{3}$

7.7 Updated location with $\mathrm{Q}=1 \mathrm{I}_{3} \mathrm{R}=500 \mathrm{I}_{3}$

7.8 Updated orientation with $Q=1 I_{3} R=500 I_{3}$

7.9 Updated location with $\mathrm{Q}=1 \mathrm{I}_{3} \mathrm{R}=700 \mathrm{I}_{3}$

7.10 Updated orientation with $Q=1 I_{3} R=700 I_{3}$ 124

7.11 Updated location with $\mathrm{Q}=10 \mathrm{I}_{3} \mathrm{R}=50 \mathrm{I}_{3}$

7.12 Updated orientation with $Q=10 I_{3} R=50 I_{3}$

7.13 Updated location with $\mathrm{Q}=10 \mathrm{I}_{3} \mathrm{R}=200 \mathrm{I}_{3}$ 125

7.14 Updated orientation with $Q=10 I_{3} R=200 I_{3}$

7.15 Updated location with $\mathrm{Q}=10 \mathrm{I}_{3} \mathrm{R}=500 \mathrm{I}_{3}$

7.16 Updated orientation with $Q=10 \mathrm{I}_{3} \mathrm{R}=500 \mathrm{I}_{3}$

7.17 Updated location with $\mathrm{Q}=10 \mathrm{I}_{3} \mathrm{R}=700 \mathrm{I}_{3}$

7.18 Updated orientation with $\mathrm{Q}=10 \mathrm{I}_{3} \mathrm{R}=700 \mathrm{I}_{3} \quad 128$

7.19 Trajectory tracking and offsets for $C_{1}=1$ and $C_{2}=50$. 130

7.20 Trajectory tracking and offsets for $C_{1}=1$ and $C_{2}=1$. 132

7.21 Trajectory tracking and offsets for $C_{1}=20$ and $C_{2}=10$. 133

7.22 Trajectory tracking and offsets for curved path with $C_{1}=1$ and $C_{2}=50$. 133

7.23 Trajectory tracking and offsets for curved path with $C_{1}=1$ and $C_{2}=1$. 134

7.24 Trajectory tracking and offsets for curved path with $C_{1}=20$ and $C_{2}=10 \quad 135$

7.25 Local scan view using LMS for one obstacle. Units are in meters. 138 
7.26 Local scan view using LMS for one obstacle.

7.27 Local scan view using LMS for two obstacles.

7.28 Local scan view of LMS after filtering data within maximum range.

7.29 Grids accounted for single sweep of LMS (a) and occupied grids (b)

7.30 Collision avoidance with one object, one detection on right off trajectory

7.31 Collision avoidance with one object, two detection on left and front off trajectory

7.32 Collision avoidance with one object, all three detection off trajectory

7.33 Presence of object from 2 meters away from trajectory.

7.34 Presence of object on the trajectory

7.35 Obstacle detection from 20 meters

7.36 Obstacle detection from 15 meters

7.37 Two obstacles detection with robot moving on trajectory

7.38 Two obstacles detection with robot away from trajectory

7.39 Three obstacles detection with robot away from trajectory

7.40 Gate with robot away from trajectory

7.41 Tunnel with robot on trajectory 


\section{List of Tables}

3.1 Selection for Q and R. $\quad 43$

4.1 Advantages and disadvantages of grid based and topological approaches 56

$5.1 \quad$ LQR controller parameters $\quad 80$

xii 


\section{Nomenclature}

\begin{tabular}{|c|c|}
\hline$(x, y)$ & Robot's reference point configuration on $2 \mathrm{D}$ plane \\
\hline$\theta$ & Robot's orientation with respect to reference $\mathrm{X}$ axis \\
\hline$q$ & Vector of generalized coordinates \\
\hline$a_{i}$ & Holonomic constraints \\
\hline$(\dot{x}, \dot{y})$ & Velocities in $\mathrm{x}$ and $\mathrm{y}$ direction \\
\hline$\omega$ & Angular velocity \\
\hline$\delta$ & Steering angle \\
\hline$\rho_{\min }$ & Minimum turning radius \\
\hline$A$ & System dynamic matrix \\
\hline${ }^{\mathbf{w}_{k-1}}$ & System noise at $(\mathrm{k}-1)^{\text {th }}$ step \\
\hline${ }^{\mathbf{v}_{k}}$ & Sensor noise at $\mathrm{k}^{\text {th }}$ step \\
\hline$\varphi_{k}$ & State transition matrix \\
\hline $\mathbf{Q}_{k}$ & System noise covariance matrix \\
\hline $\mathbf{R}_{k}$ & Measurement noise covariance matrix \\
\hline $\mathbf{P}_{k}$ & Error covariance matrix \\
\hline$v_{u}$ & Longitudinal velocity \\
\hline$v_{w}$ & Lateral velocity \\
\hline
\end{tabular}




\begin{tabular}{|c|c|}
\hline$v_{d}$ & Desired robot velocity \\
\hline$p($ cell $=$ occ $)$ & Probability of grid cell to be occupied with obstacle \\
\hline$m_{x, y}$ & Occupancy of the grid cell with index $\langle x, y\rangle$ \\
\hline$r_{1}, r_{2}, \ldots, r_{t}$ & Range readings from laser range sensor \\
\hline$\sigma_{r}$ & Range standard deviation \\
\hline$\sigma_{\theta}$ & Azimuth angle standard deviation \\
\hline$l_{x, y}$ & Log-odds \\
\hline $\mathbf{J}_{h}$ & Holonomic constraints matrix \\
\hline $\mathbf{J}_{n h}$ & Non-holonomic constraints matrix \\
\hline$q_{d}(c)$ & Desired configuration \\
\hline$v_{o s}=v_{u}-v_{u d}$ & Velocity offset \\
\hline$\theta_{o s}=\theta-\theta_{d}$ & Orientation offset \\
\hline$\delta_{o s}=\delta-\delta_{d}$ & Steering angle offset \\
\hline \multicolumn{2}{|c|}{$l_{o s}=-\left(x-x_{d}\right) \sin \theta_{d}+\left(y-y_{d}\right) \cos \theta_{d} \quad$ Lateral offset } \\
\hline$F_{s}$ & Steering control input \\
\hline$F_{p}$ & Propulsion control input \\
\hline $\mathbf{K}_{s}$ & Steering angle controller gain \\
\hline$K_{p}$ & Propulsion controller gain \\
\hline EKF & Extended Kalman Filter \\
\hline
\end{tabular}


GPS

LQR

SLAM

VPH

VFH

WMRs

Global Positioning System

Linear Quadratic Regulator

Simultaneous Localization And Mapping

Vector Polar Histogram

Vector Field Histogram

Wheeled Mobile Robots 


\section{Chapter 1: Thesis introduction and objectives}

\section{Thesis introduction:}

The word "robot" stems from the play from 1921 called "Rossum's Universal Robots" by Czech playwright Karel Capek. Capek derived the word "robot" from the Czech "robota", meaning "forced labours". In 1942 the word "robotics" appeared for the first time in a novel called "Runaround" by the American scientist and writer Isaac Asimov. A robot is a re-programmable, multi-functional manipulator designed to perform different tasks such as to move material, parts, tools, or specialized devices through programmed motions. Mobile robot considered here have certain degree of autonomy that it should be:

- Able localize itself in environment.

- Able to move in workspace to perform specific task.

- Able to understand and build environment model through sensor outputs for navigation.

- Capable of avoiding obstacles and reach the goal point.

- Capable to adapt to the changes in its environment.

- Capable of learning from experience and change its behavior accordingly.

A robot's function and operation are defined by the robot's own behavior within a specific environment, taking into considering specific task. This interlink is shown in figure below and cannot be considered independent from one another. 


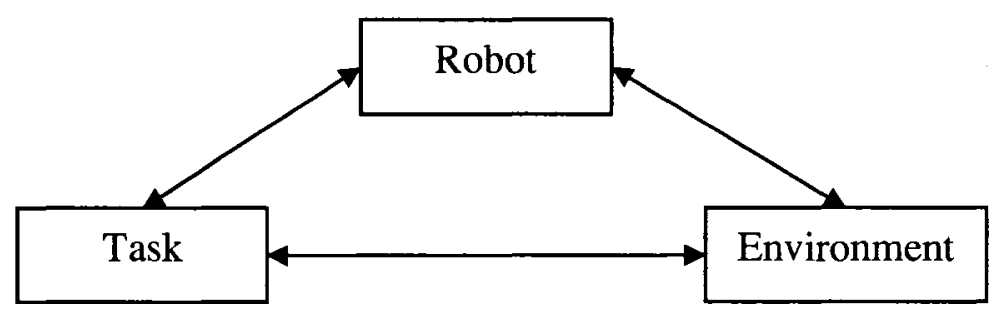

\section{Figure 1.1: Robot, task and environment interdependency}

The easiest way to guide the mobile robot to reach particular location is to guide it. This is usually done by burying inductive loop or magnets in floor, painting lines, or by placing beacons, barcodes, etc for both indoor and outdoor applications. In general, mobile robot should be given a reference trajectory that should take it to the goal position. There are many methods to generate reference trajectories but the one that takes into consideration kinematic and dynamic constraints should be considered. The usual practice to navigate is path integration using dead reckoning. Autonomous vehicles are used in industrial scenarios for transportation tasks, can carry thousands of payloads and are expensive to install or modify after installations, which requires specially prepared environment. But in cases where the environment is not fixed like mining or some planetary environment with no prior knowledge of an environment, it is desirable to build robot navigation system that would allow a mobile robot to navigate in unmodified or natural environments. The ability to navigate is one of the most important capabilities of all. But at the same time, the detection of obstacle and its avoidance is also important while navigating to the goal. Hence, there should be sound integration between navigation and obstacle avoidance to carry out successful navigation task. Navigation can be defined as the combination of the three fundamental competences: 
1) Localization

2) Path planning.

3) Map building

For any particular task of mobile robot, the fundamental question that arises for the robot is "Where am I?" before making decision regarding the actions or actuations for the following step. To answer this question, data from the sensors should be accurately interpreted after filtering out the noise from it. Various methods have been developed for estimating the current location or to update states based on Bayesian estimation theory. After acquiring the knowledge of current location, second question arises as "Where do I go?" Given the partial knowledge of environment and the goal position or series of positions, path planning encompasses the ability of the robot to act, based on its knowledge and location values so as to reach its goal positions as efficiently and reliably as possible.

Path planning is a strategic problem solving competence, as the robot must decide what to do over the long term to achieve its goals. The third question comes "How do I reach the goal without collision?" In some cases, the environment within which robot is moving is not known, like some planets or underground mining operations, it needs to generate the map based on which path planner decides its further plan of action. Even when the prior knowledge of environment is available, in some cases it never remains constant as time progresses or during the course of operation. For example, in case of mines though maps may be available but unexpected presence of workers or any activity done that causes changes in environment such as placing of lump of sand on the way of robot or 
presence of some other mining vehicles are not accounted in available maps. Being autonomous, mobile robots must take into account these uncertainties. For this, at every stage of operation it has to generate the map using information from exteroceptive sensors, such as laser range finders, sonar, etc. Different methods have been developed to generate maps such as topological methods, graphical methods and grid based methods. Grid based (Moravec and Elfes, 1985) method, also known as occupancy grids were found to be most successful among these methods. In addition to path planning, obstacle avoidance plays a major role in navigating mobile robot without collision and to reach goal safely. Several approaches have been developed to handle collision avoidance problem. Local path planner uses this information from map and modifies the path or trajectory as developed during path planning so as to reach goal without encountering collision. Hence if we want to build an autonomous navigating robot that can operate in unmodified or natural environment, all these competences have to be integrated.

Mobile robots with kinematics and dynamics of car, know as car-like mobile robots. They are among one of the most used autonomous robots in industrial tasks, surveillance, planetary exploration for scientific research, etc. They are non linear systems and can be controllable [1] and a class of nonholonomic mechanical systems. During a particular application such as transportation in indoor or outdoor environment, they are provided with reference path satisfying nonholonomic constraints that need to be followed using path tracking controllers. Steering controller is used to control desired steering angle and velocity controller is used to maintain the speed of robot. The process of avoiding obstacles in unknown or partially known environment sometime creates a problem where 
robot travels too far away from the trajectory unnecessarily results to increase in time of operation, reduce the productivity and increase operational costs. In such cases, the robot must be able to avoid obstacle and at the same time it should take into consideration the location of its pre-defined trajectory. The local path planner based on this decides the steering angle that enable robot to reach trajectory as soon as possible. Also, after avoiding obstacle, while coming back on trajectory, the selection of point on the trajectory should be such that it converges with it smoothly.

\section{Thesis objectives}

The objective of this thesis is to improving the navigation efficiency of car-like mobile robot. As previously discussed, different aspects of navigation includes localization, mapping and trajectory tracking. Before making any decision regarding future motion, path planner must know its current location and orientation in the plane in which robot is moving. The sensor readings are always inaccurate due to presence of some noise due to system vibrations, slippage, etc. This will result in inaccurate localization and thus path planner may fail. To avoid this, it necessitates adopting some methodology to filter out this noise, like Extended Kalman Filter (EKF) to estimate the current position and orientation considering nature of noise. Secondly, for successful navigation and to have perception, the robot should have knowledge of the surrounding environment. Number of methods has been developed for mapping using extroceptive sensors such as, sonar, laser, ultrasonic, etc. Because of its advantages, occupancy grid method is used to generate spatial reorientation of environment using laser range finders. The advantages are 
discussed in later chapters. Occupancy grid, developed for spatial representation of environment, uses the data from laser range finder after filtering it using EKF because, there is always a possibility of presence of sensor noise. Also, while performing specific task, the robot may encounter obstacles on its way and it needs to be avoided. During obstacle avoidance, the algorithm must see that the robot does not diverge away from the trajectory which results to increase in operating time and operating costs. Obstacle avoidance algorithm using occupancy grid based environmental representation is to be develop considering location of final goal point, number of obstacles and location of robot with respect to trajectory. In this, the algorithm calculates desired steering angle based on different situations and the error which is difference between current configuration and required configuration is fed to PD controller to control steering angle. Should this occur, it would be an advantageous for path planner to bypass obstacles the without stopping in front of obstacle. To keep running robot on its predefined trajectory, linear quadratic controller is used to control steering angle and the reason for this is discussed in chapter 5 and PI controller for driving with given speed. Differential flatness method is used to generate reference trajectory that takes into consideration nonholonomic constraints of car-like robot. The working environment is considered to be flat. The results of proposed algorithm are shown by MATLAB simulation software considering 2D environment. 
The thesis objectives can be summarized as:

1) To design autonomous mobile robot that is:

- Able to localize itself in environment

- Able to understand and map environment using sensor outputs

- Able to move in workspace to perform specific task

- Capable of avoiding obstacles and to reach final goal point

- Capable to adapt to the changes in its environment

2) To improve navigation efficiency using:

- Localization, mapping and trajectory tracking.

- Real-time obstacle/collision avoidance algorithm developed using occupancy grid method.

3) To integrate localization, mapping, trajectory tracking and obstacle avoidance algorithms.

The thesis is organized as follows:

Chapter 1 is the general introduction to thesis introduction and objectives

Chapter 2 discuss regarding different kinds of wheeled mobile robots, holonomic and nonholonomic constraints and kinematic model development that applied to class of wheeled mobile robots, constraints applicable to car-like robots and motion control task for car-like robots. 
Chapter 3 deals with mobile robot localization. Localization is an important task to be performed in navigation. This chapter introduces Bayesian algorithm for mobile robot localization, kinds of Kalman filter algorithms with a step by step description. The measurement required for EKF is obtained from odometry is discussed in this chapter. Finally implementation of EKF for localization in this thesis work along with selection of noise covariance matrices is explained in detail.

Chapter 4 discusses another important aspect of navigation that is the mapping of environment. Different mapping methods have been introduced and description of occupancy grid generation method is discussed in detail. Final section is methodology adopted in this work for development of occupancy grids considering filtering of noise from laser range finder readings.

Chapter 5 deals with path tracking of car-like mobile robot. Procedure for Dynamic modeling of a car like robot is discussed in detail. Selection of parameters and design for steering controller in this thesis is explained.

Chapter 6 introduces obstacle avoidance design for car-like mobile robot. The algorithm for obstacle avoidance with various cases and condition is developed.

Chapter 7 describes integration of localization, mapping, trajectory tracking and obstacle avoidance algorithm. The simulation results are presented and discussed.

Chapter 8 concludes by summarizing the results of this work as they pertain to development of obstacle avoidance algorithm, mapping and path tracking control. The future work is also suggested.

Each chapter has literature review in its first section that relates to that particular chapter. 


\section{Chapter 2: Wheeled mobile robots}

This chapter deals with classification of wheeled mobile robots, constraints and kinematics and dynamic models for wheeled mobile robots (WMRs) used for control, guidance and navigation.

\subsection{Introduction}

WMRs are most widely used among the class of mobile robots due to their fast maneuvering, simple controllers, and energy saving characteristics. This long term interest has been mainly fueled by the myriad of practical applications that can be uniquely addressed by mobile robots due to their ability to work in large domains. WMRs are used in many applications which cover mining, planetary exploration, military tasks, monitoring nuclear activities, etc. All these applications require accurate sensing of environment, intelligent trajectory planning, collision avoidance and high precision control. Significant amount of research and development has been carried out over the past three decades in the area of WMRs. Of these, great deal of work has been carried out in development of different control strategies for motion tracking of WMRs ([2],[3],[4]) path/motion planning ([1],[5],[6],[7])and collision avoidance $([8],[9],[10])$. Control of WMRs has also been studied from several points of view, including set point stabilization, tracking, path following, etc.

Wheeled mobile robots constitute a class of mechanical systems referred to as nonholonomic systems. This nonholonomic nature of WMRs is related to the 
assumptions that the WMRs wheels bear pure rolling without slipping. As a consequence, these systems are characterized by kinematic constraints that are not integrable and therefore cannot be eliminated from model equations. A variety of models of this type of mobile robots have been developed and their dynamics have bee studied ([1], [11]). Due to nonlinear characteristics and amount of complexity involved, controller design for WMRs are usually based on kinematic models rather than dynamic models and are not solved by method of linear control. Analytical methods for controller design are well suited to kinematics based system; however, introducing dynamics into WMRs model is a complex task to perform analytically. Different examples for wheeled mobile robots are shown below:

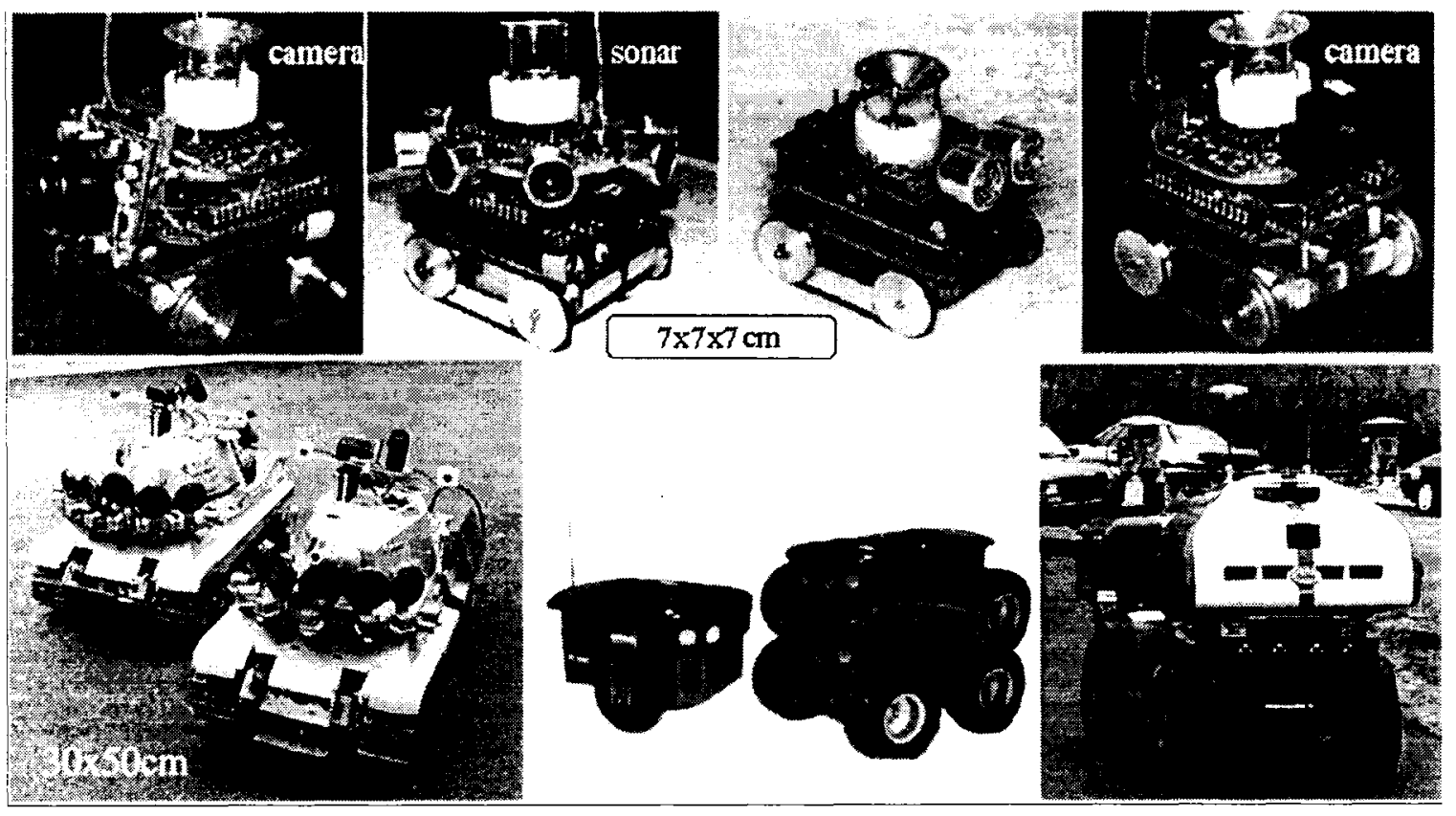

Figure 2.1: Examples of WMRs [12]

A mobile robot, whose configuration is defined by set of generalized co-ordinates in the space configuration, is characterized by constraint equations including geometric and 
kinematic constraints that involve the time derivatives of the system configuration variables. These equations are non integrable, arise when the system has less controls than the configuration. This unique feature leads to some challenging problems in the synthesis of feedback controllers. For instance, a car-like robot has only two controls, that is, linear and angular velocities as compared to three dimensional space configurations which are $(x, y, \theta)$, where $x$ and $y$ denotes the location along $\mathrm{X}$ and $\mathrm{Y}$ axes of horizontal plane and $\theta$ represents the orientation with respect to horizontal axis generally $\mathrm{X}$ axis. As a consequence, any path in the configuration space does not necessarily correspond to feasible path for the system. Car-like robot can move forward and backward and can make turns. However, it cannot move sideways and turns are limited by mechanical stops in the steering gear. Nevertheless, it can take any position and orientation in the plane. Hence the configuration reached by holonomic systems may not be reached by nonholonomic systems. As mentioned above, the constraints equations are expressed as equations relating the system configuration variables. If these equations are used to eliminate some parameters and reduce the dimension of the configuration space giving the form $a_{i}(q)=k_{i}$, where, $q$ is the vector of generalized co-ordinates, $k_{i}$ are some constants, and the motion is restricted to a level surface of $a_{i}$ they are called holonomic constraints. On the other hand, the equations involving velocity parameters of robot that do not reduce the dimensions of configuration space, but that of its velocity space are known as nonholonomic constraints. 


\subsection{Holonomic Constraints}

Consider that a configuration ' $\mathrm{q}$ ' is represented by list of parameters of minimal cardinality. A holonomic equality constraint is an equality relation among these parameters that can be solved for one of the parameters. Such equality relation reduces the dimension of actual configuration space of robot by one. For instance, consider a three dimensional body is allowed to translate freely, but is constrained to rotate around a fixed axis. If the orientation of the body is represented by three angles, then the constraints restricting the orientations can be expressed as two independent equations among these angles, hence reducing the dimension in configuration space to four from six possible dimensions of 3-dimensional workspace. The constraint equation defined over the configurations of a body is given as [5]:

$$
F(q, t)=F\left(q_{1}, q_{2}, \ldots, q_{m}, t\right)=0
$$

where, $\mathrm{F}$ is a smooth function with non-zero derivative.

Another example for holonomic constraint is articulated robots. The revolute joint in articulated robots constraints the motion in two dimensions; hence only four parameters are sufficient to define the configuration of robot rather than six parameters as in 3dimensional workspace.

Definition: [5]:

"A scalar constraint of the form $F(q, t)=0$, where $\mathrm{F}$ is a smooth function with non-zero derivative, is called a holonomic equality constraint". 


\subsection{Non holonomic constraints}

As discussed previously, a nonholonomic equality constraint is a non-integrable equation that consists of the configuration parameters along with its derivatives or velocity parameters. Such constraints do not reduce the dimension of space of configurations reachable by robot, but reduce the dimension of space of velocities (or motion) at any given configuration.

The example for nonholonomic mechanical systems is car-like robot.

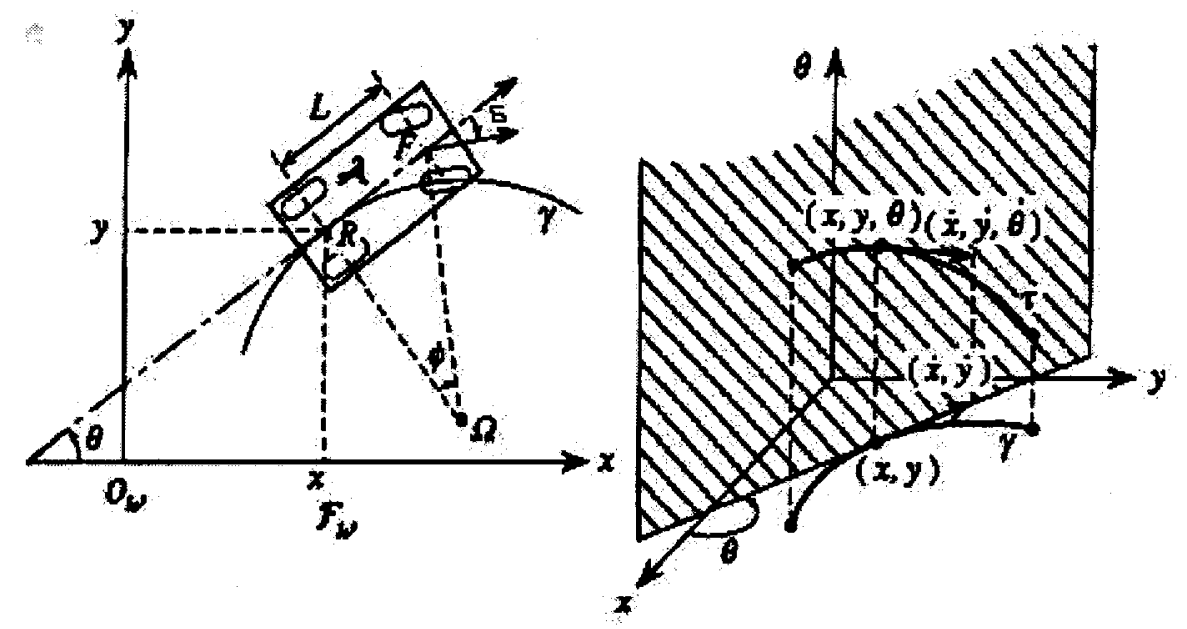

Figure 2.2: Car-like robot [5]

(a) A car-like robot moving in a plane

(b) Possible velocity vectors at each configuration.

As shown in figure above, the configuration is given as $(x, y, \theta)$, where $x$ and $y$ denotes the location of centre of mass of car-like robot along $\mathrm{X}$ and $\mathrm{Y}$ axes of horizontal plane and $\theta$ represents the orientation of that point with respect to horizontal axis normally $\mathrm{X}$ axis. At each instant, the velocity $(\dot{x}, \dot{y})$ acts along the main axis of car-like robot. Hence, 
the possible velocity vectors $(\dot{x}, \dot{y}, \dot{\theta})$ at each configuration span a plane. The angle $\delta$ between the main axis of car-like robot and the velocity of point at the centre of front axle is steering angle. When the robot moves, the centre of mass with configuration $(x, y, \theta)$ describes a curve $\gamma$ that must be tangent to the main axis of the car-like robot. Hence, its motion is constrained by:

$$
-\dot{x} \sin \theta+\dot{y} \cos \theta=0
$$

For car-like robot, let $\delta$ be the steering angle made by the velocity vector of $\mathrm{M}$, a midpoint of front axle with the main axis of robot, then the mechanical stops in the steering gear constrain $\delta$, such that,

$$
|\delta| \leq \delta_{\max }<\frac{\pi}{2}
$$

Here, we have limited the steering angle, $\delta$ to \pm 45 degrees (+ indicates left and indicates right).

As a consequence, the reference point, that is, centre of mass, must follow a curve whose curvature is upper-bounded by:

$$
\frac{1}{\rho_{\min }}=\frac{1}{L} \tan \theta
$$

Where, $\mathrm{L}=$ distance between the front and the rear axle or wheel base.

$$
\rho_{\min }=\text { minimum turning radius. }
$$

Since $\mathrm{L}=1.5 \mathrm{~m}$ in our case (figure 2.8 ), and $\delta_{\max }=45$ degrees, we have minimum

$$
\text { turning radius as: } \quad \Rightarrow \quad \frac{1}{\rho_{\min }}=\frac{1}{1.5} \tan 45
$$




$$
\begin{aligned}
& \Rightarrow \quad \frac{1}{\rho_{\min }}=0.67 \mathrm{~m}, \\
& \Rightarrow \quad \rho_{\min }=1.5 \mathrm{~m} .
\end{aligned}
$$

A car-like robot subjected to condition $|\delta| \leq \delta_{\max }<\frac{\pi}{2}$ is said to have limited steering angle or lower-bounded turning radius. If $v$ is the velocity of the reference point which is allowed to vary in some interval [ $\left.-v_{\max },+v_{\max }\right]$, and if $v>0$ and $\delta>0$, direction of motion is left forward, similarly if $v>0$ and $\delta<0$, direction of motion is right forward. Since in this work the robot is assumed to move only in forward direction, $v$ is always positive. The constraint $|\delta| \leq \delta_{\max }<\frac{\pi}{2}$ can be rewritten as:

$$
\begin{gathered}
|\dot{\theta}| \leq \frac{|v|}{\rho_{\min }} \text {, or } \\
\dot{x}^{2}+\dot{y}^{2}-\rho_{\text {min }}^{2} \dot{\theta}^{2} \geq 0
\end{gathered}
$$

Hence, at any configuration $\mathrm{q}$, it imposes that the velocity vector $(\dot{x}, \dot{y}, \dot{\theta})$ point into a two-sided cone of angle $2 \tan ^{-1}\left(1 / \rho_{\min }\right)$.

\section{Definition: [5]}

"A non-integrable scalar constraints of the form $G\left(q_{1}, q_{2}, \ldots ., q_{m}, \dot{q}_{1}, \dot{q}_{2}, \ldots, \dot{q}_{m}, t\right)=0$, Where G is a smooth function, is called a nonholonomic equality constraint." 


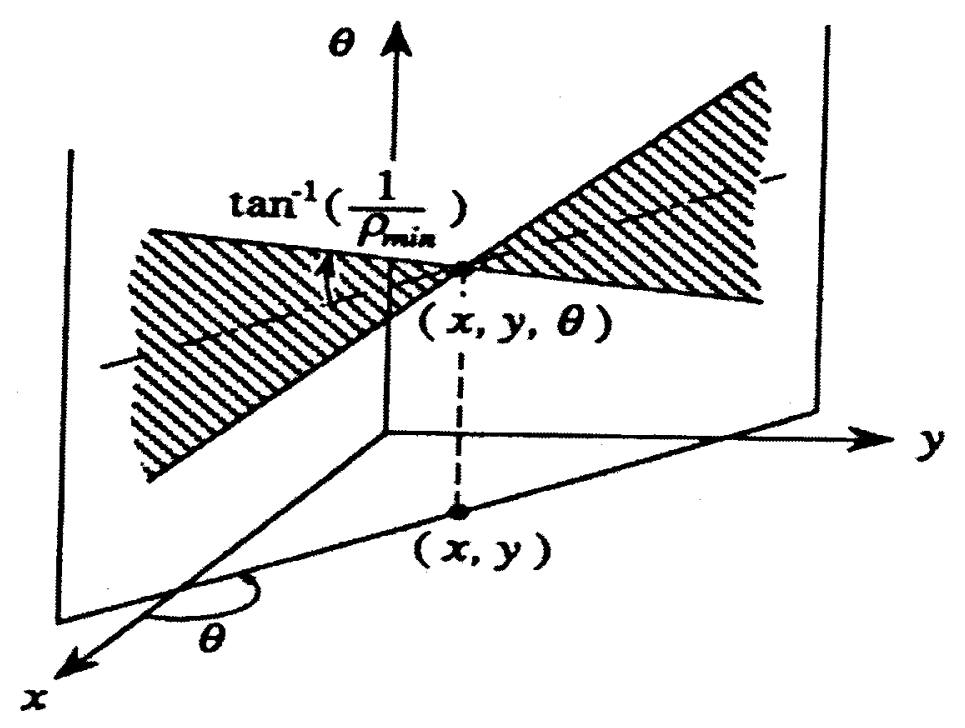

Figure 2.3: Velocity vector of car-like robot whose turning radius is bounded by $\rho_{\min }[5]$

\subsection{Motion control tasks for car-like robots}

There are two main control tasks for nonholonomic wheeled mobile robots. First is stabilizing to an equilibrium point, such as parking problem which is also known as point to point motion problem and second one is stabilizing to an equilibrium manifold such as trajectory tracking or path following problem. In first case the information regarding the final and initial configurations are stored in the robot memory. For instance, in car-like robot, the final as well as initial configurations are in the form of two Cartesian coordinates along horizontal plane, its orientation with respect to reference axis of global frame and steering angle with respect to frame of Vehicle (or local frame). 


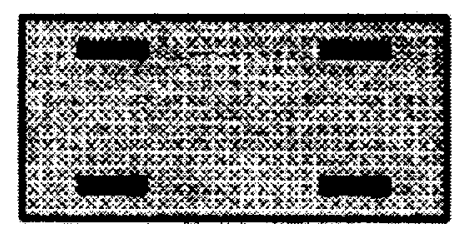

GOAL

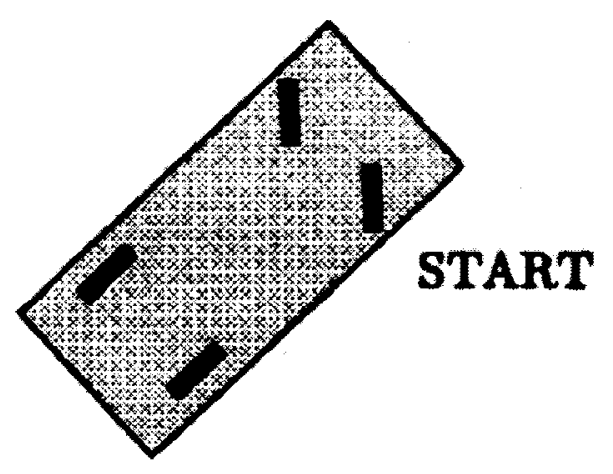

Figure 2.4: Point-to-point motion problem

The task of point stabilization is considered challenging because a nonholonomic system cannot be stabilized to an equilibrium point by a smooth state feedback [13], [14]. To overcome these difficulties, substantial efforts have been spent to develop sophisticated state-feedback-control laws, such as non smooth feedback laws [15], [16], time-varying feedback laws [17], [18], and middle (non smooth and time-varying) feedback laws [19], [19]. The second problem is the stabilization to an equilibrium manifold such as path following and trajectory tracking and is not subject to the difficulties as in the previous case. Since, the outputs that have the same dimension as the inputs can be defined, classical nonlinear control theories can be used to solve the output-tracking problem of nonholonomic systems. 


\section{Path following}

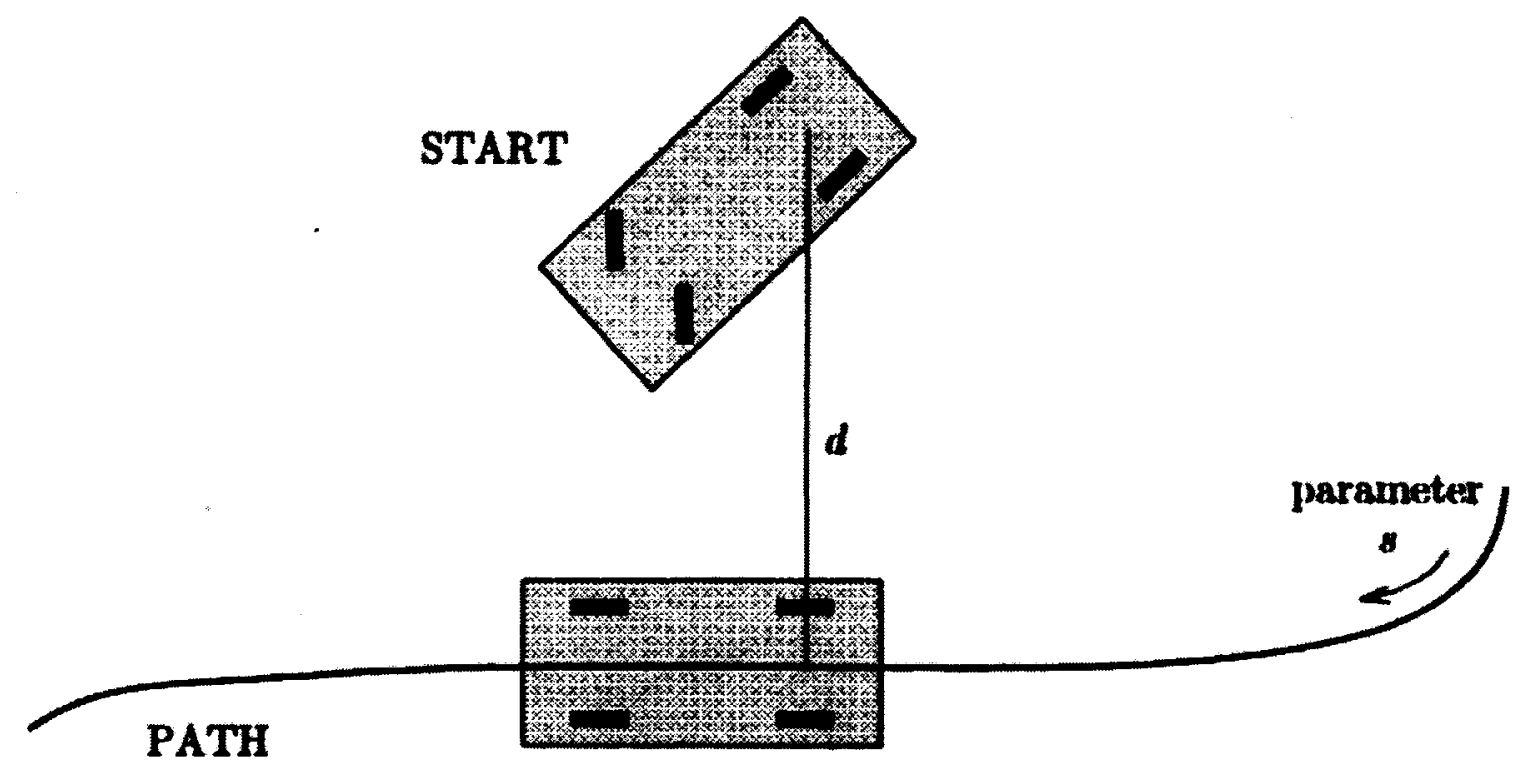

Figure 2.5: stabilization at equilibrium manifold: path following

Path following is the problem in which the robot must have to reach desired goal configuration from its given initial configuration (on or off the path). The robot planner is feed with initial configuration and final configuration. This information is usually provided in the parameterized form approximating the desired path motion in terms of path parameter that could be arc length or radius of curvature. For this task, time dependence is not necessary because one is concerned only with the geometric displacement between robot and path. With reference to this, the time evolution of path parameter is usually free, and the command inputs can be arbitrarily scaled with respect to time without changing the resultant robot path. Generally, one of the robot forward velocities is kept constant and using second one as control input. In this work the linear velocity is kept constant and the angular velocity of steering wheel (rate of change of 
steering angle) is used as control input to control the direction of car-like robot so as to move on desired path or during collision avoidance when object on the desired path is detected. The path following problem is thus rephrased as the stabilization to zero of a suitable scalar path error function.

\section{Trajectory tracking}

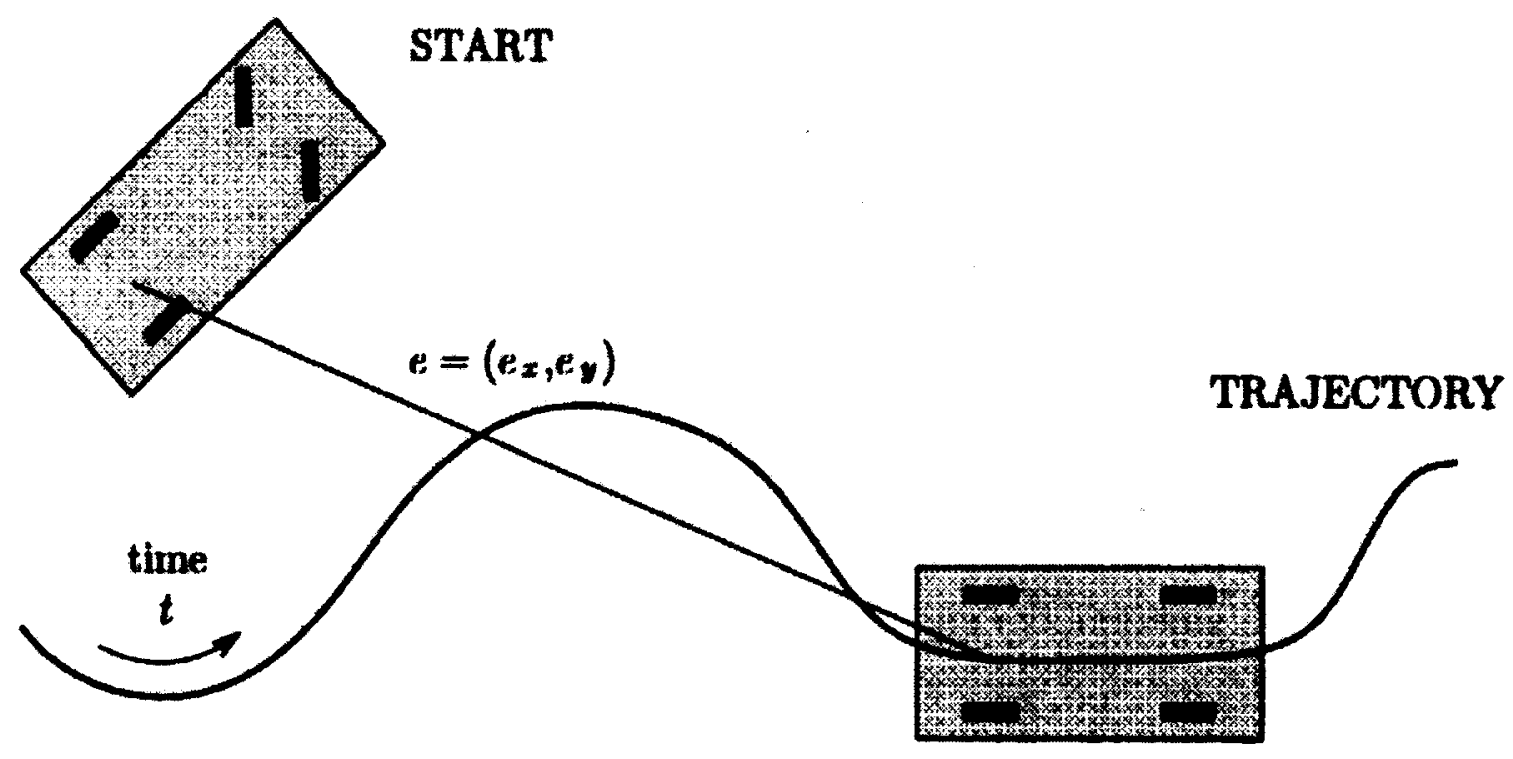

Figure 2.6: Stabilization at equilibrium manifold: Trajectory tracking

In trajectory tracking problem the robot must reach and follow a trajectory in the Cartesian space with associated time starting from given initial to final configuration (on or off the trajectory). Unlike path following, the robot must follow the desired Cartesian path with a specified timing law. Although the trajectory can be split into several parameterized geometric paths and a timing law for the parameters, such separation is not strictly necessary. Sometimes, it is simpler to specify the workspace trajectory as the 
desired time evolution for the position of some specific point of robot. Trajectory tracking problem deals with the stabilization to zero of the two dimensional Cartesian error using control inputs. In many literatures, for example [4], for the control of car-like robot the errors considered are distance, orientation and steering angle. For car-like robot here considered in this work, the steering angle error, lateral distance error and the orientation error at specific point on trajectory are stabilized to zero for the control of all four configuration variables. For path following, a local solution means that the controller works properly provided we start sufficient close to the path while in case of trajectory tracking, closeness should be evaluated with respect to the current position the robot.

\subsection{Modeling and Analysis of Car-like robot}

This section discusses about kinematic model for different kinds of wheeled mobile robots such as unicycle, car-like robots are considered. The presence on nonholonomic constraints considering wheeled mobile robots are assumed to have motion with pure rolling without slipping between the wheels and ground is main features for kinematic model of wheeled mobile robot. 


\section{1) Unicycle}

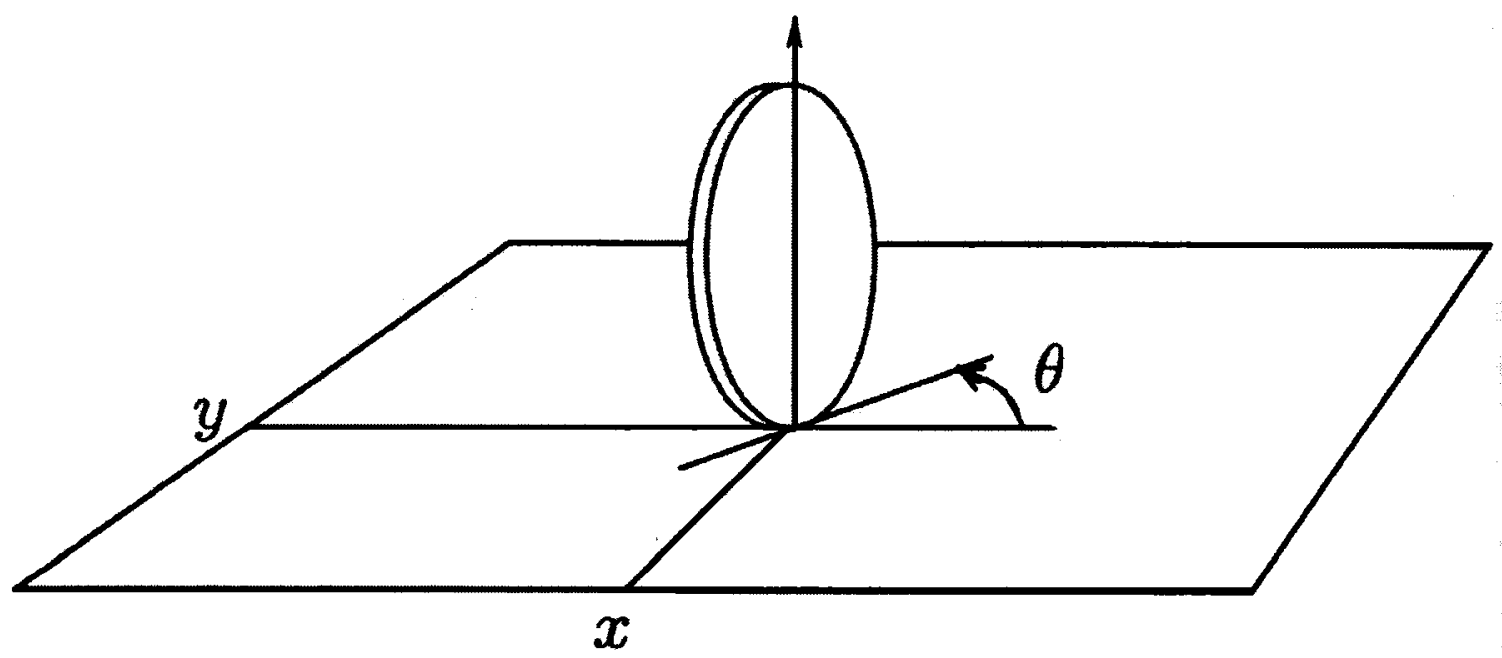

Figure 2.7: Unicycle

Unicycle is considered as a robot with a single wheel. Consider the wheels rolls on the plane with its body vertical. The configuration $\mathrm{q}$ with three generalized co-ordinates is given as:

$$
q=(x, y, \theta)
$$

Where, $x, y$ are the co-ordinates of the point of contact with the horizontal plane and $\theta$ is the orientation of wheel with respect to reference axis $\mathrm{X}$. The system generalized velocities $\dot{q}$ cannot assume independent values and must satisfy the constraint of the form:

$$
-\dot{x} \sin \theta+\dot{y} \cos \theta=0
$$

Hence the above equation can be written as the form of Pfaffian Constraint $C(q) \dot{q}=0$ :

$$
\left(\begin{array}{lll}
\sin \theta & -\cos \theta & 0
\end{array}\right)\left(\begin{array}{c}
\dot{x} \\
\dot{y} \\
\dot{\theta}
\end{array}\right)
$$


The constraint matrix is:

$$
\begin{aligned}
\mathbf{C}(q) & =\left(\begin{array}{ll}
\cos \theta & 0 \\
\sin \theta & 0 \\
0 & 1
\end{array}\right) \\
& =\left(\begin{array}{ll}
c_{1} & c_{2}
\end{array}\right)
\end{aligned}
$$

Hence, the kinematic constraint can be expressed as:

$$
\begin{aligned}
\dot{q} & =\mathbf{C}(q) u \\
& =\left(\begin{array}{l}
\cos \theta \\
\sin \theta \\
0
\end{array}\right) u_{1}+\left(\begin{array}{l}
0 \\
0 \\
1
\end{array}\right) u_{2} \\
& =c_{1}(q) u_{1}+c_{2}(q) u_{2}
\end{aligned}
$$

Where, $u_{1}$ is linear velocity input of the wheel and $u_{2}$ is steering velocity input.

This equation is kinematic equation for unicycle. All admissible velocities are contained in the null space of the constraint matrix $\mathbf{C}(q)$.

\section{2) Car-like wheeled mobile robot}

It may be considered for simplicity as a bicycle model where it is assumed that the two wheels of front and rear axle collapse into a single wheel located at the midpoint of the axle. The front wheel can be steered while the rear wheel is not allowed to steer with fixed orientation. The configuration $\mathrm{q}$ with four generalized co-ordinates is:

$$
q=(x, y, \theta, \delta)
$$


Where, $x, y$ are the co-ordinates of centre of mass of the vehicle and acts as a reference point, $\theta$ is the orientation of that point with respect to reference axis $\mathrm{X}$ and $\delta$ is the steering angle.

The car-like wheeled mobile robot is as shown in figure below:

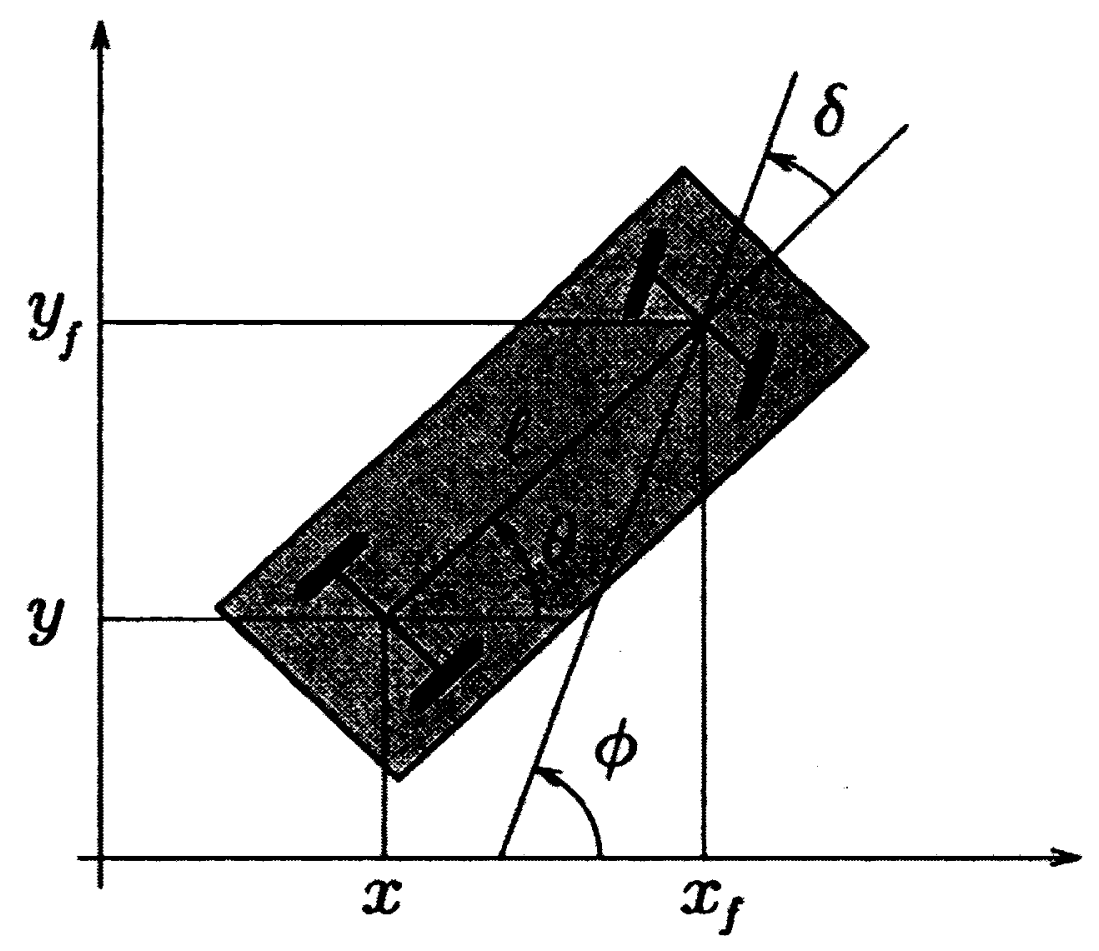

Figure 2.8: Car-like wheeled mobile robot.

The nonholonomic constraints acting on first and second wheels are given respectively as:

$$
\begin{gathered}
\dot{x}_{f} \sin (\theta+\delta)-\dot{y}_{f} \cos (\theta+\delta)=0 \\
\dot{x} \sin \theta-\dot{y} \cos \theta=0
\end{gathered}
$$

Here, $x_{f}$ and $y_{f}$ are the coordinates of front wheel and are given as:

$$
x_{f}=x+l \cos \theta
$$




$$
y_{f}=y+l \sin \theta
$$

$l=$ distance between front and rear wheel or wheel base.

Now, substituting the value of $x_{f}$ and $y_{f}$ in first equation, we get:

$$
\dot{x} \sin (\theta+\delta)-\dot{y} \cos (\theta+\delta)-\dot{\theta} l \cos \delta=0
$$

Hence, the Pfaffian constraint matrix becomes:

$$
\mathbf{C}(q)=\left(\begin{array}{cccc}
\sin (\theta+\delta) & -\cos (\theta+\delta) & -l \cos \delta & 0 \\
\sin \theta & -\cos \theta & 0 & 0
\end{array}\right)
$$

Now, there are two kinematic models for car-like robots that are, one with front wheel drive and another with rear wheel drive and are as shown below. In this work, we have considered the model having rear weal drive for our study.

Kinematic model for a car-like robot having rear wheel drive:

$$
\left(\begin{array}{l}
\dot{x} \\
\dot{y} \\
\dot{\theta} \\
\dot{\delta}
\end{array}\right)=\left(\begin{array}{l}
\cos \theta \\
\sin \theta \\
\tan \delta / l \\
0
\end{array}\right) u_{1}+\left(\begin{array}{l}
0 \\
0 \\
0 \\
1
\end{array}\right) u_{2},
$$

The Pfaffian constraint matrix for this case will be:

$$
\mathbf{C}(q)=\left(\begin{array}{cc}
\cos \theta & 0 \\
\sin \theta & 0 \\
\tan \delta / l & 0 \\
0 & 1
\end{array}\right)
$$

From this we can observe that the model possesses singularity when $\delta= \pm \frac{\pi}{2}$. Because of this the car gets jammed when the front wheel is normal to the longitudinal axis of the robot. Because of this reason we have limited the maximum value of $\delta$ at \pm 45 degrees. 
Kinematic model for front wheel drive case is given as:

$$
\left(\begin{array}{l}
\dot{x} \\
\dot{y} \\
\dot{\theta} \\
\dot{\delta}
\end{array}\right)=\left(\begin{array}{l}
\cos \theta \cos \delta \\
\sin \theta \cos \delta \\
\sin \delta / l \\
0
\end{array}\right) u_{1}+\left(\begin{array}{l}
0 \\
0 \\
0 \\
1
\end{array}\right) u_{2}
$$

$u_{1}$ and $u_{2}$ are linear velocity of non steering wheel and steering velocity of steering wheel respectively. The Pfaffian constraint matrix for this case will be:

$$
\mathbf{C}(q)=\left(\begin{array}{cc}
\cos \theta \cos \delta & 0 \\
\sin \theta \cos \delta & 0 \\
\sin \delta / l & 0 \\
0 & 1
\end{array}\right)
$$

These kinematic models can also be converted to simpler forms using change of variable method but is not discussed here. 


\section{Chapter 3: Mobile robot localization}

\subsection{Introduction and literature review for mobile robot localization}

Localization is a major component providing positions, directions and travel information to driver. To travel mobile robot from one location to another, it has to know its position and orientation at any given time. Many approaches for mobile robot localization have been proposed. The most commonly used method includes dead-reckoning techniques, navigation using active beacons, etc. Mobile robot localization is one of the most basic and most important tasks to be performed in world of robotics. It plays a pivotal role in various successful mobile robot navigation systems. It has been referred to as "the most fundamental problem to providing a mobile robot with autonomous capabilities" [10]. The states are the position and orientation of the robot in given map of environment, while the measurements are used to update the states of robot at definite periodic interval obtained from the sensors mounted at definite location with respect to frame of robot. This estimation is required because of various factors, one of these; the most prominent is the presence of noise in sensor output that leads to increase error in location of robot which continuously piles up with prolonged period of operation. For example, encoders mounted on wheel of robot though have very less error by itself but most error is built up because of presence of slippage between the wheels and road or path on which robot is moving. Presence of loose soil present on mars or in mine like environment also leads to such errors. There are large volumes of the environment that are not sensed by sensors. For example, a CMOS based camera and a CCD based camera will provide a different 
color readings even when used in the same environment due to different technology underlying the camera. The environment might itself contribute natural noise processes to affect the image taken by camera, such as fog [17]. The initial pose is known and incremental errors are compensated in a robot's odometry. Algorithms for position tracking assume the nature and size of error. More challenging is the global localization problem. The global localization problem is more difficult, since error in robots estimated cannot be assumed to be small. The more difficult problem is "kidnapped robot problem". The kidnapped robot problem is often used to test the robot's ability to recover from localization failures. Bayesian state estimation is most popular method used for mobile robot localization.

\subsection{Bayesian algorithm for mobile robot localization}

Many dynamic problems require estimation of state of a system that changes over time using a sequence of noisy measurements made on system. The state space approach is considered in order to model the system.

$$
\begin{aligned}
\mathbf{x}_{k} & =\mathbf{A x}_{k-1}+\mathbf{B} \mathbf{w}_{k-1} \text { For linear system and } \\
\mathbf{y}_{k} & =\mathbf{C x}_{k}+\mathbf{D} \mathbf{v}_{k} \\
\mathbf{x}_{k} & =f_{k}\left(\mathbf{x}_{k-1}, \mathbf{w}_{k-1}\right) \text { For non-linear system } \\
\mathbf{y}_{k} & =h_{k}\left(\mathbf{x}_{k}, \mathbf{v}_{k}\right)
\end{aligned}
$$

where, $\mathbf{B}=\mathbf{D}=1, \mathbf{w}=$ system noise, $\mathbf{v}=$ observation noise 
It consists of evolution model (or prediction) and observation (or measurement) model. Evolution model describes the transition of system form one state to another state in presence of system noise. Generally, the system noise is modeled as Gaussian noise with zero mean and known covariance. State vector contain the parameters of the system that are required to estimate. For example, in tracking problem, this information could be related to the kinematics characteristics of the target. Alternatively, in an econometrics problem, it could be related to monetary flow, interest rates, inflation, etc. Measurement model consists of measurement vector with measurement noise and the dimension is generally lower than state vector.

In this work, we assume that models are available in probabilistic form. The probabilistic state space formulation and the requirement for the updating are ideally suited for Bayesian approach.

Bayesian filters are recursive filters where states are predicted using system dynamic equation and are updated as new information is available in the form of measurement. Hence it needs to construct posterior probability density function (pdf) of the states given all measurements in presence of noise. A recursive filtering approach means that received data can be processed sequentially rather than as a batch so that it is not necessary to store the complete data set or to reprocess existing data if a new measurement becomes available. For linear as well as nonlinear systems, Kalman filters are best suited but they assume Gaussian dynamics. In this thesis, EKF is used to estimate robot pose taking into account encoder error covariance. Consider nonlinear system model as shown in (3.2). It 
is assumed that initial pdf $p\left(\mathbf{x}_{0}\right)$ of the state vector and prori $p d f \quad p\left(\mathbf{x}_{k-1}, \mathbf{y}_{1: k-1}\right)$ are known. The posterior pdf $p\left(\mathbf{x}_{k} \mid \mathbf{y}_{1: k}\right)$ may be obtained in two stages:

Prediction: $p\left(\mathbf{x}_{k}, \mathbf{y}_{1: k-1}\right)=\int p\left(\mathbf{x}_{k}, \mathbf{x}_{k-1}\right) p\left(\mathbf{x}_{k-1}, \mathbf{y}_{1: k-1}\right) d \mathbf{x}_{k-1}$

Update: $\quad p\left(\mathbf{x}_{k}, \mathbf{y}_{1: k}\right)=\frac{p\left(\mathbf{y}_{k} \mid \mathbf{x}_{k}\right) p\left(\mathbf{x}_{k}, \mathbf{y}_{1: k-1}\right)}{p\left(\mathbf{y}_{k} \mid \mathbf{y}_{1: k-1}\right)}$

Where,

$$
p\left(\mathbf{y}_{k} \mid \mathbf{y}_{1: k-1}\right)=\int p\left(\mathbf{y}_{k} \mid \mathbf{x}_{k}\right) p\left(\mathbf{x}_{k}, \mathbf{y}_{1: k-1}\right) d \mathbf{x}_{k}
$$

is normalizing constant.

where, $p\left(\mathbf{x}_{k}, \mathbf{x}_{k-1}\right)=$ system evolution model

\subsection{Kalman filters}

"Kalman filter is an effective recursive filter which estimates the state of a dynamic system from a series of incomplete and noisy measurements" [18]. Kalman filter is named after its inventor Rudolf E. Kalman who developed the algorithm in early 60's. Varieties of Kalman filters have been developed from original algorithm now known as simple Kalman filter. Its is based on linear dynamical system operating on discrete time domain taking into consideration measurement and system noise while estimating the states of system. It assumes the variables have Gaussian statistics. Since many natural processes are Gaussian distributed and also Non-Gaussian, independent process approaches to Gaussian statistics due to Central Limit theorem. Hence Kalman filter is considered as optimal filter provided the assumptions are valid. Kalman filters are also used for nonlinear system by carrying out local linearization of nonlinear process through 
partial derivatives which is known as EKF and will be discussed in following section. During estimation of states, Kalman filter keeps tracks of mean and covariance at every step hence does not lose any information during its execution. Being recursive in nature, no need to store data and thus reduces computational time and memory usage to large extent.

\subsubsection{Discrete Kalman Filter}

Here we shall see the basic generic algorithm for discrete Kalman filter for estimation of states for the system or random process. The discrete time state space equations are written of the form [18]:

$$
\begin{aligned}
& \mathbf{x}_{k+1}=\varphi_{k} \mathbf{x}_{k}+\mathbf{w}_{k} \\
& \mathbf{z}_{k}=\mathbf{H}_{k} \mathbf{x}_{k}+\mathbf{v}_{k}
\end{aligned}
$$

Where,

$$
\begin{aligned}
\mathbf{x}_{k}= & \text { process state vector at time } t_{k} \text { of order }(n \times 1) \\
\boldsymbol{\varphi}_{k}= & \text { state transition matrix relating } \mathbf{x}_{k} \text { with } \mathbf{x}_{k+1} \text { of order }(n \times n) \\
\mathbf{w}_{k}=\text { system noise sequence vector assumed to be white of order }(n \times 1) & \mathbf{z}_{k}=\text { process measurement vector at time } t_{k} \text { of order }(m \times 1) \\
\mathbf{H}_{k}=\text { matrix represents the relation between states and measurements at time } & \\
& { }_{t} \text { of order }(m \times n) \\
\mathbf{v}_{k}= & \text { measurement noise sequence vector assumed to be white of order }(m \times 1)
\end{aligned}
$$

The covariance matrices for $\mathbf{w}_{k}$ and $\mathbf{v}_{k}$ are given as:

$$
\begin{aligned}
E\left\{\mathbf{w}_{\mathbf{k}} \cdot \mathbf{w}_{\mathbf{i}}^{\mathbf{T}}\right\} & =\mathbf{Q}_{k}, & & \text { for } i=k \\
& =0, & & \text { for } i \neq k
\end{aligned}
$$




$$
\begin{aligned}
& E\left\{\mathbf{v}_{\mathbf{k}} \cdot \mathbf{v}_{\mathbf{i}}^{\mathbf{T}}\right\}=\mathbf{R}_{k}, \quad \text { for } i=k \\
& =0, \quad \text { for } i \neq k \\
& E\left\{\mathbf{w}_{k} \cdot \mathbf{v}_{i}^{T}\right\}=0, \quad \text { for all values of } k \text { and } i
\end{aligned}
$$

In order to derive estimates at time $t_{k}$, it is necessary to have prior knowledge of the states prior to time $t_{k}$ which is denoted as $\hat{\mathbf{x}}_{\mathbf{k}}{ }^{-}$. The error associated with it is given as $\mathbf{e}_{k}=\mathbf{x}_{k}-\hat{\mathbf{x}}_{k}{ }^{-}$and error covariance matrix:

$$
\mathbf{P}_{\mathbf{k}}{ }^{-}=\left\{\mathbf{e}_{k}{ }^{-} \cdot \mathbf{e}_{k}^{-T}\right\}=E\left\{\left(\mathbf{x}_{k}-\hat{\mathbf{x}}_{k}{ }^{-}\right) \cdot\left(\mathbf{x}_{k}-\hat{\mathbf{x}}_{k}{ }^{-}\right) T\right\}
$$

With this assumption we predict the states at time $\mathrm{t}$ as $\hat{\mathbf{x}}_{k}{ }^{-}$in prediction stage using system model as given by equation (3.6) and as soon as we receive the measurements from sensors $\mathbf{z}_{k}$, the prediction is corrected to improve the priori estimate $\hat{\mathbf{x}}_{k}{ }^{-}$by using the update equation which is given as:

$$
\hat{\mathbf{x}}_{k}=\mathbf{x}_{k}^{-}+\mathbf{K}_{k}\left(\mathbf{z}_{k}-\mathbf{H}_{k} \mathbf{x}_{k}^{-}\right)
$$

Where, $\mathbf{K}_{k}$ is the Kalman gain or blending factor and its derivation is derived as below:

First, consider the error covariance matrix as

$$
\mathbf{P}_{k}=\left\{\mathbf{e}_{k} \cdot \mathbf{e}_{k}^{T}\right\}=E\left\{\left(\mathbf{x}_{k}-\hat{\mathbf{x}}_{k}\right) \cdot\left(\mathbf{x}_{k}-\hat{\mathbf{x}}_{k}\right)^{T}\right\}
$$


Now, substituting the equations (3.11) into (3.12), we get,

$$
\mathbf{P}_{k}=E\left\{\begin{array}{l}
{\left[\left(\mathbf{x}_{k}-\hat{\mathbf{x}}_{k}{ }^{-}\right)-\mathbf{K}_{k}\left(\mathbf{H}_{k} \mathbf{x}_{k}+\mathbf{v}_{k}-\mathbf{H}_{k} \hat{\mathbf{x}}_{k}-\right] \times\right.} \\
{\left[\left(\mathbf{x}_{k}-\hat{\mathbf{x}}_{k}{ }^{-}\right)-\mathbf{K}_{k}\left(\mathbf{H}_{k} \mathbf{x}_{k}+\mathbf{v}_{k}-\mathbf{H}_{k} \hat{\mathbf{x}}_{k}-\right]^{T}\right.}
\end{array}\right\}
$$

Since $\left(\mathbf{x}_{k}-\hat{\mathbf{x}}_{k}^{-}\right)$is uncorrelated with measurement noise $\mathbf{v}_{k}$, we have,

$$
\mathbf{P}_{k}=\left(\mathbf{I}-\mathbf{K}_{k} \mathbf{H}_{k}\right) \mathbf{P}_{k}^{-}\left(\mathbf{I}-\mathbf{K}_{k} \mathbf{H}_{k}\right)^{T}+\left(\mathbf{K}_{k} \mathbf{R}_{k} \mathbf{R}_{k}^{T}\right)
$$

Finding the value of Kalman gain, that minimizes mean-square estimation error, requires minimization of error covariance matrix because the term it contains represents the estimation error variances for the elements of the state vector being estimated. Hence,

$$
\mathbf{K}_{k}=\mathbf{P}_{k}-\mathbf{H}_{k}^{T}\left(\mathbf{H}_{k} \mathbf{P}_{k}^{-} \mathbf{H}_{k}^{T}+\mathbf{R}_{k}\right)^{-1}
$$

And the updated covariance matrix associated with the optimal estimate is given as:

$$
\mathbf{P}_{k}=\left(\mathbf{I}-\mathbf{K}_{k} \mathbf{H}_{k}\right) \mathbf{P}_{k}^{-}
$$

Whose derivation is given in [18], finally the priori estimates for the next step is calculated as $\hat{\mathbf{x}}_{k+1}^{-}=\varphi_{k} \hat{\mathbf{x}}_{k}$

And priori error covariance matrix is given as $\mathbf{P}_{k+1}^{-}=\boldsymbol{\varphi}_{k} \mathbf{P}_{k} \boldsymbol{\varphi}_{k}^{T}+\mathbf{Q}_{k}$ 
All equations given above are calculated recursively to generate updated states at every time step. This summarizes what is now known as Kalman filter. The following diagram shows the flow of steps for discrete Kalman filter.

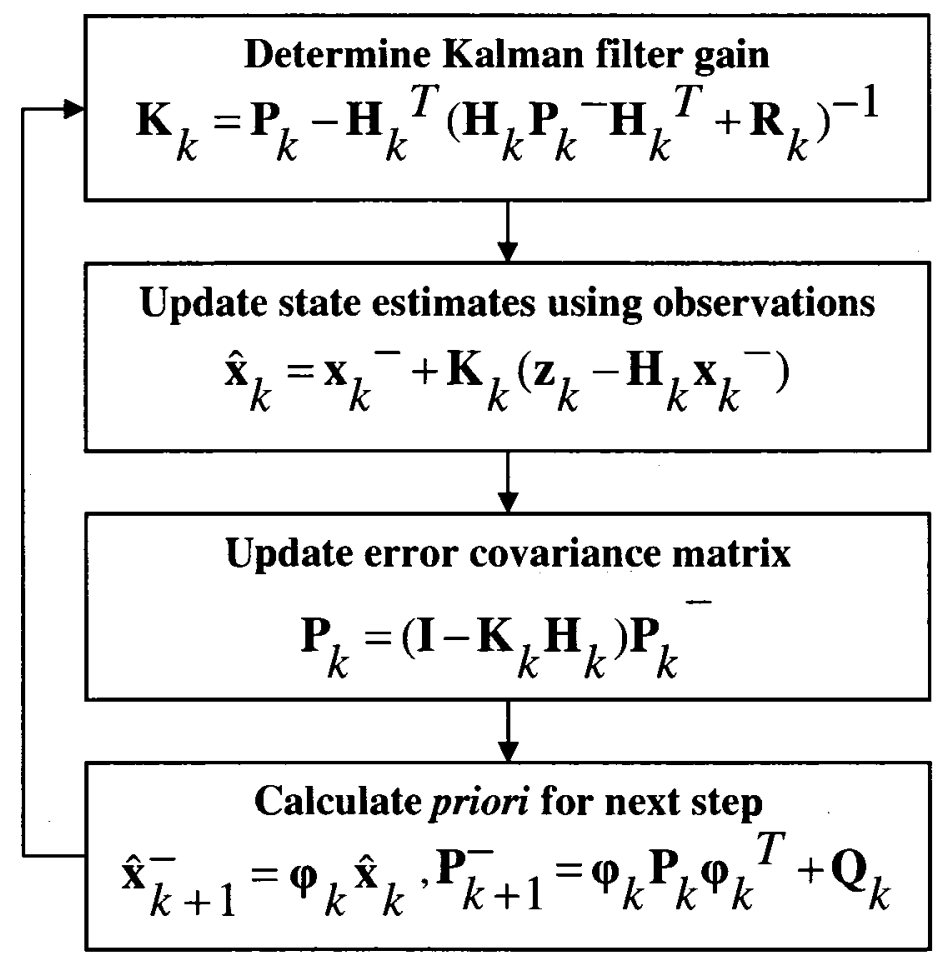

Figure 3.1: Steps for discrete Kalman filter

\subsubsection{Extended Kalman filter}

In previous section we discussed about discrete Kalman filter which assumes the system or process model in linear form but almost all the system or random process that we see around us are nonlinear so it is necessary to introduce the advanced version of basic Kalman filter that can be applied to expectation of states for nonlinear systems. In this 
case, local linearization of nonlinear system is carried out using Jacobian matrices. There are two ways to carry out linearization. One is to linearize about some nominal trajectory in state space that does not depend on the measurement data and is known as linearized Kalman filter. Another method is to linearize about the updated trajectory that is continuously being updated using measurements at each steps and is known as Extended Kalman filter. Consider the process to be estimated may be written in the form:

$$
\begin{aligned}
& \mathbf{x}_{k}=f_{k}\left(\mathbf{x}_{k-1}, \mathbf{w}_{k-1}\right)+\mathbf{u}(t) \\
& \mathbf{y}_{k}=h_{k}\left(\mathbf{x}_{k}, \mathbf{v}_{k}\right)+\mathbf{v}(t)
\end{aligned}
$$

Here we see that the term $\mathbf{u}$ and $\mathbf{v}$ are assumed to be separate and are not included in the terms $f$ and $h$. The functions $f$ and $h$ are approximated using Taylor's series expansions as shown in [18] and are shown below:

$$
\begin{aligned}
& \frac{\partial f}{\partial x}=\left[\begin{array}{l}
\frac{\partial f_{1}}{\partial x_{1}} \frac{\partial f_{1}}{\partial x_{2}} \ldots \\
\frac{\partial f_{2}}{\partial x_{1}} \frac{\partial f_{2}}{\partial x_{2}} \ldots \\
.
\end{array}\right] \\
& \frac{\partial h}{\partial x}=\left[\begin{array}{l}
\frac{\partial h_{1}}{\partial x_{1}} \frac{\partial h_{1}}{\partial x_{2}} \ldots \\
\frac{\partial h_{2}}{\partial x_{1}} \frac{\partial h_{2}}{\partial x_{2}} \ldots \\
.
\end{array}\right]
\end{aligned}
$$


These terms are evaluated along the trajectory that has been updated with the filter's estimates; which in turn depends on the measurements. Rest all other equations as described in the previous sections remains the same except $\mathbf{F}$ and $\mathbf{H}$ matrices are updated at every time step as soon as the measurements are available. The example for EKF is given in [18].

\subsection{Dead reckoning for localization}

The most common dead reckoning technique used for the ground vehicle is odometry. Odometry or optical shaft encoder is most widely used navigation methods for mobile robot positioning. It is inexpensive and provided very good short-term accuracy. The fundamental idea of odometry is the integration of incremental motion information over time. It is mounted on vehicle's driving wheels shaft and register angular movements of the wheel. As the duration of operation increases, it leads to accumulation of large roundoff errors also when there is no presence of slip between wheels and the roads. In case of indoor mobile robots there exists a small or negligible error due to slip when can be estimated by experiments and then is later compensated from the odometry readings so as to obtain error free location of robots. But, in case like, planetary rover or a vehicle moving in mines or fields where there is relatively high amount of slip, other navigational methods are used to enhance the performance of odometry by correcting it over specific period of time. Irregular changes in wheel and ground interaction make it difficult to compensate for the slippage. Incremental odometry using optical encoders on the wheels of the same axle gives both vehicle's translation and rotation information. But even in 
good conditions, with negligible slippage, the accuracy of measured rotational vehicle speed is very poor, in contrast to translation speed measurement.

Hence in our case an encoder is located at steering wheel shaft that measures the steering angle, which is later used to determine the orientation and hence position. The noise is considered as Gaussian and EKF is used to estimate the location and orientation of vehicle at every time step and is finally used to guard the vehicle on designed trajectory. The implementation of EKF with different noise covariance matrices is discussed in detail in later section. Various methods for establishing error covariance matrices are discussed by several authors such as [20], [21], [22]. A typical differential drive mobile robot is as shown in figure below. The dead reckoning is performed by using simple geometric equations to compute the momentary position of vehicle relative to a known starting position.

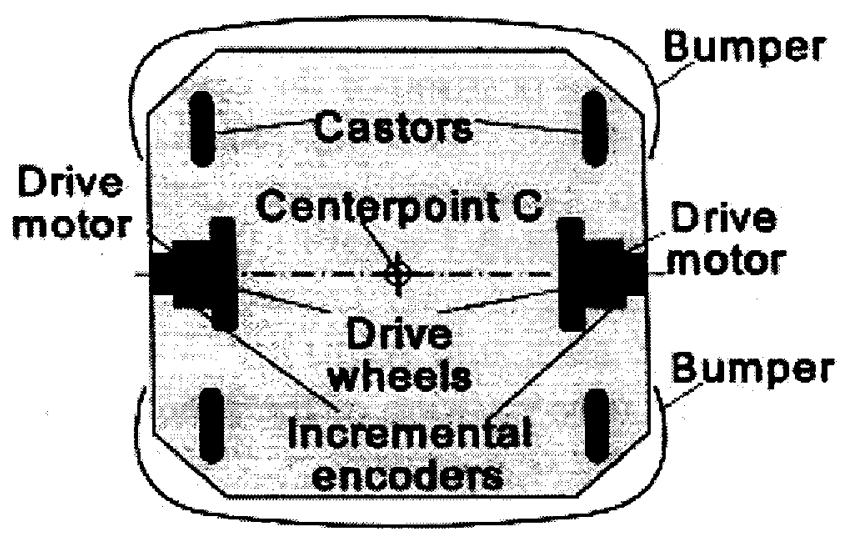

Figure 3.2: Typical differential-drive mobile robot [23] 
Suppose, for a particular sampling time interval, the number of samples generated by encoders is $M_{1}$ and $M_{2}$ respectively for left and right vehicle. The conversion factor ' $C$ ' is given as:

$$
C=\frac{\pi D}{n C e}
$$

Where, $\mathrm{C}=$ Conversion factor, $\mathrm{D}=$ Diameter of wheel, $\mathrm{Ce}=$ Encoder resolution in pulses/revolution, $\mathbf{n}=$ gear reduction ratio. The incremental distance traveled by each wheel during single sampling time period from figure as shown below is given as:

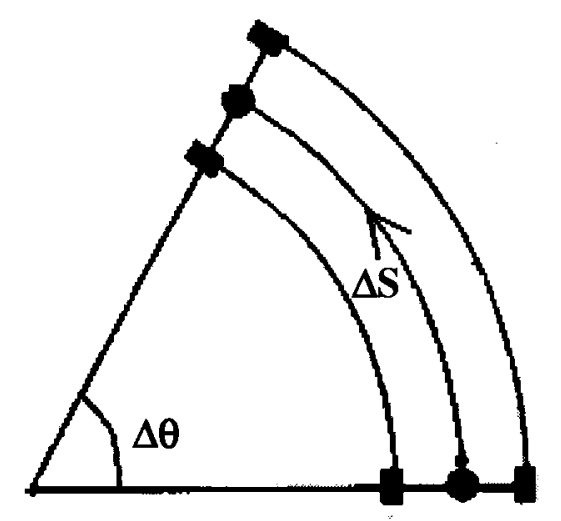

Figure 3.3: Distance traveled on curved path

$$
\Delta S_{r}=(L+R) \Delta \theta, \quad \Delta S_{l}=R \Delta \theta
$$

The incremental displacement of the robots centre point which is denoted as, $\Delta S$, is given by:

$$
\Delta S=\frac{\left(\Delta S_{r}+\Delta S_{l}\right)}{2}
$$

There are basically two types of errors, systematic and non-systematic errors. Systematic errors are mainly due to hardware of the system and the inaccuracies in the vehicle 
kinematics itself, for instance, unequal wheel diameters, average of actual wheel diameters differ from nominal wheel diameter, difference between actual and nominal wheel base, wheel misalignment, finite encoder resolution and sampling rates, whereas non-systematic errors are due to environmental or surrounding factors and are unpredictable, for instance, uneven floors, unexpected object on floors, slippage due to over acceleration, skidding, external and internal forces and non-point wheel contact with the floor. Systematic errors can be calibrated using experimental results and accumulate constantly. In indoor environments, systematic errors are more significant than nonsystematic errors and vice-versa. The accumulation of error with increase in period of operation is shown in figure below:

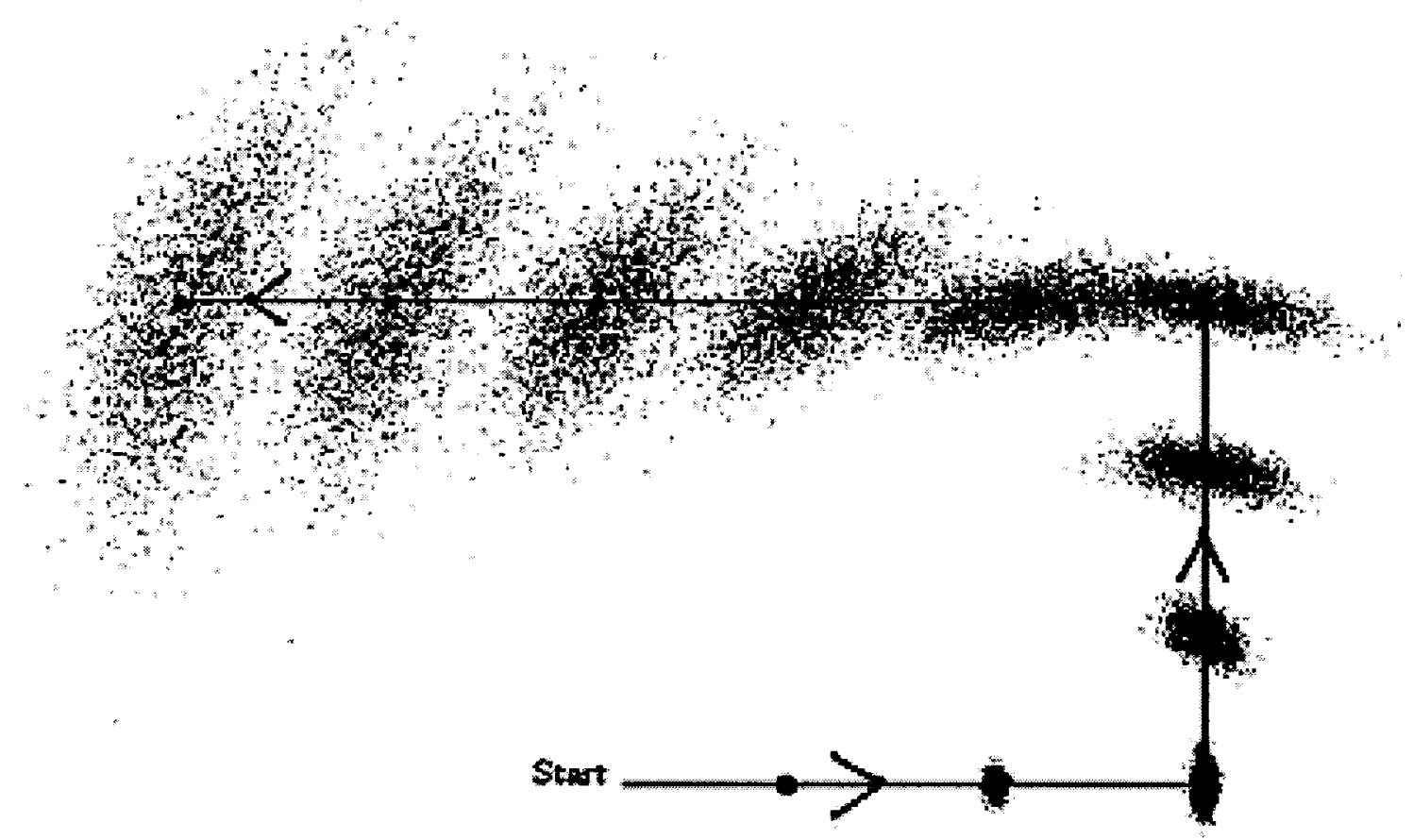

Figure 3.4: Accumulation of odometry errors with distance traveled. 


\subsection{Implementation of mobile robot localization}

From previous sections we can see that how important is localization problem in path planning and we also saw the recursive equations of EKF so as to obtain updated stated considering sensor information. In this section, the method adopted for mobile robot localization using odometry measurements in this work is discussed. Moreover, since the kinematic equations are nonlinear, EKF is used by linearization of nonlinear system about the updated states. Consider the kinematics equations for car-like mobile robot, will be discussed in chapter 5 , as:

$$
\dot{x}=v_{u} \cos \theta ; \dot{y}=v_{u} \sin \theta ; \dot{\theta}=\omega=\left(v_{u} / L\right) \times \tan \delta
$$

Linearized equations of these nonlinear kinematic equations are given as:

$$
\frac{\partial f}{\partial x}=\left(\begin{array}{ccc}
0 & 0 & -v_{u} \sin \theta \\
0 & 0 & -v_{u} \cos \theta \\
0 & 0 & 0
\end{array}\right)
$$

This matrix is updated every time before executing computation of recursive EKF equations, in particular, calculation of prediction model. These predicted states are updated using encoder measurements. Here, we have one encoder located on steering shaft that gives number of pulses for rotation of shaft. The resolution of this encoder is kept to 0.1 degrees, that is, it is capable of measuring change is steering angle to 0.1 degrees assuming steering gear box reduction ratio equal to one. Another encoder is located on driving shaft of rear axle that generates the pulses and the number of pulses per unit time determines the distance traveled in unit time. In our case, the resolution is 1 
degree; means for every one degree rotation of driving wheel one pulse is generated. The counting of pulse and hence the distance as well as steering angle per unit time is carried out by odometry equations as:

$$
\begin{aligned}
& \Delta s=\frac{M \times \pi \times D}{360} \\
& \Delta \delta=\frac{N}{10}
\end{aligned}
$$

where, $\mathrm{M}$ and $\mathrm{N}$ are pulses generated during unit time.

Assuming that the steering angle remains constant for particular computational interval, provided the interval length is short enough, the robot's orientation $\theta$ from one point to another is derived from the incremental change in steering angle $\Delta \delta$, obtained from the number of pulses and equation (3.28). Using these equations the increments in states, $\left[\begin{array}{lll}x & y & \theta\end{array}\right]^{T}$ using odometry results in local frame are given by:

$$
\Delta x=-(r-r \cos \Delta \delta) ; \quad \Delta y=r \sin \Delta \delta
$$

Whereas in global frame on world coordinate frame is given as [27]:

$$
\begin{aligned}
& \Delta x=r \cos \delta[\cos \Delta \delta-1]-r \sin \Delta \delta \sin \delta \\
& \Delta y=r \sin \delta[\cos \Delta \delta-1]-r \sin \Delta \delta \cos \delta
\end{aligned}
$$

Here, $\quad r=\frac{\Delta S}{\Delta \delta}$, sensed radius of curvature along which vehicle takes a turn. 


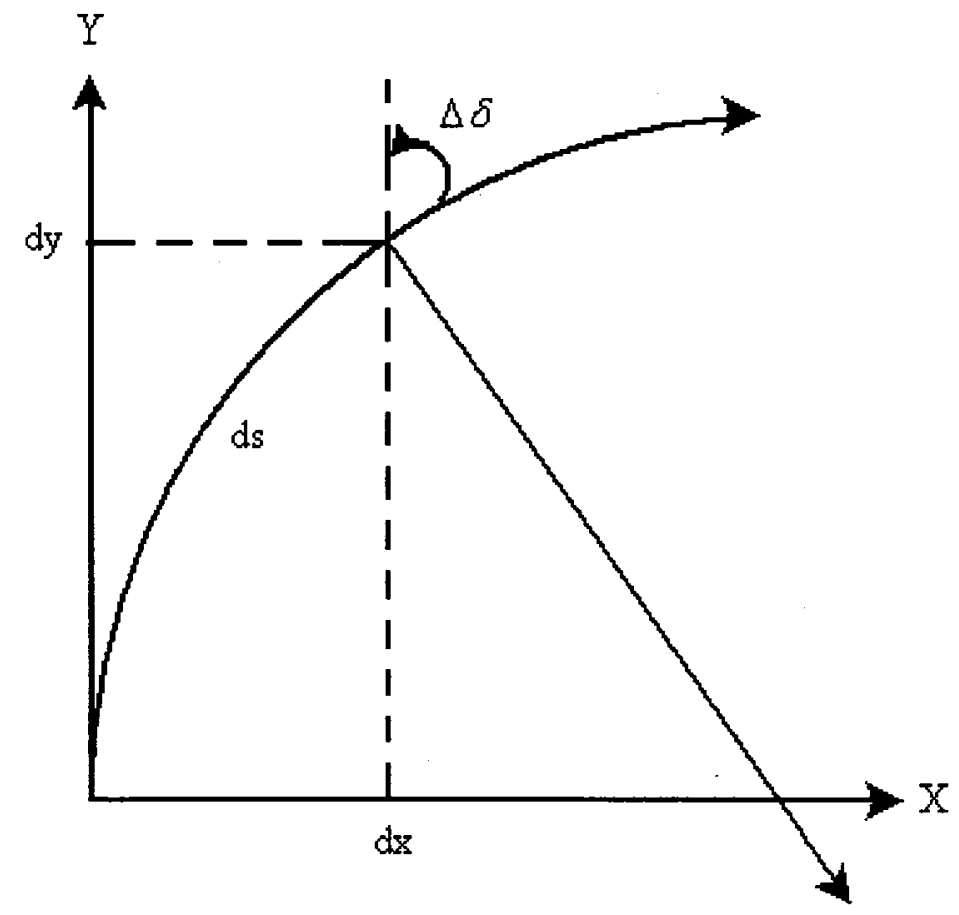

where, $\quad \mathrm{dx}=$ Displacement in $\mathrm{X}$ direction in unit time $\mathrm{dy}=$ Displacement in $\mathrm{Y}$ direction in unit time $\Delta \delta=$ Rate of change in steering angle, $\delta$ $\mathrm{ds}=$ Distance traveled in unit time

Figure 3.5: Dead reckoning geometry.

Hence, the absolute value of steering angle is given as:

$$
\delta_{k}=\delta_{k-1}+\Delta \delta
$$

where, $\delta_{k}=$ steering angle at $\mathrm{k}^{\text {th }}$ step, $\quad \delta_{k-1}=$ steering angle for previous step

The Robot's orientation at a particular step is derived as:

$$
\theta=v_{u} \tan \delta / l
$$

with $l$ being the distance between front and rear wheels.

Since here we need to predict all the three states, the linearized observation model matrix 


$$
\frac{\partial h}{\partial x}=\left(\begin{array}{lll}
1 & 0 & 0 \\
0 & 1 & 0 \\
0 & 0 & 1
\end{array}\right)
$$

There is always presence of noise while measuring the distance and steering angle may be due to robot vibration, slippage and the errors that we saw in previous sections. Since the nature of these noises is unpredictable, the usual practice is to assume it as Gaussian noise. Here we have considered the noise as Gaussian with unit variance. The error between predicated value of states and sensor measurements which is minimized using EKF is shown in figure below:
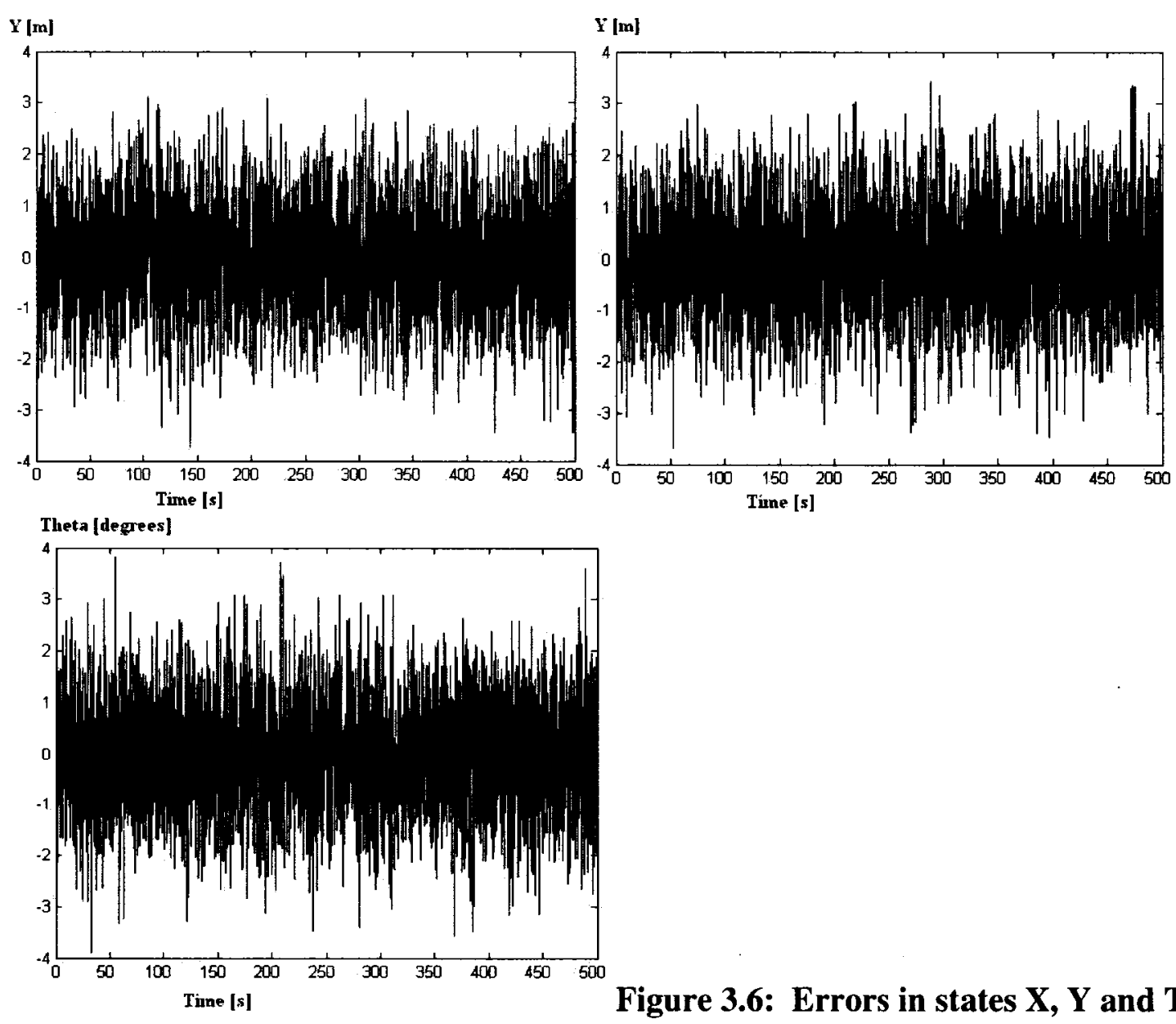

Figure 3.6: Errors in states $X, Y$ and Theta 
From above figures, it can be seen that the errors occurs within the range of $+\mid-4$ units. Due to these errors, different combinations of system noise and sensor noise covariance matrices are taken into consideration and the one which gives better results are adopted for this work.

\begin{tabular}{|c|c|c|}
\hline Serial & Q & R \\
\hline 1 & $\mathrm{I}_{3}$ & $50 . \mathrm{I}_{3}$ \\
\hline 2 & $\mathrm{I}_{3}$ & $200 . \mathrm{I}_{3}$ \\
\hline 3 & $\mathrm{I}_{3}$ & $500 . \mathrm{I}_{3}$ \\
\hline 4 & $\mathrm{I}_{3}$ & $700 . \mathrm{I}_{3}$ \\
\hline 5 & $10 . \mathrm{I}_{3}$ & $50 . \mathrm{I}_{3}$ \\
\hline 6 & $10 . \mathrm{I}_{3}$ & $200 . \mathrm{I}_{3}$ \\
\hline 7 & $10 . \mathrm{I}_{3}$ & $500 . \mathrm{I}_{3}$ \\
\hline 8 & $10 . \mathrm{I}_{3}$ & $700 . \mathrm{I}_{3}$ \\
\hline
\end{tabular}

\section{Table 3.1: Selection for $Q$ and $R$.}

For the first four combinations the system noise covariance matrix is kept to identity while the sensor noise covariance is changing. From the performance results as shown in chapter 7 , out of all the four, second case was found to be most convincing as the states were much closer to the trajectory. But in these cases the system noise covariance is unity which is quite impractical, hence considering the noise covariance matrix as 10x Identity, another four cases are considered. For the first case, the $\mathbf{R}=50 \mathrm{I}_{3}$, the updated states are within the range of $+\mid-0.5$ meters from predicted states. As we move on to $\mathbf{R}=700 \mathrm{I}_{3}$, this range reduces gradually but at the same time the Kalman filter will rely less on sensor 
measurements. Hence the choice is made to keep $\mathbf{R}=500 \mathrm{I}_{3}$ considering some value between $\mathbf{R}=50 \mathrm{I}_{3}$ and $\mathbf{R}=700 \mathrm{I}_{3}$ for this work. With this $\mathbf{R}$, the performance of extended Kalman filter is as shown in figures below:
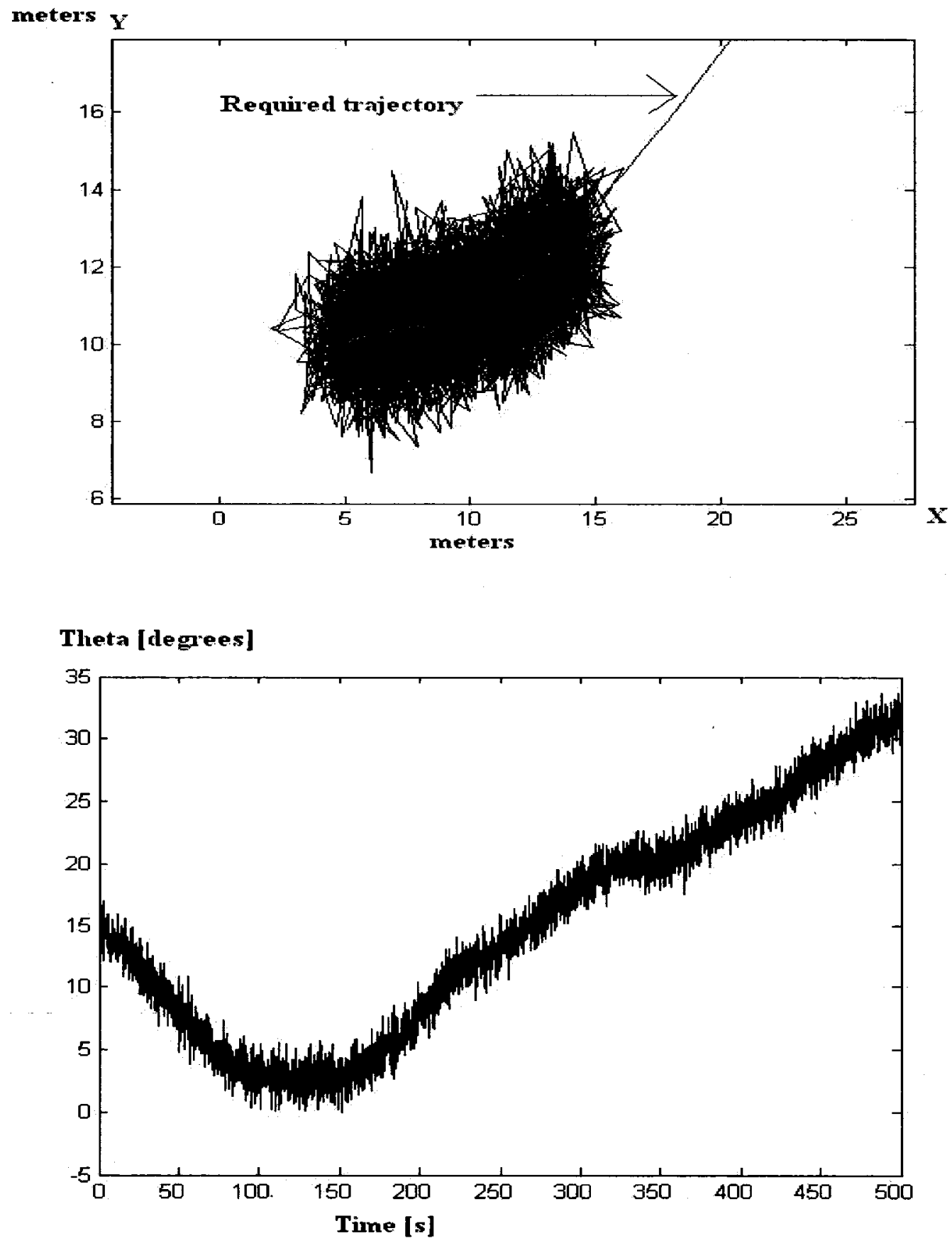

Figure 3.7: States updated using $E K F$ with $R=500 I_{3}$ and $Q=10 I_{3}$. 

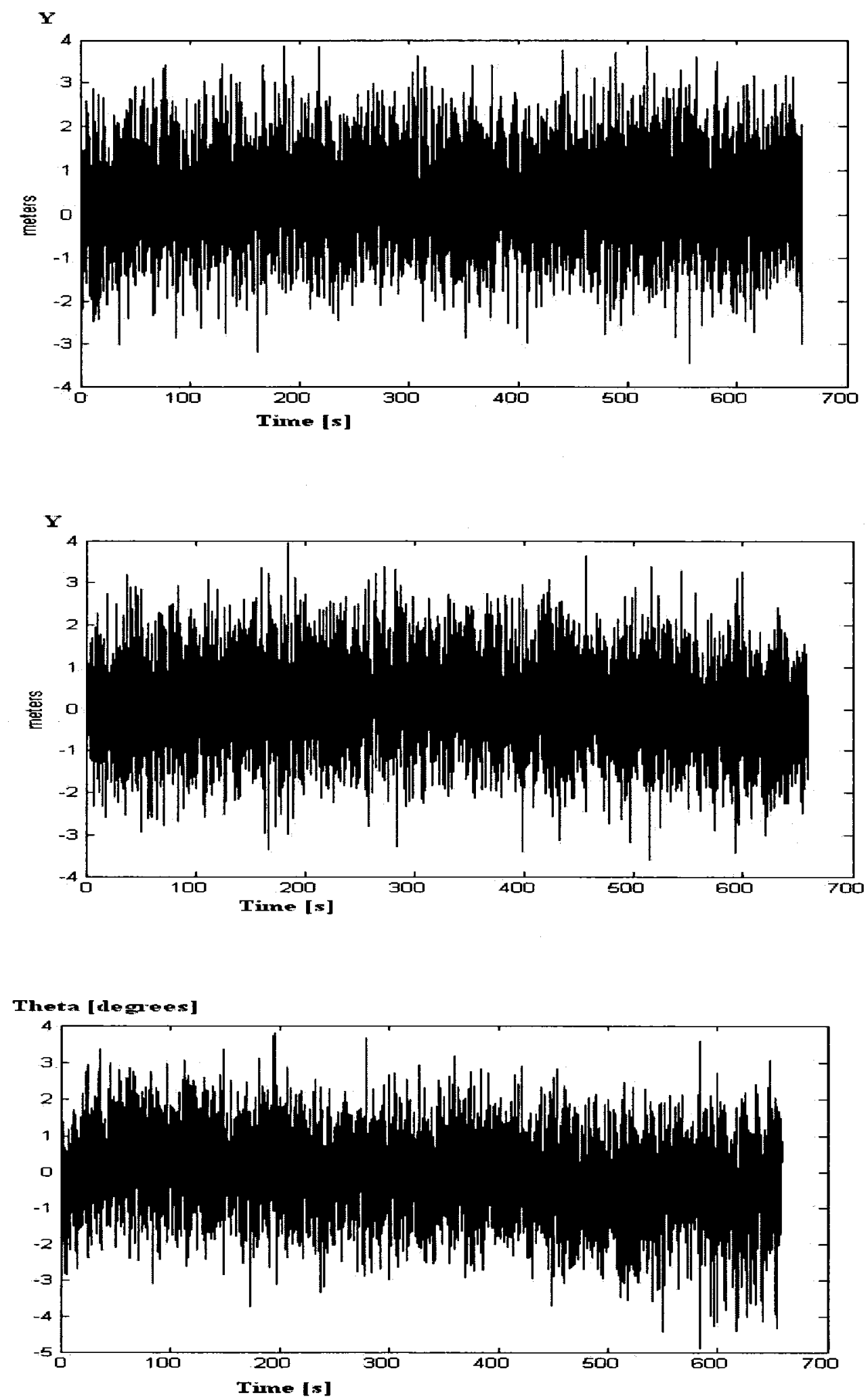

Figure 3.8: Difference between updated and predicted states, $R=200 I_{3}$ and $Q=10 I_{3}$ 


\section{Chapter 4: Mapping}

\subsection{Introduction and literature review for mapping}

One of the most important tasks of an autonomous system of any kind is to acquire knowledge about its environment. In order to widen the range of application of robotic devices, both in industrial and research applications, it is necessary to develop systems with high levels of autonomy and able to operate in unstructured environments with little or no priori information. This can be achieved if the robot has the ability to interact coherently with its environment, by recovering robust and spatial descriptions of environment using information from sensors and efficiently utilizing it recursively to carry out different tasks such as path planning, obstacle avoidance, decision making and other related activities. The data structure that keeps all the information regarding the plan of subsequent paths to be followed is known as map and mapping is the task of generating models of robot environments from sensor data [25]. Mapping is one of the most important problems in world of mobile robots. In some cases, the maps are previously available and are used for localization and later for path planning and obstacle avoidance. But in certain cases where the knowledge of environment is unknown or partially known mapping become important problem to be solved prior to path planning and obstacle avoidance. Even if the map is available of certain indoor environment, surprisingly enough, most buildings do not comply with the blueprints generated by the architects. And even if the blueprints are accurate, they would not contain furniture and other items that, from the robots prospective, determine the shape of the environment just as much as walls and doors. Maps for structured environment are typically 2-D overhead 
views of the building structure, for instance, building blueprints. These environments are assumed to be vertically indistinct, such that the features like walls are flat and texture less. For mapping, it needs a variety of sensors to be able to interact with the real world and mechanisms to extract meaningful information from the data provided. Ability to generate maps online from scratch can greatly reduce the efforts involved in installing a mobile robot and makes it capable of adapting the change without human intervention. "In fact, mapping is one of the core competencies of truly autonomous robots." [24] Learning maps is a "chicken-and- egg" problem, and because of that it is often referred as simultaneous localization and mapping (SLAM) or concurrent mapping and localization problem. In this, first problem is of localization in which when a robot moves through unknown environment, it accumulates errors in odometry, making it gradually less reliable. Second problem is mapping. Constructing a map when the robot's position and orientation are known is relatively easy and is discussed in many literatures [25]. The level of difficulty of mapping problem depends on some factors such as: size of environment, amount of noise in perception and actuation, perceptual ambiguity and number of cycles. In absence of both, robot has to carry out both the tasks, that is, localization and mapping. Localization using odometry and EKF is already discussed in chapter 3. Here we shall see the task of mapping an unknown environment in this chapter.

Maps may be classified as: topological, geometric and grid based. Background for all kinds of maps is discussed in this chapter. In this work we have used grid based methods for its some advantages over topological based methods, for example, they are comparatively easy to build, represent and maintain. 


\subsection{Topological representations}

Topological representations represent the environments with graph-like structures, where nodes correspond to something distinct object or the landmarks and edges represent as adjacency relationship between nodes. For instance, places may be locations with specific distinguishing features, such as intersection of T-junctions, and edges may correspond to specific behaviors or motion commands that enables the robot to move from one location to another, such as wall following. Before going through the discussions of different kinds of roadmap methods, it is necessary to know the properties the roadmaps should have:

Accessibility: there exists a path that connects the start point ' $\mathrm{q}_{\mathrm{start}}$ ' in collision free workspace with some point ' $\mathrm{q}_{\text {start }}^{\mathrm{t}}$ ' that belongs to roadmap.

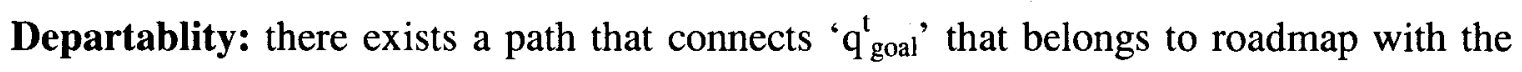
goal point 'qgoal' in collision free workspace.

Connectivity: there exists a path that connects ' $q_{\text {start }}^{\mathrm{t}}$ ' with ' $\mathrm{q}_{\text {goal }}^{\mathrm{t}}$ ' that belongs to the roadmap.

\subsubsection{Visibility graphs}

Visibility graphs are class of roadmaps that consist of nodes that share edges if they are within the sight of each other. The visibility maps should possess the properties of accessibility, departability and connectivity. Connectivity must then explicitly be proved for each map for the structure to be a roadmap. The principle of visibility graph method is to construct a semi-free path as a simple polygonal line connecting the starting point in 
free configuration workspace to the goal point through the vertices of the obstacle workspace. Example for simple polygonal configuration space with start point and a goal point is as shown below:

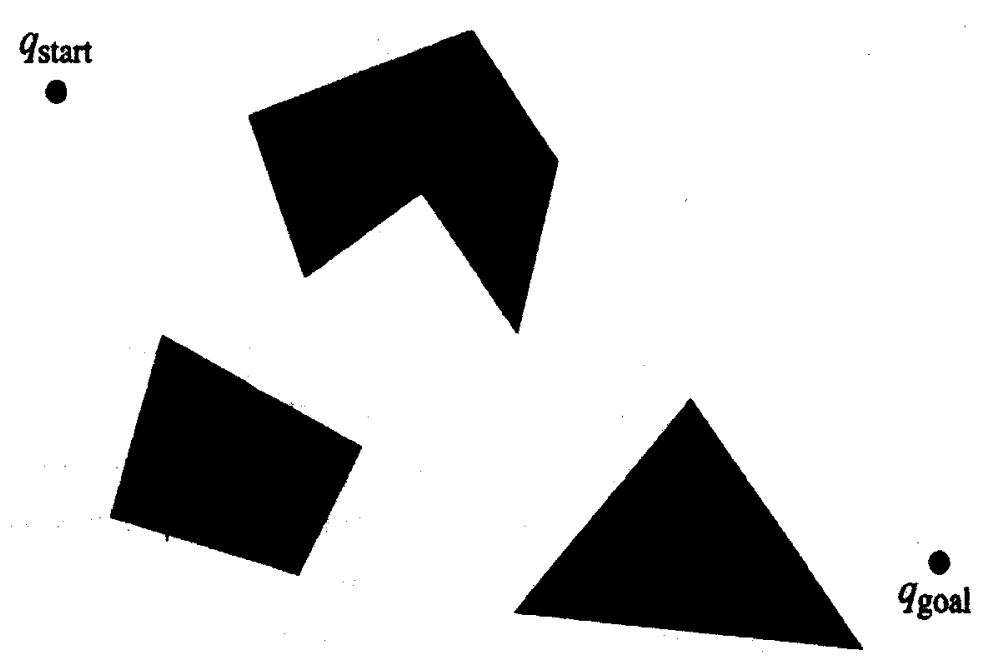

Figure 4.1: Polygonal configuration in space

All the nodes of visibility graph for above configuration includes the goal point, initial point and all the vertices of the obstacles while edges are the lines that connects every nodes with each other provided each nodes are in the sight of a particular node. The visibility graph for the above configurations can be drawn as shown in figure below:

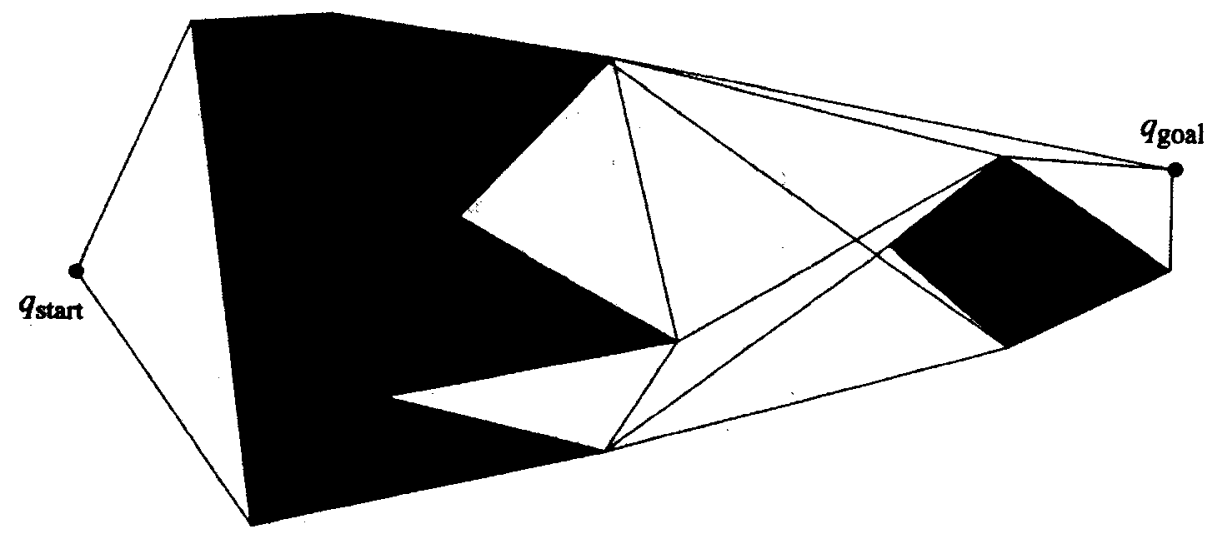

Figure 4.2: Visibility graphs 
After having drawn visibility graph a search for the optimal path that connects the initial configuration with the final configuration in collision free workspace is conducted. Unfortunately, the visibility graph has many unnecessary edges. The use of supporting and separating lines can reduce the number of edges. The supporting line is tangent to the obstacles that lie on the same side of the obstacles. For the non-smooth obstacles as in case shown above with polygons, a supporting line can be tangent to a vertex of the obstacle. A separating line is tangent to two obstacles that the obstacles lie on opposite side of the separating lines. By using these lines the reduced visibility graph is constructed and there by getting rid of those lines that are not necessary and hence imparting the simplicity for optimal roadmap search algorithm. The notion of separating and supporting lines can be used to generalize the visibility graph method for curved obstacles [25]. For constructing the edges, the general method is to check for the presence of intersecting points for the lines connecting each node. If intersecting point exists then it indicates the nodes are not in its direct sight of each other and that line is eliminated from the set of edges generating visibility graphs and for those lines that do not intersect, the nodes corresponding to those edges are considered to be in direct sight of vision and are included in the set of edges. This procedure is carried out for all nodes in a set consisting of vertices of obstacles and the start and goal points. Because of this, the construction of visibility graph leads to great complexity. There is more efficient way to compute the set of vertices that are visible from the set ' $v$ ' of nodes. This algorithm is known in the computational geometry literature as plane sweep algorithm. This algorithm solves a problem by sweeping a line called the sweep line across the plane, pausing at each of the vertices of the obstacles. At each vertex, the algorithm updates a 
partial solution to the problem. Plane sweep algorithms are used to efficiently compute the intersections of the line segments in the plane, as discussed above, to generate visibility graph. The algorithm for the visibility graph method is as [5]:

1. Construct the visibility graph.

2. Search for a path from start point ' $\mathrm{q}_{\text {start }}$ ' to goal point ' $\mathrm{q}_{\mathrm{goal}}$ '.

3. If a path is fount, return it; otherwise, indicate failure.

\subsubsection{Generalized Voronoi diagrams or Retraction approach}

Voronoi diagram was originally defined as the set of points where the distance from two closest obstacles is same. In this case, during path planning the motion is achieved by first moving away from the closet points to the obstacle to Generalized Voronoi (GV) diagrams, then along the double equidistant Voronoi diagram to the vicinity of the goal, and then from Voronoi diagram to the goal point. Thus, it can be said as a Voronoi diagram has the property of accessibility, connectivity and departability as discussed earlier. Simple example of Voronoi diagram is as shown below: 


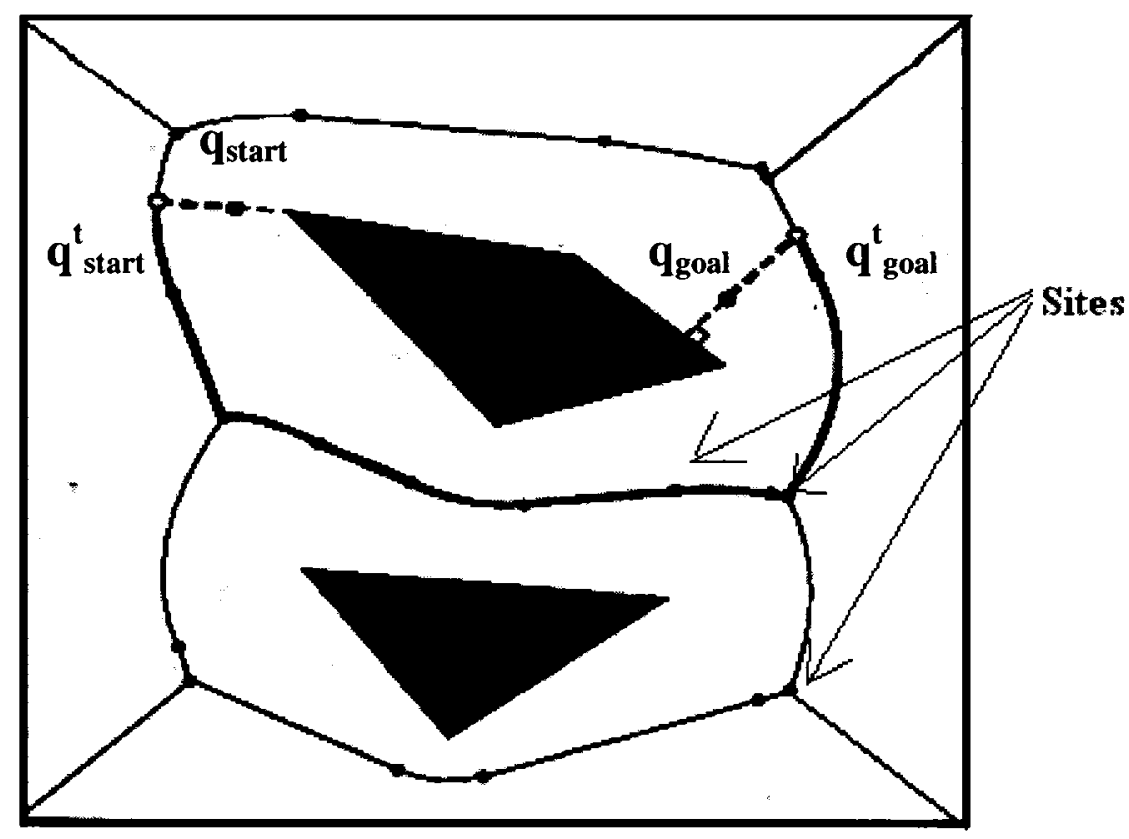

Figure 4.3: Generalized Voronoi diagram.

The Voronoi diagram is defined for a set of points called sites [26]. A Voronoi diagram is the set of points closest to a particular site [26]. The Voronoi diagram is then the set of points equidistant to two sites, it sections off the free space into regions that are closest to particular site. Points on the Voronoi diagram have two closest sites. In the planner case, the Voronoi diagram is a collection of line segments. This method is also known as deformation retracts.

\subsection{Geometric representation}

Geometric model use geometric primitives for representing the environment. The next step is to do mapping that involves estimating the parameters of primitives to best fit the sensor observations. Line segments are used by many researchers [27] for the 
representation of the environment. The major drawback of the geometric approach is the number of different data structures for each geometric primitive that the mapping system must handle, such as, segments, polygons, ellipses, etc. Also, taking into account the uncertainty of the sensor measurements for each sequence of different primitives is very complex.

\subsection{Occupancy grids representations}

In past couple of decades, occupancy grid maps have become a dominant paradigm for environment modeling in mobile robots. Occupancy grid maps are spatial representations of robot environment. They represent environments surrounded by mobile robots using fine grained, metric grids of variables that reflect the occupancy of the environment. Once achieved, they enable various key functions necessary for mobile robot navigation, such as localization, path planning, collision avoidance, etc. At the end of the 1980s, Elfes [28] introduced a new framework to multi-sensor fusion called occupancy grids. This used sonar range sensor for interpreting the environment. It is a stochastic tessellated representation of spatial information that maintains probabilistic estimates of the occupancy state of each cell in a lattice [28].Other most popular and successful methods of accounting for uncertainty are given by (Elfes, 1990, 1992a; Moravec and Blackwell, 1992). This basic algorithm suffers a key problem [29], in which it decomposes the highdimensional mapping problem into a collection of one-dimensional problems, where the occupancy of each grid cell is estimated independently. This includes conflicts that may lead to inconsistent maps, even for noise free sensors. The alternative algorithm was 
proposed by Sebastian Thrun in [29] that employs the expectation maximization algorithm for searching maps that maximize the likelihood of the sensor measurements. Here, we shall discuss the basic algorithm and the same has been implemented in this thesis for representation of environment around car-like mobile robot. Standard occupancy methods are characterized by two algorithm choices: [29]

1) They decompose the high-dimensional mapping problem into many binary estimation problems, which are then solved independently of each other.

2) They rely on inverse model of the robot's sensors which reasons from sensor measurements to maps.

"The occupancy grid is a multidimensional random field that maintains stochastic estimates of the occupancy state of the cells in spatial lattice' [28]. A grid based maps represents the environment by splitting it into large number of small cells. All these cells are uniformly sized and are usually fixed during the exploration of environment. Each cell represents the probability that the corresponding region in the environment is occupied. Occupancy values range from zero to one. An occupancy value near zero $p(c e l l=o c c)=0$ corresponds to an open cell and indicates with highest probability that the corresponding region is free of any obstacles. An occupancy value near one $p($ cell $=o c c)=1$ indicates the opposite or that the region is occupied. When no previous knowledge is available the occupancy value of each grid is initialized at 0.5 indicating the presence or absence of obstacle in that particular grid cell is equally likely. Once all grids are initialized, they are updated recursively to construct a sensor- derived map of the robot's world by interpreting the incoming range readings using probabilistic sensor models. For this, the basic assumption made that the location of mobile robot is 
always known. Incrementally updating of these occupancy grids using readings taken from sensor is done using Bayesian estimation procedure.

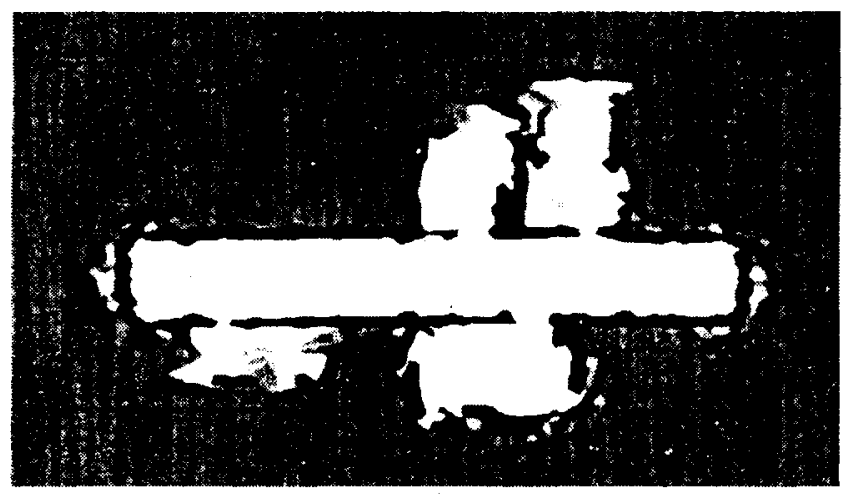

Figure 4.4: Occupancy probability map of an office environment built from sonar measurements. [24]

\subsection{Brief comparison between topological and grid based methods}

As seen earlier, grid based approach indicates presence or absence of obstacles by assigning each grid probabilities. Occupancy grids are easy to construct and to maintain large scale environment. It differentiates different places using robot's geometric position which is determined incrementally using readings from sensors and odometry. It helps in sensor integration and thereby updating same occupancy grid by combining different posterior densities or sensor models operating independently. As far as robot's pose is known, different situations with similar sensor readings are disambiguated. While it suffers from complexity and the accuracy of occupancy grid based environment depends on the grids resolution or size of grids. On the other hand, topological methods represents environment by graphs using nodes and arcs connecting each nodes if there exists direct 
connectivity between them. It determines the location of robot relative to the model primarily based on landmarks or distinct, momentarily sensor features. It is compact, permits fast path planning, facilitates interfacing to symbolic planners and problem solvers and allows human interface. The advantages and disadvantages of both paradigms are tabulated below:

\begin{tabular}{|c|c|}
\hline Grid-based (metric) approaches & Topological approaches \\
\hline $\begin{array}{l}+ \text { easy to build, represent, and maintain } \\
+ \text { recognition of places (based on geometry) is non- } \\
\text { ambiguous and view point-independent }\end{array}$ & $\begin{array}{l}\text { permits efficient planning, low space complexity } \\
\text { (resolution depends on the complexity of the en- } \\
\text { vironment) }\end{array}$ \\
\hline \multirow[t]{2}{*}{+ facilitates computation of shortest paths } & $\begin{array}{l}+ \text { does not require accurate determination of the } \\
\text { robot's position }\end{array}$ \\
\hline & $\begin{array}{l}+ \text { convenient representation for symbolic plan- } \\
\text { ner/problem solver, natural language }\end{array}$ \\
\hline $\begin{array}{l}\text { - planning inefficient, space-consuming (resolution } \\
\text { does not depend on the complexity of the envi- } \\
\text { ronment) } \\
\text { - requires accurate determination of the robot's po- }\end{array}$ & $\begin{array}{l}\text { - difficult to construct and maintain in large-scale } \\
\text { environments if sensor information is ambiguous } \\
\text { - recognition of places often difficult, sensitive to } \\
\text { the point of view }\end{array}$ \\
\hline - poor interface for most symbolic problem solvers & \\
\hline
\end{tabular}

Table 4.1: Advantages and disadvantages of grid based and topological approaches [30]

\subsection{Occupancy grid development for mobile robot navigation}

Consider, $m$ be the occupancy grid map and let $m_{x, y}$ denote the occupancy of the grid cell with index $\langle x, y\rangle$. Let $r_{1}, r_{2}, \ldots, r_{t}$ are the range readings available at each time step from laser range finder, where each measurement carries information about the 
occupancy of many grid cells. Hence, the problem of occupancy grid mapping is to determine the probability of each grid cell $m$ using the measurements $r_{1}, r_{2}, \ldots, r_{t}$, that is, $p\left(m_{x, y} \mid r_{1}, r_{2}, \ldots, r_{t}\right)$ and is given as using Bayesian formula [26]:

$$
p\left(m_{x, y} \mid r_{1}, r_{2}, \ldots, r_{t}\right)=\frac{p\left(r_{t} \mid r_{1}, r_{2}, \ldots, r_{t-1}, m_{x, y}\right) \cdot p\left(m_{x, y} \mid r_{1}, r_{2}, \ldots, r_{t-1}\right)}{p\left(r_{t} \mid r_{1}, r_{2}, \ldots, r_{t-1}\right)}
$$

Since, the occupancy grid is modeled as a Markov random field of order 0 , so the individual cell states can be estimated as independent random variables, also the past sensor readings are conditionally independent given the knowledge of map $m$, for any

time. Hence, $\quad p\left(m_{x, y} \mid r_{1}, r_{2}, \ldots, r_{t}\right)=\frac{p\left(r_{t} \mid m_{x, y}\right) \cdot p\left(m_{x, y} \mid r_{t-1}\right)}{p\left(r_{t} \mid r_{t-1}\right)}$

The term $p\left(r_{t} \mid m_{x, y}\right)$ in above equation is derived at each time of each cell and applying Bayes rule we can rewrite it as:

$$
p\left(r_{t} \mid m_{x, y}\right)=\frac{p\left(m_{x, y} \mid r_{t}\right) \cdot p\left(r_{t}\right)}{p\left(m_{x, y}\right)}
$$

Now, let $z$ be the true parameter space range value then the sensor probability density function for 2-dimensional case with Gaussian uncertainty, is given as:

$$
p(r \mid z)=\frac{1}{\sqrt{2 \pi} \sigma_{r} \sigma_{\theta}} \exp \left[-\frac{1}{2}\left(\frac{(r-z)^{2}}{\sigma_{r}^{2}}+\frac{\theta^{2}}{\sigma_{\theta}^{2}}\right)\right]
$$

For simplicity in calculation, it is normal practice to calculate "log-odds" instead of estimating posterior $p\left(m_{x, y} \mid r_{1}, r_{2}, \ldots, r_{t}\right)$, and is defined as: 


$$
l_{x, y}=\log \frac{p\left(m_{x, y} \mid r_{1}, r_{2}, \ldots, r_{t}\right)}{1-p\left(m_{x, y} \mid r_{1}, r_{2}, \ldots, r_{t}\right)}
$$

The log-odds can take any real value and from it we can easily retrieve the posterior occupancy probability as:

$$
p\left(m_{x, y} \mid r_{1}, r_{2}, \ldots, r_{t}\right)=1-\left[\exp \left(l_{x, y}\right)\right]^{-1}
$$

Equation (4.2) can be written in the similar way for the posterior probability that the grid cell is not occupied or free:

$$
p\left(\bar{m}_{x, y} \mid r_{1}, r_{2}, \ldots, r_{t}\right)=\frac{p\left(r_{t} \mid \bar{m}_{x, y}\right) \cdot p\left(\bar{m}_{x, y} \mid r_{t-1}\right)}{p\left(r_{t} \mid r_{t-1}\right)}
$$

Now, applying the Bayes rule as shown by equation (4.3) and dividing equation (4.2) by (4.7), we have:

$$
\frac{p\left(m_{x, y} \mid r_{1}, r_{2}, \ldots, r_{t}\right)}{p\left(\bar{m}_{x, y} \mid r_{1}, r_{2}, \ldots, r_{t}\right)}=\frac{p\left(m_{x, y} \mid r_{t}\right) \cdot p\left(\bar{m}_{x, y}\right) \cdot p\left(m_{x, y} \mid r_{t-1}\right)}{p\left(\bar{m}_{x, y} \mid r_{t}\right) \cdot p\left(m_{x, y}\right) \cdot p\left(\bar{m}_{x, y} \mid r_{t-1}\right)}
$$

Expressing $p\left(\bar{m}_{x, y}\right)$ as $1-p\left(m_{x, y}\right)$ and rewriting equation (4.8) as,

$$
\frac{p\left(m_{x, y} \mid r_{1}, r_{2}, \ldots, r_{t}\right)}{1-p\left(m_{x, y} \mid r_{1}, r_{2}, \ldots, r_{t}\right)}=\frac{p\left(m_{x, y} \mid r_{t}\right) \cdot\left(1-p\left(m_{x, y}\right)\right) \cdot p\left(m_{x, y} \mid r_{t-1}\right)}{\left(1-p\left(m_{x, y} \mid r_{t}\right)\right) \cdot p\left(m_{x, y}\right) \cdot\left(1-p\left(m_{x, y} \mid r_{t-1}\right)\right)}
$$

Now, after applying log on both sides and substituting the terms in the form of log-odds as shown in equation (4.5), we have:

$$
l_{x, y, t}=\log \frac{p\left(m_{x, y} \mid r_{t}\right)}{1-p\left(m_{x, y} \mid r_{t}\right)}+\log \frac{1-p\left(m_{x, y}\right)}{p\left(m_{x, y}\right)}+l_{x, y, t-1}
$$


Where, $l_{x, y, t-1}$ is the log-odds of previous step and $p\left(m_{x, y}\right)$ is the initial probability value selected for each grid cell. Here, it is assumed to be 0.5 . Its initial value at time $t=0$ is given by equation (4.5). $p\left(m_{x, y} \mid r_{t}\right)$ is the inverse sensor model, since it maps sensor measurement back to its cause.

The desired probability can also be calculated in following way [30]:

$p\left(m_{x, y} \mid r_{1}, r_{2}, \ldots, r_{t}\right)=1-\left[1+\frac{p\left(m_{x, y} \mid r_{t=1}\right)}{1-p\left(m_{x, y} \mid r_{t=1}\right)} \prod_{\tau=2}^{T} \frac{p\left(m_{x, y} \mid r_{t=\tau}\right)}{1-p\left(m_{x, y} \mid r_{t=\tau}\right)} \cdot \frac{1-p\left(m_{x, y}\right)}{p\left(m_{x, y}\right)}\right]^{-1}$

Above equations shows the method to increment each grid recursively when each sweep of laser range finder is scanned. Based on assumption of the 2-dimensional environment representation, we consider the cross-section of the navigation area represents the overall structure of surrounding environment. Therefore, the set of laser readings used here are those derived from the intersection of horizontal plane and the vertical planes of the environment. These readings are represented by 2 dimensional coordinates with respect to robots frame of reference and provides information that is represented as pairs $(\rho, \zeta)$ of polar coordinates where $\rho$ is the range or distance measured from the object to the sensor and $\zeta$ is the azimuth angle between the direction of been corresponding to particular $\rho$ with respect to its local frame. In this work the maximum range is fixed to 20 meters and the angle of visibility is 180 degrees. Each time the laser beam is swept along horizontal plane through visibility angle of 180 degrees giving the set of pairs at definite resolution. The sensor angular resolution is taken as 1 degree and hence, number of pairs is, 


$$
N=\frac{\text { Visibility }}{\operatorname{Re} \text { solution }}+1=\frac{180}{1}+1=180+1=181
$$

The set of pairs obtained are always corrupted with presence of noise in practical case and has to be eliminated before taken into actual use for occupancy grid development. In case of indoor environments, the level of noise is low as compared to mining environment or with loose sand. The characteristics of noise are to be calibrated through experiments. All these are to be filtered using extended Kalman filter. The basis equations for EKF are shown in chapter 3. Here, we are using two separate EKFs to estimate the values of range and azimuth angle respectively. The noise variances for range and azimuth angle is as given below, which is given in [31],

\section{Range error variance:}

Mean and standard deviation is given as:

Mean: $E_{\rho}=\frac{1}{N} \sum_{i=1}^{N} \rho_{i}$

Standard deviation: $\sigma_{\rho}=\frac{1}{N-1} \sum_{i=1}^{N}\left(\rho_{i}-E_{\rho}\right)$

The range variance is given as:

$$
R_{\rho}=\sigma_{\rho}^{2}=\left(9.6 \times 10^{-7} \rho^{2}-5.6 \times 10^{-4} \rho+21.213 \mathrm{~mm}\right)^{2}
$$

The azimuth angle variance is given as: $\quad R_{\zeta}=\sigma_{\zeta}^{2}=\left(\frac{\Delta \alpha}{4}\right)^{2}$

Using this error variance, the noise is filtered out from the range readings using Extended 
Kalman filter. The states equations for expected value of range is $\left[\begin{array}{l}\rho \\ \frac{\partial \rho}{\partial t}\end{array}\right]$. The linearized dynamic matrix for prediction model is given as:

$\mathbf{F}_{\rho}=\left(\begin{array}{cc}1 & \Delta t \\ 0 & 1\end{array}\right)$, where, $\Delta t$ is the sampling interval and the linearized perception model is given as: $\mathbf{H}=\left[\begin{array}{ll}1 & 0\end{array}\right]$

The corresponding covariance matrices are:

$\mathbf{Q}_{\rho}=\left(\begin{array}{cc}\frac{\Delta t^{4}}{4} & \frac{\Delta t^{3}}{2} \\ \frac{\Delta t^{3}}{2} & \Delta t^{2}\end{array}\right) \sigma^{2} q, \quad$ where $\sigma^{2} q$ is experimentally tuned and is kept equal to one in this case and $\mathbf{Q}_{\zeta}=1$

After filtering out these range and azimuth angle measurements, they are used to update the probabilities of the grids sequentially. The algorithm implemented for updating probabilities using inverse sensor model is given below. 


\section{Algorithm implemented for inverse sensor model:}

Step 1: Initialization of the entire grid in workspace to probabilities 0.5 .

Step 2: $\quad$ For all grid points,

Calculate $R=\sqrt{\left(x-x_{i}\right)^{2}+\left(y-y_{i}\right)^{2}}, x_{i}$ and $y_{i}$ being each grid point and $x$ and $y$ is robot pose and $\phi$ be the angle of each grid with respect $\mathrm{t}$ robots frame.

Step 3: $\quad$ Counting the number of grids that makes and some angle within specified range with respect to robot frame and within maximum range of 20 meters.

Step 4: $\quad$ For all grid points from step 2

If $(\mathrm{R}>\mathrm{Z})$ or $\left(\mathrm{R}<=\mathrm{Z}\right.$ and $\left.|\theta-\Phi|>\tan ^{-1}\left(\frac{2}{Z}\right)\right), \mathrm{Z}$ and $\Phi$ are measurement.

Unchanged probability of that grid

Else if $\left(\mathrm{R}<=\mathrm{Z}\right.$ and $\left.|\theta-\Phi|<=\tan ^{-1}\left(\frac{2}{Z}\right)\right)$

Assign probability 1 .

Else

Assign probability 0.

End.

Step 5: $\quad$ For all grids from step 4 with probability 1

End.

Update probabilities using Bayesian formula.

After performing the probability calculations using the algorithm shown above, decision is made that particular grid is occupied or empty. If that grid is found occupied then it is counted as anstacle region which is further used for obstacle avoidance and will be discussed in chapter 6 . Using this algorithm consider a presence of obstacle in workspace as shown in figure. 


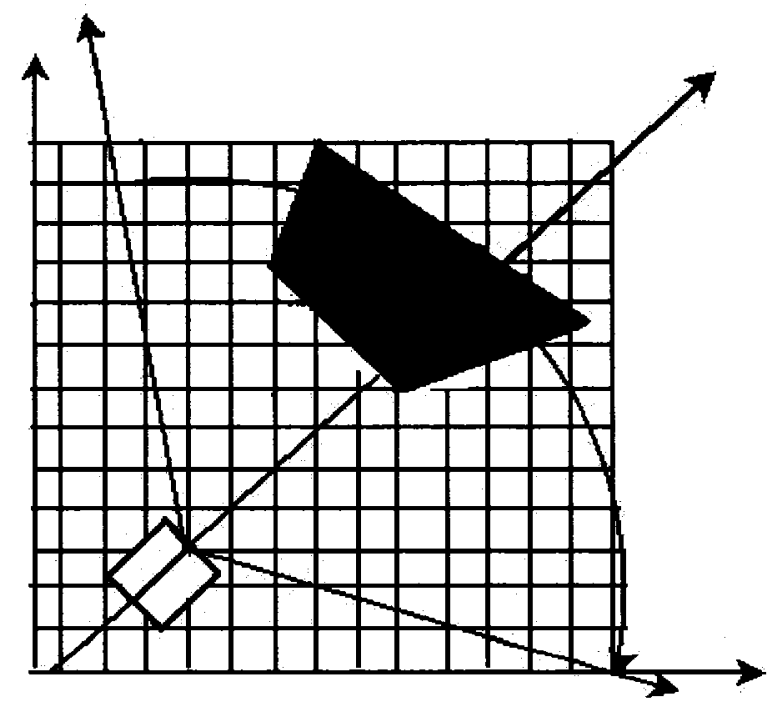

(a)

(b)

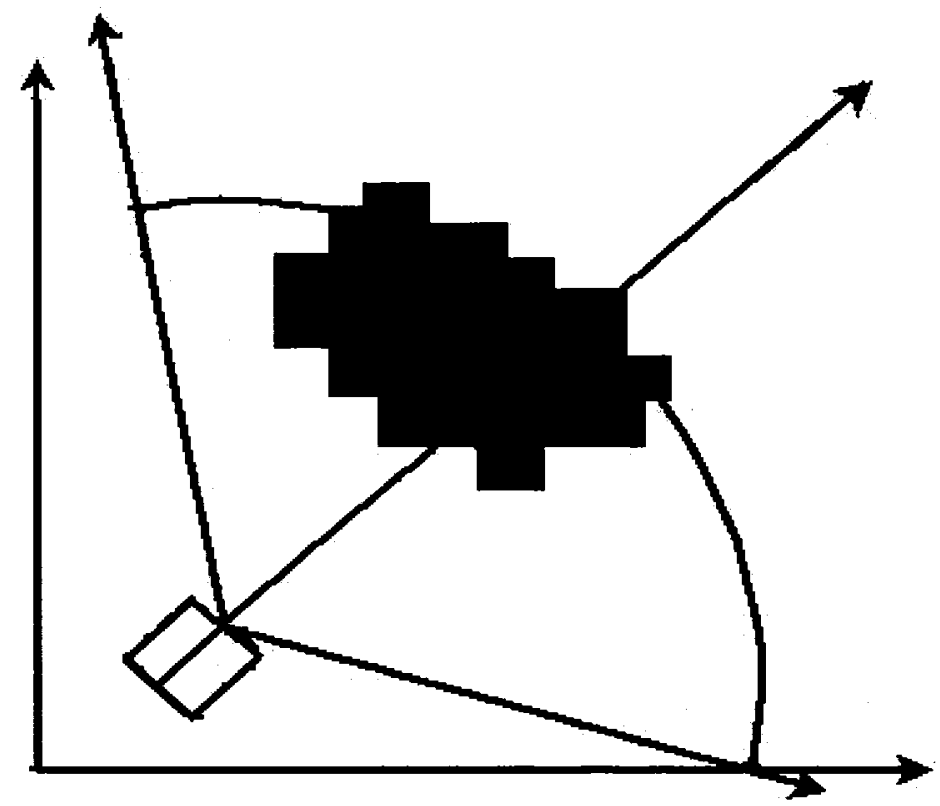

Figure 4.5: Generation of occupancy grid

As shown in figure 4.5 (a), is the first step of algorithm where it counts and consider only those grids within the fixed angular range. In this work it is fixed to $+\mid-65$ degrees about the robots longitudinal axis. Figure 4.5 (b) shows the grids that are occupied with objects as decided in final step of algorithm. All these object occupied grids are then further used by planner to generate path for avoiding obstacle. 


\section{Chapter 5: Path tracking for car-like robots}

This chapter deals with the dynamic model development for mobile car-like robot and development to trajectory tracking controllers so as to maneuver the vehicle on pre determined trajectory. Third section is development of dynamic model; fourth section includes the design of steering and propulsion controllers that use linear quadratic controller. The performance of controller with different operating parameters is also considered.

\subsection{Background of path tracking for car-like robot}

Trajectory tracking for nonholonomic systems with forward and bounded curvature motion due to presence of kinematic constraints is different from holonomic mechanical systems and omni directional robots. Hence the simple controller for such systems may not be directly applicable to constrained mechanical systems because it is not possible that bounded curvature paths are integrated to position level. In path tracking the planner is provided with the reference trajectory that the vehicle has to follow, before the vehicle is put into its execution. It is assumed that the reference trajectory is free from and buildings or fixed structures and the presence of which is absolutely known in prior by the programmer. The trajectory consists of set of equidistant points connected by arcs or lines. The size of this set of points depends on the selection of final time to reach the destination point and sampling rate. This set of points is fed to path planner so as to maneuver the vehicle to desired trajectory. These points on trajectory carry information 
of orientation in addition to its location in terms of Cartesian coordinates in horizontal plane. Since in this work, the vehicle is supposed to move with constant velocity, velocity associated with each point is the same. At every stage of tracking, the planner decides one of these points as a goal point, so as the vehicle has to reach the goal point configuration. The difference in current configuration and desired configuration is error or offset and using these offsets, path tracking is ensured by means of linear, timeinvariant and decoupled controller whose gains may be determined using PID controller or state feedback techniques. Two controllers are used, first for control of steering force to allow the vehicle to move along required direction and second a propulsion controller to maintain vehicle motion with required velocity. The selection of goal point on the trajectory is critical. The distance from current robots position to goal point on trajectory is known as "lookahead" distance [33]. If lookahead distance is more then there are always some possibilities of cutting corners of the obstacle nearby trajectory and the robot may deviate from the predefined path. Hence, for every different trajectory the selection of goal point is different. So, while feeding the trajectory information, criteria for selection of goal point should be given. Also, after the collision avoidance is complete, when the robot has to return to its original path, should look for some goal point on the path because, tracking controllers performs efficiently as long as robot pose is close to path. 


\subsection{Kinematics and Dynamics concepts}

In chapter 2, basic constraints related to holonomic and nonholonomic systems were introduced. Here we will see some constraints related to kinematics and dynamics of mechanical systems.

\section{Kinematics:}

Consider a mechanical system, such a car-like robot here in this case, having a single rigid body. To this we attach a frame of reference and describe the position and orientation vector $\mathbf{x}=\left[\begin{array}{ll}c & \zeta\end{array}\right]$, where $c$ is the coordinates of the origin of vehicles frame and $\zeta$ is the orientation of the frame. The velocity vector with respect to frame is given as $\mathbf{V}=\left[\begin{array}{ll}v & \omega\end{array}\right]$, where $v$ is the linear velocity and $\omega$ is the angular velocity. The angular velocity matrix is given as [4]:

$$
\mathbf{G}(\omega)=\left(\begin{array}{ccc}
0 & -\omega_{z} & \omega_{x} \\
\omega_{z} & 0 & -\omega_{y} \\
-\omega_{x} & \omega_{y} & 0
\end{array}\right)
$$

in most applications, the mechanical systems should satisfy the set of position and orientations constraints which are holonomic constraints. These constraints can be modeled in terms of a set of scalar equations of the form:

$$
h_{i}(x)=0 \quad \mathrm{i}=1,2, \ldots, \mathrm{p}
$$

Where, $\mathrm{p}$ is number of holonomic constraints.

Hence, the allowable values of $x$ can be written in terms of generalized coordinates as,

$$
x=f(q)
$$


Where, $q=6-p, p$ is number of holonomic constraints.

From above two equations (5.2) and (5.3), it can be inferred as:

$$
v=\mathbf{J}_{h}(q) \dot{q}
$$

$\dot{q}$ is velocity vector in configuration space and $\mathbf{J}_{h}$ is the Jacobian matrix and the columns of which span the subspace of velocities.

Here, $\mathbf{J}_{n h}$ is Jacobian matrix, whose columns span the subspace of allowable velocities. In addition to these holonomic constraints the vector $v$ must also satisfy the constraints of the form [5],

$$
g(x) v=0
$$

This equation of the form is known as nonholonomic constraints

Taking into account equations (5.3), (5.4), (5.5),

$$
A(q) \dot{q}=0
$$

Where, $A(q)=g(f(q)) J_{h}(q)$

Hence $\dot{q}$ can be expressed as:

$$
\dot{q}=\mathbf{J}_{n h}(q) a
$$

\section{Dynamics:}

In order to consider the dynamics of mechanical systems, consider,

$v, \dot{v}=$ linear velocity and acceleration of centre of mass respectively

$\omega, \dot{\omega}=$ angular velocity and acceleration of centre of mass respectively

$f, t=$ force and moment at centre of gravity respectively

$m, j=$ mass and moment of inertia of car-like robot.

Assuming the workspace is an inertia system, 


$$
\begin{aligned}
& f=m \dot{v}+m T(\omega) v \\
& t=T(\omega) j \omega+j \omega
\end{aligned}
$$

The above equations can be rewritten as:

$$
\begin{aligned}
& \dot{v}=\frac{f}{m}-T(\omega) v \\
& \dot{\omega}=\frac{(t-T(\omega) j \omega)}{j}
\end{aligned}
$$

\subsection{Model development of car-like robot}

Consider a car-like robot with a pair of rear driving and front steering wheels. The difference between the orientations of front and the rear wheels are assumed to be negligible and the vehicle's dynamics can be analyzed by replacing these wheels with centered located wheel at both axles. The position of the vehicle is marked by its center of mass in terms of Cartesian coordinates $(x, y)$ along horizontal plane and its orientation $\theta$ with respect to reference $\mathrm{X}$ axis. The steering angle is defined as $\delta$ and is the angle made by steering wheels with vehicles longitudinal axis. The velocity components are defined as $\left(v_{u}, v_{w}\right)$ with $v_{u}$ as longitudinal velocity component and $v_{w}$ as lateral velocity component. Angular velocity is given as $\omega$. Let $a$ and $b$, be the distances from

front and rear axles to centre of gravity of vehicle, $l$ be the wheel base, with $m$ and $J$, the mass and yaw moment of inertia respectively.

In order to consider the dynamics of car-like robots, consider the figure shown below: 


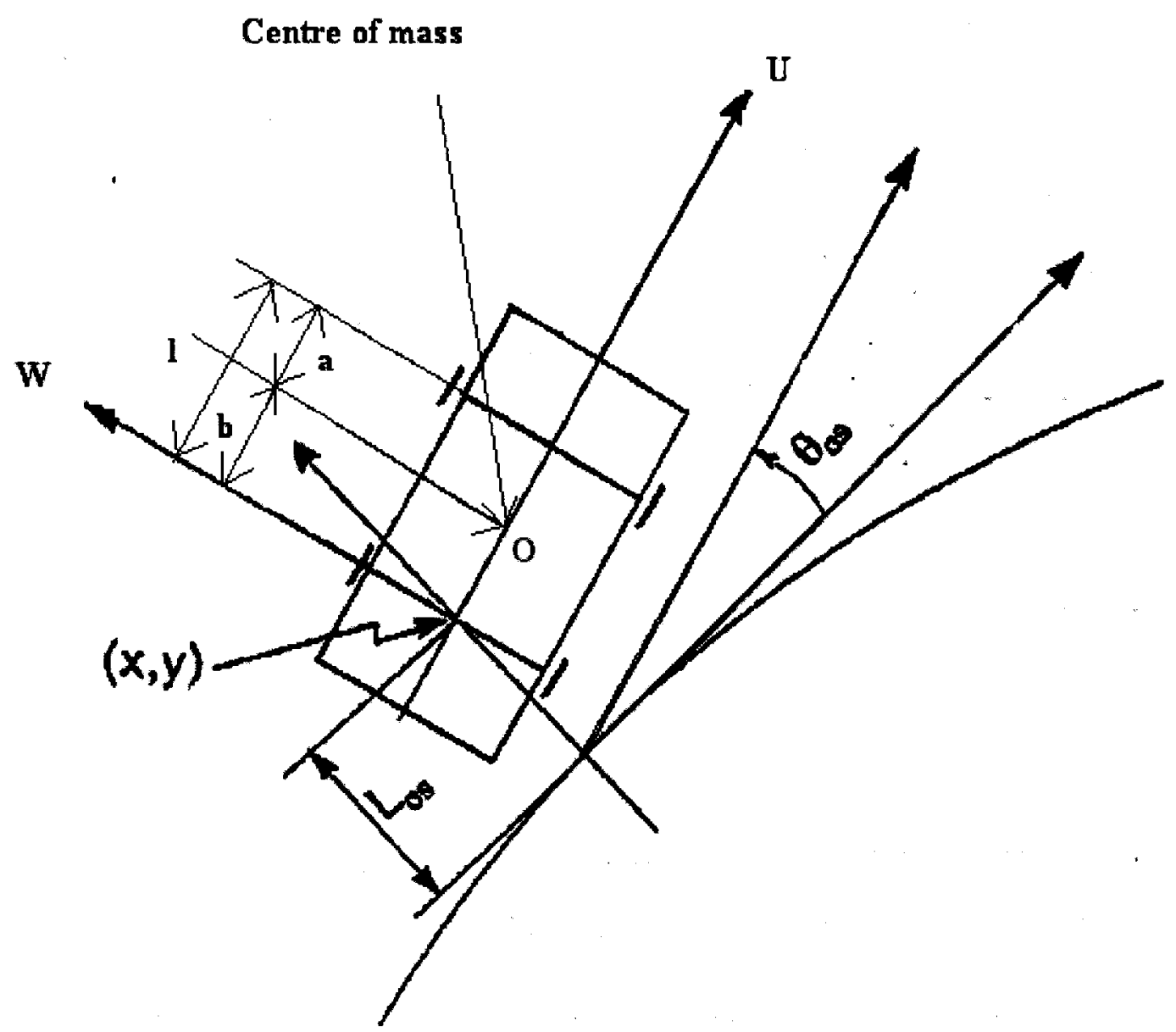

Figure 5.1 Car-like robot

The dynamics of car-like robot is given by [34]:

$$
\begin{gathered}
\dot{x}=v_{u} \cos \theta-v_{w} \sin \theta \\
\dot{y}=v_{u} \sin \theta+v_{w} \cos \theta \\
\dot{\theta}=\omega=\left(v_{u} / L\right) \times \tan \delta \\
\dot{v}_{u}=\dot{v}_{w} \cdot \omega+\frac{F u_{1} \cos \delta}{M}-\frac{F w_{1} \sin \delta}{M}+\frac{F u_{2}}{M}+\frac{F p}{M} \\
v_{w}=-v_{u} \cdot \omega+\frac{F u_{1} \sin \delta}{M}+\frac{F w_{1} \cos \delta}{M}+\frac{F w_{2}}{M}
\end{gathered}
$$




$$
\dot{\omega}=\frac{F u_{1} a \sin \delta}{M}+\frac{F w_{1} a \cos \delta}{M}-\frac{F w_{2}}{M} b
$$

Under the slippage free motion, the velocity is subjected to constraints $\mathbf{J}$, where $\mathbf{J}=\mathbf{J}_{h} . \mathbf{J}_{n h} . \quad \mathbf{J}_{h}$ is matrix of holonomic constraints and $\mathbf{J}_{n h}$ is the matrix of nonholonomic constraints.

Hence,

$$
v=\mathbf{J} v_{u}
$$

where,

$$
\mathbf{J}=\left(\begin{array}{c}
1 \\
\frac{b \tan \delta}{L} \\
\frac{\tan \delta}{L}
\end{array}\right) \quad \text { and } \quad \delta=\tan ^{-1}\left\{\frac{v_{w}+a \omega}{v_{u}}\right\}
$$

During the motion of vehicle the lateral forces exerted by the tires on the vehicle are given by [35] by following the approach given in [34]:

$$
\begin{aligned}
& \dot{v}_{u}=G_{u} F_{u}+G_{s} F_{s}+G_{p} F_{p}, \\
& v_{w}=\frac{v_{u} \cdot b \cdot \tan \delta}{L} \\
& \dot{\theta}=\omega=\left(v_{u} / L\right) \times \tan \delta \\
& \dot{x}=v_{u} \cos \theta-v_{w} \sin \theta \\
& \dot{y}=v_{u} \sin \theta+v_{w} \cos \theta
\end{aligned}
$$

where,

$$
G_{p}=\left[m+\left(\frac{\tan \delta}{L}\right)^{2}\left(m b^{2}+j\right)\right]^{-1}
$$




$$
\begin{aligned}
G_{u} & =G_{p}\left(\begin{array}{ll}
\frac{1}{\cos \delta} & 1
\end{array}\right) \\
G_{s} & =-\frac{m b^{2}+j}{L \cos ^{2} \delta} \omega G_{p}
\end{aligned}
$$

Since, here $\mathrm{w}$ have considered $v_{w}=0$ and the force $F_{u}=0$, the expected value of longitudinal perturbation.

The equation (5.20), (5.23), (5.24) will reduce to:

$$
\begin{aligned}
& \dot{v}_{u}=G_{s} F_{s}+G_{p} F_{p} \\
& \dot{x}=v_{u} \cos \theta \\
& \dot{y}=v_{u} \sin \theta
\end{aligned}
$$

All above equation forms the dynamic model for car-like mobile robot.

\subsection{Path-tracking controllers design}

For path tracking problem is the problem of determining the propulsion and steering inputs required for the vehicle to follow the assigned path with given velocity. The planner is provided with required configuration $q_{d}(c)$, which is a smooth vector function given as:

$$
q_{d}(c)=\left[\begin{array}{l}
x_{d}(c) \\
y_{d}(c) \\
\theta_{d}(c) \\
\delta_{d}(c)
\end{array}\right]
$$


Where, $c \in R$ and $\left(x_{d}(c), y_{d}(c)\right)$ is the position which, the reference point on the vehicle is required to have at the point of path defined by $c, \theta_{d}(c)$ and $\delta_{d}(c)$ are the orientations that the vehicle is required to achieve at $c$. The velocity profile is a smooth function with which the vehicle has to follow the path. Using the above stated required configurations, the offsets that are generated which are the difference between the current states and required states are given as:

$$
\begin{array}{ll}
v_{o s}=v_{u}-v_{u d}, & \text { velocity offset } \\
\theta_{o s}=\theta-\theta_{d}, & \text { orientation offset } \\
\delta_{o s}=\delta-\delta_{d}, & \text { steering angle offset. } \\
l_{o s}=-\left(x-x_{d}\right) \sin \theta_{d}+\left(y-y_{d}\right) \cos \theta_{d}, \text { lateral position offset }
\end{array}
$$

If the path in the workspace is straight line or a circular path with constant desired tracking velocity and the path-tracking offsets are kept sufficiently small, then pathtracking for a car-like robot can be obtained by combination of two-invariant controllers, that is, to have:

$$
\lim _{t \rightarrow 0}\left[\begin{array}{l}
v_{o s} \\
\theta_{o s} \\
l_{o s} \\
\delta_{o s}
\end{array}\right]=0
$$

Offset $\theta_{o s}$ gives the measure of orientation error relative to path, $l_{o s}$ is the measure of lateral position error and the amount by which the vehicle is moving far from the trajectory. First controller is steering controller which provides the steering action by 
generating steering acceleration $F_{s}$ and propulsion controller or speed controller which maintains vehicle to move with desired velocity by generating propulsion force $F_{p}$.

This above definition is known as Path tracking or path following problem.

The steering controller is given as [35]:

$$
\mathbf{F}_{s}=-\mathbf{K}_{s}\left[\begin{array}{lll}
\theta_{o s} & l_{o s} & \delta_{o s}
\end{array}\right]^{T}
$$

Where, $\mathbf{K}$ is steering controller gain.

Now, under the slippage free motion, path tracking is equivalent to stabilizing the dynamic system:

$$
\begin{aligned}
& \dot{\theta}_{o s}=\frac{\delta_{o s} \cdot v_{u d}}{L \cdot \cos ^{2} \delta_{d}} \\
& \dot{l}_{o s}=v_{d} \theta_{o s}+b \dot{\theta}_{o s} \\
& \dot{\delta}_{o s}=\mathbf{F}_{s}
\end{aligned}
$$

Where, $v_{d}$ is desired velocity of the vehicle and is given as [35]:

$$
v_{d}=v_{u d} \sqrt{1+\left(\frac{b \tan \delta}{L}\right)^{2}}
$$

Using the dynamics properties of the offsets as shown above the controller design can be considered as equivalent to stabilizing the system:

$$
\dot{\mathbf{x}}=\mathbf{A x}+\mathbf{B u}
$$


Where, $\quad \mathbf{A}=\left(\begin{array}{ccc}0 & 0 & \frac{v_{u d}}{l \cos ^{2} \delta_{d}} \\ v_{d} & 0 & \frac{b v_{u d}}{l \cos ^{2} \delta_{d}} \\ 0 & 0 & 0\end{array}\right) \quad$ and $\quad \mathbf{B}=\left(\begin{array}{l}0 \\ 0 \\ 1\end{array}\right)$

Since the matrix $\boldsymbol{A}$ in our work depends on value of desired steering angle, which is variable at every time step, $\boldsymbol{A}$ is dynamic.

The gains $\mathbf{K}_{s}$ are selected such that to stabilize the system $\dot{x}=\left(\mathbf{A x}-\mathbf{B K} \mathbf{s}_{s}\right) \mathbf{x}$ by using feed-back control law i.e. $\mathbf{u}=-\mathbf{K}_{S} \cdot \mathbf{x}$ and the states vector for such system is $\mathbf{x}=\left[\begin{array}{lll}\theta_{o s} & l_{o s} & \delta_{o s}\end{array}\right]^{T}$

Here, suitable gains are selected using Linear Quadratic Regulator (LQR). The LQR problem formulation facilitates for choosing variety of gains by carrying out trial and error method. $L Q R$ is the problem of finding gains that minimizes the mean square error or minimizing the cost function I as:

$$
\mathbf{I}=\int_{0}^{\infty}\left(\mathbf{x}^{T} \mathbf{Q} \mathbf{x}+\mathbf{u}^{T} \mathbf{R} \mathbf{u}\right) d t
$$

Selection of $\mathbf{Q}$ and $\mathbf{R}$ depends on designer and are selected based on required performance and it's the ratio $(\mathbf{Q} / \mathbf{R})$ which is responsible for a particular performance of controller. The gains are generated for particular combination of $\mathbf{Q}$ and $\mathbf{R}$ by using famous Riccatti equation which can be written as:

$$
\mathbf{P A}+\mathbf{A}^{T} \mathbf{P}-\mathbf{P B Q} \mathbf{Q}^{-1} \mathbf{B}^{T} \mathbf{P}+\mathbf{R}=0
$$

By solving this equation we get $\mathbf{P}$ and the gains are obtained as: 


$$
\mathbf{K}=\mathbf{Q}^{-1} \mathbf{B}^{T} \mathbf{P}
$$

MATLAB has a facility to solve this equations directly using function lqr.m. Using the gains generated using LQR, the minimization of offsets is achieved by $\mathbf{K}_{s}$, such that:

$$
\mathbf{F}_{s}=-\mathbf{K}_{s}\left[\begin{array}{lll}
\theta_{o s} & l_{o s} & \delta_{o s}
\end{array}\right]^{T}=-K_{s_{1}} \theta_{o s}-K_{s_{2}} l_{o s}-K_{s_{3}} \delta_{o s}
$$

To consider all conditions with different steering angles are relatively difficult and complex. The velocity controller is given as [35]:

$$
F_{p}=F_{p_{1}}+F_{p_{2}}
$$

Where, $F_{p_{1}}=-G_{p}^{-1} G_{s} F_{s}$ and $F_{p_{2}}=-G_{p}^{-1}\left[K_{p_{1}} v_{o s}+K_{p_{2}} \int v_{o s} d t\right]$

Selection of gains $K p_{1}$ and $K p_{2}$ is based on indirect Lyapunov's stability criterion and

$$
K p_{1}=-\left(k_{1}+k_{2}\right) \text { and } K p_{2}=k_{1} \cdot k_{2}
$$

The equation (5.51) is simply a PI controller, whose gains are based on selections of closed loop poles of the dynamic characteristic equation which is (5.51). Also the performance of controller for particular set of gains selected is taken into account. Again, the dynamics equations as given by (5.23), has the property such that:

$$
\ddot{v}_{o s}-\left(k_{1}+k_{2}\right) \dot{v}_{o s}+k_{1} k_{2} v_{o s}=0
$$

Where, $k_{1}$ and $k_{2}$ are the roots of characteristic equation (5.54) and are poles of system. The locations of these poles are decided so that controller is stable. An obvious selection would be the poles on the left side of s-half plane that always brings system to equilibrium regardless of value of initial conditions and hence system is asymptotically stable. In this work, the poles for characteristic equation (5.54) is selected as $k_{1}=5$ and $k_{2}=0.5$ such that both the poles are on left half of s-plane with damping coefficient 
$\zeta=1.73$ and hence system is over damped. The characteristic equation will be

$$
\ddot{v}_{o s}-(5+0.5) \dot{v}_{o s}+(5 \times 0.5) v_{o s}=0
$$

And the step response will be:

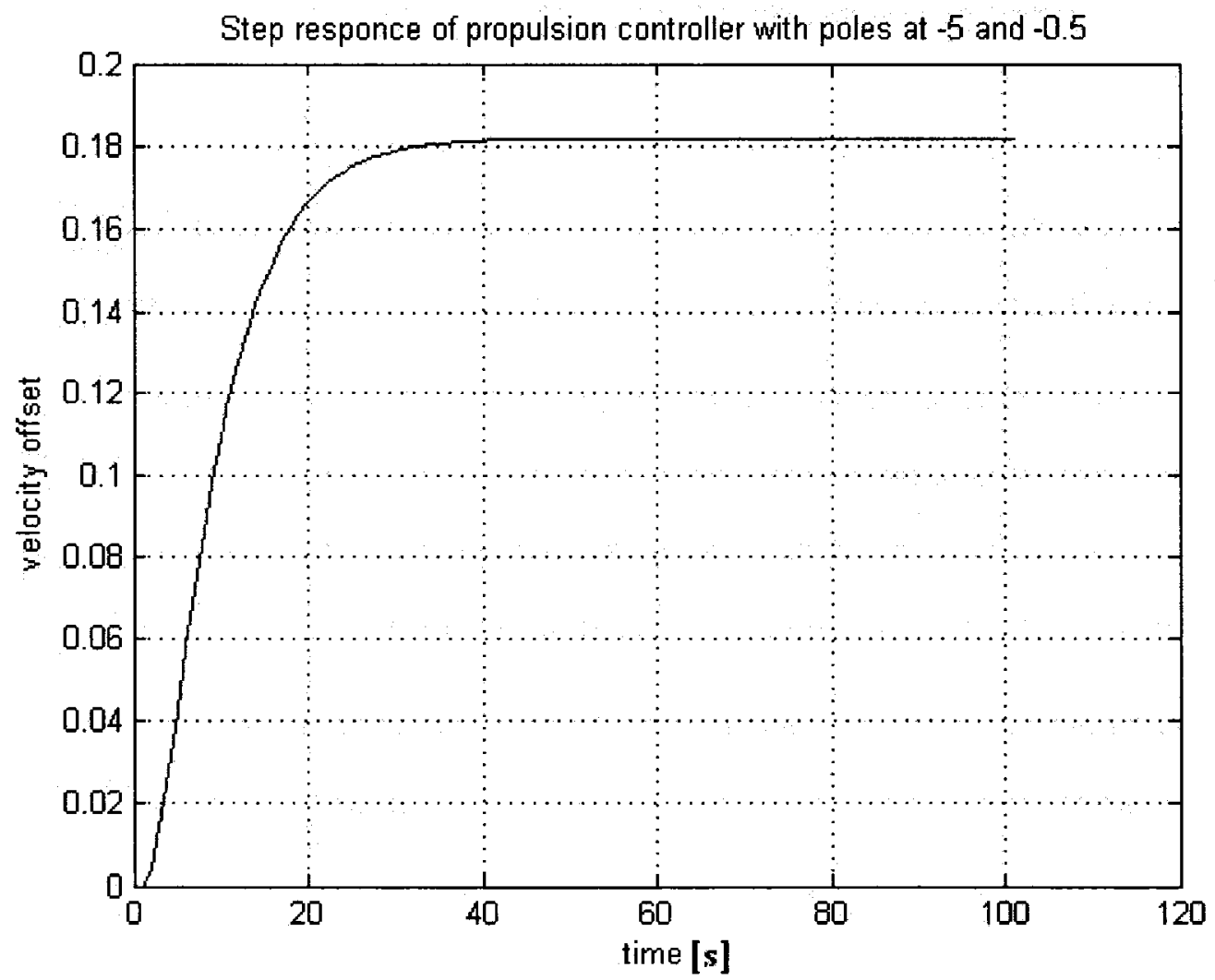

Figure 5.2: Step response of propulsion controller

These gains are substituted in the equation (5.52) and hence $F_{p}$ by substituting values of $F_{p_{1}}$ and $F_{p_{2}}$ in equation (5.51). As we see, from equation $(5.52), F_{p}$ is directly proportional to $\mathbf{F}_{s}$ and since $\mathbf{F}_{s}$ is bounded, $F_{p_{1}}$ is also bounded. The block diagram for path-tracking steering controller is as shown in figure below: 


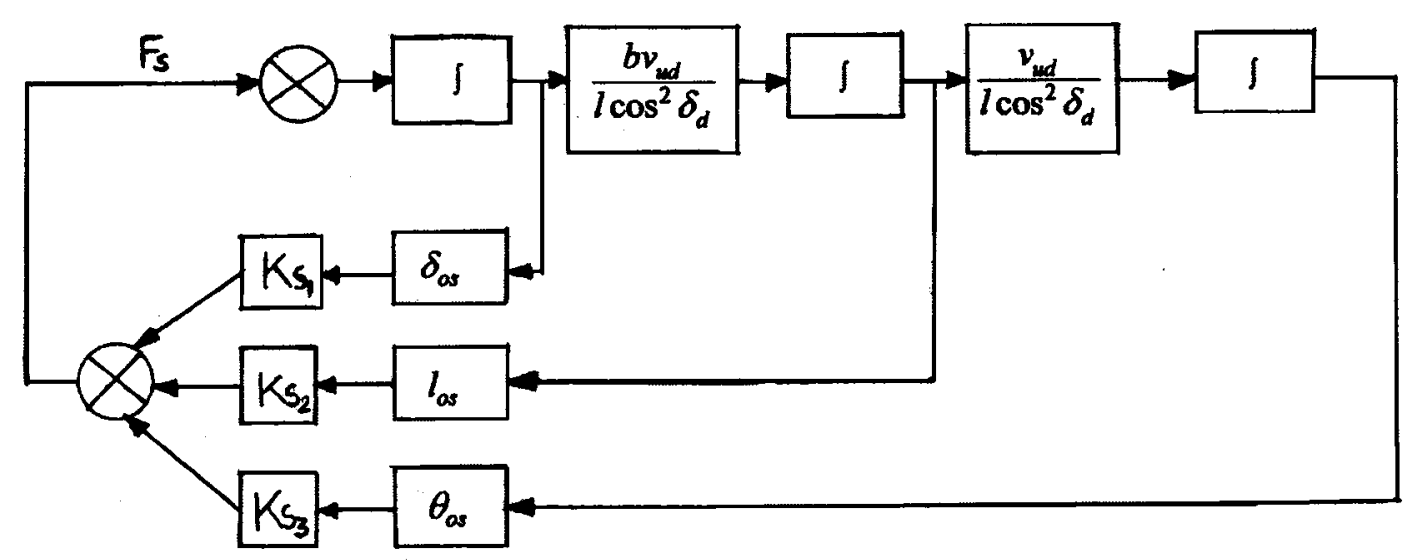

Figure 5.3: Block diagram of steering controller

\section{Selection of parameters for controllers}

Consider, for instance, the desired steering angle required for next step is 10 degrees, length 1 and wheel base $b$ of robot is 1.5 meters and 1 meter respectively as in this work and if the desired longitudinal velocity is 1 meter per second then above $\mathbf{A}$ matrix is given as:

$$
\mathbf{A}=\left(\begin{array}{ccc}
0 & 0 & 0.6873 \\
1 & 0 & 0.6873 \\
0 & 0 & 0
\end{array}\right) \text { and } \mathbf{B}=\left(\begin{array}{l}
0 \\
0 \\
1
\end{array}\right)
$$

Using this matrix configuration simulation is carried out for different parameters of the controller and the one which gives better results are selected in this work. To see the open loop response of the model as given by (5.55), let us give the simple step input by providing some initial error conditions for all three errors. Consider error initial conditions as zero, the input $u=10$, rate of change of steering angle for period of $10 \mathrm{~s}$ with the sampling time of $0.1 \mathrm{~s}$. The speed of vehicle is 1 meter per second and the initial 
steering angle is kept at 25 degrees. The open loop response for the error model is as shown in figure below:

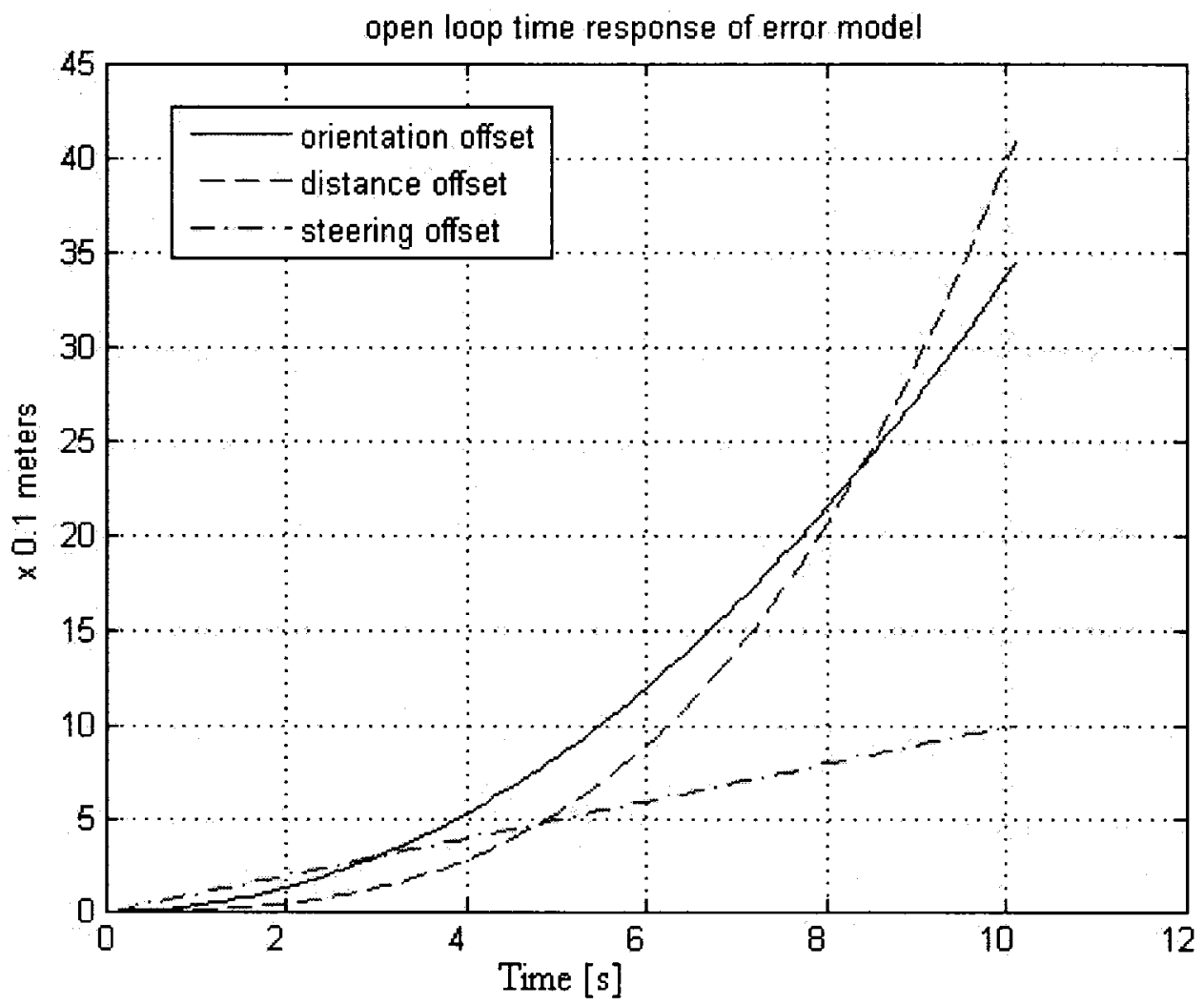

Figure 5.4: Open loop time response of error model.

It can be noticed that for this particular input the rate at which error tends to increase. Maximum orientation error was found to be 35 degrees, maximum length offset is found to be 41 meters and maximum steering angle offset is found to be 10 degrees. Although the relationship between these response signals will change for different time of operation, they will be similar and will be scaled in magnitude. The feedback gains may be chosen from carrying out series of experiments or simulations. In order to choose the set of control gains for use of control law as given by equation $\mathbf{u}=-\mathbf{K}{ }_{S} . \mathbf{x}$, the characteristics of open loop pole 
is considered. $\mathbf{Q}$ and $\mathbf{R}$ matrices are parameters for $\mathrm{LQR}$ for steering controller and closed loop poles for velocity controller. Since, in our work, the state variables are three, the size of $\mathbf{Q}$ matrix is (3×3) and the control signal is steering velocity for steering controller, $\mathbf{R}$ is matrix with only one element. Here, we have selected matrix $\mathbf{Q}$ as diagonal matrix with all elements of diagonal are same but varies with some multiple.

$$
\mathbf{Q}=C_{1} \times\left(\begin{array}{ccc}
1 & 0 & 0 \\
0 & 1 & 0 \\
0 & 0 & 0.1
\end{array}\right) \text { and } \mathbf{R}=C_{2}
$$

Different combinations of $C_{1}$ and $C_{2}$ is as shown in table below and the value of these constants are decided based on response of each combination. State feedback methods could also be used to achieve the control of offsets but the main purpose of using LQR is to have different weights to different states. Here we give more weights to orientation and length offsets than steering offsets. 


\begin{tabular}{|c|c|c|c|c|c|c|c|c|c|}
\hline Serial & $C_{1}$ & $C_{2}$ & $\left(C_{1} / C_{2}\right.$ & $\mathrm{K}_{\mathrm{s} 1}$ & $\mathrm{~K}_{\mathrm{s} 2}$ & $\mathrm{~K}_{\mathrm{s} 3}$ & $e_{1}$ & $e_{2}$ & $e_{3}$ \\
\hline 1 & 1 & 1 & 1 & 1.9178 & 1 & 2,2386 & $\begin{array}{r}-0.71 \\
+0.58 \mathrm{i}\end{array}$ & $\begin{array}{l}-0.71 \\
-0.58 \mathrm{i}\end{array}$ & -0.81 \\
\hline 2 & 1 & 10 & 0.1 & 0.8718 & 0.3162 & 1.3165 & $\begin{array}{r}-0.36 \\
+0.49 \mathrm{i}\end{array}$ & $\begin{array}{r}-0.36 \\
+0.49 \mathrm{i}\end{array}$ & -0.55 \\
\hline 3 & 1 & 50 & 0.02 & 0.5192 & 0.1414 & 0.9634 & $\begin{array}{r}-0.26 \\
+0.39 \mathrm{i}\end{array}$ & $\begin{array}{l}-0.26 \\
-0.39 i\end{array}$ & -0.43 \\
\hline 4 & 20 & 1 & 20 & 6.3364 & 4.4721 & 5.9042 & $\begin{array}{r}-0.77 \\
+0.33 \mathrm{i}\end{array}$ & $\begin{array}{l}-0.77 \\
-0.33 i\end{array}$ & -4.36 \\
\hline 5 & 10 & 1 & 10 & 1.9223 & 3.1623 & 4.1219 & $\begin{array}{r}-0.78 \\
+0.33 \mathrm{i}\end{array}$ & $\begin{array}{l}-0.78 \\
-0.33 i\end{array}$ & -3 \\
\hline 6 & 20 & 10 & 2 & 2.4790 & 1.4142 & 2.7115 & $\begin{array}{r}-0.84 \\
+0.48 \mathrm{i}\end{array}$ & $\begin{array}{l}-0.84 \\
-0.48 i\end{array}$ & -1.02 \\
\hline 7 & 5 & 10 & 0.5 & 1.4990 & 0.7071 & 1.8796 & $\begin{array}{r}-0.58 \\
+0.58 \mathrm{i}\end{array}$ & $\begin{array}{l}-0.58 \\
-0.58 \mathrm{i}\end{array}$ & -0.70 \\
\hline 8 & 0.1 & 10 & 0.01 & 0.4167 & 0.1 & 0.8487 & $\begin{array}{l}-0.22 \\
+35 i\end{array}$ & $\begin{array}{l}-0.22 \\
-35 \mathrm{i}\end{array}$ & -0.39 \\
\hline
\end{tabular}

Table 5.1: LQR controller parameters

Performance results of such controller with selection of different values of $\mathbf{Q}$ and $\mathbf{R}$ matrices for LQR is as shown in chapter 7. To observe the behavior of controller with different values of $\mathbf{Q}$ and $\mathbf{R}$, we have selected a common trajectory. As shown in table, 
eight cases were considered for different values of $\mathbf{Q}$ while $\mathbf{R}$. From results we will see that as the ratio $\left(C_{1} / C_{2}\right)$ increases the gains are also higher and the eigen values corresponding to higher ratio shifts more towards left side of s-plane. This results in more stable performance but the rise time decreases. On basis of these, the ratio of 10 is fixed in this work with the values of $C_{1}=10$ and $C_{2}=1$. The eigen valued corresponding to this ratio are $-0.78+0.33 i,-0.78+0.33 i,-0.1098$. Since all eigen values are negative, response will be stable though with transient response. Path tracking distance and orientation offsets with this combination are shown is figures for straight line trajectory as below:

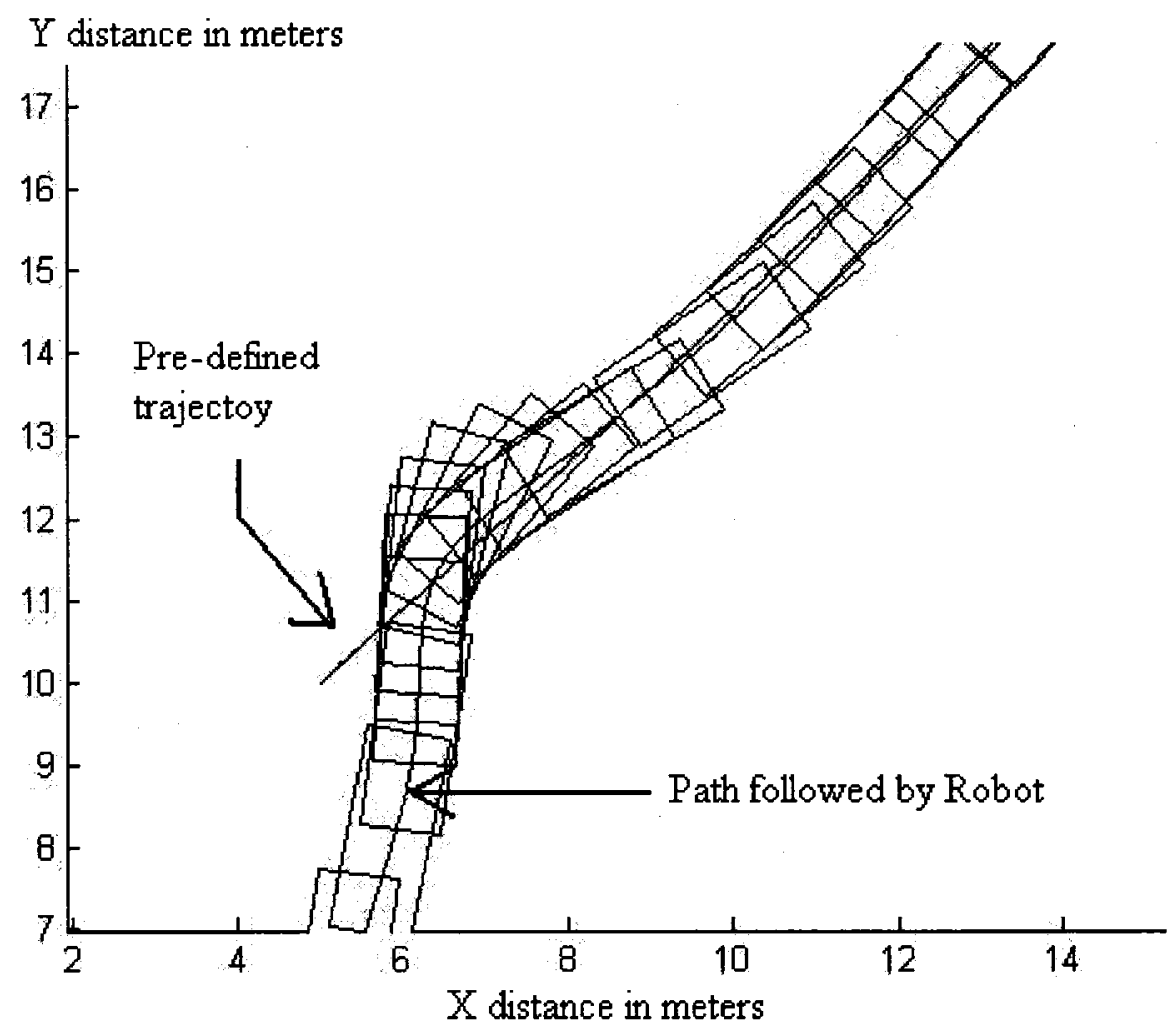

Figure 5.5: Path tracking on straight path with $C_{1}=10$ and $C_{2}=1$. 


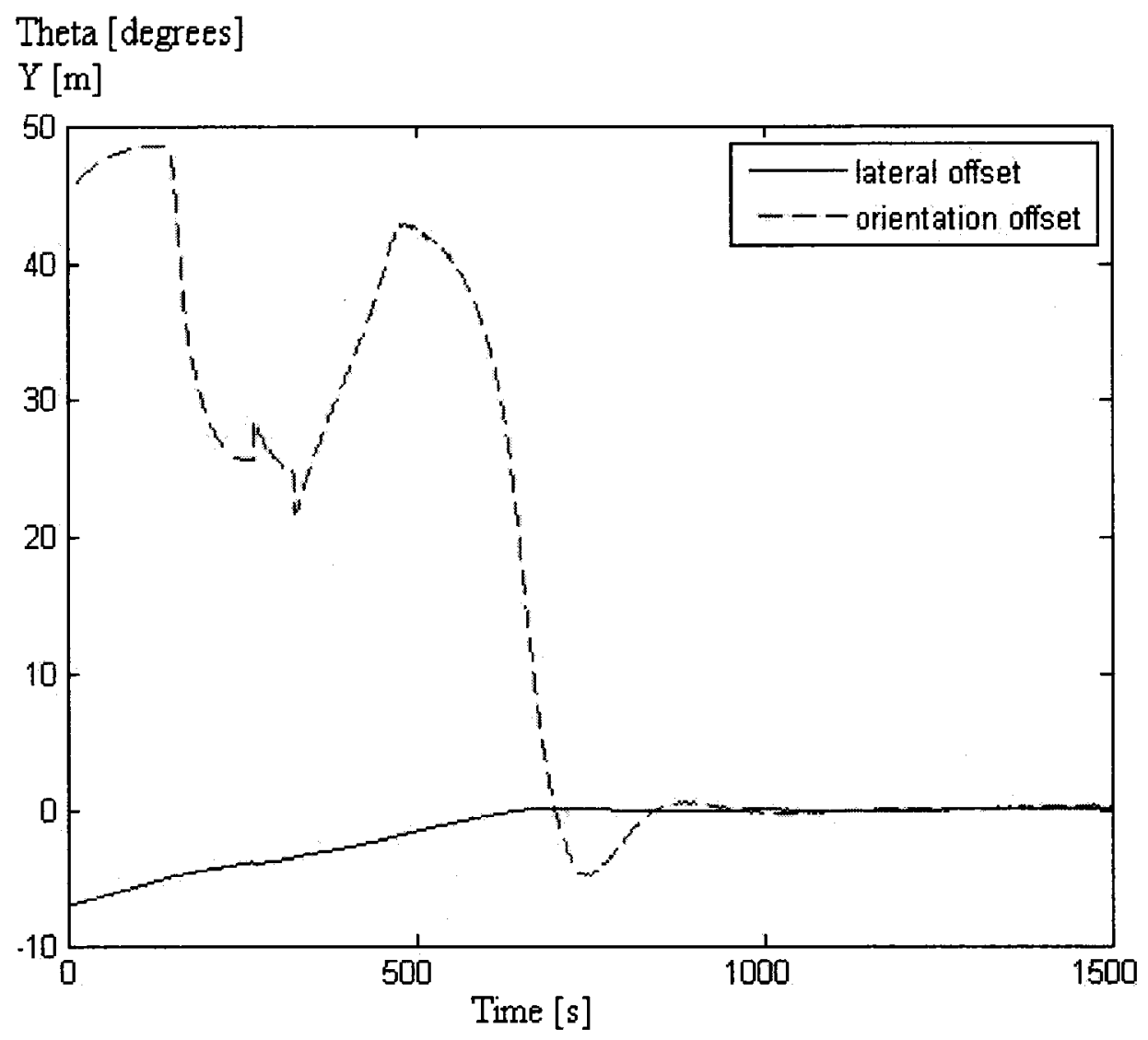

Figure 5.6: Distance offset and orientation offset for $C_{1}=10$ and $C_{2}=1$.

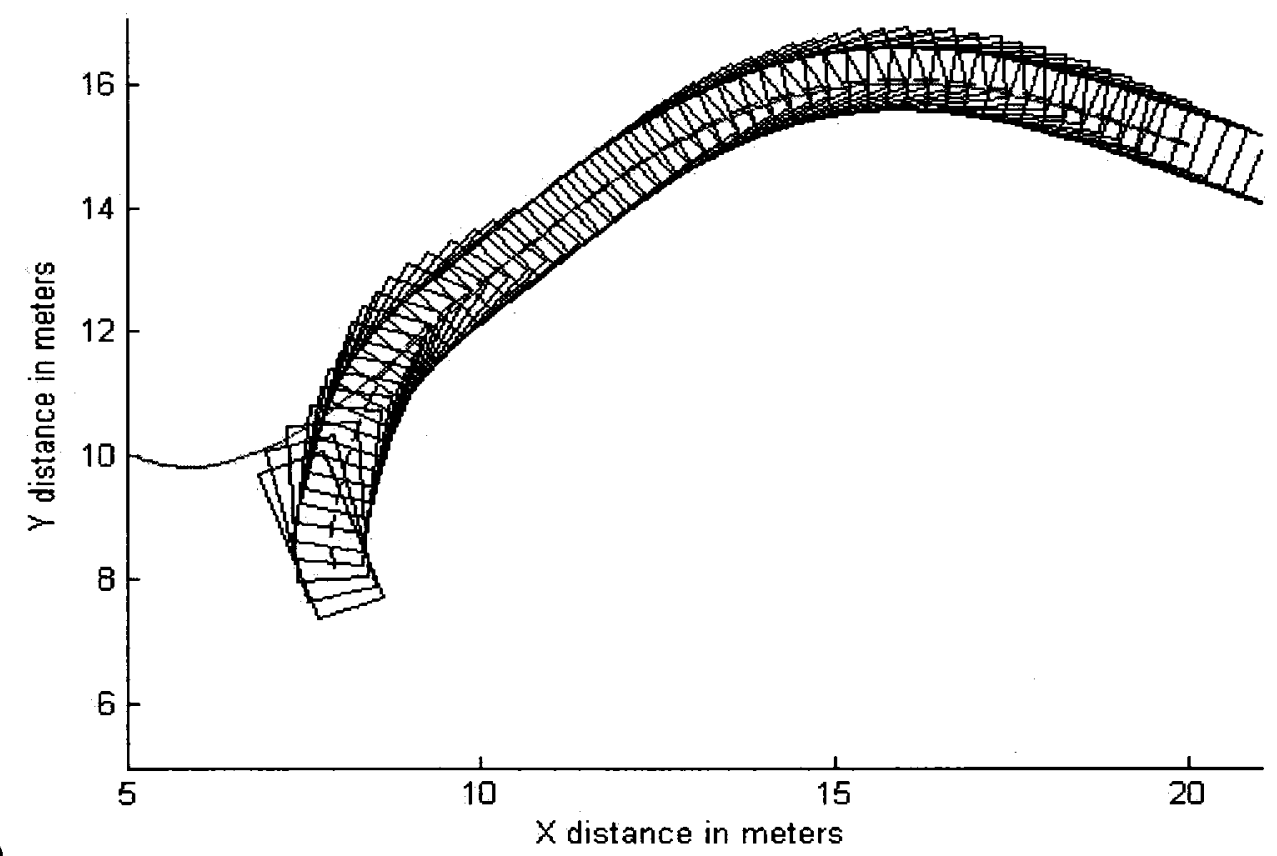

(a) 


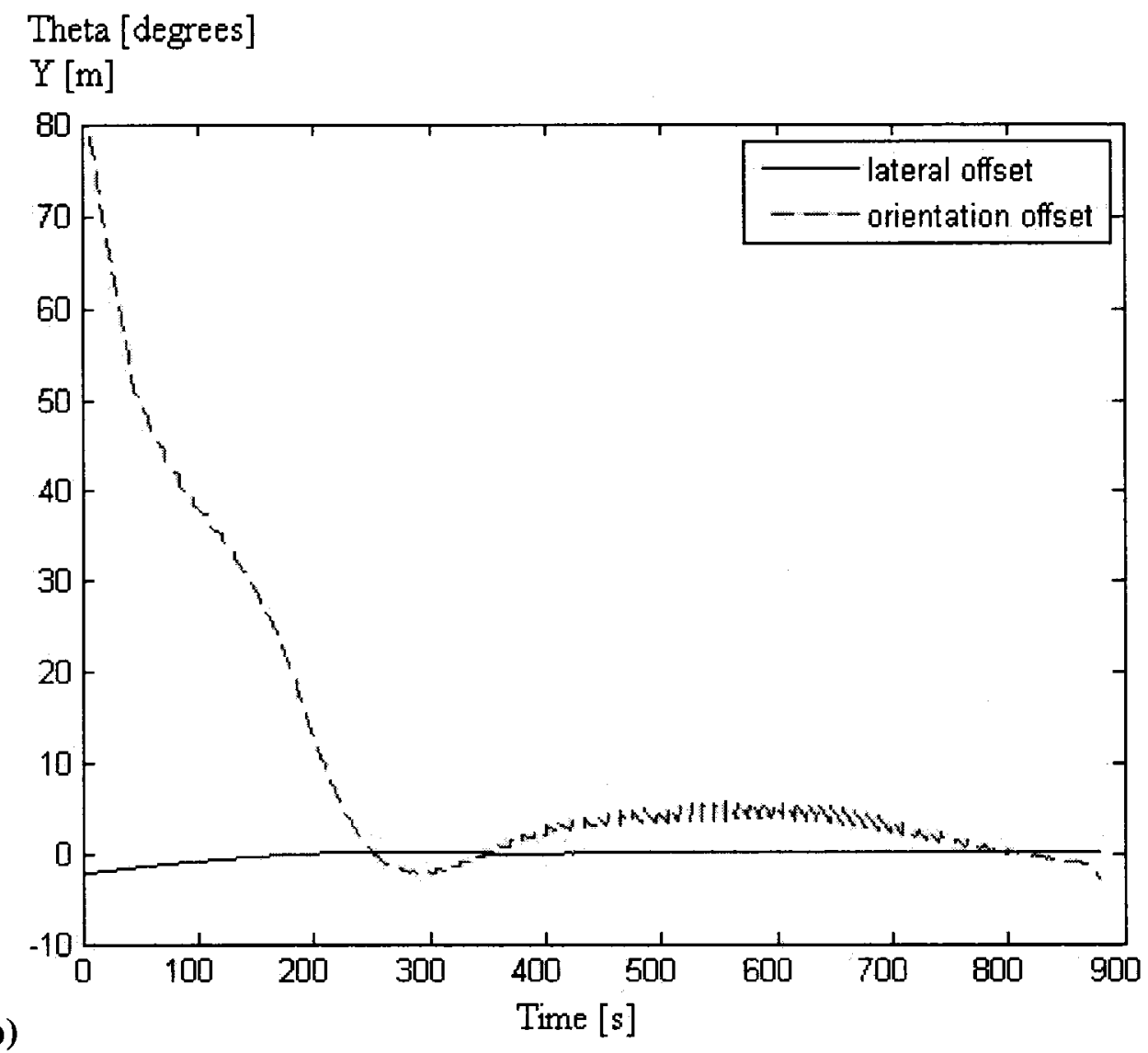

Figure 5.7: Path tracking on curved path with $C_{1}=10$ and $C_{2}=1$. 


\section{Chapter 6: Collision avoidance algorithm}

\subsection{Introduction and literature review for collision avoidance}

The goal of this work is navigation of mobile robots with obstacle avoidance for car-like robot when it is moving on or away from the trajectory by detecting the obstacle within the certain distance from the robot. During obstacle avoidance it also keeps into the consideration the predetermined trajectory so as to remain close as much as possible to trajectory and also come back to trajectory after obstacle avoidance. By adopting this, the robot can reach to its designed trajectory and hence destination consuming less time. To carry out this, the robot should first know its location and the knowledge of the surrounding environment as described in chapters 3 and 4. Path tracking of robot on trajectory is discussed and the results are shown in chapter 5. This chapter deals with the step by step description of collision avoidance algorithm proposed in this work.

Robot navigation problems, in general may be classified as global navigation or local navigation, depending upon the environment surrounding the robot. In case of global navigation, the environment of the robot is known previously and the obstacles are identified. The roadmaps, as discussed in chapter 4 , are constructed and the optimal path is searched that can avoid the obstacle. In case of local navigation, the environment is not known or partially known. The sensors are used to detect the obstacles in the robots surroundings and the collision avoidance algorithm must be incorporated into the robots program so as to generate collision free path online. Some obstacle avoidance techniques include potential field methods [25], adaptive navigation of mobile robots with obstacle 
avoidance [36], VHP methods [8], VFH methods [9], and bug algorithm [25]. Let us discuss in brief potential field method and bug algorithm as below:

\subsubsection{Potential field}

A potential function is differentiable real valued function defined as function $U: R \rightarrow R$. It is energy hence the gradient of potential is nothing but force. Gradient is a vector that points in the direction that locally maximally increases $U$ and the vector field assigns a gradient vector to each point on a manifold. In potential field the robot is considered as a particle moving in a gradient field. The gradients are the force that acts on a robot which is considered as positively charged particles that attracts towards negatively charged goal point. The obstacles in the field also acts as positively charged, hence the gradient forces will generate repulsive force to keep robot away from the obstacle. The combination of these attractive and repulsive forces "hopefully" directs the robot from the starting point to goal point while avoiding obstacles. In this, the robot is directed towards low valued potential to higher valued potential. Following such path is known as "gradient descent" [5]

$$
\dot{q}(t)=-\nabla U(q(t))
$$

When the gradient reduces to zero, the robot stops the motion at that spot. This point is known as critical point. The three types of critical points are maximum, minimum and saddle point. For gradient descent methods, the robot usually starts at local maximum and terminates at local minimum. When robot starts from local maximum, it gets stuck there and can be freed into the field by small perturbations allowing the gradient vector field to induce motion onto the robot. Whereas, local minimum are stable as when any 
perturbations given at minimum, robot will return to minimum only. Hence, the only point in the field where robot terminates is local minimum. Because of this reason this method has a drawback that if the point at local minimum is not the goal point then it will terminate motion assuming it as a goal but in fact, its not. Two classes of approaches address this problem [37]. First class augments the potential field with search-based planner, while second defines potential field with local minimum, also known as navigation function. Both these approaches require prior and complete knowledge of configuration space.

\subsubsection{Bug algorithm}

Bugs algorithms [25] are among the oldest sensor-based planners and assume the robot as a point operating in plane with sensors that detect the obstacles. The sensors may be touch sensors or with distance measuring capabilities. With later, bug algorithm is then tangent bug algorithm [25]. These algorithms use sensors observations to find shortest paths to goals and are very simple and straightforward to implement, that require two behaviors: straight line and boundary following. In this the robot move in direction of goal until it encounters an obstacle and circumnavigates the obstacle until it finds the path which is shortest feasible. It's based on "common sense" idea of moving towards goal and going around obstacle. The point where robot detects obstacle is known as hit point. It will then circumnavigate the obstacle till it reaches hit point and then searches for the point making a shortest distance with goal. If the line joining a selected point and a goal intersects with edge of obstacle, next closest point is chosen. The point where robot leaves from obstacle is 
called leave point. The basic difference between Bug1 and Bug2 is the method of selection of leave point. The algorithm of this method is given in detail in [25].

\subsection{Collision avoidance design}

Some of the most popular collision avoidance methods are introduced in previous section. Car-like robots have kinematic constraints as well as its dynamics must also be taken into consideration while designing the navigation system. This work focuses on local navigation problem considering dynamics and kinematics constraints of Car-like mobile robots which are already discussed in chapter 2 . Information regarding initial robot location, goal location and original trajectory is known. With the beginning of task, the distance from the original trajectory is determined and if robot if far from trajectory, the point making a minimum distance on trajectory should be selected as "target" and necessary steering angle is determined. During this process, there is possibility of detecting an obstacle which is further avoided by switching to collision avoidance algorithm in which the robot circumnavigates around the obstacle. Collision avoidance algorithm determines the steering angle taking into consideration goal point, number of obstacles and trajectory locations so that robot does not deviate much away from the trajectory and hence reducing time to reach goal. If the robot is moving on trajectory, it should consider only that obstacle that might come on its way or near the trajectory, thereby reducing overall processing time at each time step. Laser range finder is provided at the front of robot whose range (or distance) and angular reading are used for mapping of the environment using occupancy grid method as discussed in chapter 4 . The direction 
of "target" point and hence steering angle is calculated. The method for calculating steering angle given target point is discussed later in this chapter. Desired steering is used to control motion using simple PD controller. The advantage of this method is that it is simple to simulate and less information regarding environment is required for local path planning.

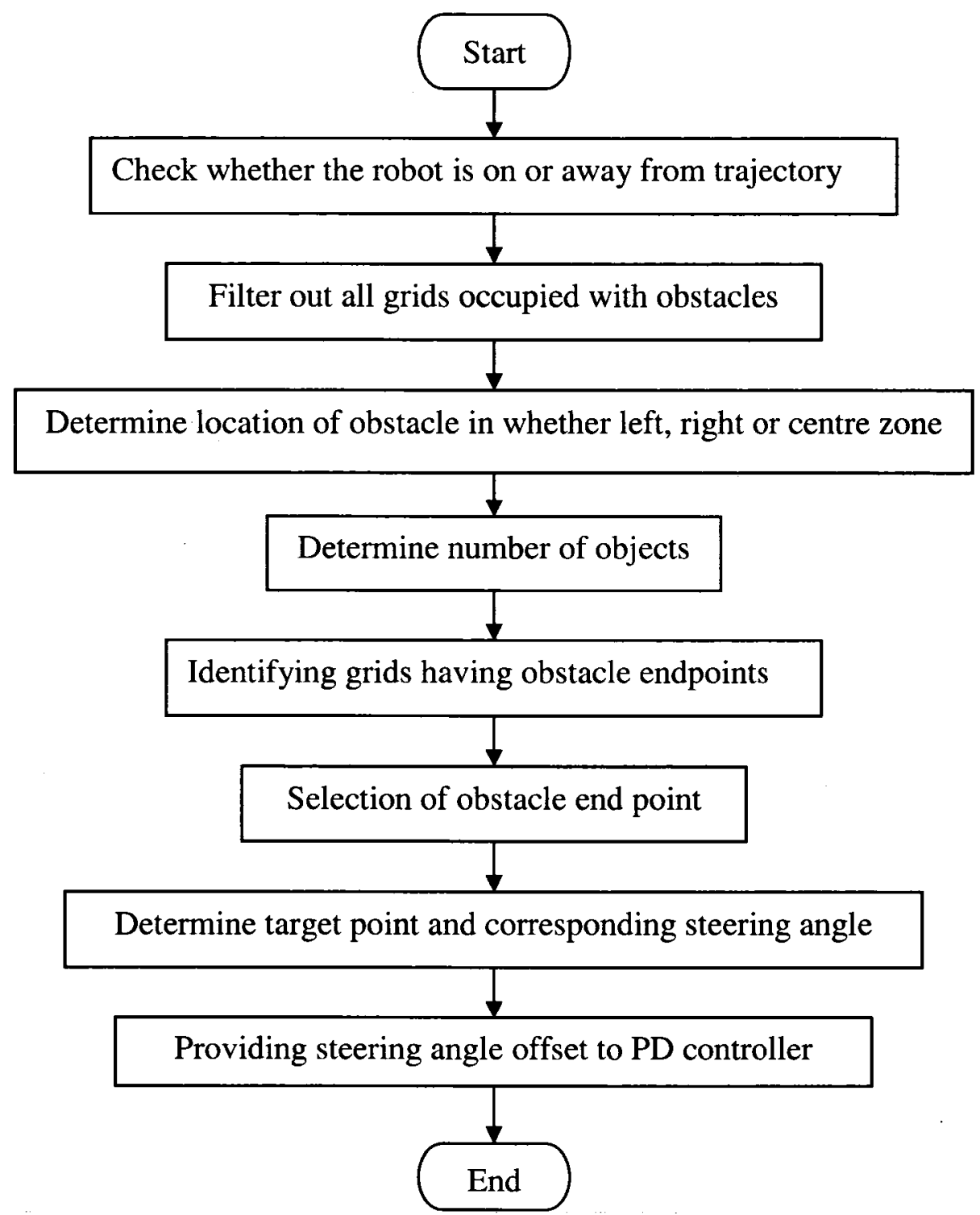

Figure 6.1: Structure of collision avoidance 


\section{Assumptions:}

1) Robot moves in forward direction with steering range of $+/-45$ degrees.

2) Its turning radius of curvature is limited to $\rho_{\min }$.

3) Moves with constant velocity of 1 meter per second.

4) Maximum range of collision detection is limited to 20 meters.

5) All the obstacles are modeled as convex polygons.

\subsubsection{Collision detection}

Spatial representation of local surrounding environment in the form of occupancy grid map is generated using laser range sensor reading after filtering out noise using EKF. Out of all those grids, the grid points that are in collision zone are filtered out first and only these points are taken into consideration for obstacles detection. The collision zone is defined as the conical region in front of robot within the maximum range of 20 meters and makes an angle of $+/-65$ degrees with respect to robots longitudinal axis. The selection of maximum range is done arbitrarily and can be changed based on application. Further, processing of grids is carried out to identify the occupied grids and to detect the number of obstacle in collision zone along with their end points as represented by grid points. 


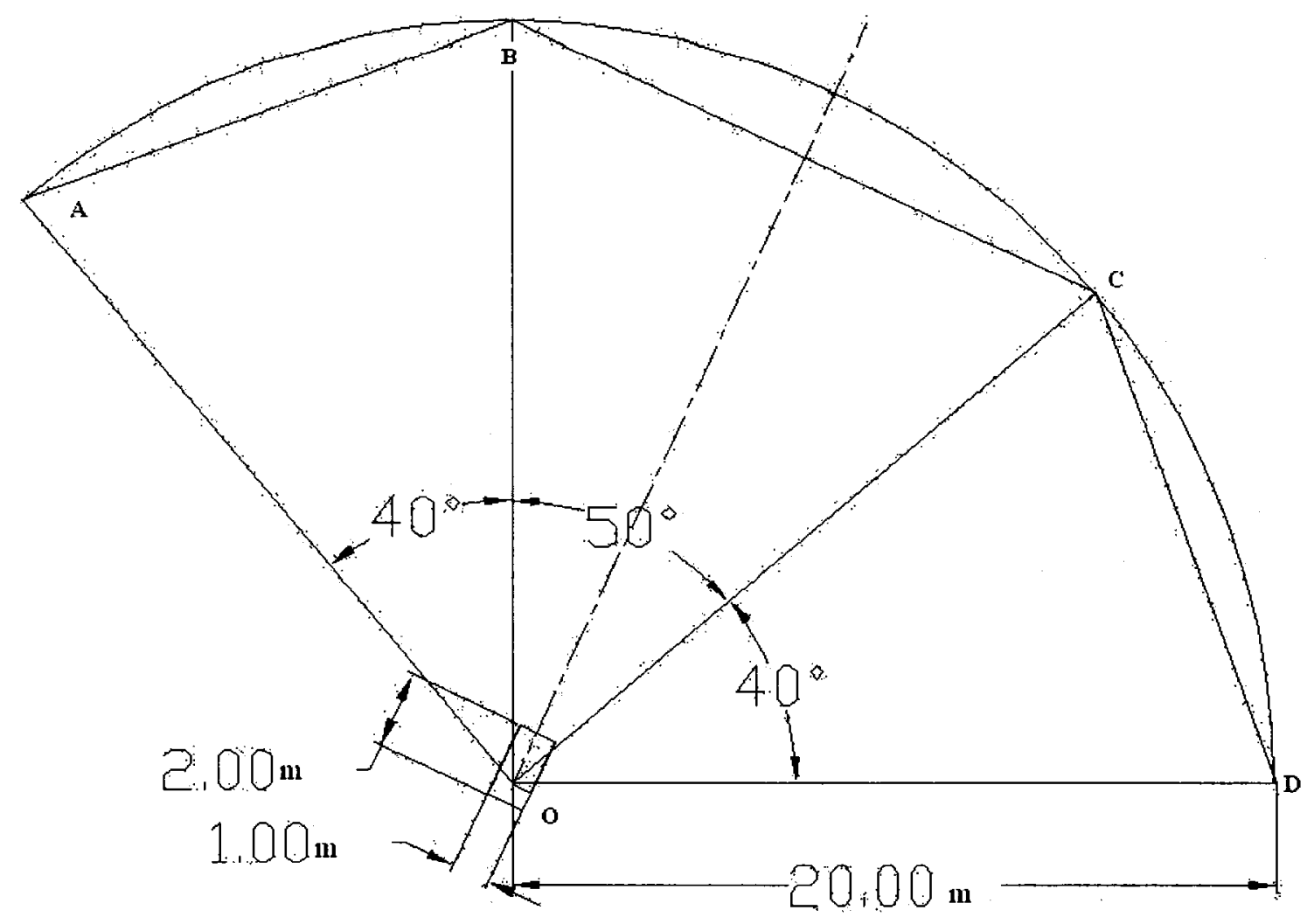

Figure 6.2: Collision zone (in meters)

During detection if the collision is found, collision detection status is set to 1 or else it is unchanged with the initial value of -1 . For simplicity, the total collision zone is divided into three zones that are front zone, left zone and right zone as shown above. Based on these zones, collision avoidance problem can be classified in three different cases. These are one detection, two detection and three detection cases. Each of theses three cases are handled in different manner. Further, from all the three detections, some cases are handled differently if the detected obstacle is one or more than one. Further robot checks that whether robot is on the trajectory or away from the trajectory and again both this cases are handled in different manner. 
All three cases with one or more objects and also with robot on or away from trajectory are explained all together for each case.

\section{1) One detection case}

This is due to presence of an obstacle in only one of three collision zones. There are three possibilities: obstacle on left, right or in front zone as shown below:

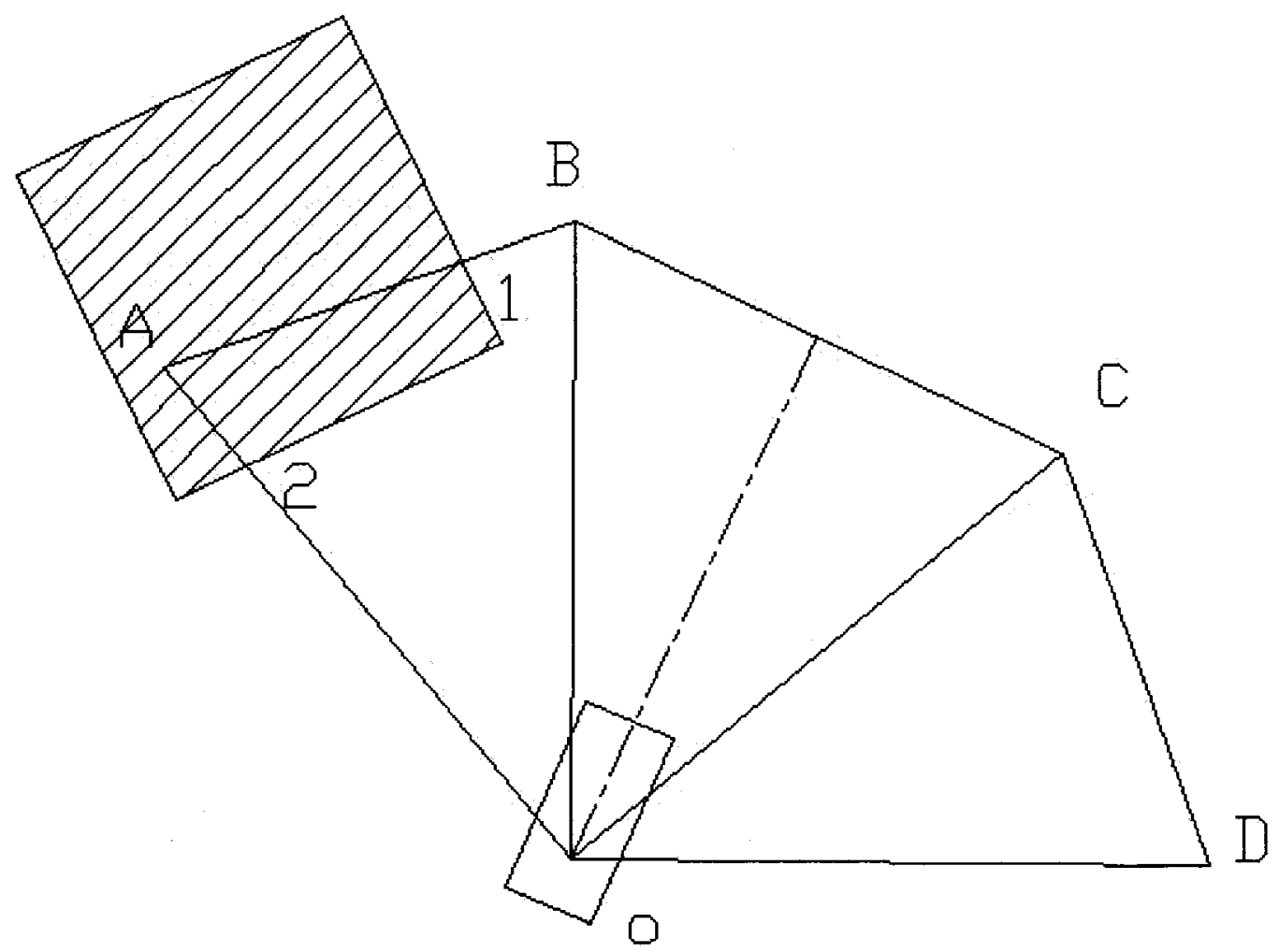

(a) 


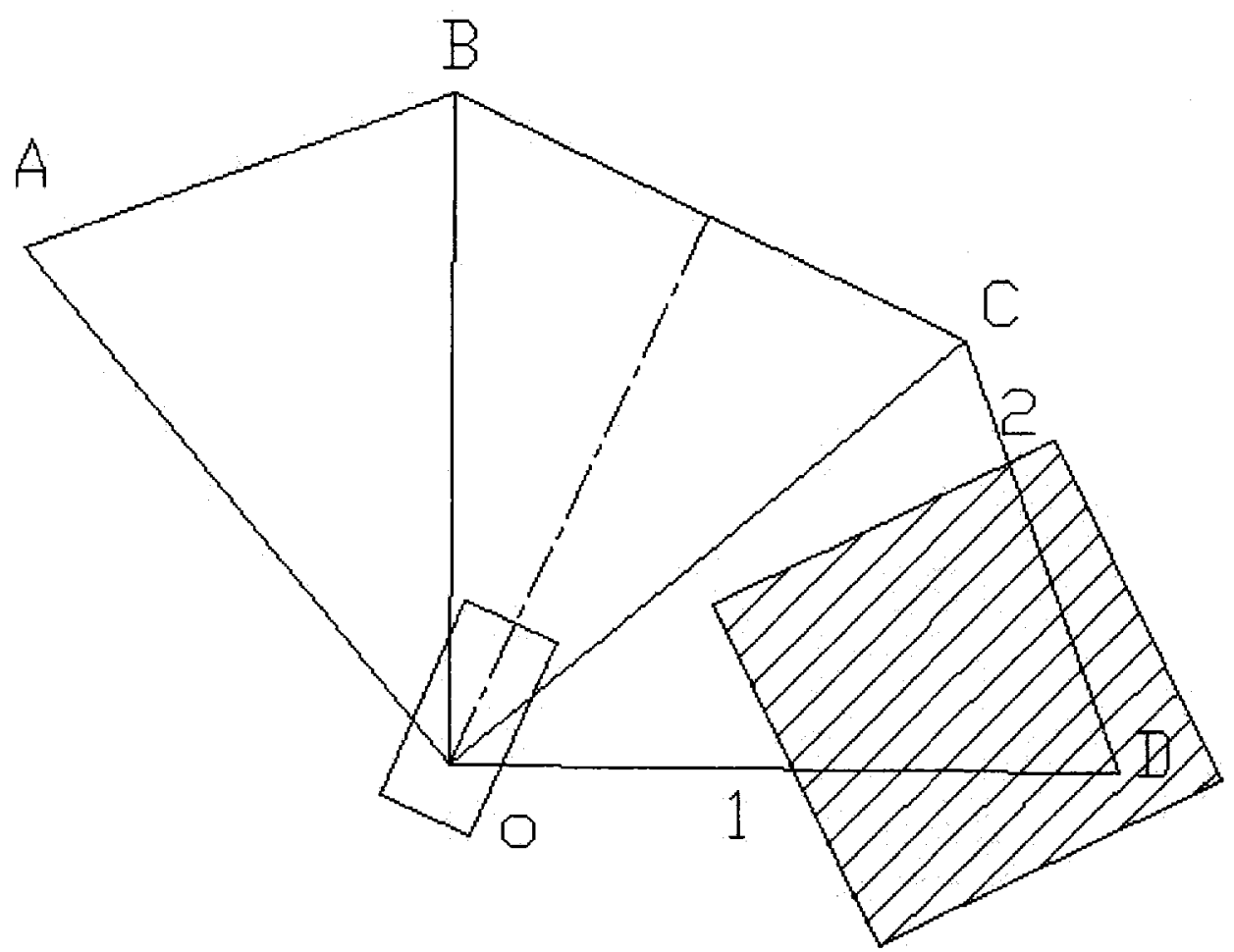

(b)

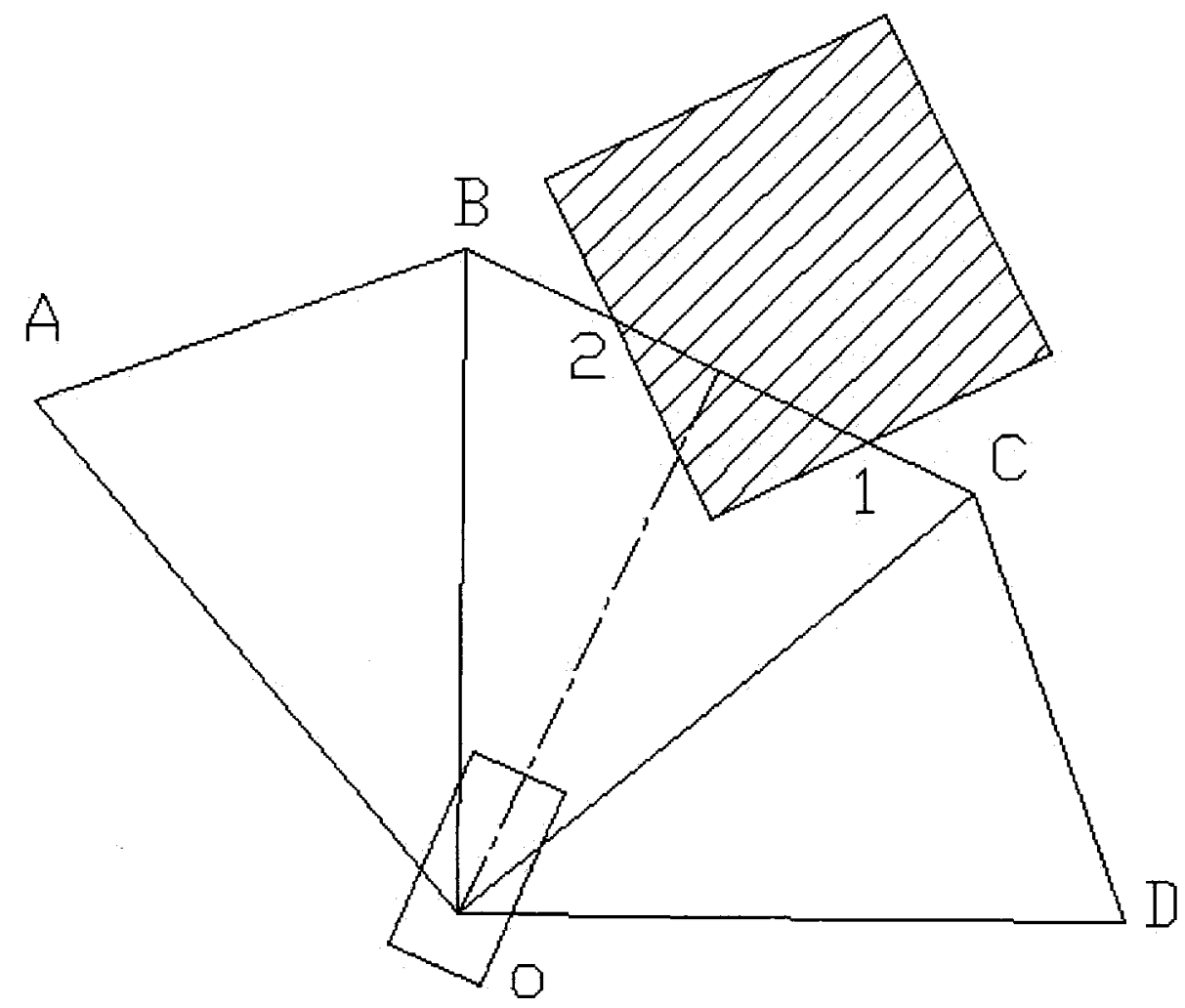

(c)

Figure 6.3: One detection case 
For first and second cases where the obstacle is detected in left and right zone respectively, it can be seen that there is no obstacle in centre and right zones with obstacle on left and no obstacle in front and left zones with obstacle on right. But also by taking into consideration the location of goal point, if the direction of goal point with respect to robot falls in the region same as that of obstacle, a point with some clearance distance away from the selected obstacle end point is fixed as a target point and if not then it is desirable to keep steering angle to zero degree no steering is needed, that is $\delta_{d}=0$. For instance, if the obstacle is in left zone and the direction of goal points also lie in left zone then a point on the plane which is away by some clearance distance from the selected end point of the obstacle is a target point and if not then $\delta_{d}=0$. This is same for either one object or more that one object and for vehicle moving on or away from trajectory. Now a question is what is selected obstacle end point? The selection of end point will be discussed in next section in which either left or right end point of an obstacle is selected based on certain logic. In third case, as shown in figure $(6.3, c)$, the obstacle is detected in front zone..

\section{Algorithm for single detection, left or right case}

If obstacle is in left (or right) zone

If direction of goal in left (or right) zone

Select required target point from selected end point.

Determine steering angle directing to target point

Else

Steering angle equal to zero

End. 
Regardless to number of objects a condition is checked for whether the robot is on trajectory or away from trajectory. If the robot is on the trajectory then one more condition is checked and that is to check if the obstacle is not within 2 meters either side of longitudinal axis of the robot as shown in figure below, it will continue to move on trajectory without bothering about presence of obstacles and if it's within 2 meters, then a target point is selected based on selected obstacle end point. The basic idea of this condition is to keep robot's motion undisturbed till obstacle is found on its way or near to trajectory. This is based on the simple logic when a driver of a car will have, that why need to turn if the obstacle anywhere around the vehicle is not on its way. Also, this approach helps to reduce computational time at every time step which is not necessary and also increase stable movement by not reacting with detection of obstacle that is far away from its path. If the robot is not on trajectory then, the selected obstacle end point previously determined is replaced by left end point of an obstacle. This left end point is also called as second point and the first point will be right end point of obstacle. 


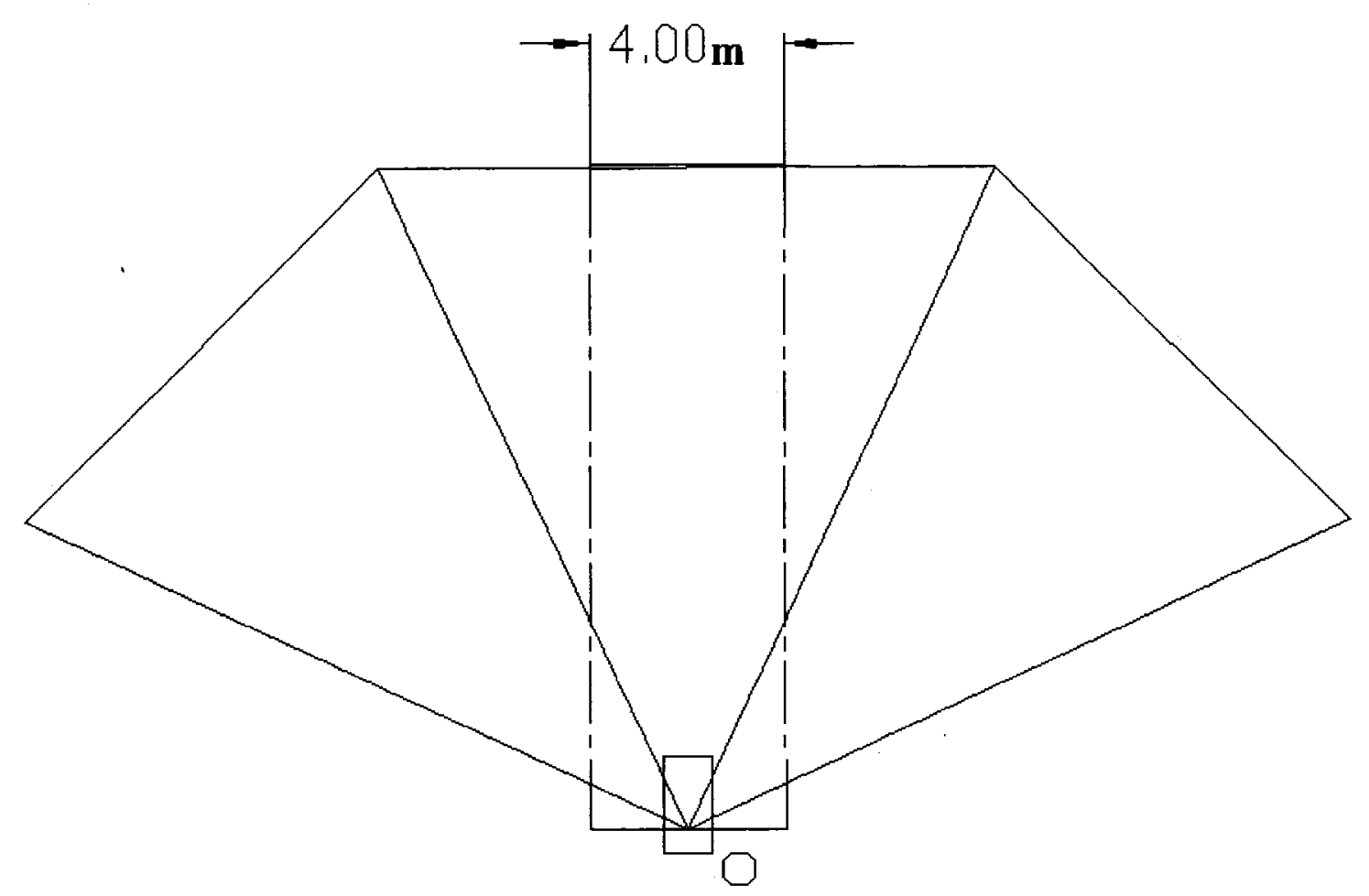

Figure 6.4: Collision area on either side of longitudinal axis

\section{Algorithm for single detection, front case}

If robot is on trajectory

If obstacle is within distance of 2 meters on either side of longitudinal axis Select required target point from previously determined selected obstacle end point*

Else

End

Continue to follow trajectory without bothering about obstacles

Else

End

Selected obstacle point will be 2 .

* = selection of obstacle end point algorithm is explained in next section 


\section{2) Two detection case}
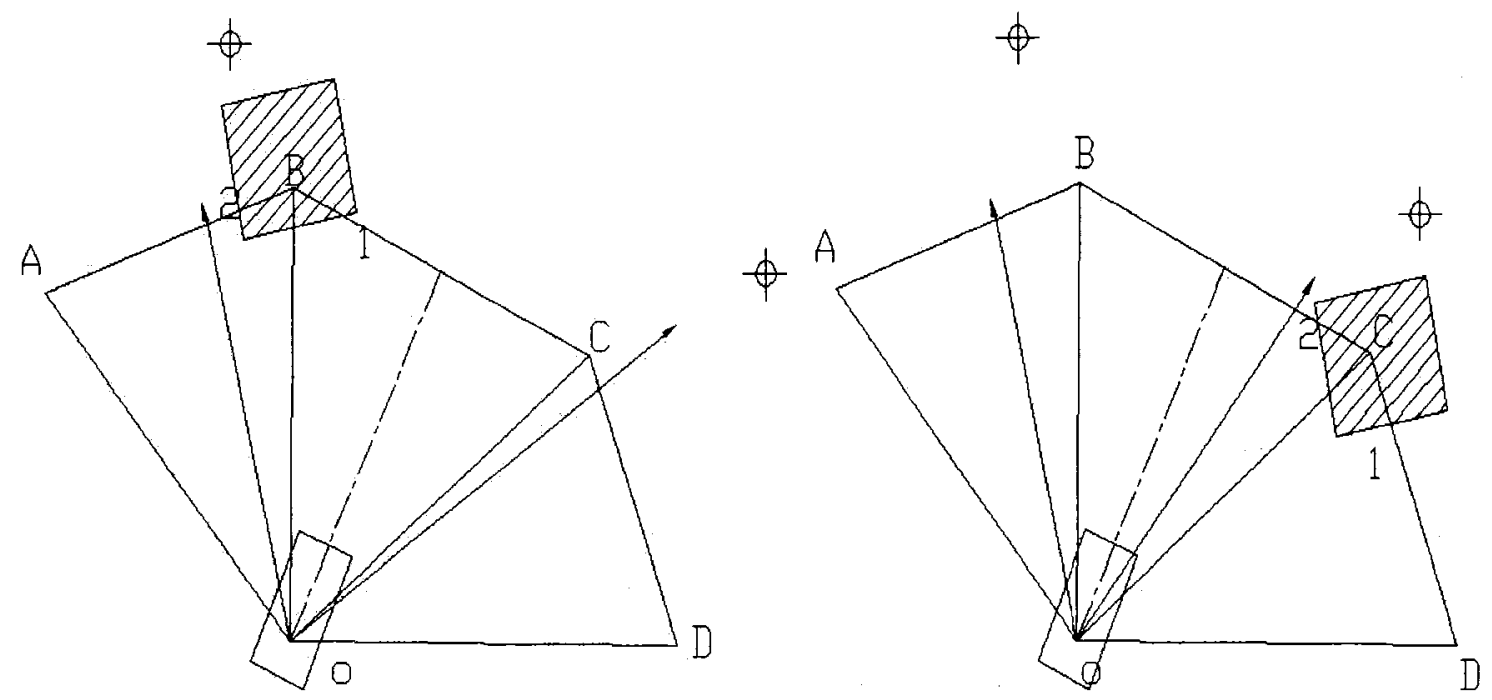

Figure 6.5: Two detection single object

Here, we shall discuss the problem separately for single and multiple objects. First, let's consider with single object case. This problem occur when the object is detected in left and front zones or right and front zones as shown in figure 6.5. Solution for both this problems are similar but opposite in direction. First, as seen in previous case, check if the vehicle is on the trajectory or away from trajectory. If it is on the trajectory then check for presence of object within the distance 2 meters on either side of longitudinal axis or robot. If so then avoidance is achieved by moving away from target point or else move straight without steering vehicle. If it's not on trajectory then check if the direction of goal point lies on same direction as object zone. If yes then target point is fixed based on selected obstacle end point or else move in opposite direction of object. For instance, if the obstacle is detected for left and front zones and the direction of goal is not in that zone than steering angle is -45 degrees (extreme right) and vice-versa. This is similar to 
as we saw with one detection, left/right case, except here instead of moving straight, it moves to extreme right/left.

Algorithm for two detection, single object case:

If objects in right and front zones (or in left and front zones)

If robot on trajectory

If obstacle is within distance of 2 meters on either side of longitudinal axis Select required target point from previously determined obstacle

Else end point and determine steering angle.

Continue to follow trajectory without bothering about obstacles

Else

If the direction of goal is in right and front zones (or in left and front zones)

Select required target point from previously determined obstacle end point and determine steering angle.

Else

Steering angle is 45 degrees (or -45 degrees)

\section{End}

End

\section{End}

With multiple objects, two detections are possible with obstacle present in left and front zones or right and front zones or left and right zones as shown in figure below: 
(a)

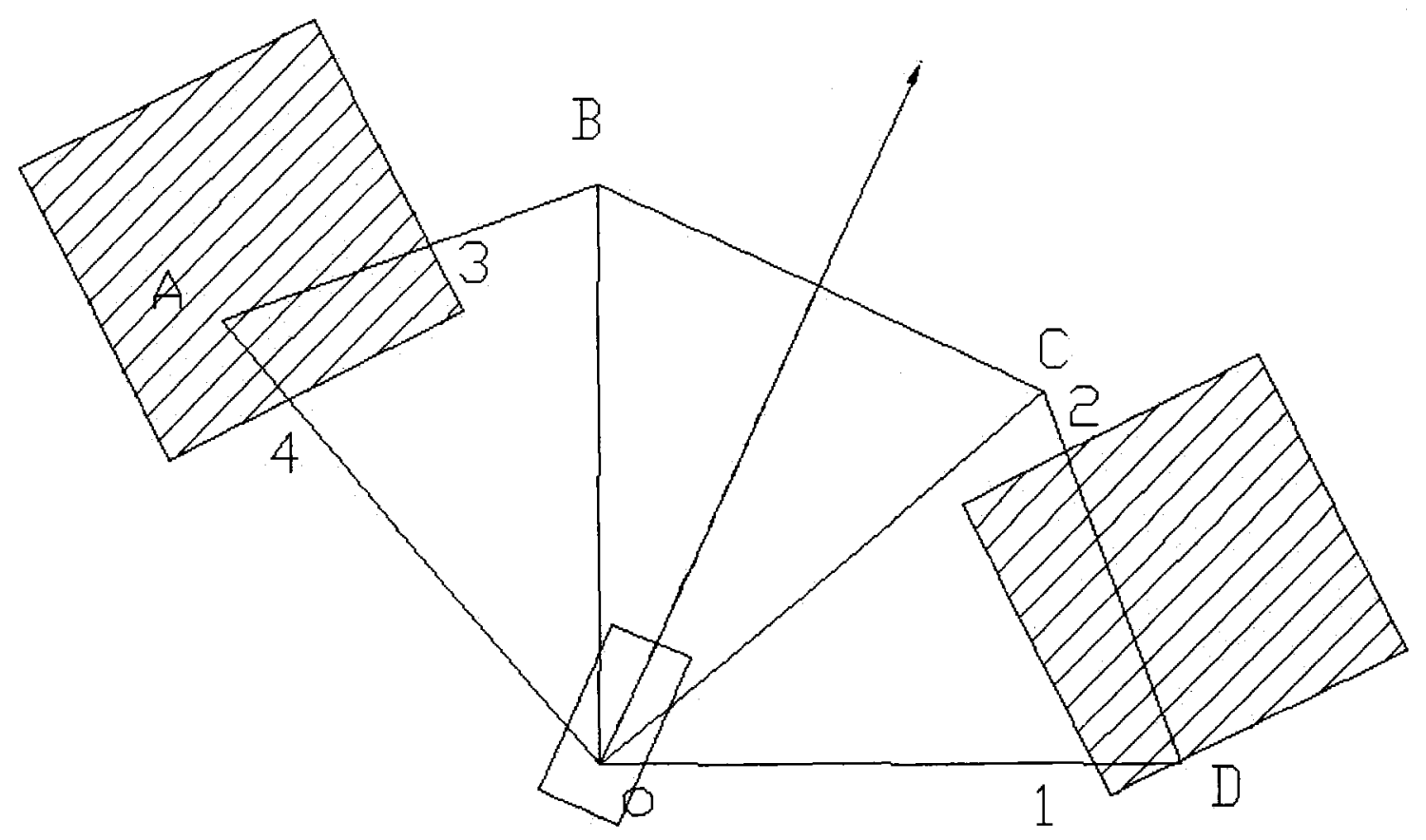

(b)

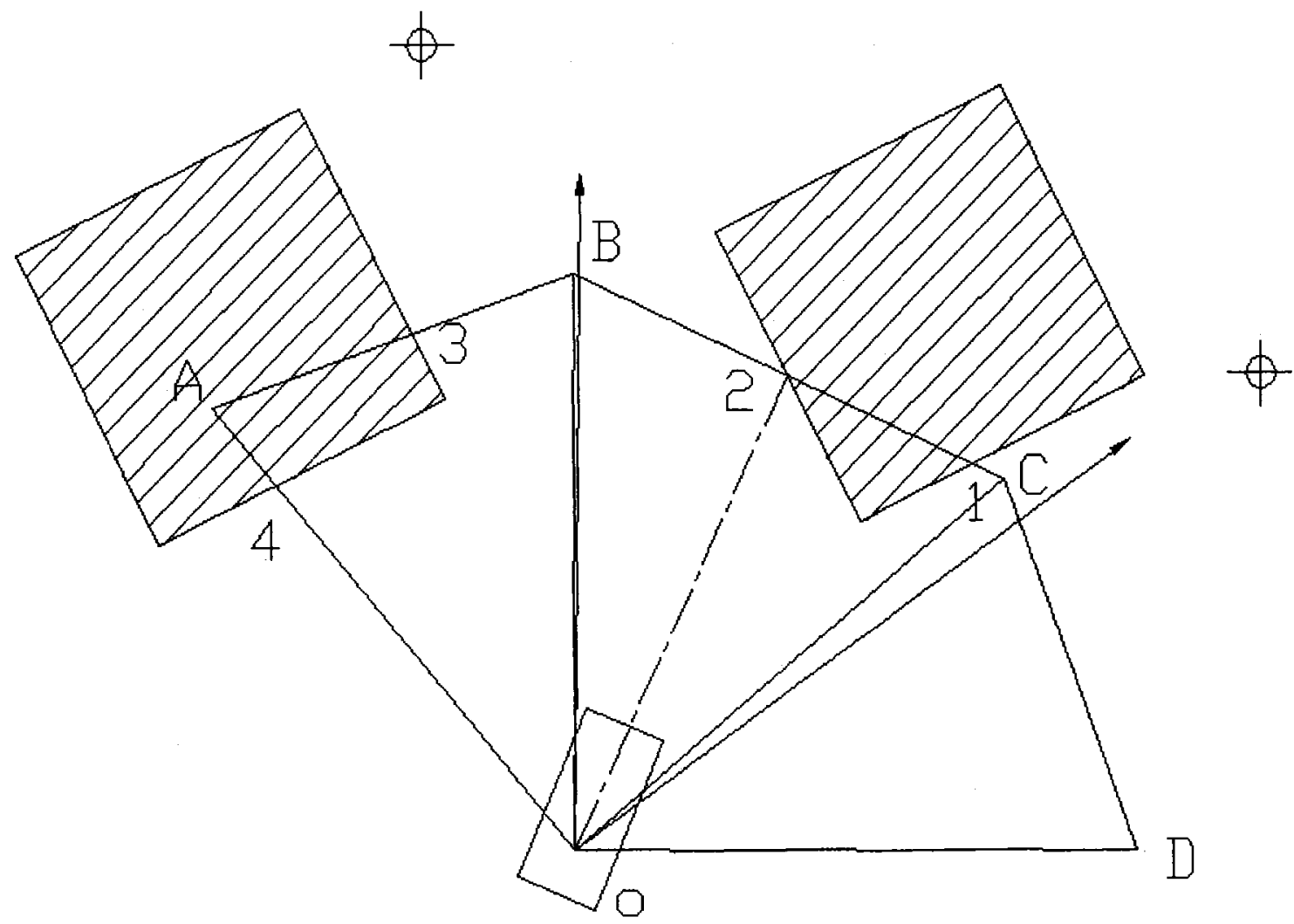


(c)

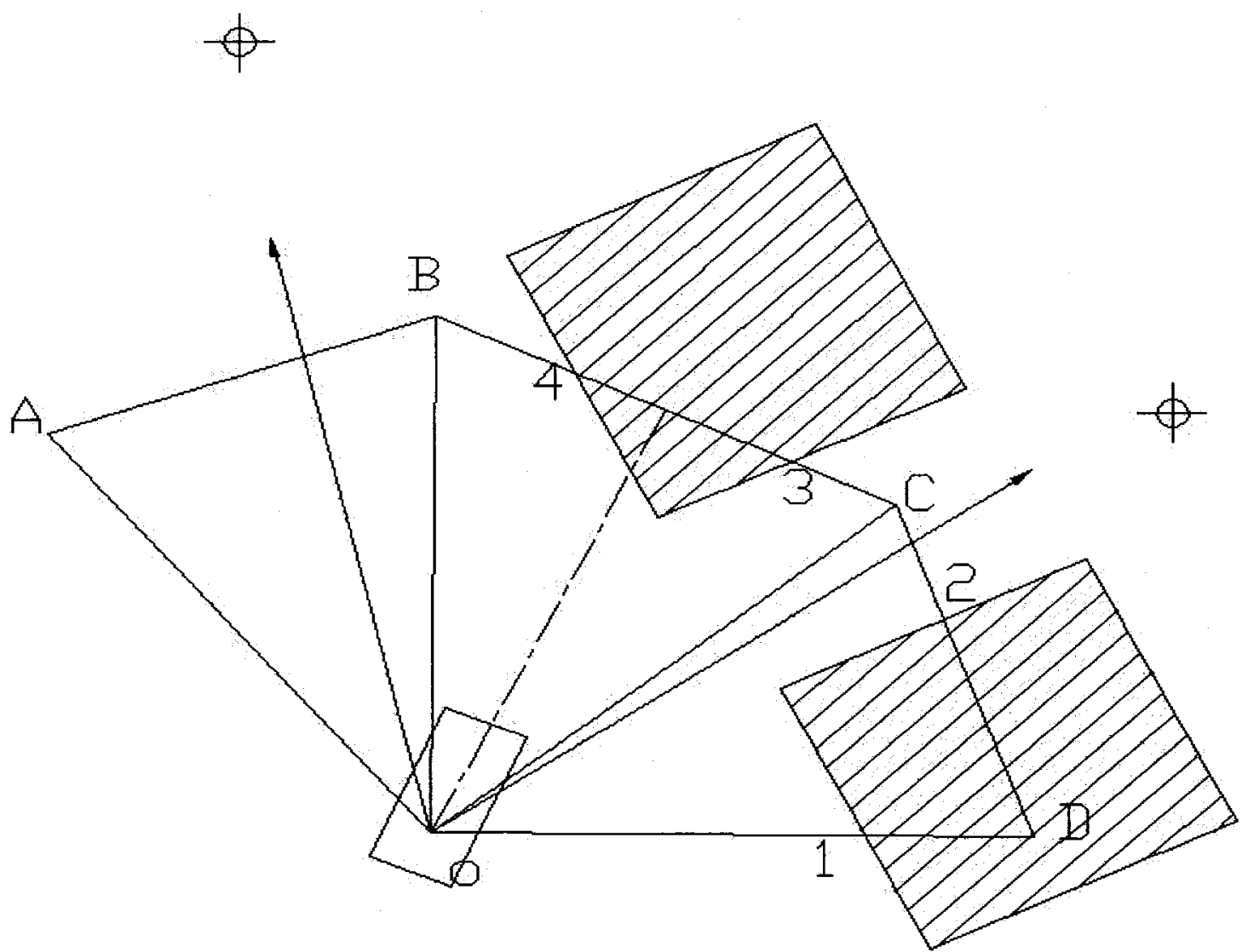

Figure 6.6: Two detection multiple objects.

For condition shown in figure $(6.6, a)$, objects are found on left as well as on right but no obstacle is present in front, the robot is directed to move straight without turning irrespective of robot is on or off the trajectory. For cases shown in figure $(6.6, b)$, and figure $(6.6, c)$, if the robot is on trajectory, the same algorithm is followed as described for previous two cases, that is, if object present within 2 meters on either side of robots axis. If for the robot is not on trajectory and considering case as shown in figure $(6.6, b)$, if the selected point is first point (right end point) of first object then the target point will be some safe distance away from that point, similarly if the selected point is second point (right end point) of last object then a target point in its vicinity is selected. If the end 
point of any objects selected other than these two then the direction of steering angle will be the one which bisects the angle made by end points of two neighboring objects. For instance, if the selected obstacle end point is second point of first object then the steering direction will be one that bisects the angle made by second point of first object and first point of second object with the reference point of robot. In this way the desired steering angle is such that, the robot can pass through the centre of two objects. If the direction of goal is not in obstacle zone then steering angle will be at extreme end opposite to obstacles. Similar is the case with figure $(6.6, \mathrm{c})$ but in opposite direction. The numbering of points and objects are in anticlockwise direction.

\section{Algorithm for two detection, multiple objects case:}

If objects in right and left zone

Else

Steering angle equal to zero

If robot on trajectory

If obstacle is within distance of 2 meters on either side of longitudinal axis Select required target point from previously determined selected obstacle end point.

Else

End

Continue to follow trajectory without bothering about obstacles

Else

If selected end point of object is first of first object Select required target point from previously determined selected obstacle end point and determine steering angle

Else if selected end point of object is second of last object Select required target point from previously determined selected Else obstacle end point and determine steering angle

Select steering angle that bisects the angle made by two end points of adjacent objects and robot reference point.

\section{End}

\section{End}

\section{End}




\section{3) Three detection case}

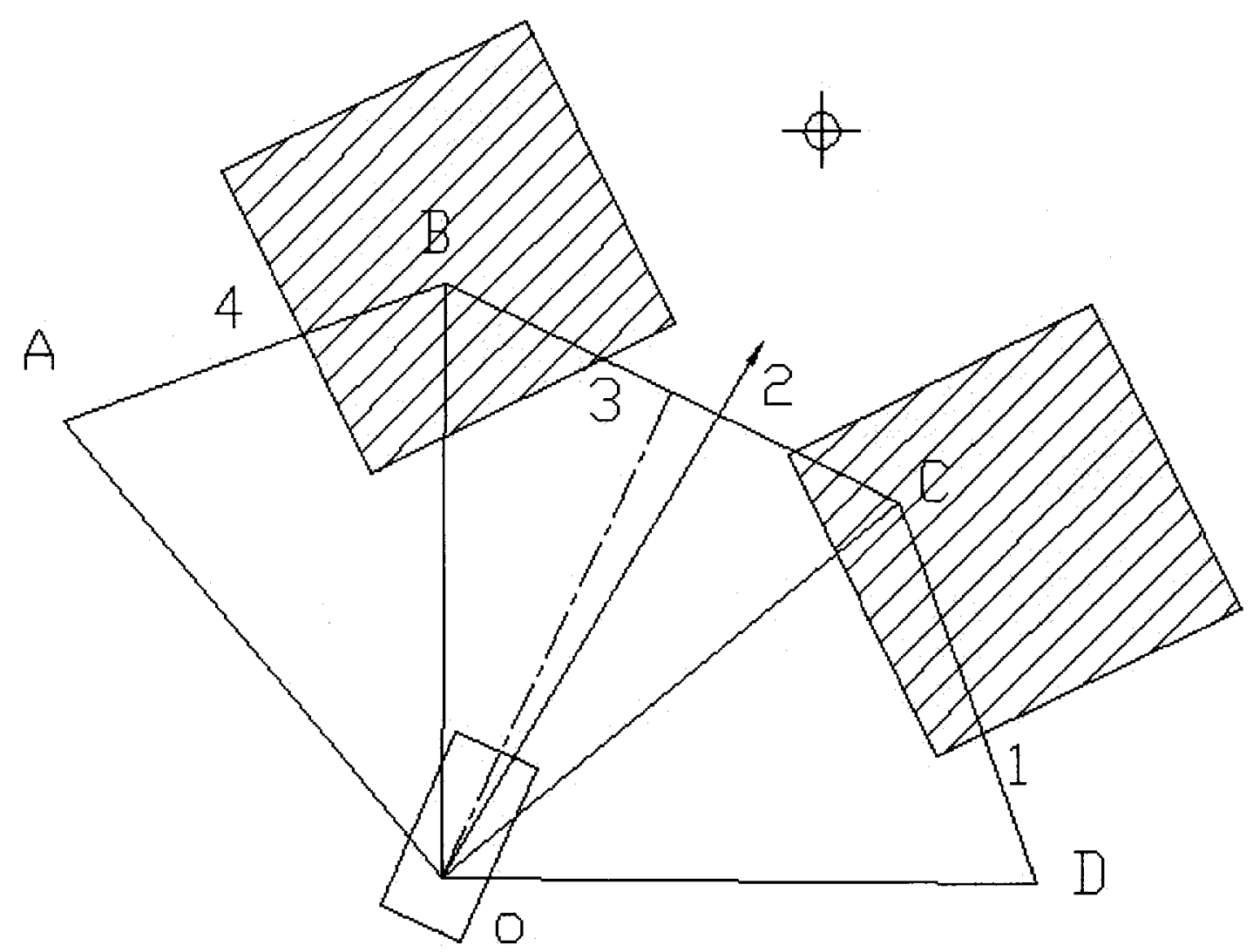

Figure 6.7: Three detection case

This case is same for robot on or off the trajectory. For single object if the goal point is found behind the object the target point will be in vicinity of second (right) end point of obstacle at some safe distance and accordingly steering angles are calculated. Calculation of steering angle is discussed later in this chapter. If the direction of goal point is not behind the obstacle then steering angle is decided based on selected obstacle end point and hence the target point. In case of multiple objects the algorithm is same as that we have seen in two detection, multiple objects case. 


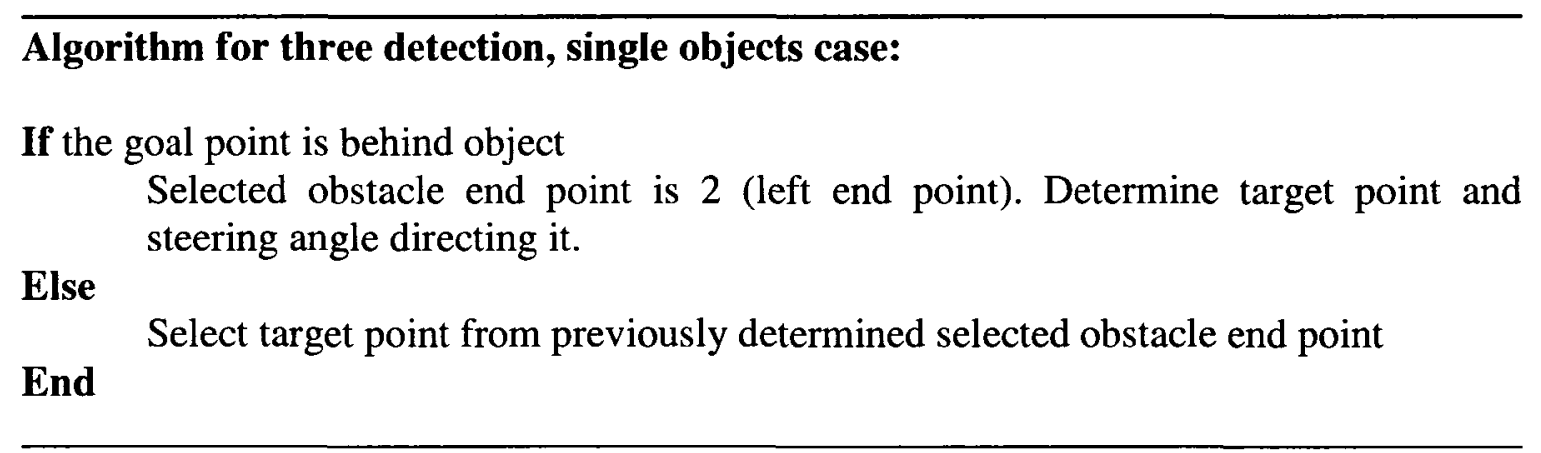

\subsubsection{Selection of obstacle end point}

In the previous section we mentioned about fixing a target point at safe distance from the selected obstacle end point. Here we will see how this obstacle end point is selected and how target point is fixed at a safe distance away from the selected obstacle end point.

Selection of obstacle end point depends on following factors:

1) Robot is moving on or away from the trajectory

2) Location or presence of goal point behind the obstacle.

Selection of obstacle end point depending on first factor can be explained by considering the figure as shown below: 
(a)
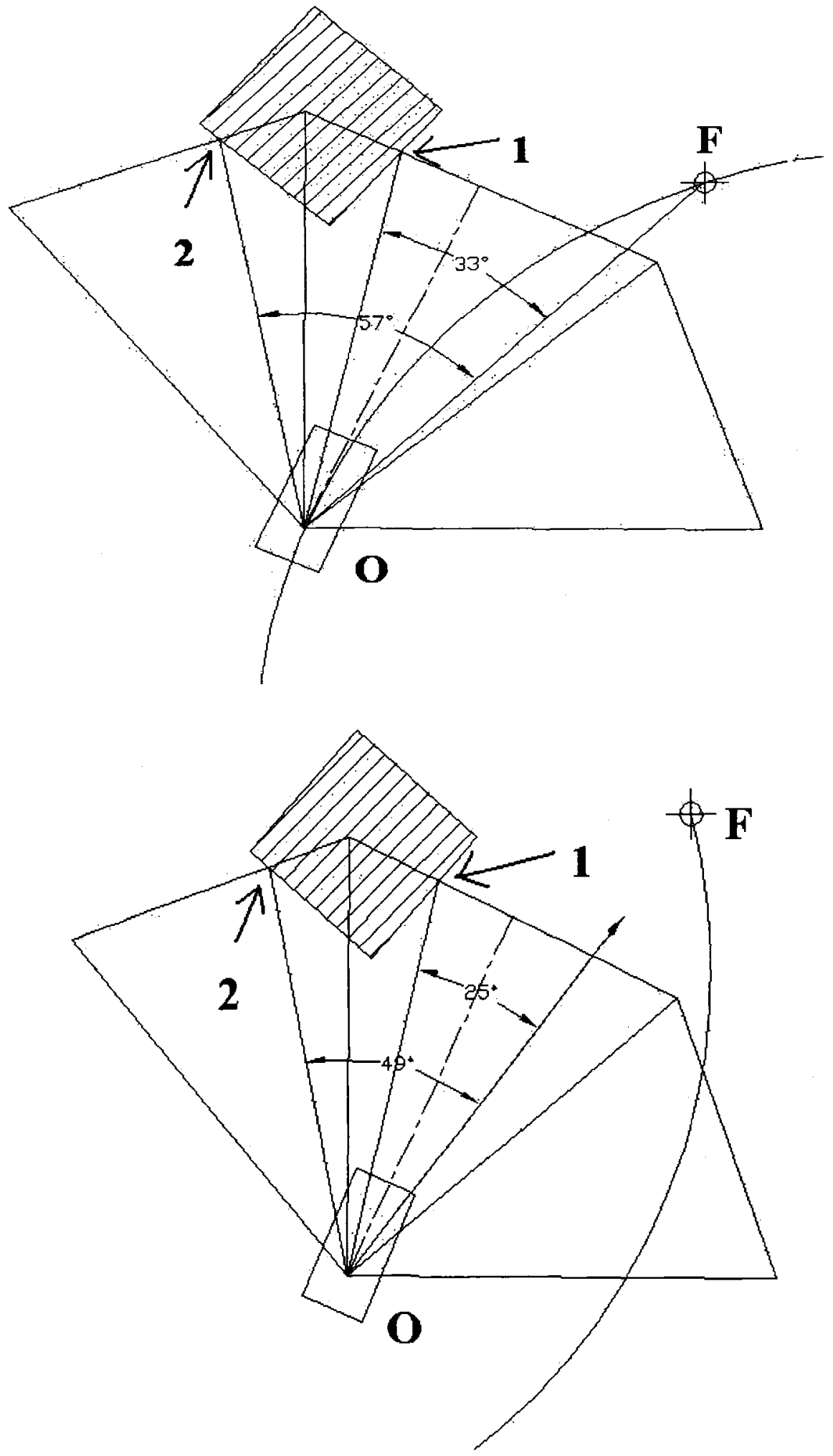

(b)

Figure 6.8: Selection of obstacle end point 
If the robot is on the trajectory and collision is detected, a program will search the point on the trajectory with is $18-22$ meters away from the robot and also nearer to the final goal point. Since we know the local angles of the all obstacle points (those which are visible during each scan), local angle of this goal point is determined and the difference between the local angle of end points of obstacle and goal point is calculated. The end point that forms the minimum angle will be selected as an obstacle end point. This is done to ensure that the robot remain as close to the trajectory. For single object as shown in figure above, the required obstacle point will be first point (numbering of point is done in anticlockwise manner) as it makes smaller angle with the goal point on trajectory as compared to second point. In case of more objects, comparison will be made among end points of all obstacles. Here we note that, the first and last points of an obstacle are decided among only those points that are viewed in each scan and not the actual end points of obstacle. When the robot is away from the trajectory, unlike, on the trajectory, final goal point was considered instead of point at a distance of 18-22 meters from robot reference point.

If the goal point lies behind the obstacle only in case of front detection then the selection of obstacle point is fixed to second point (left end point) of an object in all conditions irrespective to number of objects and location of robot either on or away from trajectory. For second factor consider a figure shown below: 


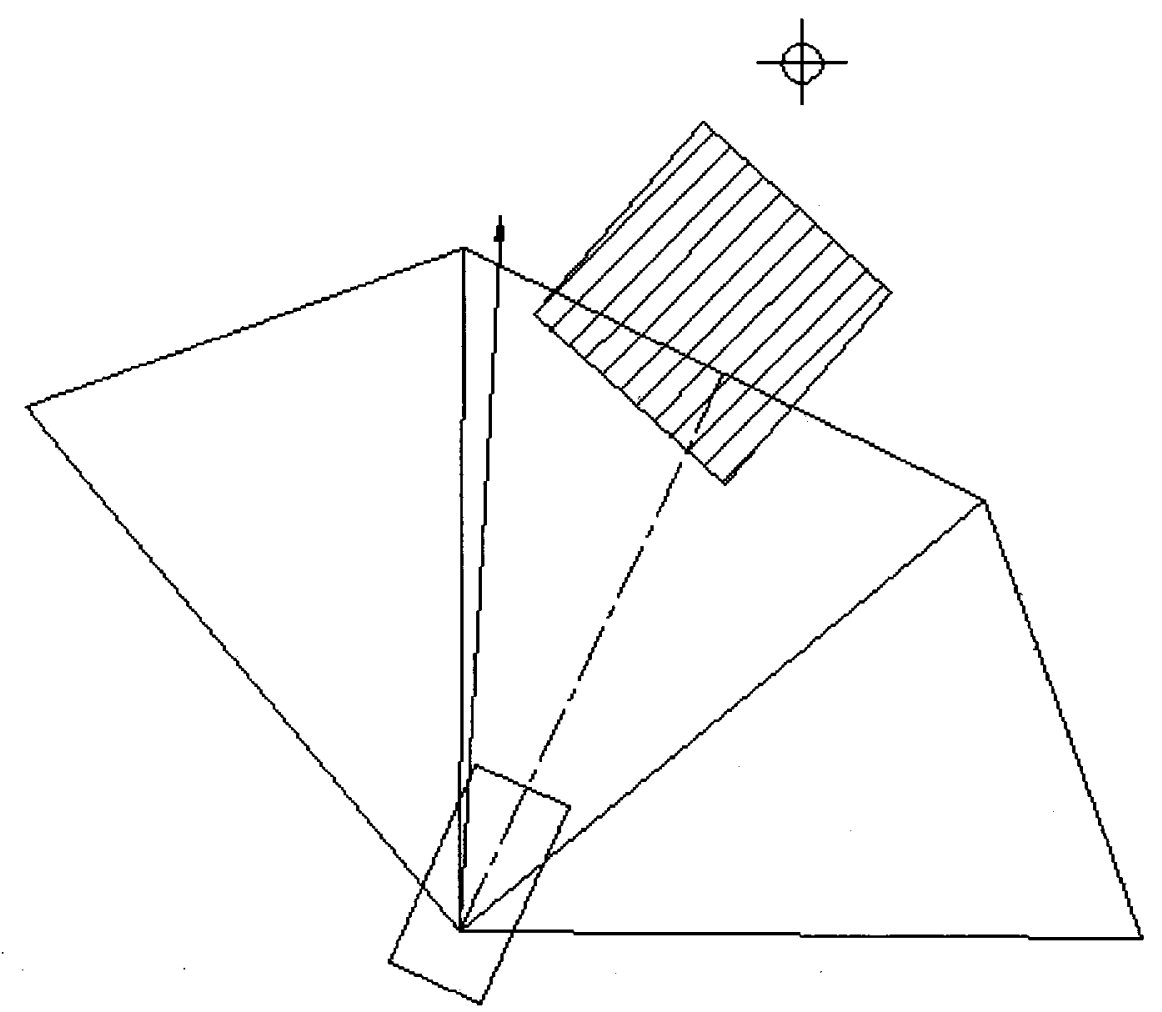

Figure 6.9: Selection of obstacle point with goal behind object

\subsubsection{Selection of target point}

Target point is one on basis of which robot decides the steering angle to maneuver itself during next step. Selection of target point basically depends on situations whether collision is encountered or not.

First we see the selection of target point when collision is detected. If the selected obstacle end point, as discussed in previous section, is fixed as a target point then obviously robot will move towards it and will thrash into the obstacle. To prevent this, some point located at some safe distance from selected obstacle end point is assumed as a target point and steering angle is determined by adding safe angle to steering angle 
determined with respect to selected obstacle end point. Selection of safe distance depends on design and application. More is the safe distance, more will be the clearance between robot and obstacle, but tends to move away from the desired trajectory. Hence safe distance should be selected optimally depending on application. Here in this work 3 meters is chosen as safe distance. The width of robot should be taken into consideration while selecting this distance.

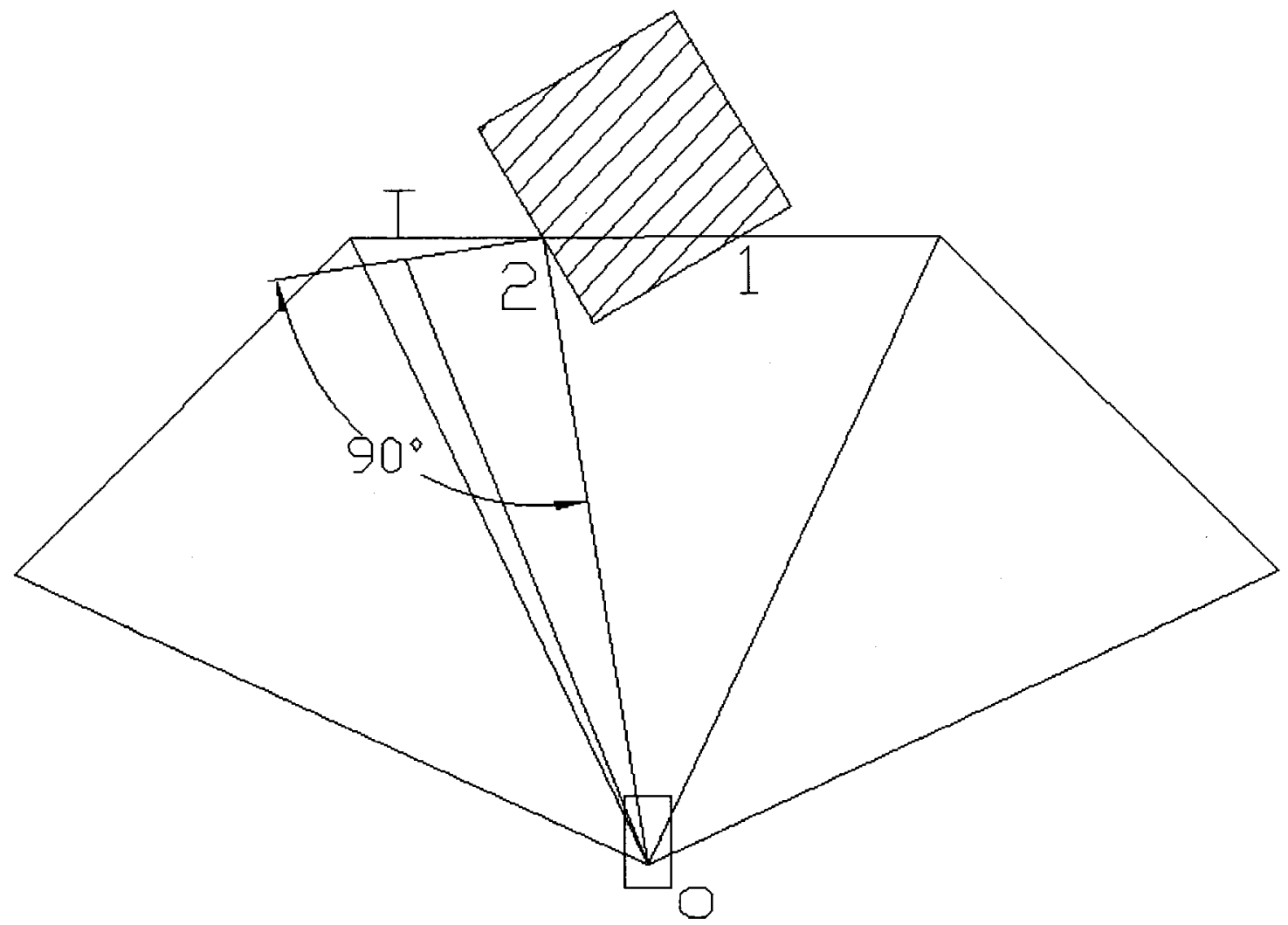

Figure 6.10: Selection of target point when collision is detected

Second situation is when collision not detected and robot either has to come on trajectory or has to follow when it is already on trajectory. If the robot is on the trajectory then the target point will be among equidistant points on the trajectory and the selection of particular point at particular situation vary from one particular trajectory to another 
(straight or curved). The selection criterion is fixed by carrying out trial and error method for particular trajectory and once it's decided, the algorithm for that criterion is provided to robots memory. Since in this work, robot moves with constant velocity, it should be kept in mind while formulation of target point selection criterion that there should be no major overshoots during path tracking. For instance, if the target point selected is very close to current robot pose then the situation may arise where the time required by the robot to reach that point will be less than simulation sampling time. In that case there will be on overshoot or deviation from robots path. On the other hand, if the target point selected is very far then, the path curvature in between robot and target point is neglected and this cause robot to deviate if the path is not straight. Hence, the point is selected in such a way that it is bit more than the distance the robot can travel with that fixed velocity in time of simulation.

If it's not on trajectory then the point on trajectory which makes shortest distance is selected as target point. Now, the question comes that when and how robot should switch between these two different selection criteria for on and away from the trajectory. The selection of target point during this intermediate or transition stage is critical as again if it is selected when robot is just near to trajectory but not on the trajectory by selection based on minimum distance, then it is possible that it will not converge with the path smoothly and will form on overshoot and will fluctuate about its path till it settle down after some time. Hence the goal point on trajectory should be selected in such a way that some points are added to the point located at minimum distance as it approaches closer to trajectory. This also applies to the case when robot has just passed away from obstacle 
and wants to come back on trajectory. The figure below differentiates among these situations.

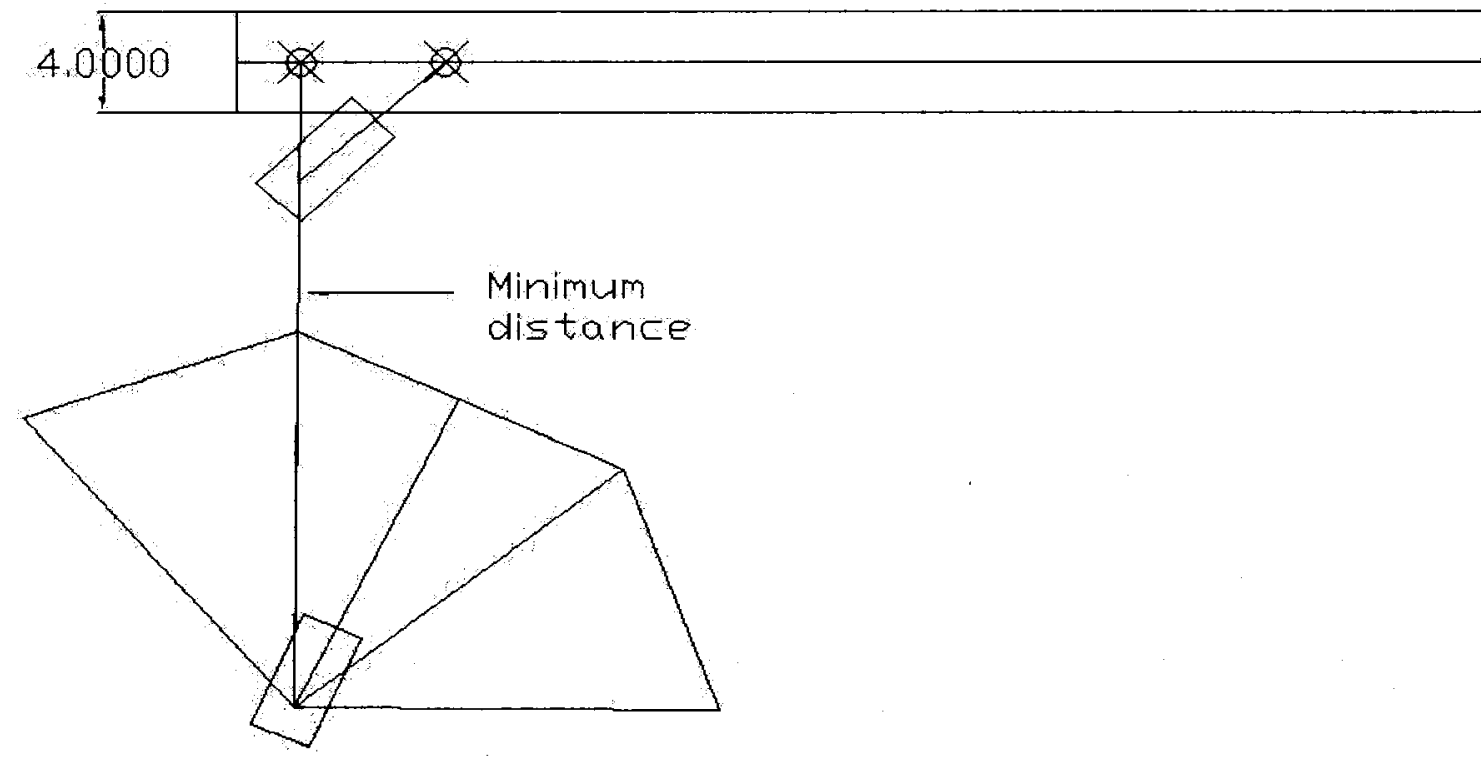

Figure 6.11: Different situations for selection of goal point on trajectory.

\section{Algorithm for selection of target point:}

Step 1: Determine if collision is detected or not.

Step 2:If collision detected

Else

Fix the target point safe distance away from selected obstacle end point.

Determine if the robot is on or away from trajectory.

If robot is on the trajectory

Fix the target point that does not generate overshoot.

Else if robot is far from trajectory

Fix the point on trajectory that makes minimum distance with

Else current robot pose as target point.

Fix the target point (some points added to point making minimum distance) that cause it to merge smoothly with trajectory without overshoot

End

End 


\subsubsection{Determination of steering angle}

If we consider a robot a point moving in the plane and if the orientation with respect to reference axis is not considered then the steering angle can be determined as:

$$
\begin{aligned}
& d x=\left(x-x_{t}\right) \\
& d y=\left(y-y_{t}\right) \\
& \alpha=\tan ^{-1} \frac{d y}{d x}
\end{aligned}
$$

$x_{t}=\mathrm{X}$ coordinates of goal point,

$y_{t}=\mathrm{Y}$ coordinates of goal point

But since, it is not in this case here, steering angle can be computed by considering four different cases: orientation of robot in first, second, third and fourth quadrant respectively. For calculation of steering we first need the information regarding the goal point, that is, its location in $\mathrm{X}-\mathrm{Y}$ plane. In addition to its location, the orientation of this goal point with respect to robots frame is also required which is determined using equation (6.3). Four different cases for determining steering angle are shown in figure below: 


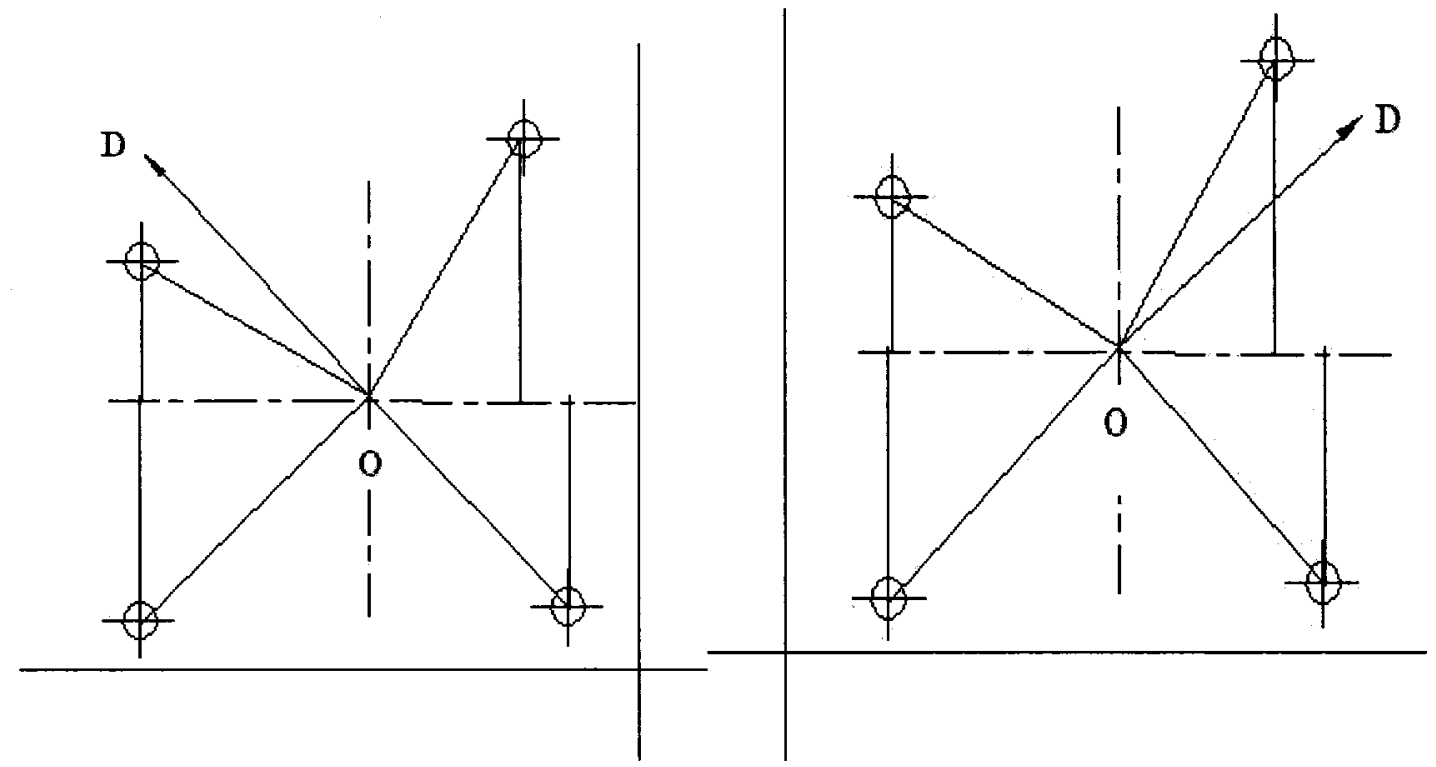

(a) Orientation in second quadrant

(b) Orientation in first quadrant

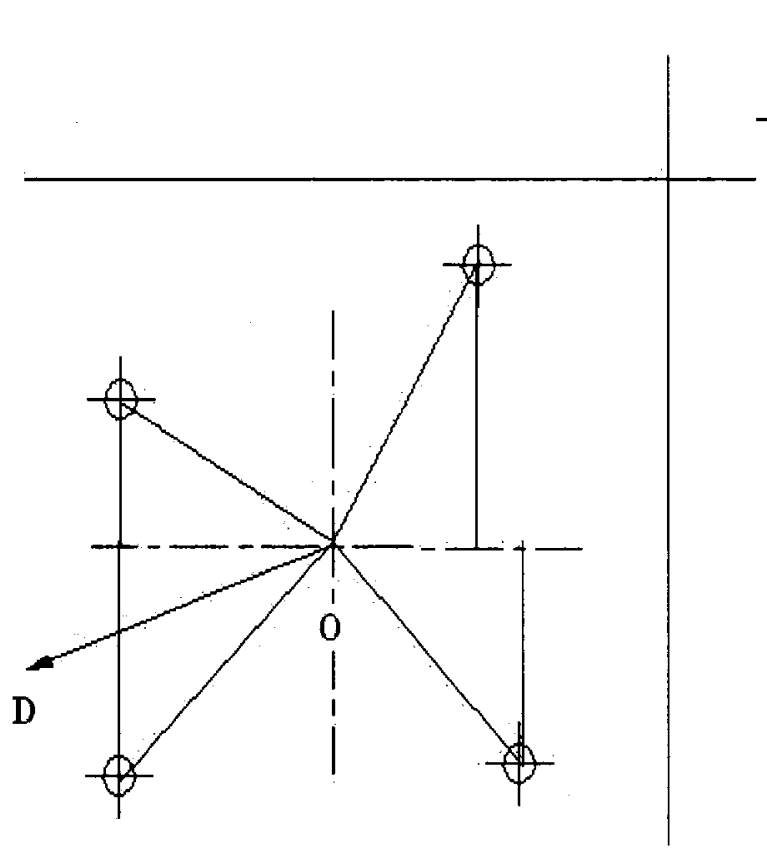

(c) Orientation in third quadrant

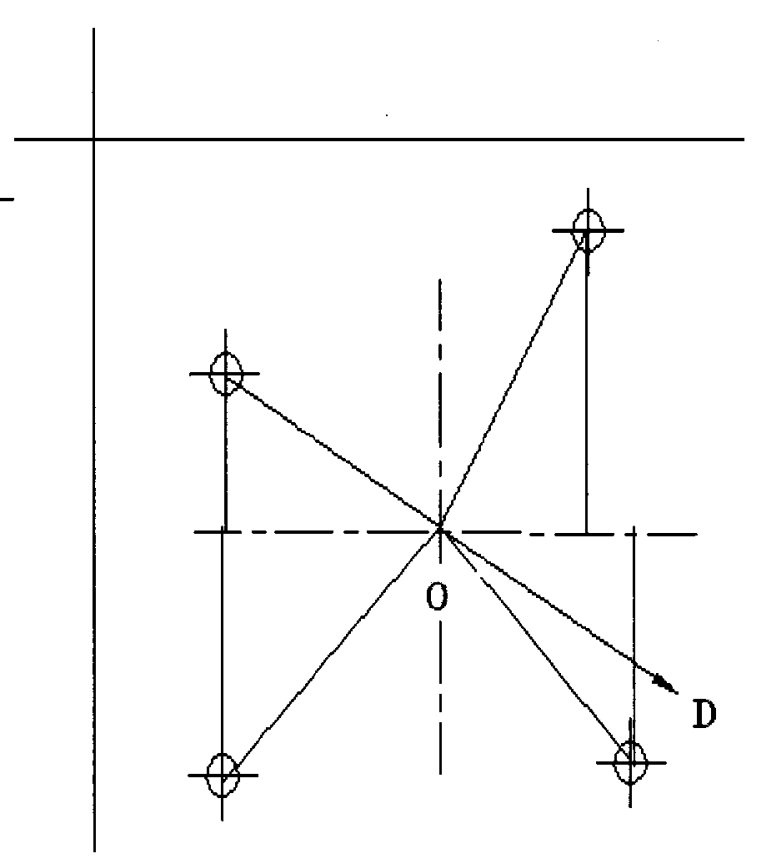

(d) Orientation in fourth quadrant

where, $\overrightarrow{O D}=$ Heading direction of a robot, $O$ refers to robot's reference point $\phi$

$=$ Four probable goal point locations in each case

Figure 6.12: Robots orientation in different quadrants. 
1) When orientation is in first quadrant. $(0.00001 \leq \theta \leq 90)$

If $d x \geq 0$

Desired steering angle $=\alpha-\theta$;

Else

Desired steering angle $=180+\alpha-\theta$;

End

2) When orientation is in second quadrant $(90<\theta \leq 180)$

$\theta=180-\theta$

If $d x \geq 0$

Desired steering angle $=\theta+\alpha-180 ;$

Else

Desired steering angle $=\theta+\alpha$;

End

3) When orientation is in third quadrant $(180<\theta \leq 270)$

$\theta=\theta-180$

If $d x \geq 0$

Desired steering angle $=\alpha-\theta-180$;

Else

Desired steering angle $=\alpha-\theta$;

End

4) When orientation is in fourth quadrant $(270<\theta \leq 359.9999)$

$\theta=360-\theta$

If $d x \geq 0$

Desired steering angle $=\alpha+\theta ;$

Else

Desired steering angle $=\alpha+\theta-180$;

End 


\subsubsection{Presence of close obstacles}

During collision avoidance robot might encounter a case when the object comes very near to the robot due to nonholonomic constraints or unexpectedly placed object in front of robot. In that case it becomes necessary to avoid those obstacles that are closer than those who are far off. The distance from the robot within which the obstacle detected is considered as close obstacle depends on the application and is prefixed. On this work, 3 meters from the robots reference point is considered within which if obstacle found, priority has to be given to avoid those obstacles that those present beyond 3 meters. This can also be viewed as a protection shield in front of robot and the distance is the radius of that shield. When any obstacle is detected within this region, the steering angle is determined to avoid this obstacle such that the new steering angle determined makes 90 degrees with the line connecting the obstacle point with robots reference point in direction opposite to that of obstacle point. The width of robot here is 1 meter hence this close distance selected will be able to protect robot by 1.5 meters from front and 2.5 meters from side ways. The advantage of selection of this safe distance is that it will avoid collision in most of the cases but on the other had it suffers from the drawback that it might create more fluctuations in steering angle while passing through narrow escapes like the door of width less than 3 meters in our case. The another advantage for adopting this method is avoidance of robot cutting edges of an obstacle while coming back on to trajectory after avoiding collision. The simulation results shown in chapter 7 supports this advantages. 


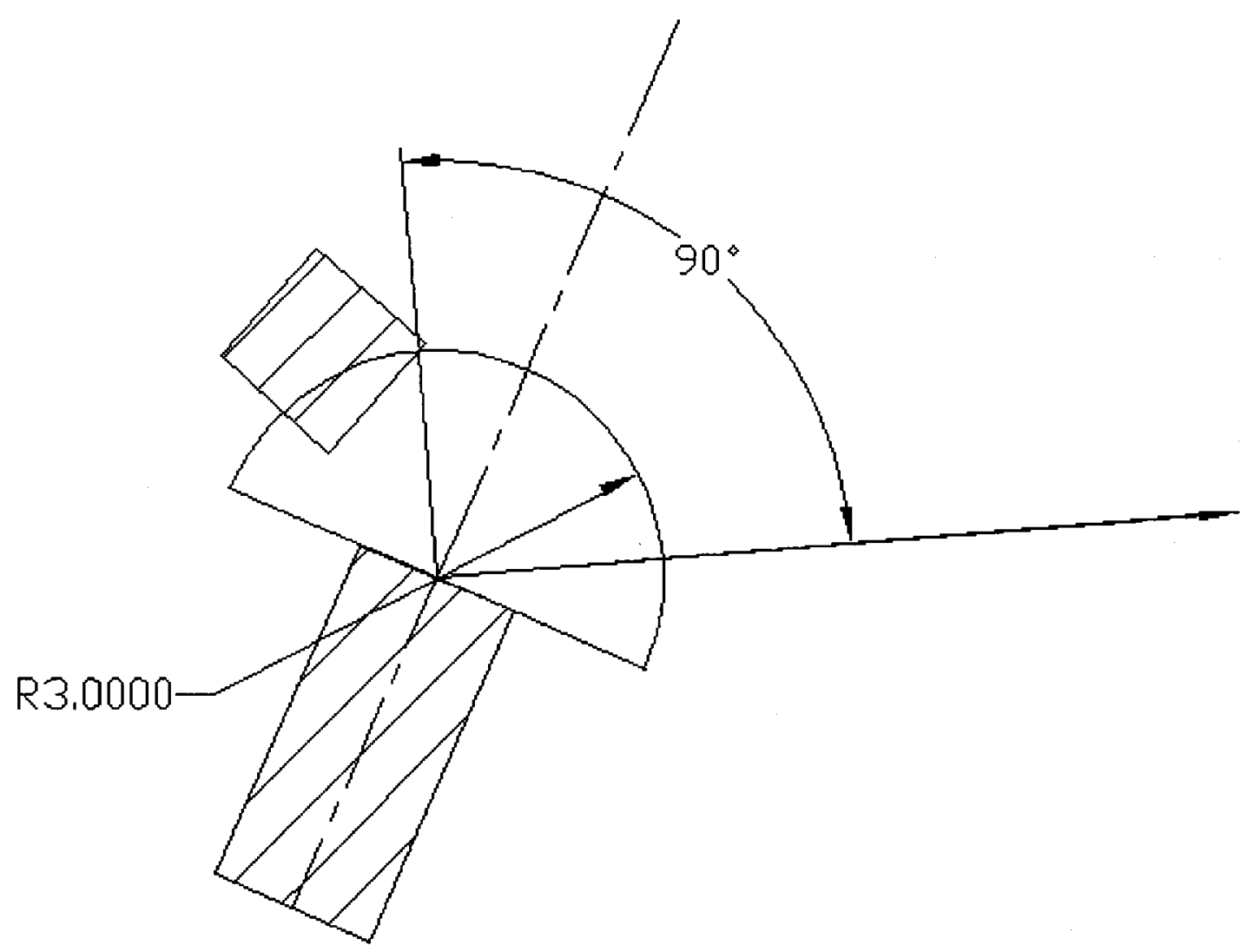

Figure 6.13: Collision avoidance of close obstacles

\section{Algorithm for avoidance of close obstacles:}

Step 1: Determine the presence of obstacle within prefixed close distance.

Step 2: If obstacle is present

Calculate steering angle making an angle 90 degrees with a line obstacle point and reference point in opposite direction of obstacle point.

End 


\subsubsection{Steering controller}

As mentioned earlier, steering controller used during collision avoidance is different from that we used for path tracking. Earlier in case of path-tracking we designed controller the gains of which are generated using LQR but in this case algorithm is switched and only steering offsets are used by simple PD controller. The proportional gain is fixed to 10 . After avoiding collision, the robot needs to come back to original trajectory. As discussed earlier in section 6.2.3 we discussed the selection of target point, during collision avoidance as well as during path-tracking and the steering angle required reaching that target point is determined. When the robot is on the trajectory with no collision, we provide steering, orientation and distance offset but during the situation when robot is not on trajectory and coming back on it after avoiding collision, steering angle and distance offsets are given in same manner but orientation offset is set zero till object reaches within range of 5 meters from trajectory. This is done in this case to ensure the robot reaches the minimum distance point selected and hence to the trajectory as soon as possible. 


\section{Chapter 7: $\quad$ Simulations and results}

This chapter describes in detail the MATLAB simulation environment used for navigation of car-like robot using occupancy grid method. In the simulation, a reference trajectory is generated for the robot to move using differential flatness method. Localization of the robot at every step is discussed in chapter 3. Mapping for acquiring knowledge of the surrounding is carried out by generating occupancy grid with the help of laser range finder is discussed in chapter 4 . Following the given reference path is described in chapter 5 and finally collision avoidance algorithm that determines steering angle for various condition is explained in chapter 6.

\subsection{Simulation background}

The simulation for navigation of car-like robot is carried out with series of programs performing different tasks where data from previous step is utilized by preceding steps. The organization and flow of data is shown in the flowchart as given by figure 7.1. Simulation is started by initialization consisting of creation of reference trajectory, various variables used in simulation, occupancy grids and the objects or obstacles placed in the workspace where robot is operating. This is followed by motion of the robot to the next position using initialized velocity and steering velocity and the new position is estimated using EKF in the next step of program. Laser range finder scans the 2D environment; the data thus obtained is refrained from noise again by using extended Kalman filter. This data is used to gain the information regarding environment 
surrounding the robot by generating occupancy grids. The occupancy grid probabilities indicate the location of obstacles. If obstacles are detected, then local path planner determines the next steering angle so as to successfully avoid the obstacles. For this, steering controller calculates the necessary rate at which the robot should steer. If obstacle is not detected then using the offsets in location and orientation, steering controller and finally velocity controller calculates required values for the robot to move on the predefined path with desired velocity. Finally, when final goal point is reached, simulation is terminated or else these steps are repeated until robot reaches its destination. Following sections will deal with each steps discussed. 


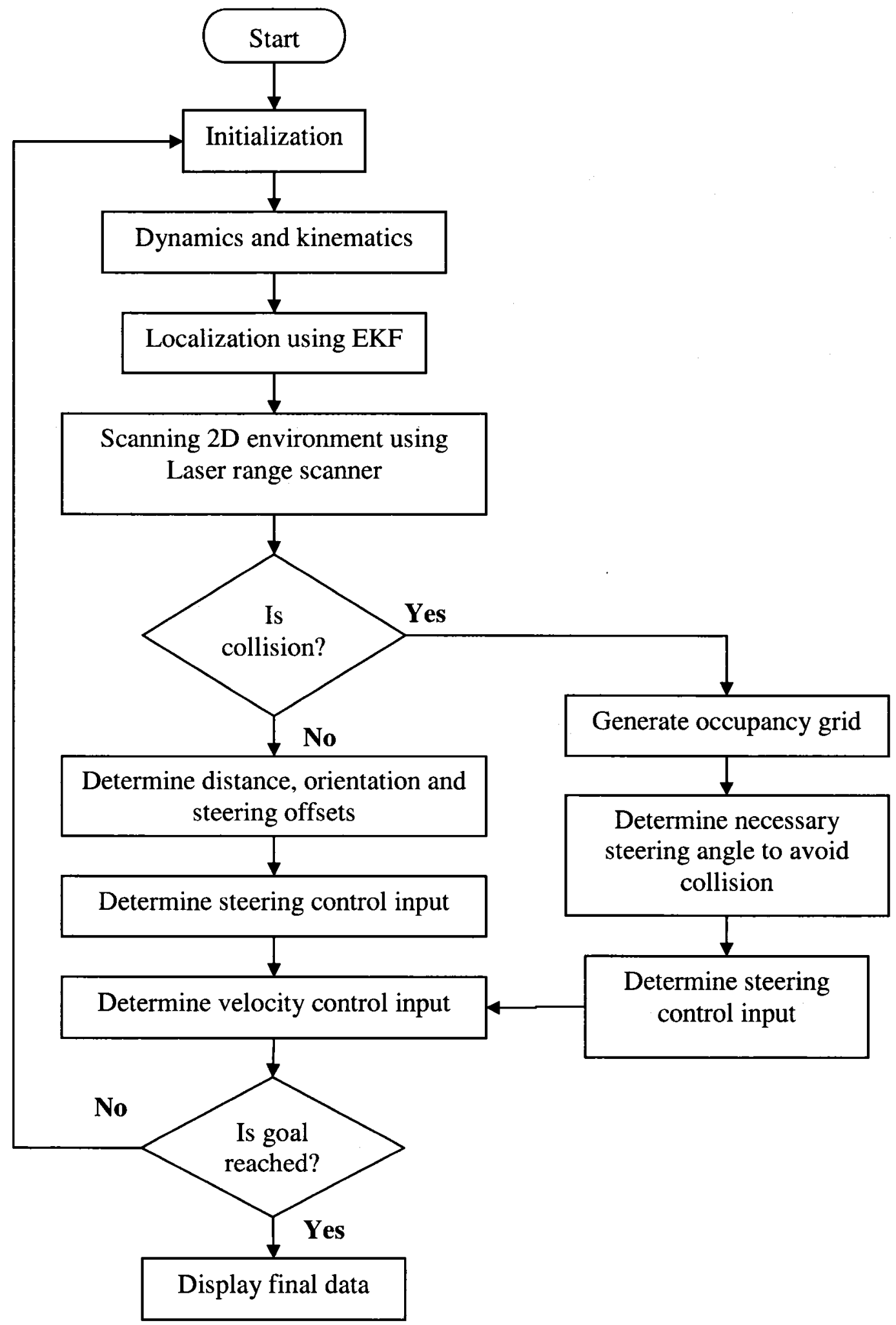

Figure 7.1: Flowchart for MATLAB simulation 


\subsection{Simulation}

\subsubsection{Initialization}

Initialization is a process that has to be carried out at the beginning of every program before it is executed. First step of initialization is to initialize all those variables that carry some value generated by some program and are used by another program in following steps need to be defined. The variables such as offsets (whose initial values are fixed to zero), flags, initial steering angle, and initial error covariance matrix for extended Kalman filter which is kept to product of identity matrix of order three and some constant say 50. The dimension of workspace, vehicle and initial location of vehicle are also fed. Second step is generation of reference trajectory by providing initial global configuration $(x, y, \theta, \delta)$, and final configuration $(x, y, \theta, \delta)_{F}$. In addition to this initial and final time is also provided and reference trajectory is generated using differential flatness method. Simulation is setup to keep only one reference trajectory at all the time. Third step is to initialize the grids with initial probability of 0.5 to every grid cells. The work space is divided into grids with the resolution of 1 meter square. These probabilities are updates at each step to determine presence and location of obstacle. Finally information regarding the objects end points is provided. Simulation is carried out with the polygonal

objects having 4 sides. Using these end points the matrix consisting of information regarding edges parameters, ends, is calculated which is further used by simulated Laser range finder to detect presence of obstacles. Polygonal objects used here are convex. 


\subsubsection{Dynamics and kinematics models}

The dynamic and kinematic equations for car-like robot are given by equations from (5.10) to (5.20). The mass of robot is $100 \mathrm{kgs}$ and moment of inertia is $25 \mathrm{~kg}-\mathrm{m}^{2}$. Both kinematic can dynamic parts forms a full vehicle model. The model uses the current states as the initial condition and integrates it to determine the vehicle's new position after inputs are changed for the next step. It is assumed that the inputs are constant over the sampling time.

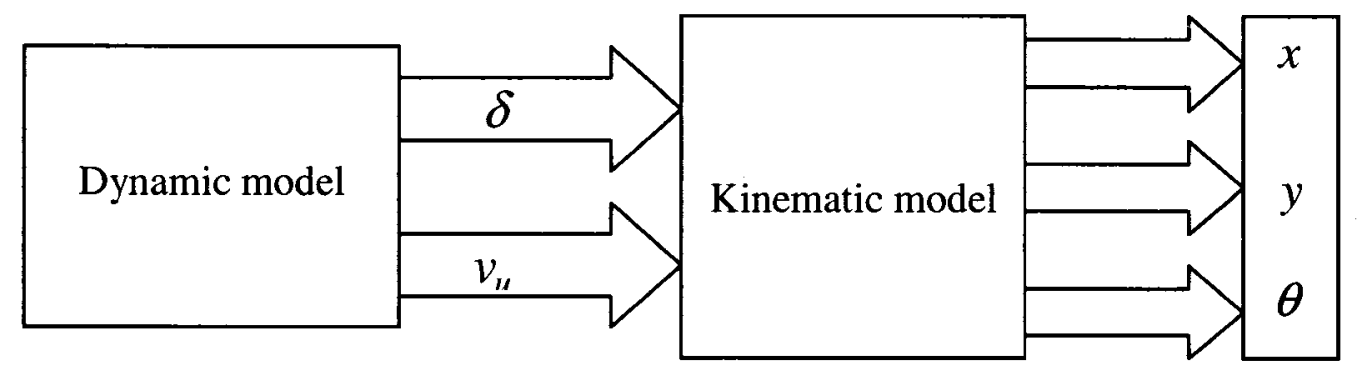

\section{Figure 7.2: Dynamics and kinematics}

Consider the initial position of robot as $\left[\begin{array}{l}x_{0} \\ y_{0} \\ \theta_{0} \\ \delta_{0}\end{array}\right]=\left[\begin{array}{l}8 \\ 22 \\ 45 \\ 0\end{array}\right]$

The parameters of car-like robot are $l=1.5 \mathrm{~m}, a=1 \mathrm{~m}, b=1 \mathrm{~m}$. Let initial velocity is $1 \mathrm{~m} / \mathrm{s}$ and rate of steering is $-1 \mathrm{rad} / \mathrm{s}$. The maximum steering angle is limited to 45 degrees. These parameters are put into dynamic and kinematic model equations (5.10) to (5.20) to get the velocity and steering angle as: $v_{u}=0.1, \delta=-0.1, G_{p}=0.01$ and $G_{s}=0.0097$. The values of $v_{u}$ and $\delta$ is given to kinematic model to give new states as $x=8.0012, y=$ 
22.0012, $\theta=44.9999$. These values are predicted values consisting of system noise and are updated using readings from encoders using extended Kalman filter as shown in next section.

\subsubsection{Localization using extended Kalman filter}

For localization we need to consider that noise is present in the system and in observation from encoders due to various reasons as discussed in chapter 5. Various combinations of $\mathrm{Q}$ and $\mathrm{R}$ matrices as shown in table 5.1 are considered and finally value of $\mathrm{Q}$ matrix was considered to $10 I_{3}$ matrix and $R=500 I_{3}$ and the simulation for these $Q$ and $R$ is shown in chapter 5. For different values of $\mathrm{Q}$ and $\mathrm{R}$ as shown in table 5.1, the performance of Extended Kalman filter is shown is figures below:

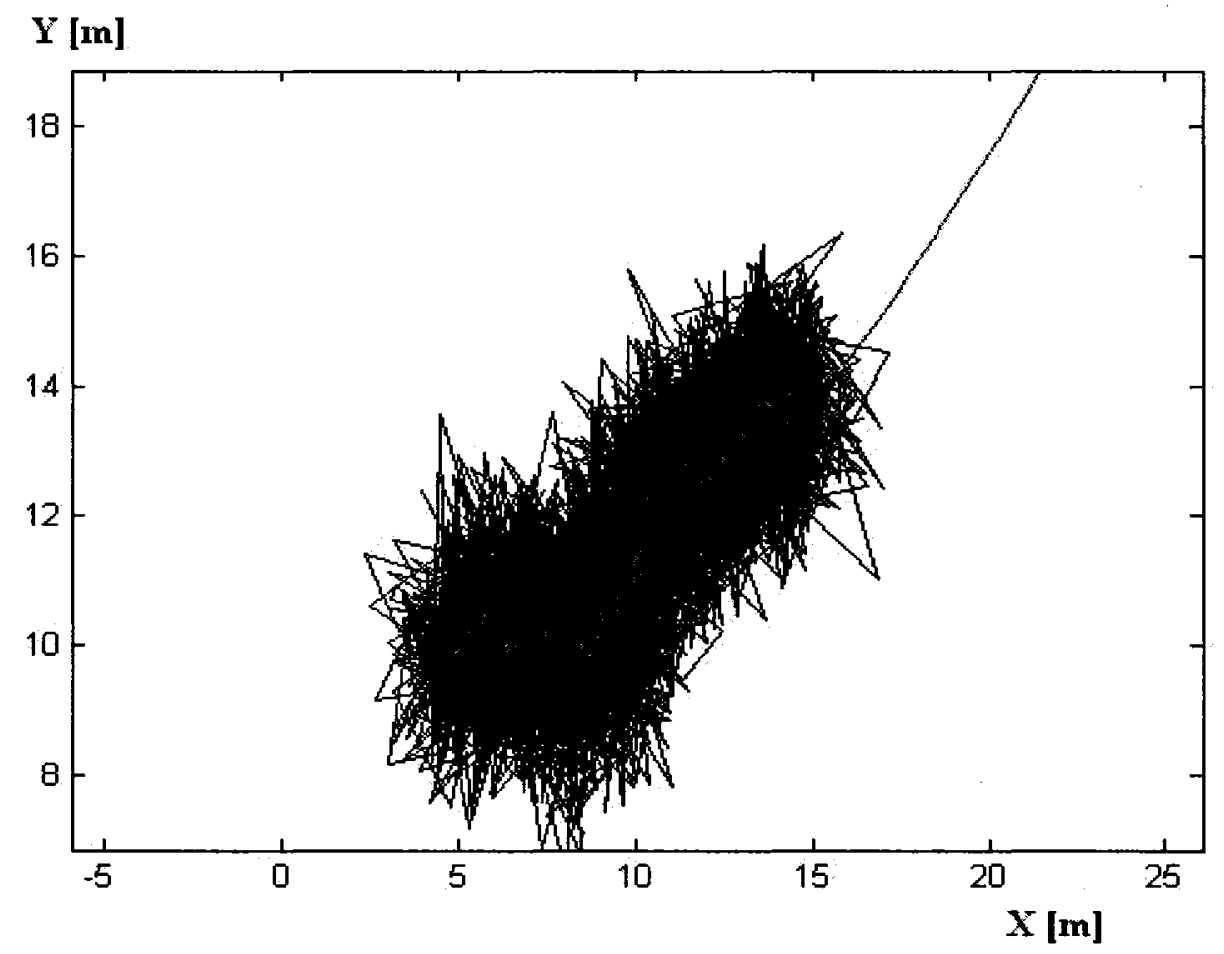

Figure 7.3: Updated location with $Q=1 I_{3} R=50 I_{3}$ 


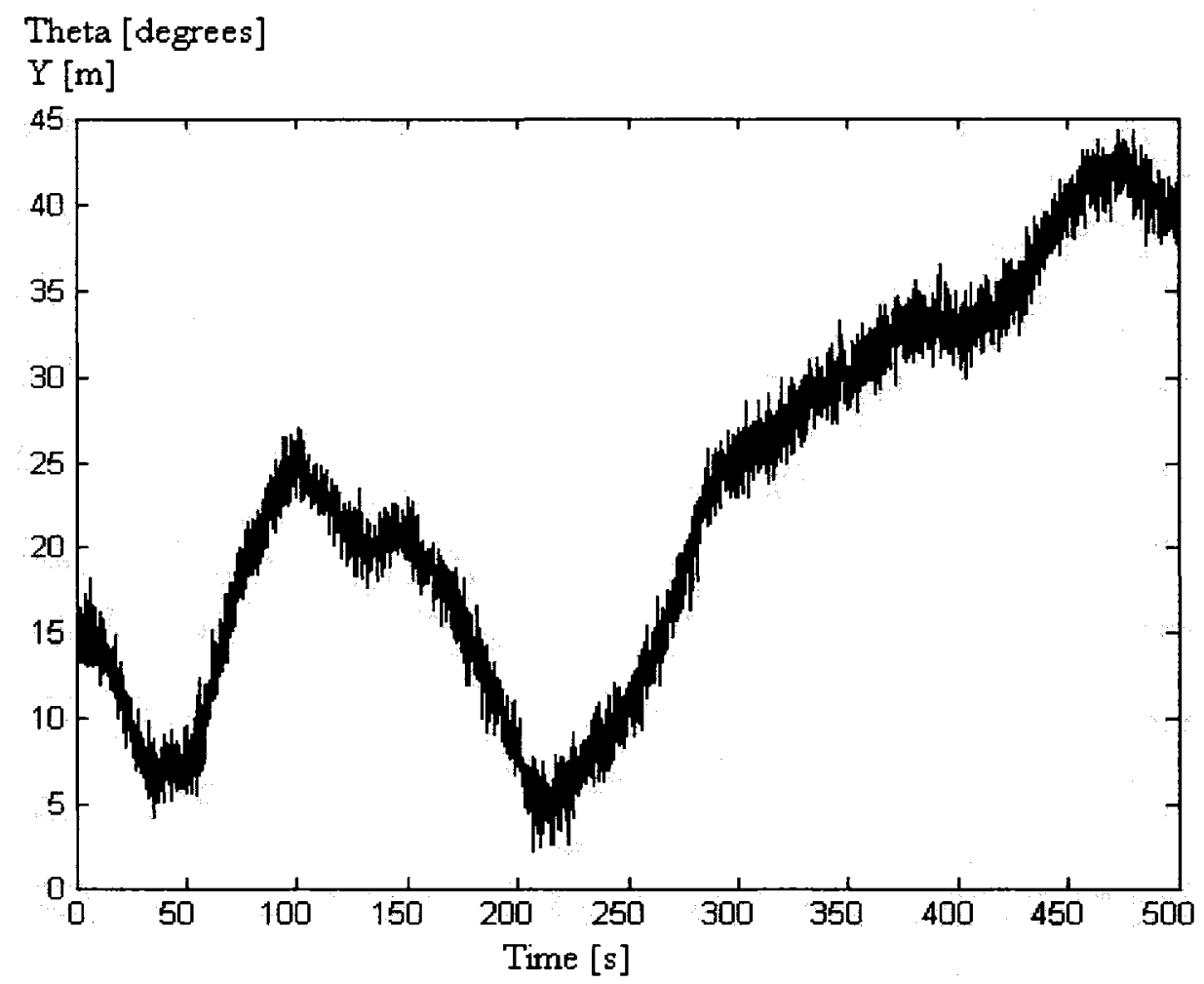

Figure 7.4: Updated orientation with $Q=1 I_{3} R=50 I_{3}$

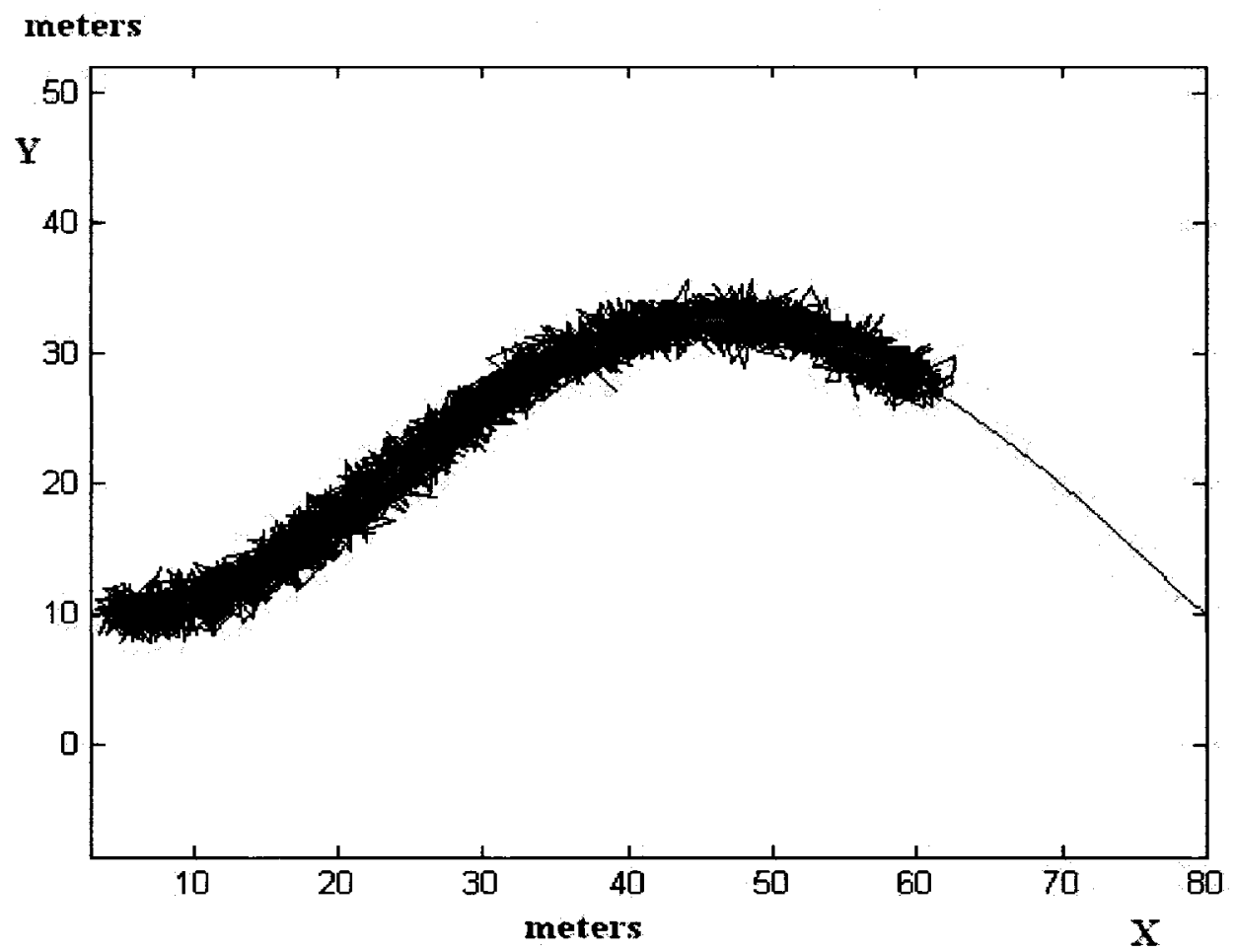

Figure 7.5: Updated location with $Q=1 I_{3} R=200 I_{3}$ 


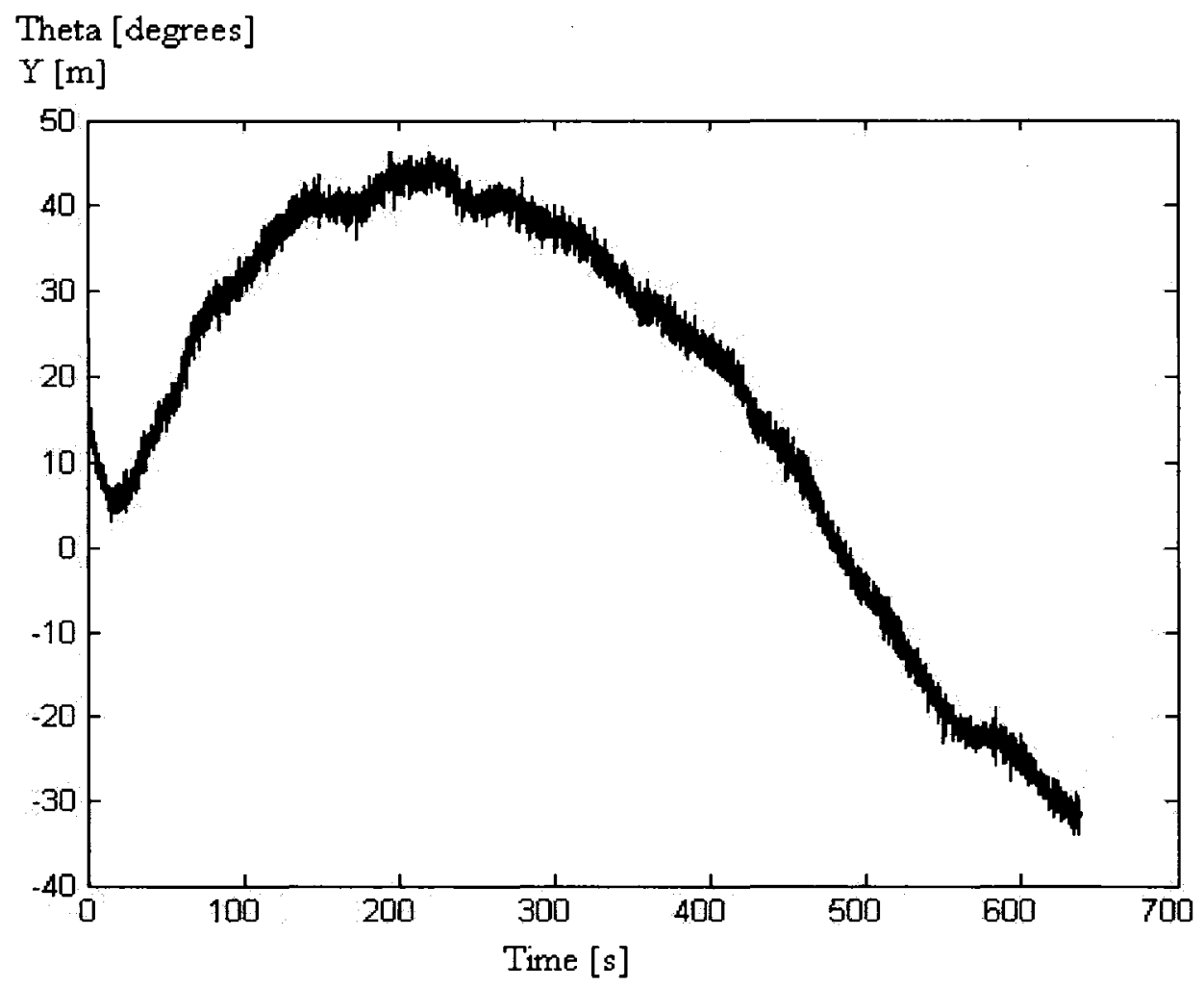

Figure 7.6: Updated orientation with $Q=1 I_{3} R=200 I_{3}$

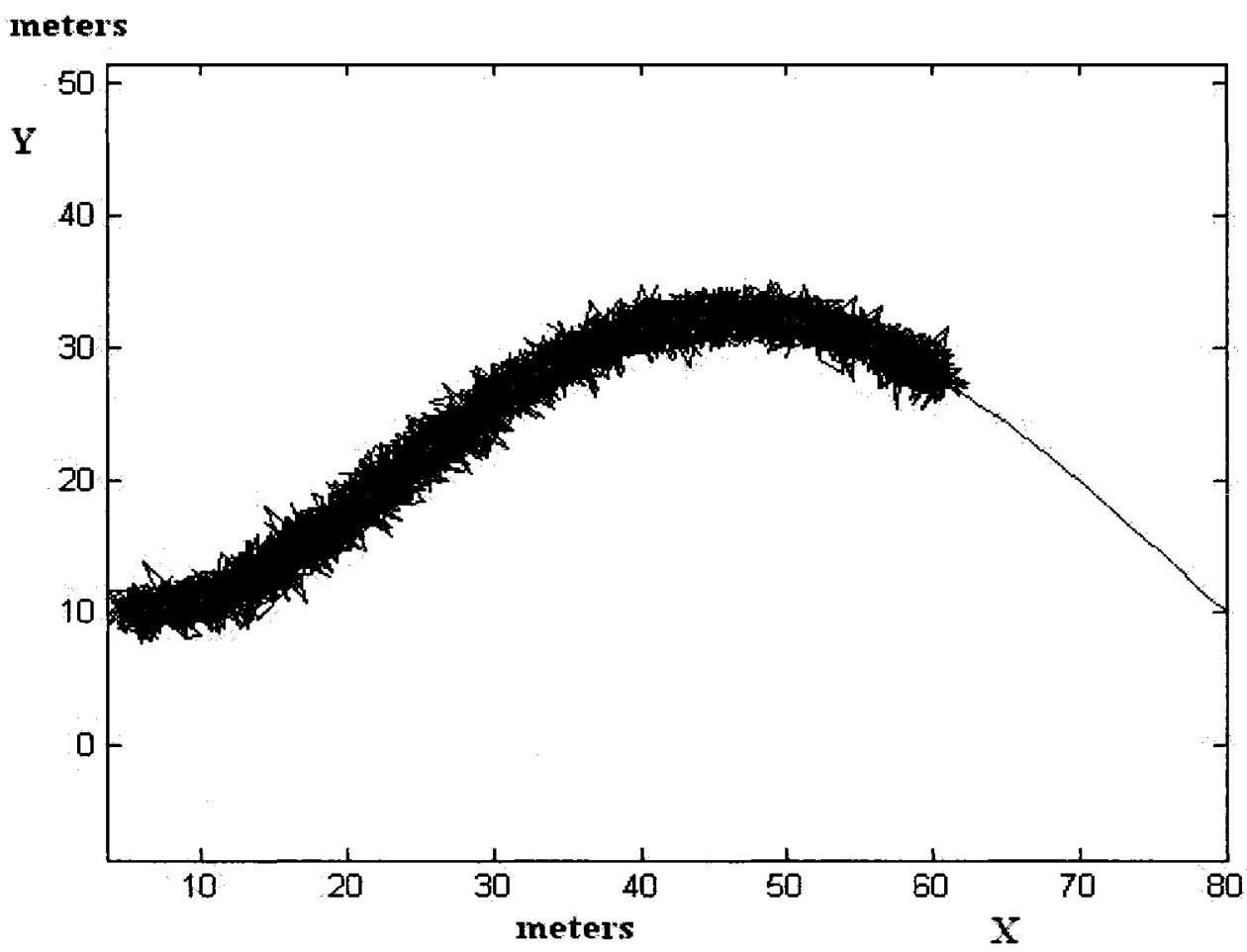

Figure 7.7: Updated location with $Q=1 I_{3} R=500 I_{3}$ 


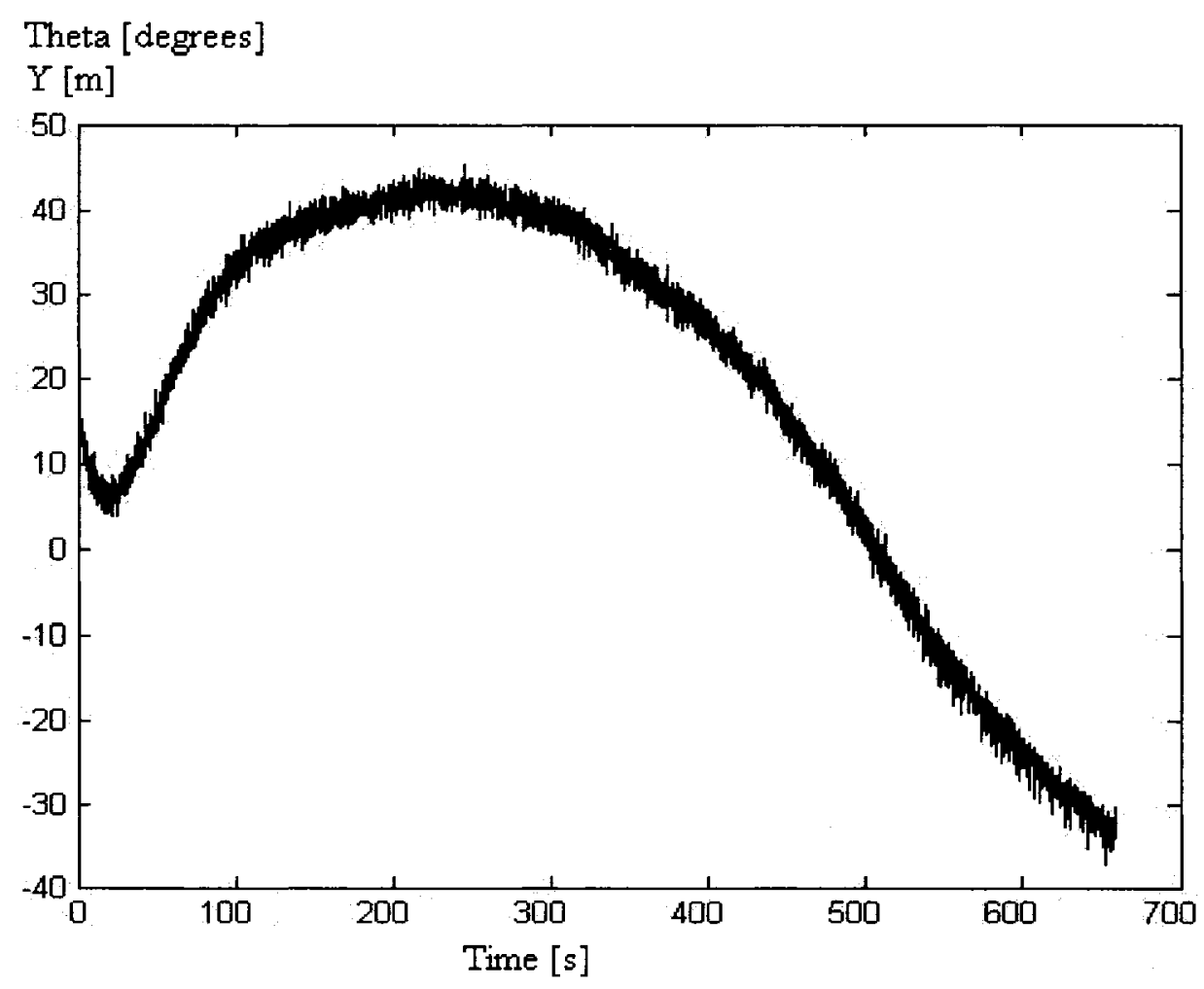

Figure 7.8: Updated orientation with $Q=1 I_{3} R=500 I_{3}$

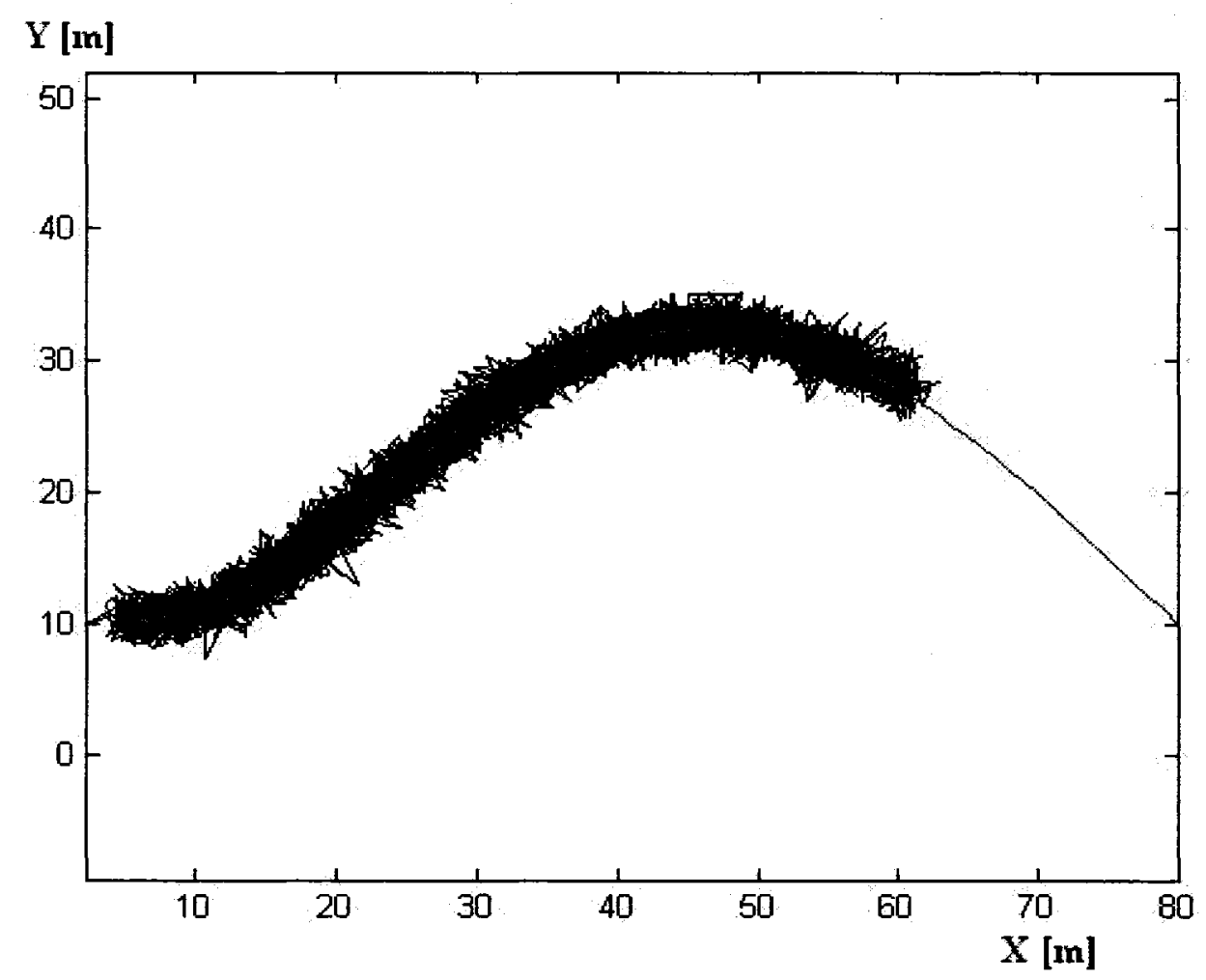

Figure 7.9: Updated location with $Q=1 I_{3} R=700 I_{3}$ 


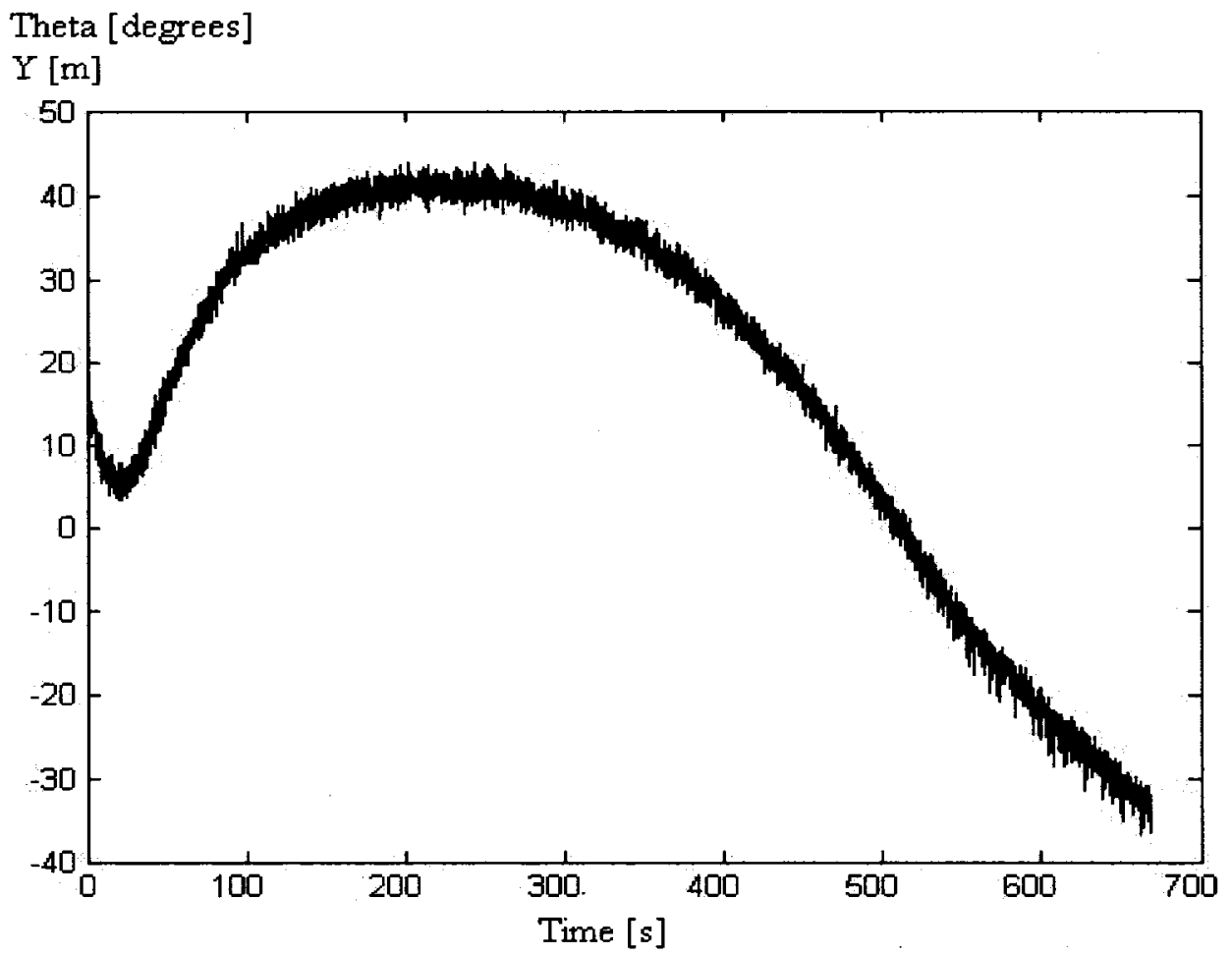

Figure 7.10: Updated orientation with $Q=1 I_{3} R=700 I_{3}$

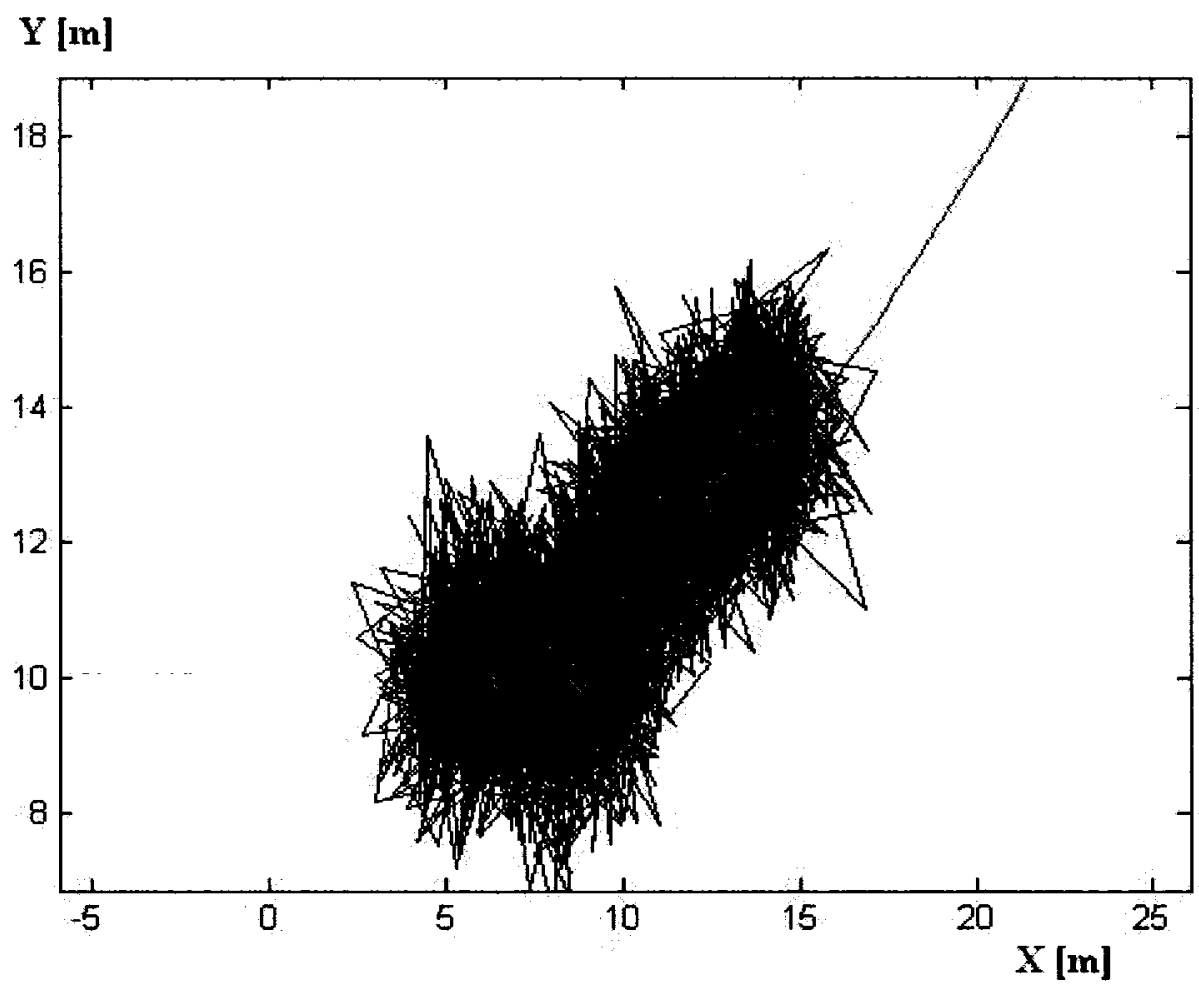

Figure 7.11: Updated location with $Q=10 I_{3} R=50 I_{3}$ 
Theta [degrees]

$\mathrm{Y}[\mathrm{m}]$

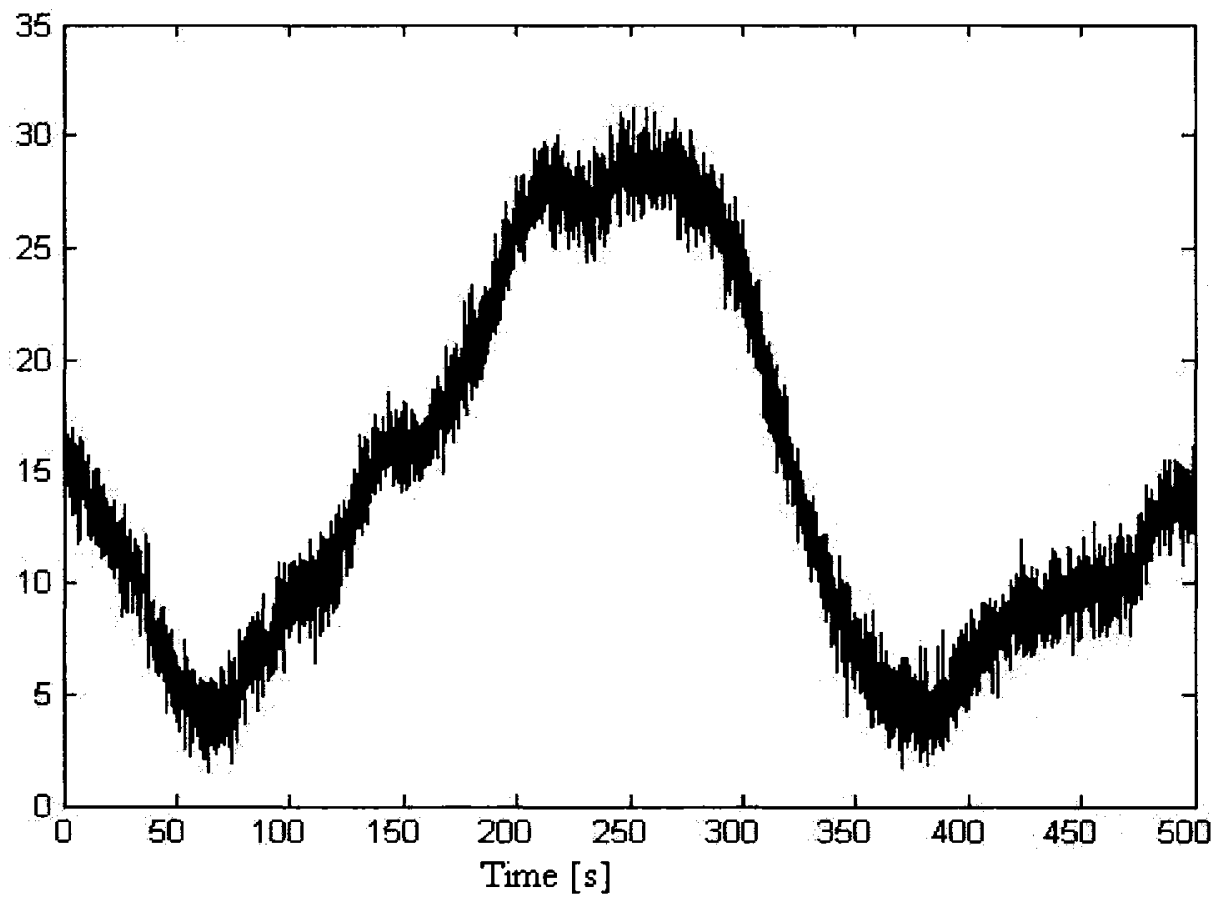

Figure 7.12: Updated orientation with $Q=10 I_{3} R=50 I_{3}$

$\mathrm{Y}[\mathrm{m}]$

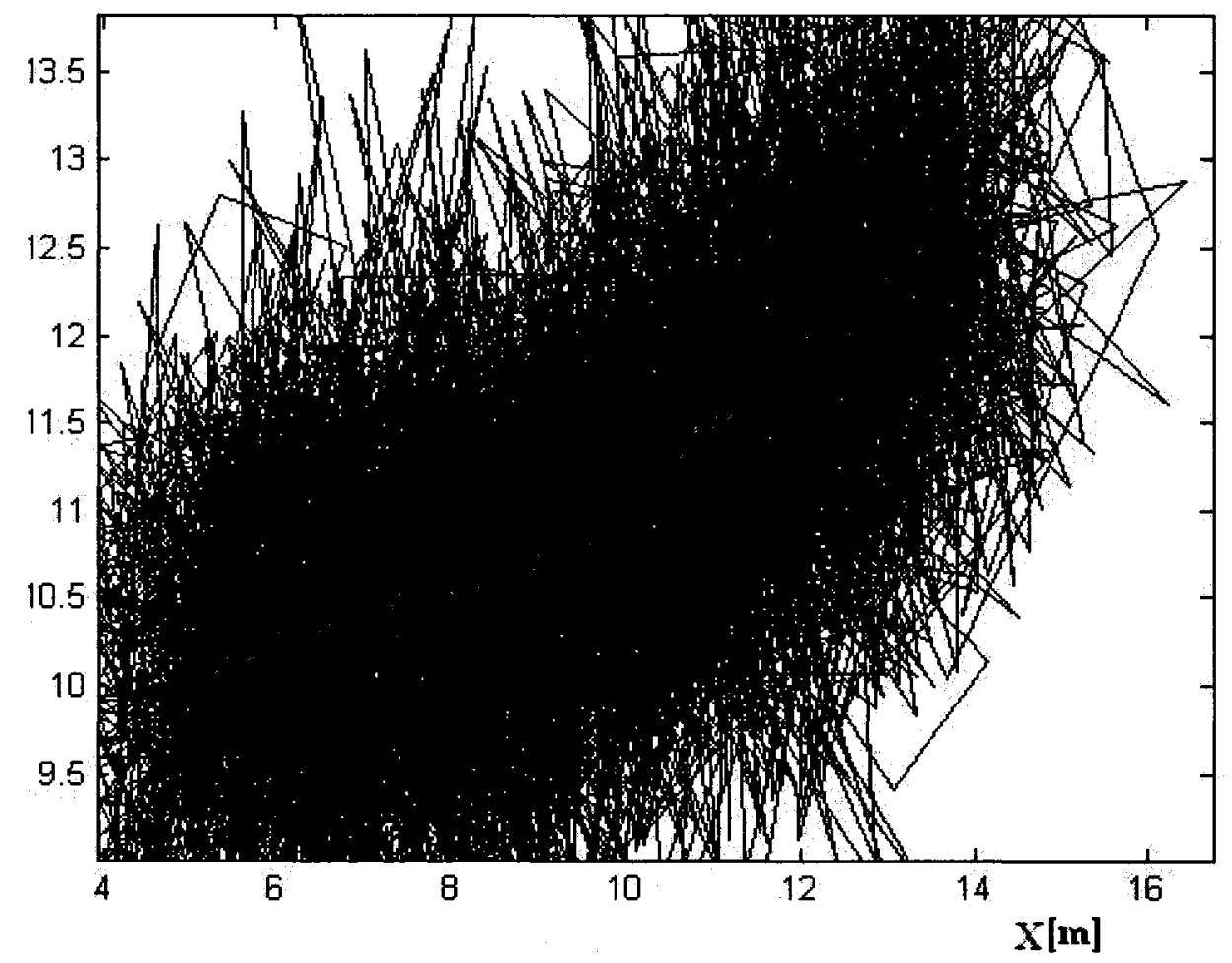

Figure 7.13: Updated location with $Q=10 I_{3} R=200 I_{3}$ 


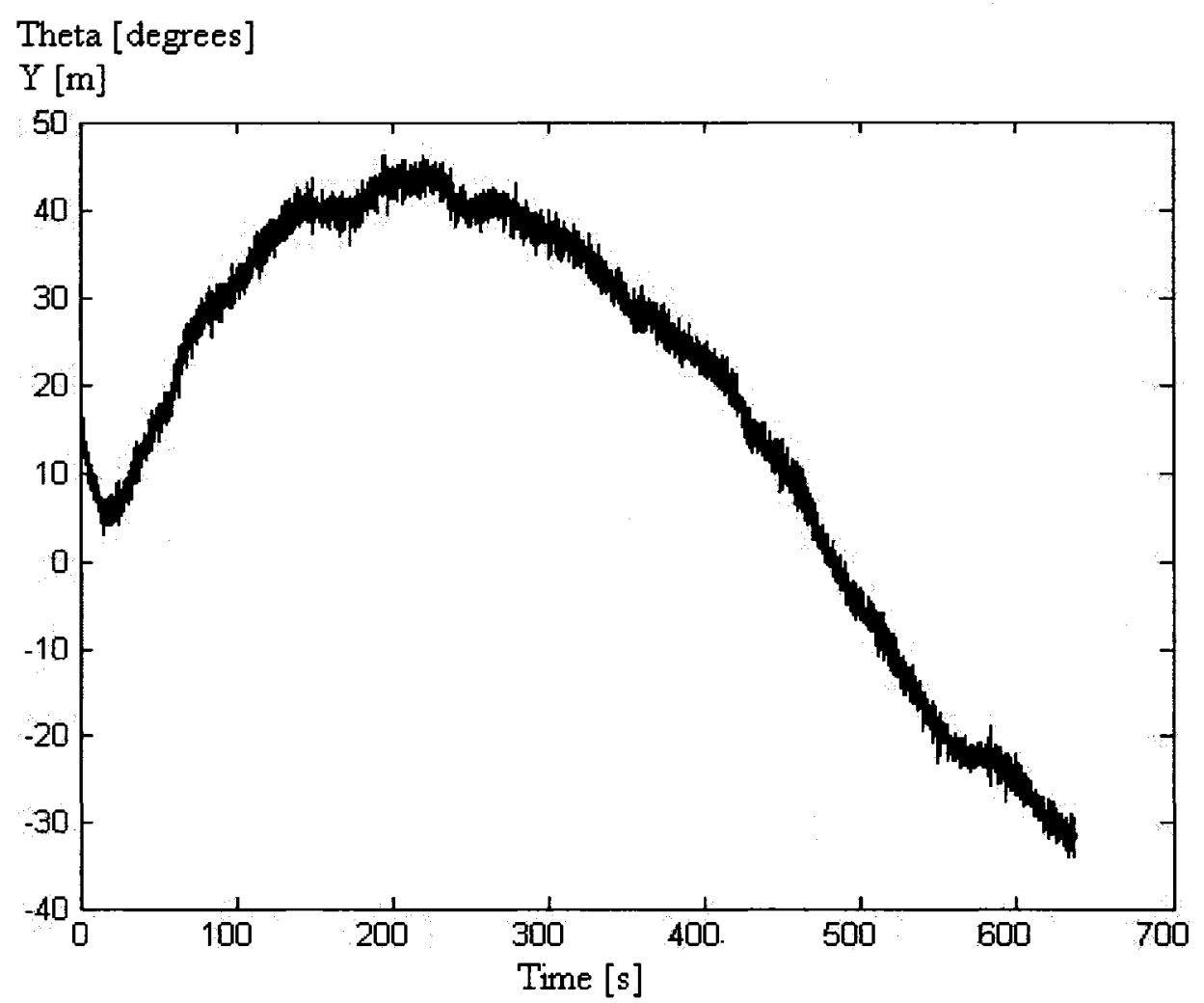

Figure 7.14: Updated orientation with $Q=10 I_{3} R=200 I_{3}$

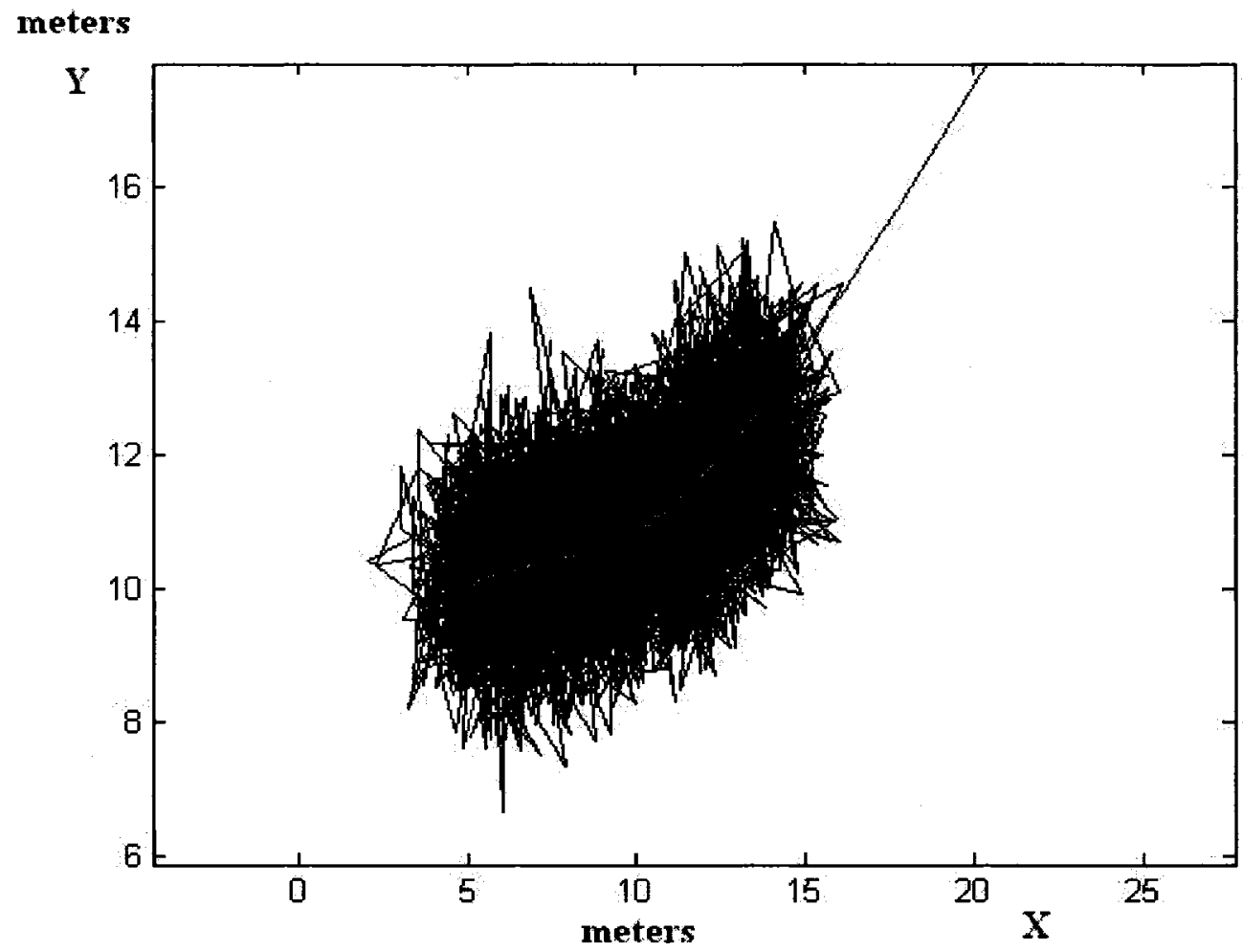

Figure 7.15: Updated location with $Q=10 I_{3} R=500 I_{3}$ 


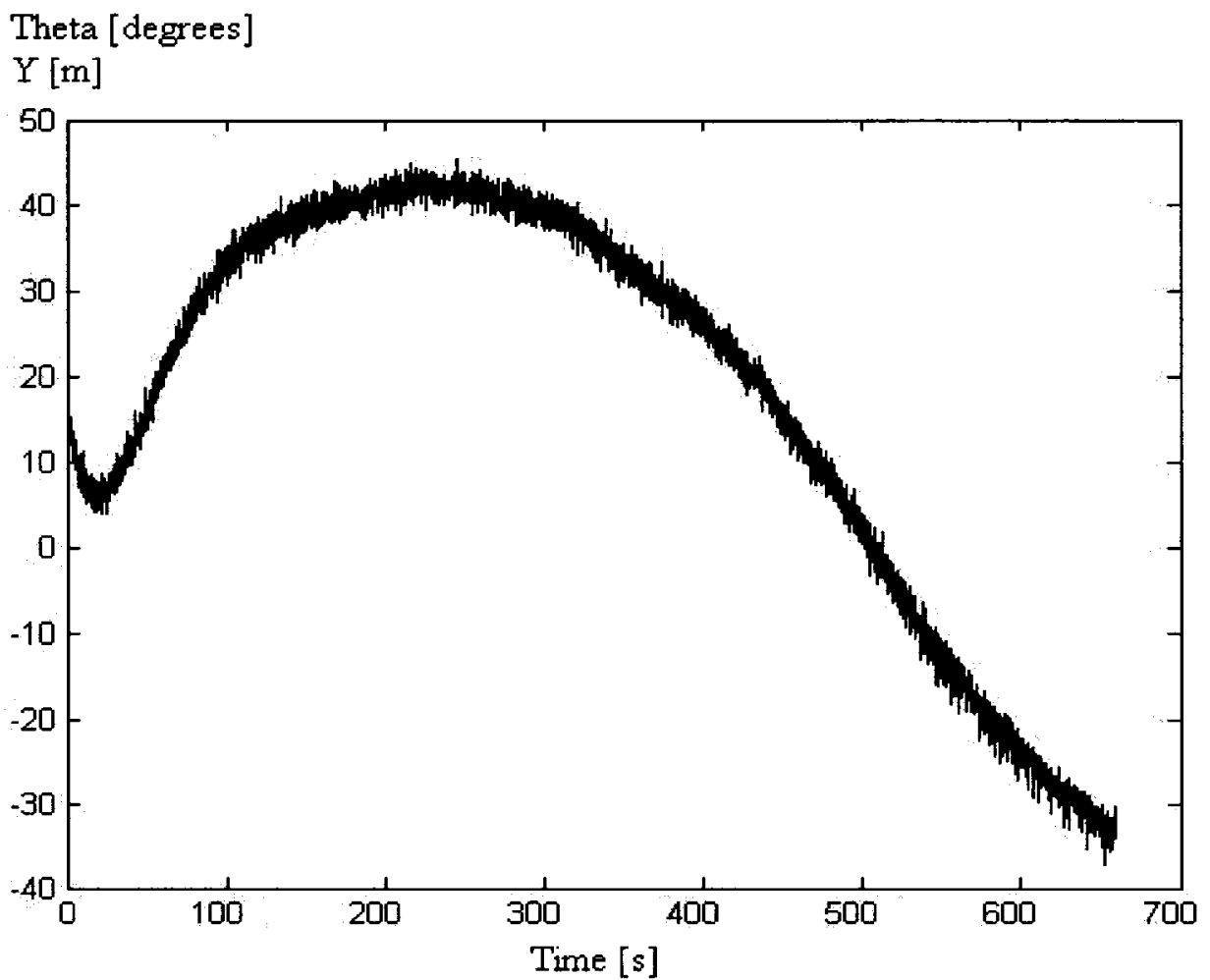

Figure 7.16: Updated orientation with $Q=10 I_{3} R=500 I_{3}$

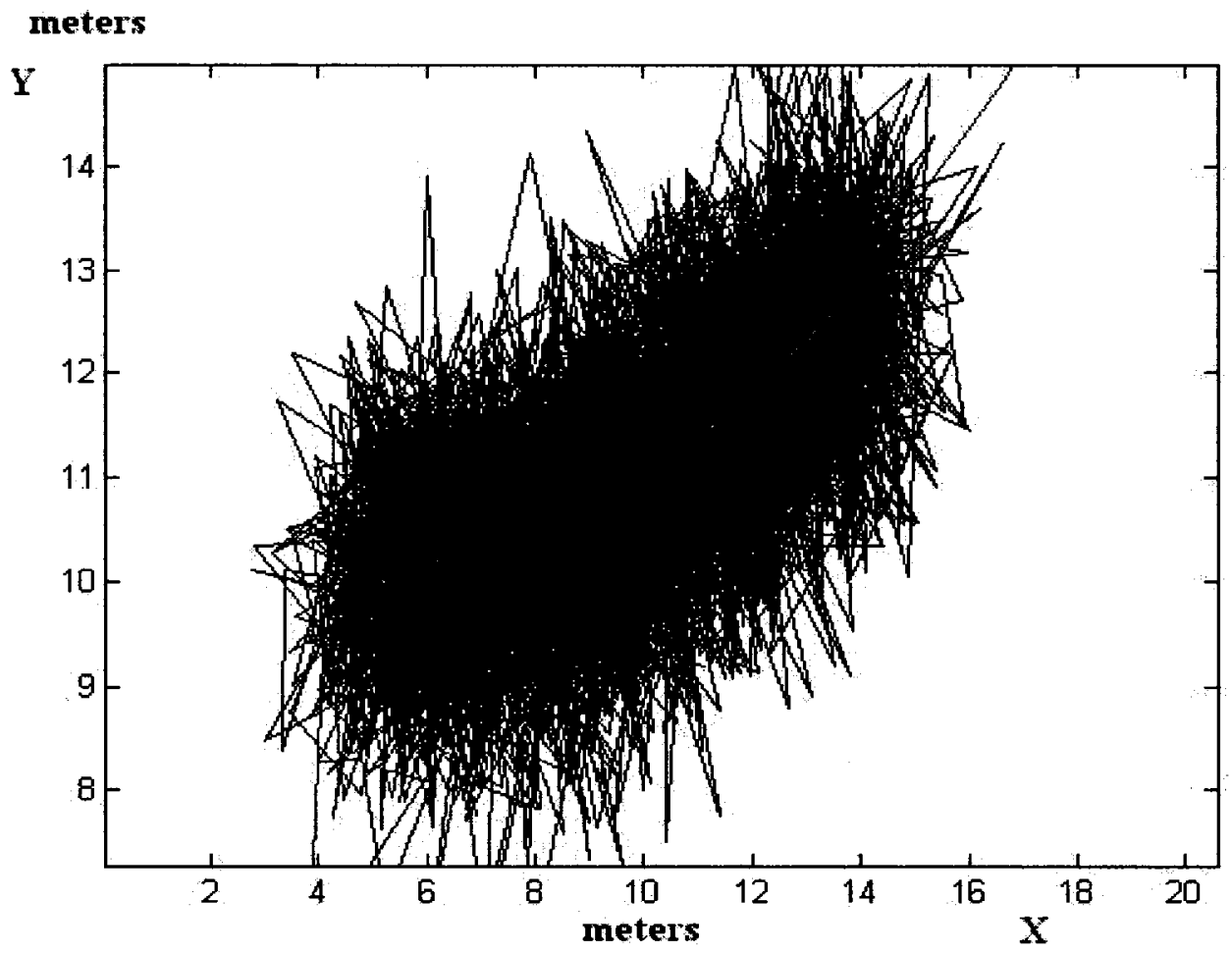

Figure 7.17: Updated location with $Q=10 I_{3} R=700 I_{3}$ 


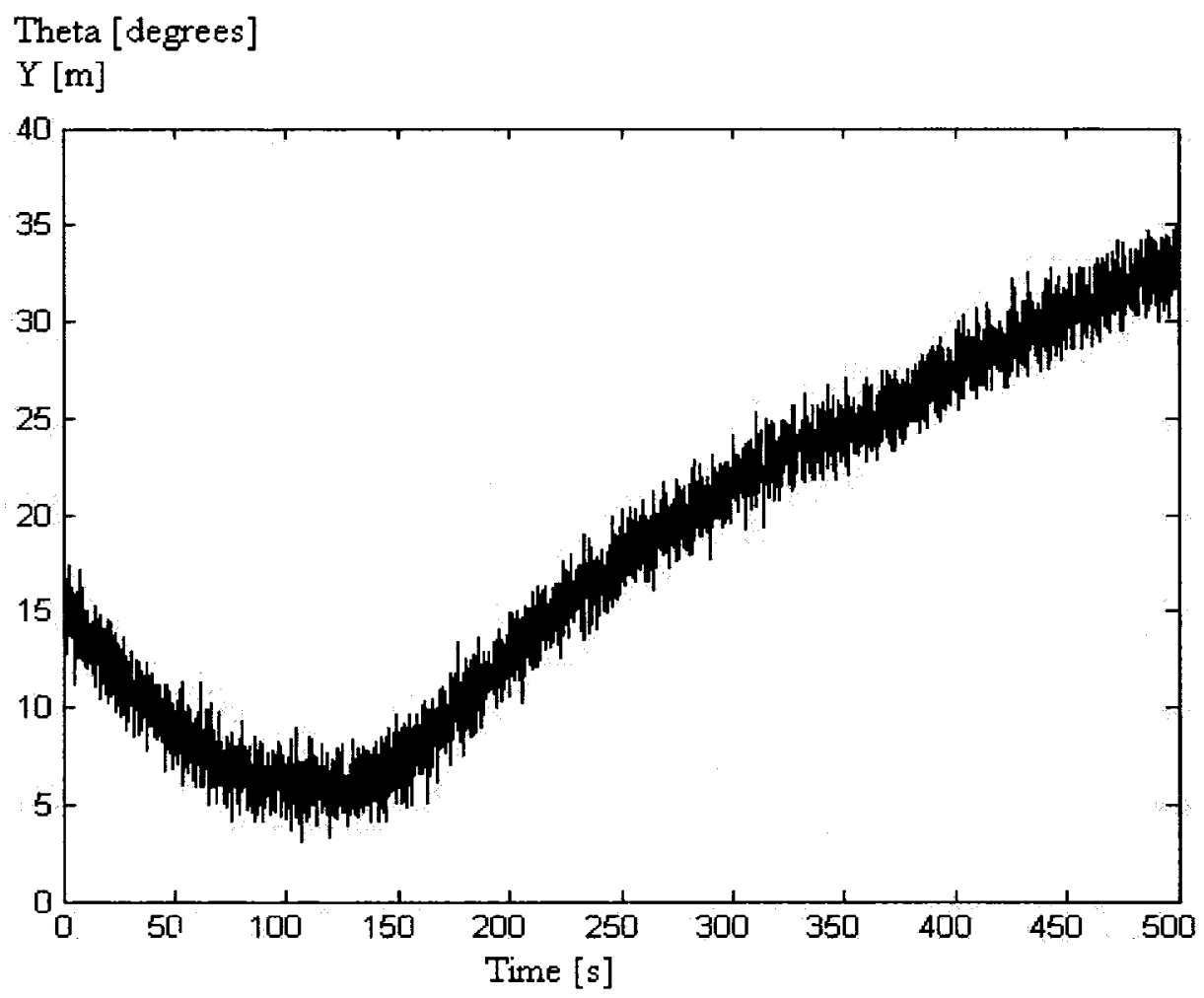

Figure 7.18: Updated orientation with $Q=10 I_{3} R=700 I_{3}$

From these results we can observer that as value of $\mathrm{R}$ increases, the updated states come very close to predicted states that means that Kalman filter relies less on sensor results. The noise is Gaussian with zero mean and unit variance. The difference in predicted value and one that is obtained from sensors is considered as an error and is already shown in chapter 3 where we also discussed about our selection. The updated values for the states predicted in example of previous section using EKF with $Q=10 . I_{3}$ and $R=500 . I_{3}$ are: $x=7.9739, y=21.9876, \theta=45$. 


\subsubsection{Trajectory tracking}

As discussed in chapter 5, path tracking is a problem of finding gains of two controllers so as to minimize the offsets. The two controllers are steering controller that generates necessary gains to minimize orientation, distance/lateral and steering angle offsets and propulsion or velocity controller generates gains to minimize velocity offsets. The steering controller generates gains using LQR which in turn depends on values of matrices $\mathbf{Q}$ and $\mathbf{R}$.

For the car-like mobile robot moving without slippage, the task of controlling the steering input is equivalent to controlling of the linear system as shown by equation (5.44) and the error dynamics matrix $\mathrm{A}$ is given by equation (5.45).

Consider, for instance, the desired steering angle required for next step is 10 degrees, length $\mathrm{L}$ and wheel base $\mathbf{B}$ of robot is 1.5 meters and 1 meter respectively as in this work and if the desired longitudinal velocity is 1 meter per second then above $\mathbf{A}$ matrix is given as (5.55):

$$
\mathbf{A}=\left(\begin{array}{ccc}
0 & 0 & 0.6873 \\
1 & 0 & 0.6873 \\
0 & 0 & 0
\end{array}\right) \text { and } \mathbf{B}=\left(\begin{array}{l}
0 \\
0 \\
1
\end{array}\right)
$$

In all cases the vehicle is moving with the speed of 1 meter per second speed which is equal to 3.6 kilometers per hour. Hence, the angular velocity is restricted to $0.67 \mathrm{rad} / \mathrm{sec}$. Moreover, the vehicle satisfies all kinematic constraints imposed on it as described in chapter 2. The controller gains with above matrix $\mathbf{A}, C_{1}=10$ and $C_{2}=1$, using Riccatti 
equation (5.48) and feedback control law $\mathbf{u}=-\mathbf{K}_{s} . \mathbf{x}$ are $\mathrm{k}_{1}=4.7111, \mathrm{~K}_{2}=3.1623, \mathrm{~K}_{3}=$ 4.5632. The performance of controller with parametric values shown in table 5.1 is below:

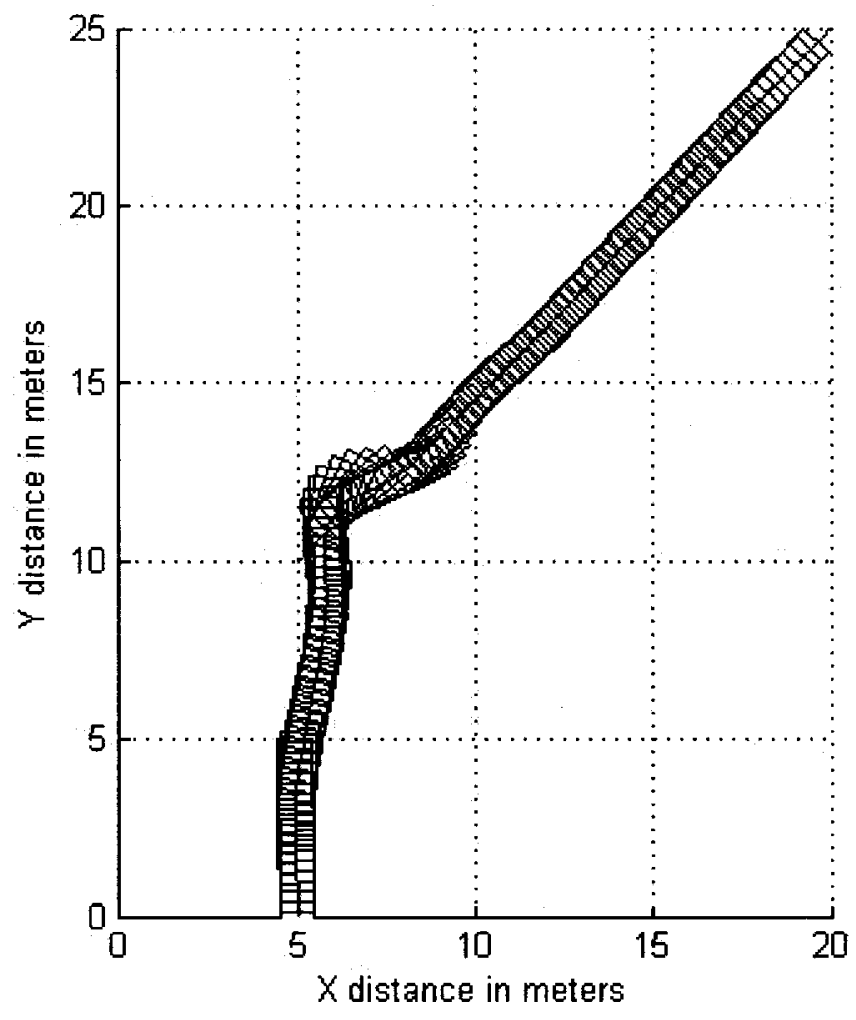

(a) 


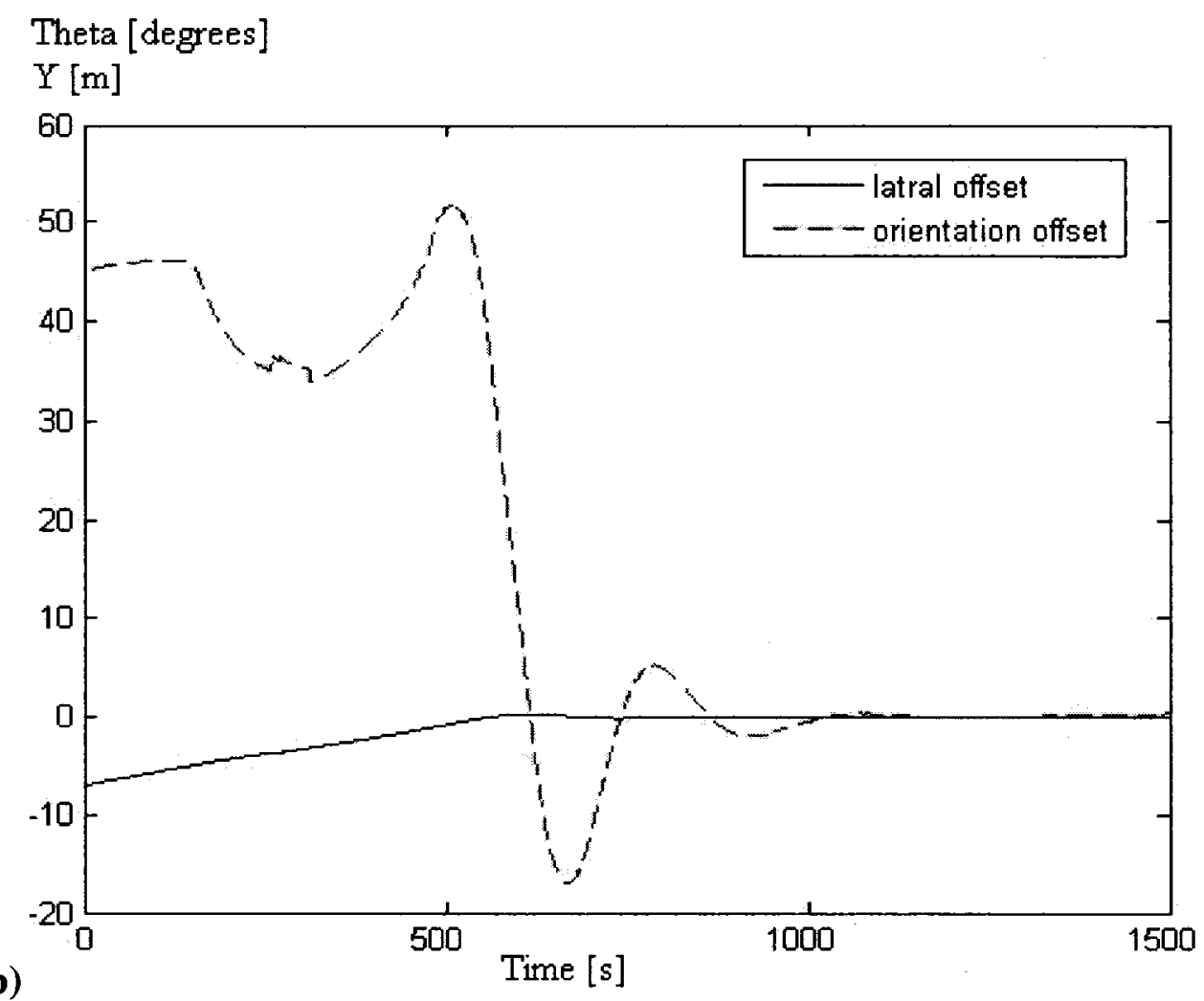

Figure 7.19: Trajectory tracking and offsets for $\mathrm{C}_{1}=1$ and $\mathrm{C}_{2}=50$.

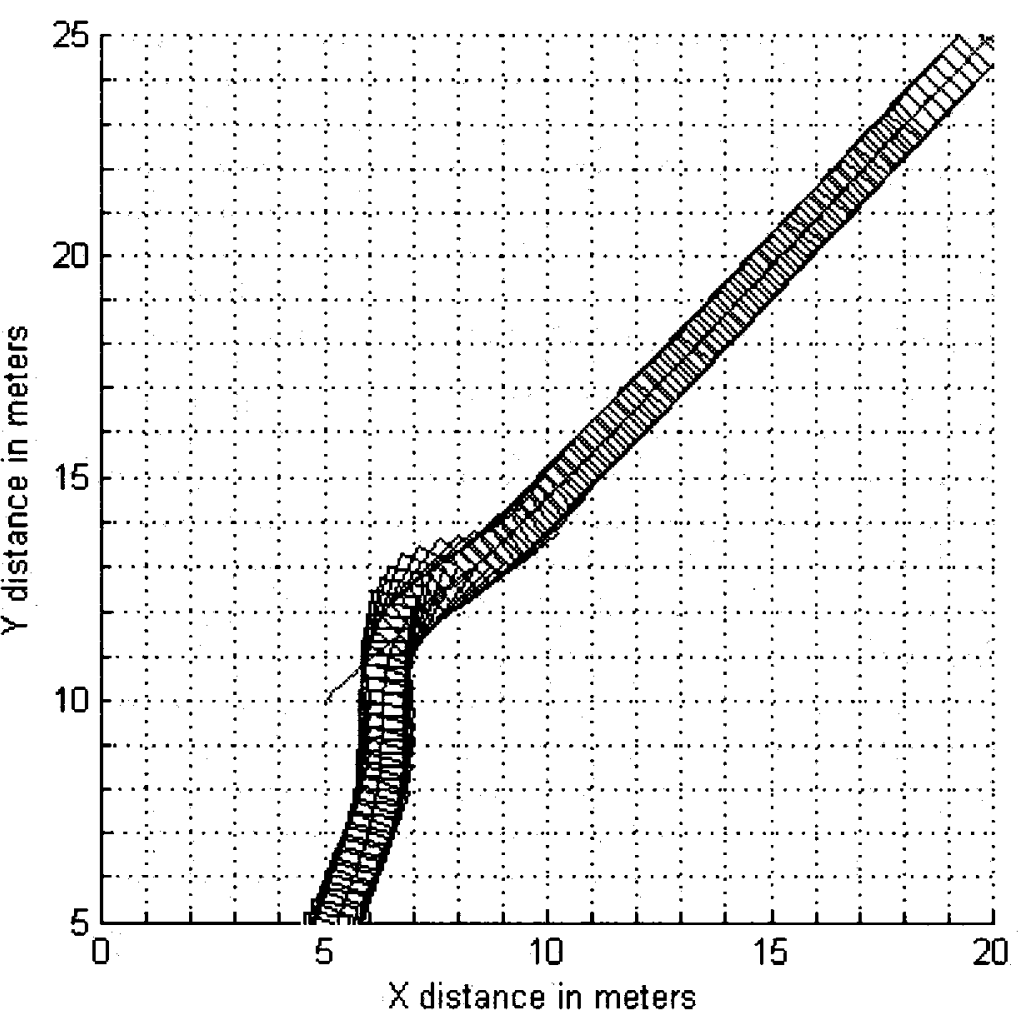




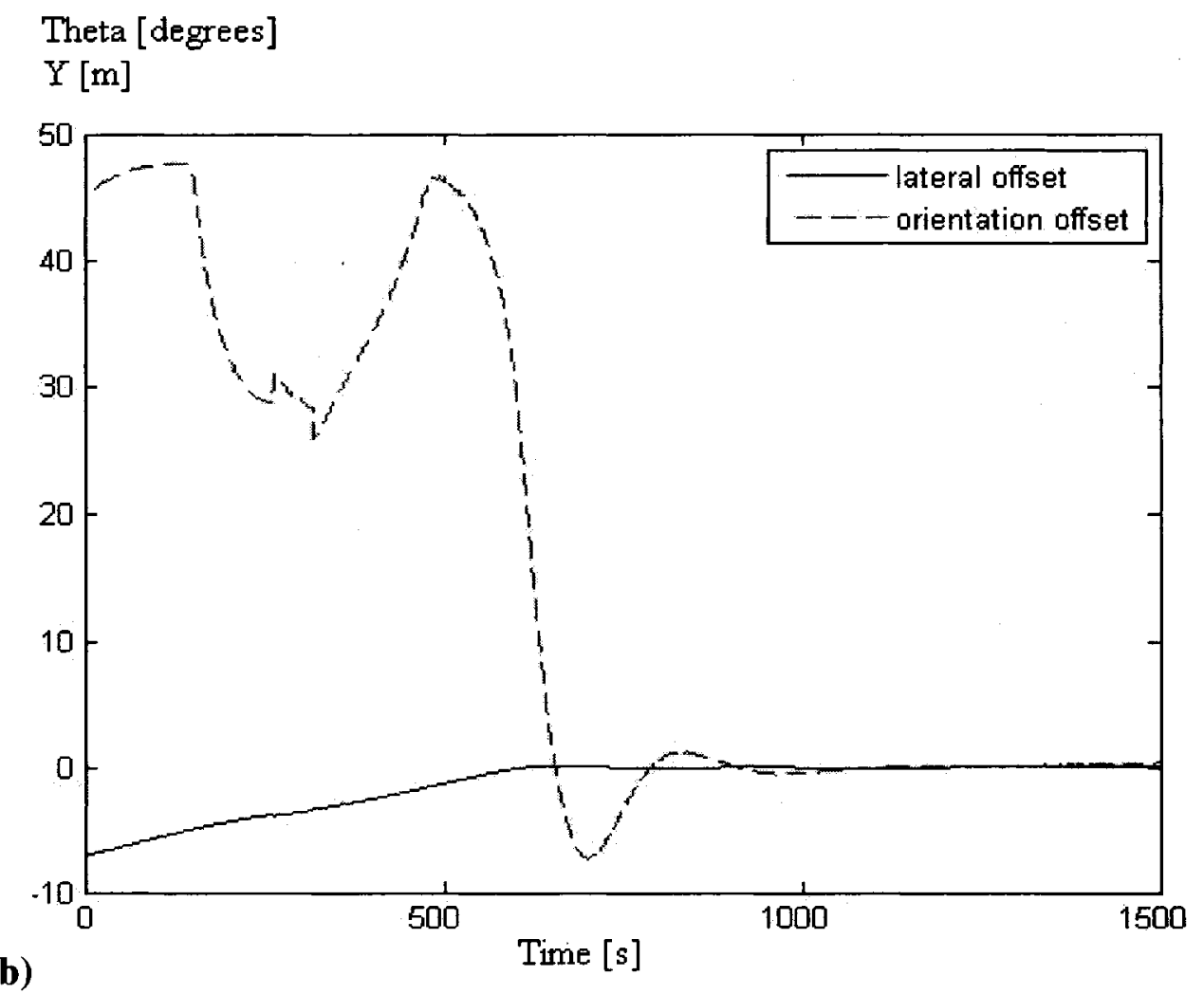

Figure 7.20: Trajectory tracking and offsets for $C_{1}=1$ and $C_{2}=1$.

(a)

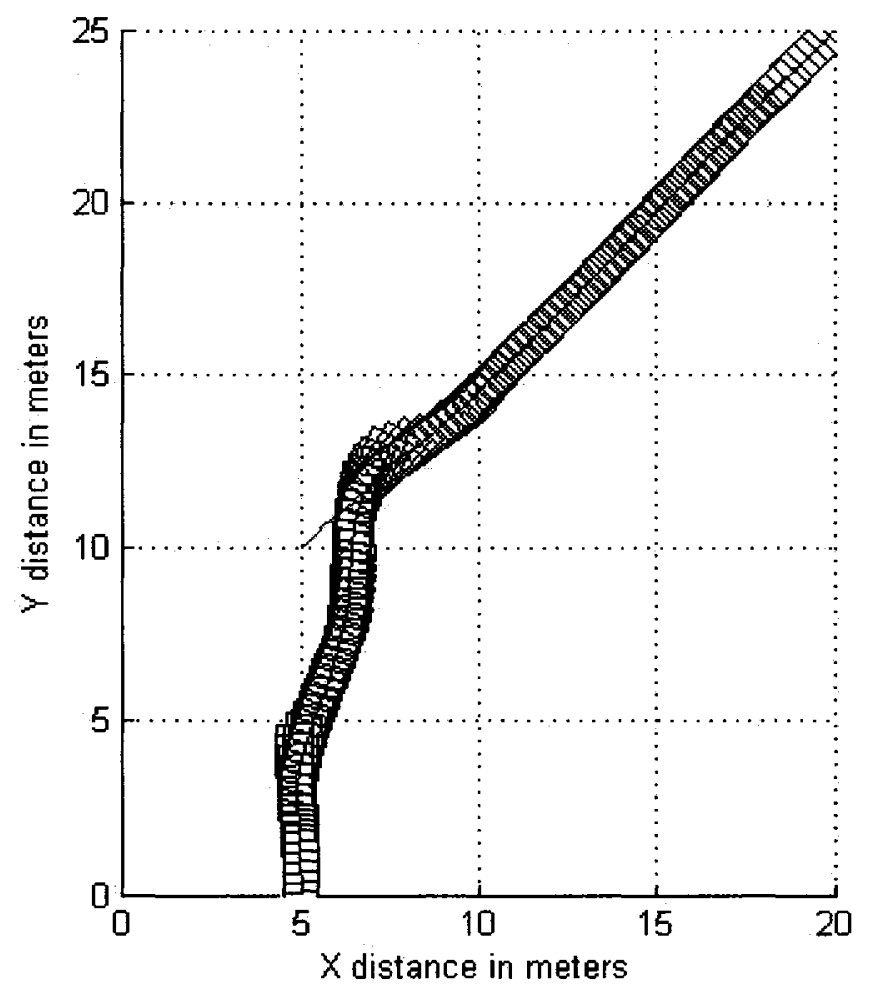




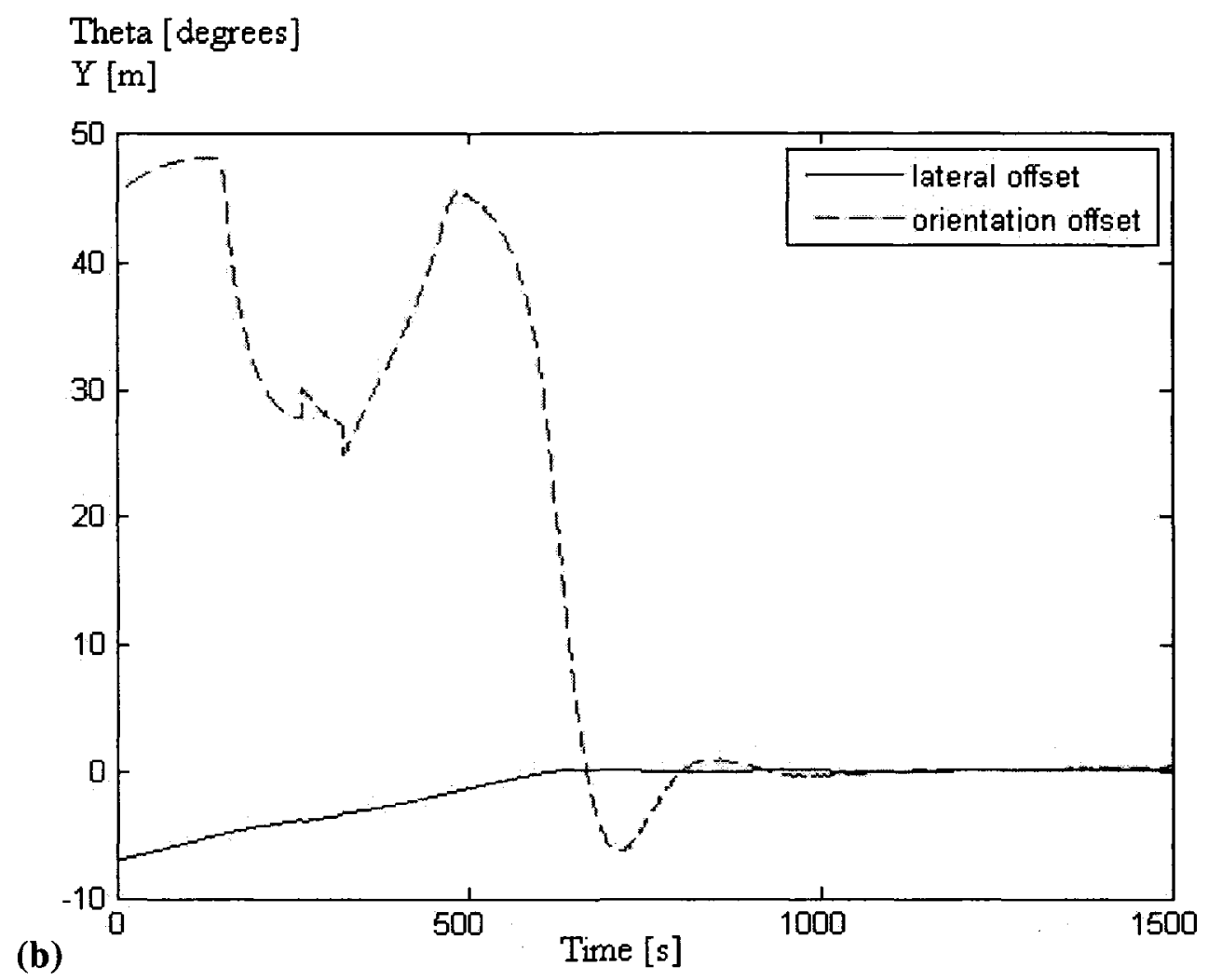

Figure 7.21: Trajectory tracking and offsets for $C_{1}=20$ and $C_{2}=10$.

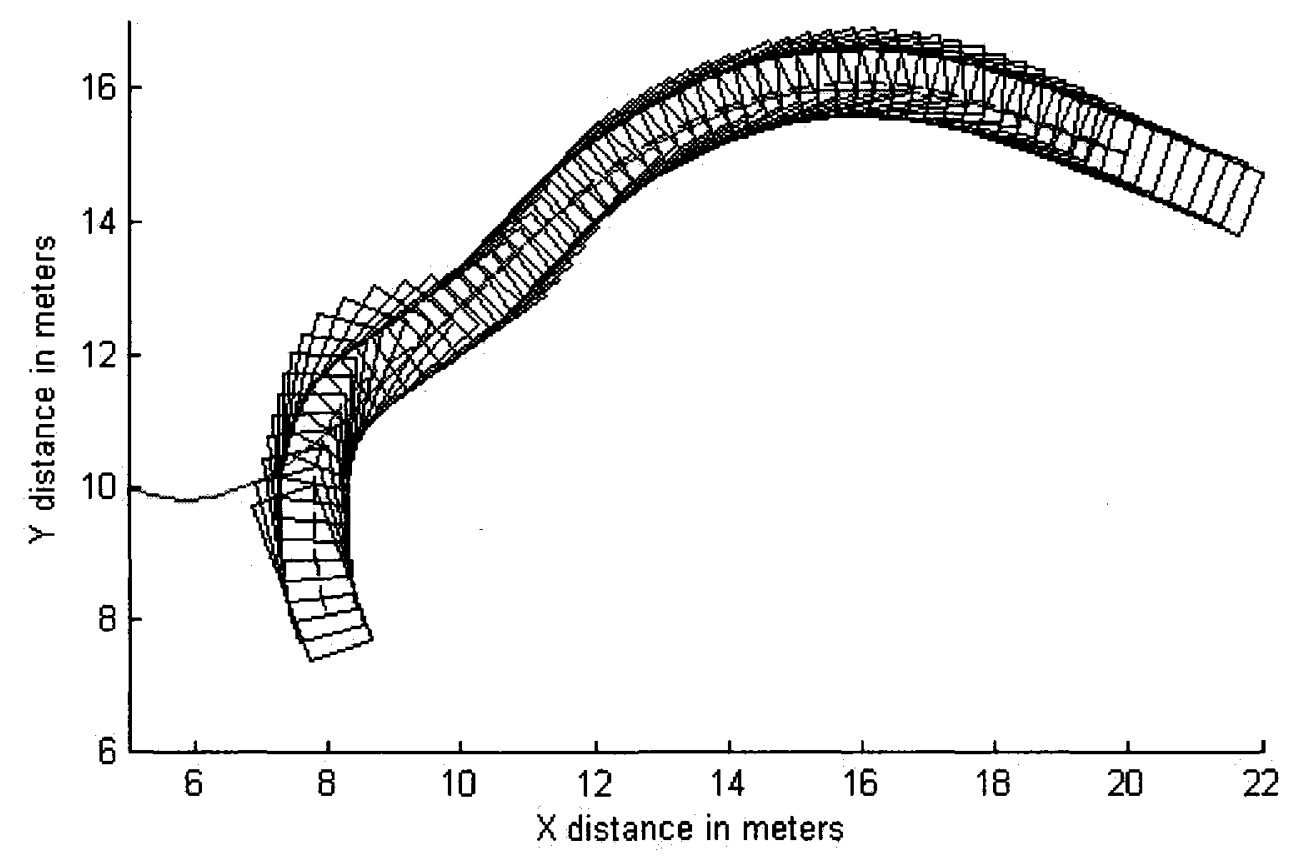

(a) 


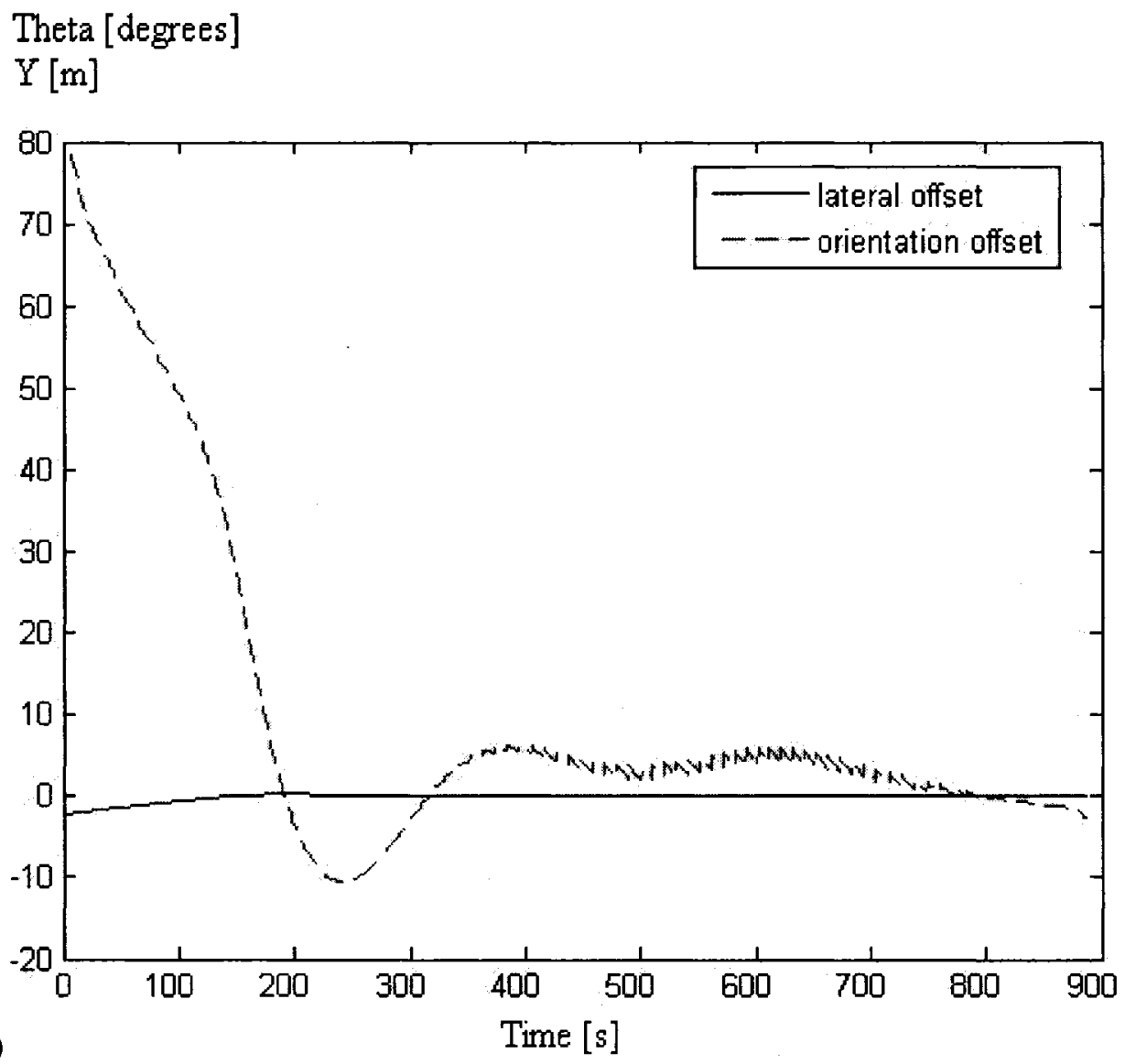

Figure 7.22: Trajectory tracking and offsets for curved path with $C_{1}=1$ and $C_{2}=50$.

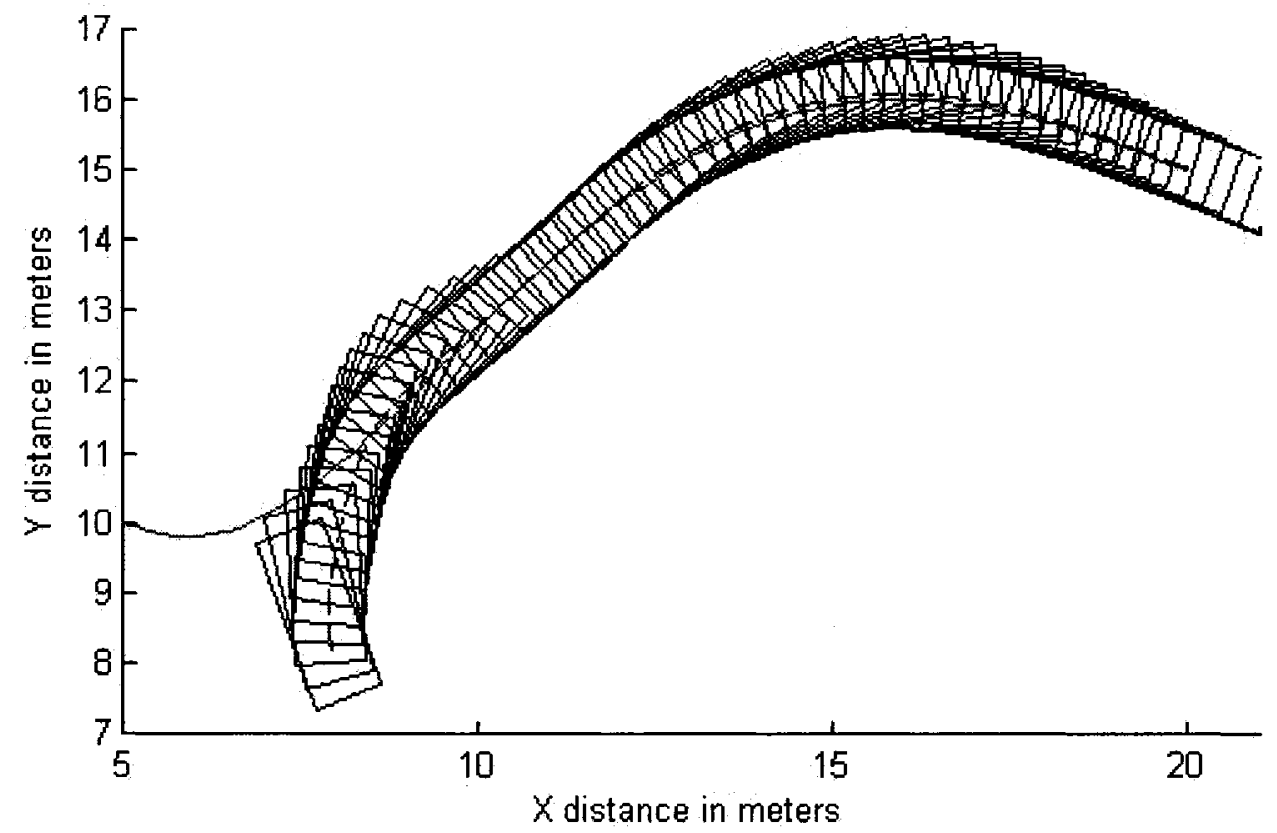

(a) 


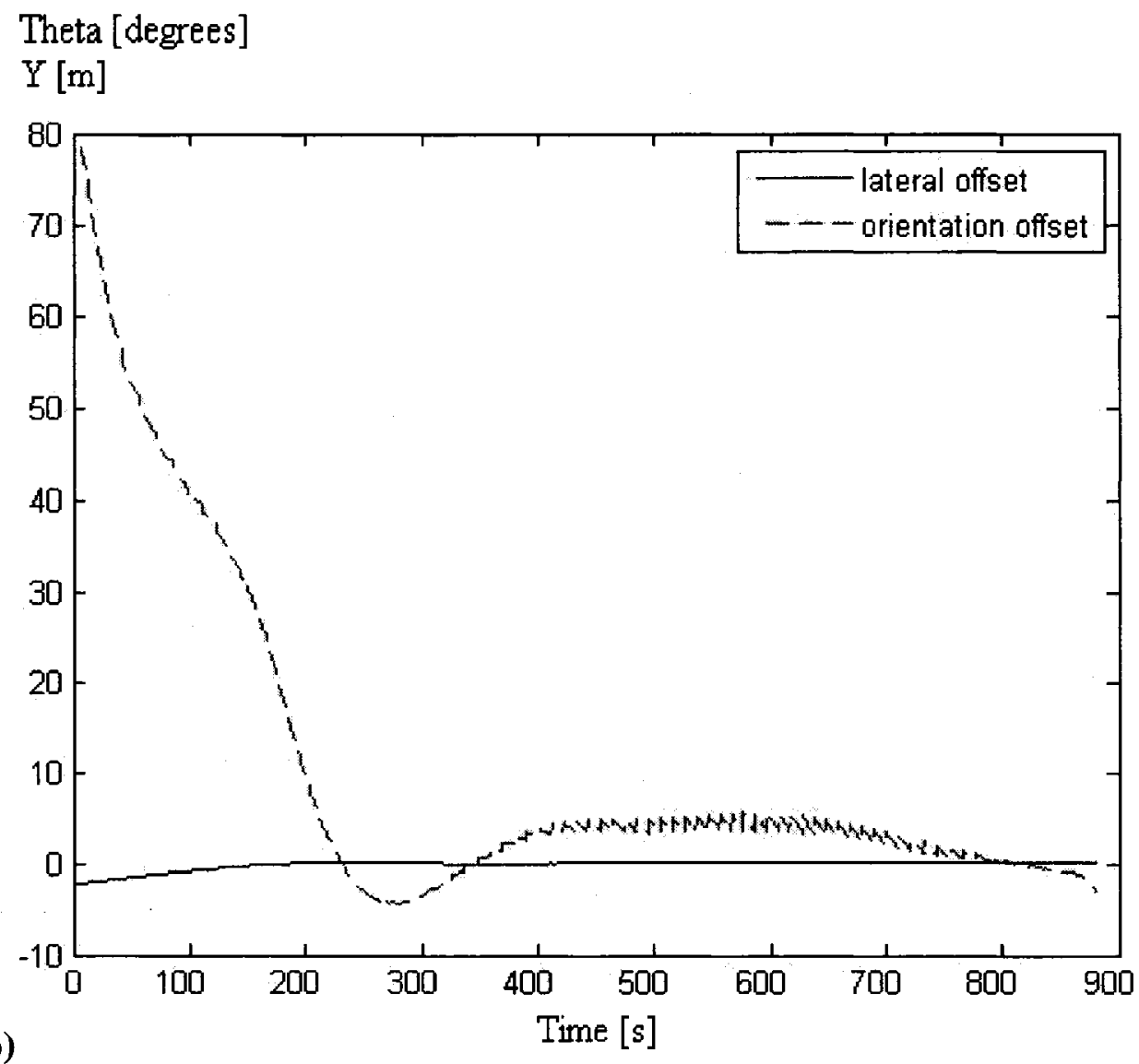

Figure 7.23: Trajectory tracking and offsets for curved path with $\mathrm{C}_{1}=1$ and $\mathrm{C}_{2}=1$.

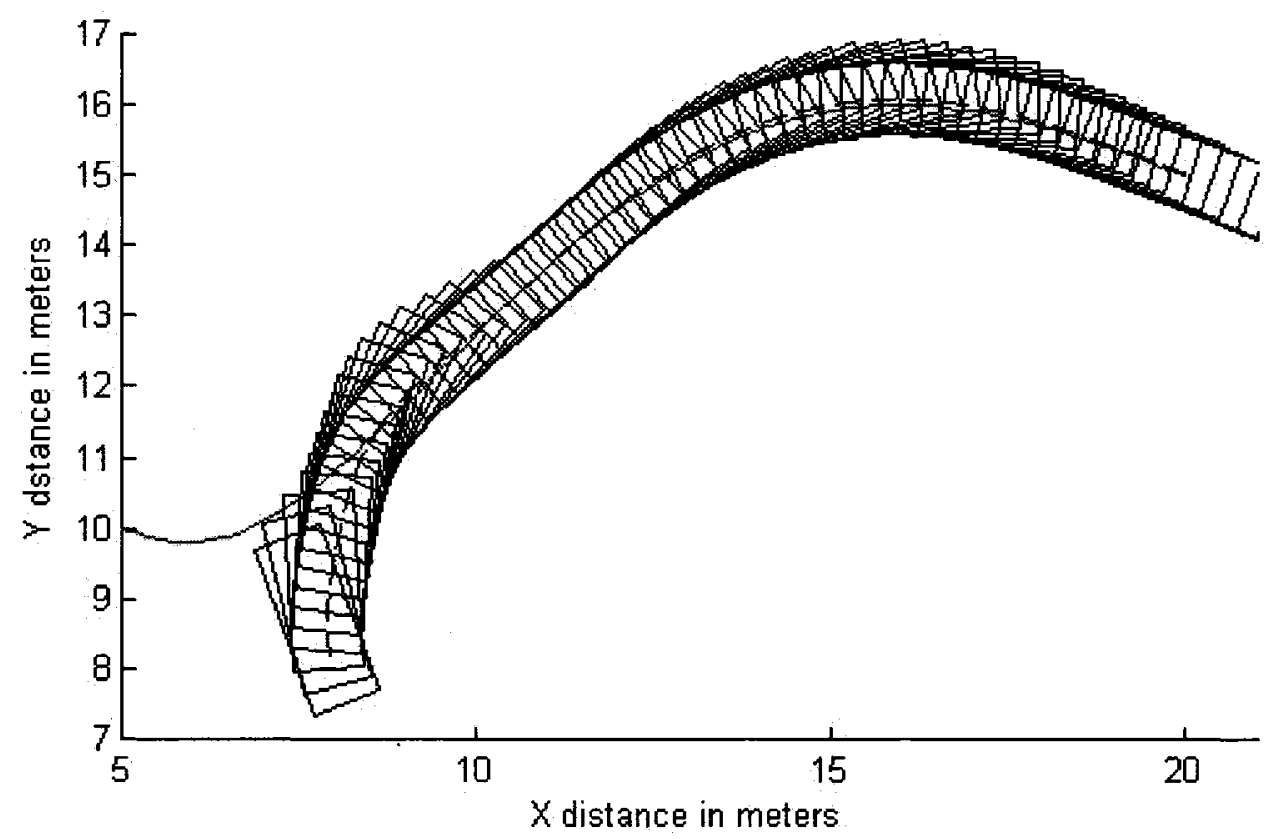

(a) 


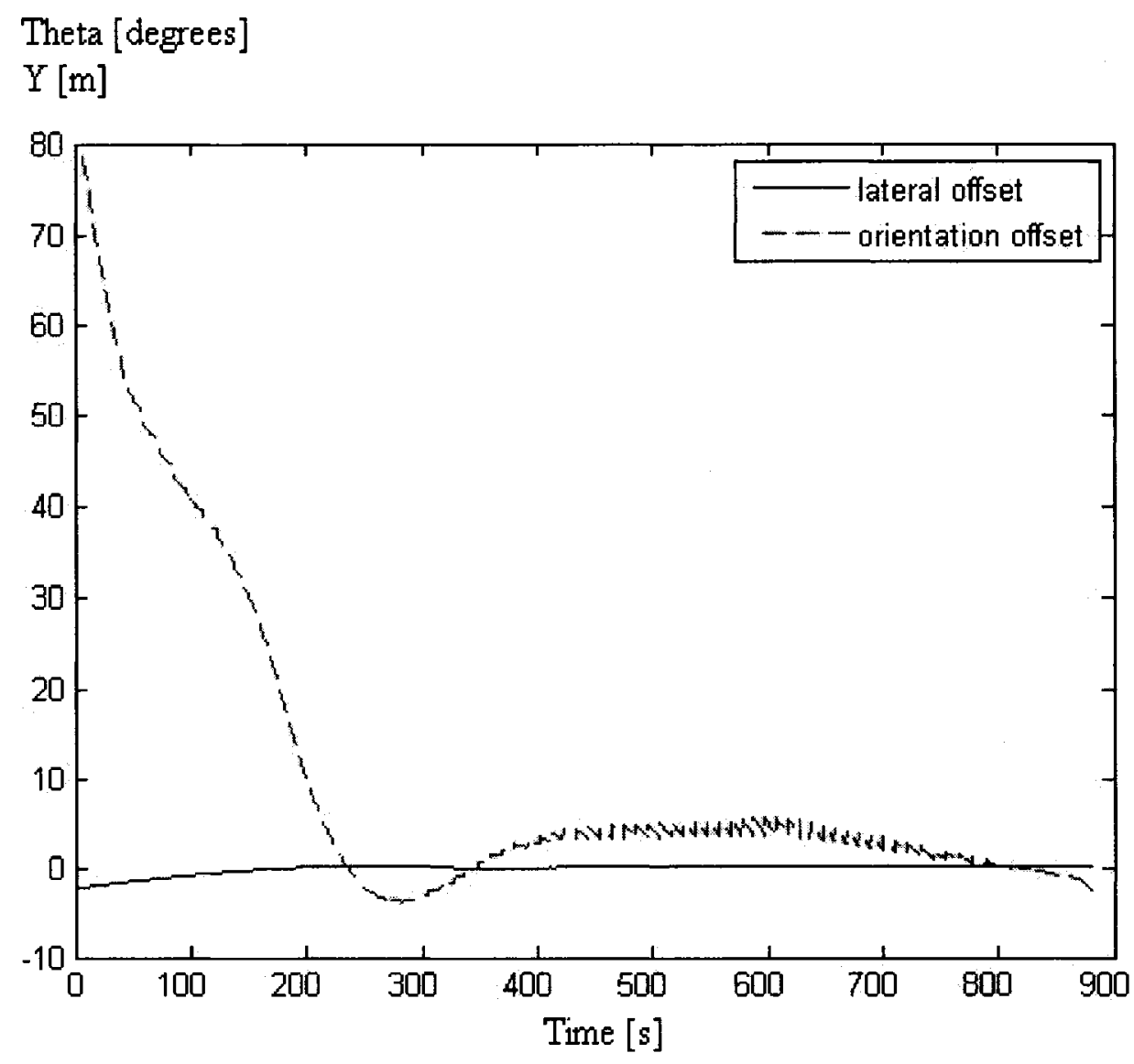

Figure 7.24: Trajectory tracking and offsets for curved path with $C_{1}=20$ and $C_{2}=10$

\subsubsection{Scanning and mapping of environment}

After moving a step with control input signal, the next step of programming is to scan the environment surrounding robot so as to map it. This map is used to detect the presence of obstacle in the way of robot. As discussed in chapter 4 , scanning is done by laser range scanner. The range and azimuth angle of the obstacle points obtained consists of noise and needs to be filtered out. EKF is used to carry out filtering using equations (4.15) to (4.15). Since the simulation time is chosen to 0.1 second the linearized dynamic matrix for prediction model is given as: 


$$
\mathbf{F}_{\rho}=\left(\begin{array}{cc}
1 & \Delta t \\
0 & 1
\end{array}\right)=\left(\begin{array}{cc}
1 & 0.1 \\
0 & 1
\end{array}\right)
$$

And system noise covariance matrix is given as:

$$
\mathbf{Q}_{\rho}=\left(\begin{array}{cc}
\frac{\Delta t^{4}}{4} & \frac{\Delta t^{3}}{2} \\
\frac{\Delta t^{3}}{2} & \Delta t^{2}
\end{array}\right) \sigma^{2} q=\left(\begin{array}{cc}
0.000025 & 0.0005 \\
0.0005 & 0.01
\end{array}\right) \sigma^{2} q
$$

Where, $\sigma^{2} q$ is the range variance and can be calculated using equation (4.15). These filtered results are used to update the occupancy grid probabilities using the sets of equations as (4.2) (4.8). The problem faced during decision of whether the grid is occupied when the updated probabilities are below 0.5 because all grids were initiated using this value of probability. Hence, slight modifications are made so as to decide particular grid is occupied or empty in which, during each scan before updating probabilities using inverse sensor model algorithm shown in section 4.5, the grid in which obstacle is detected is assigned value of 1 . These grids having value 1 are used for obstacle avoidance and steering angle determination. Hence, at each step the grids detected as occupied in previous step is not processed again and this reduces processing time and memory. Those grids identified with obstacle at each step are stored for representation of grid at the end of simulation. After assigning probabilities for each sweep, the grids with probability 1 are updated using Bayesian formula. The occupied grids are further processed to identify end points of obstacle as already discussed in chapter 5. When the object is detected, the filtered range and azimuth angle can be plotted as figure below representing location of obstacle with respect to robots view. 


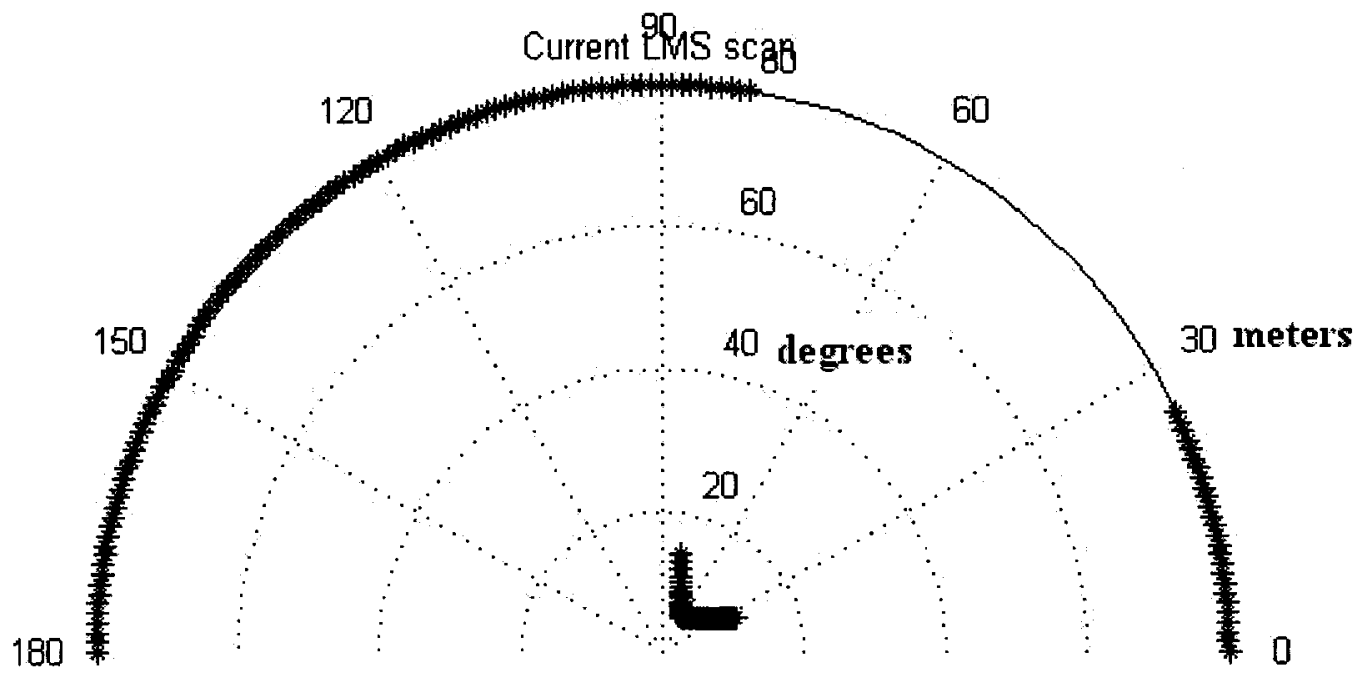

Figure 7.25: Local scan view using LMS for one obstacle.

Filtered LMS scan

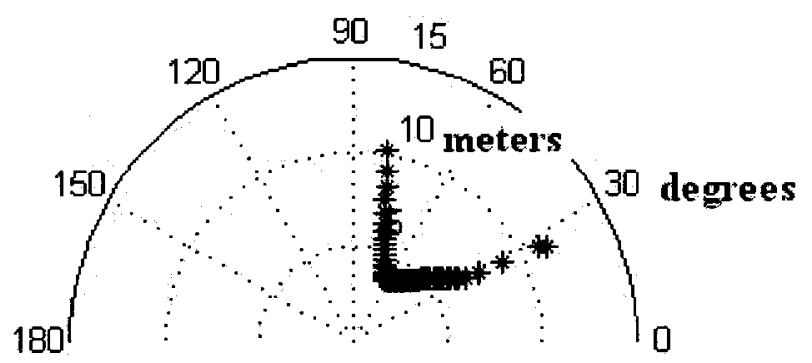

Figure 7.26: Local scan view using LMS for one obstacle. 


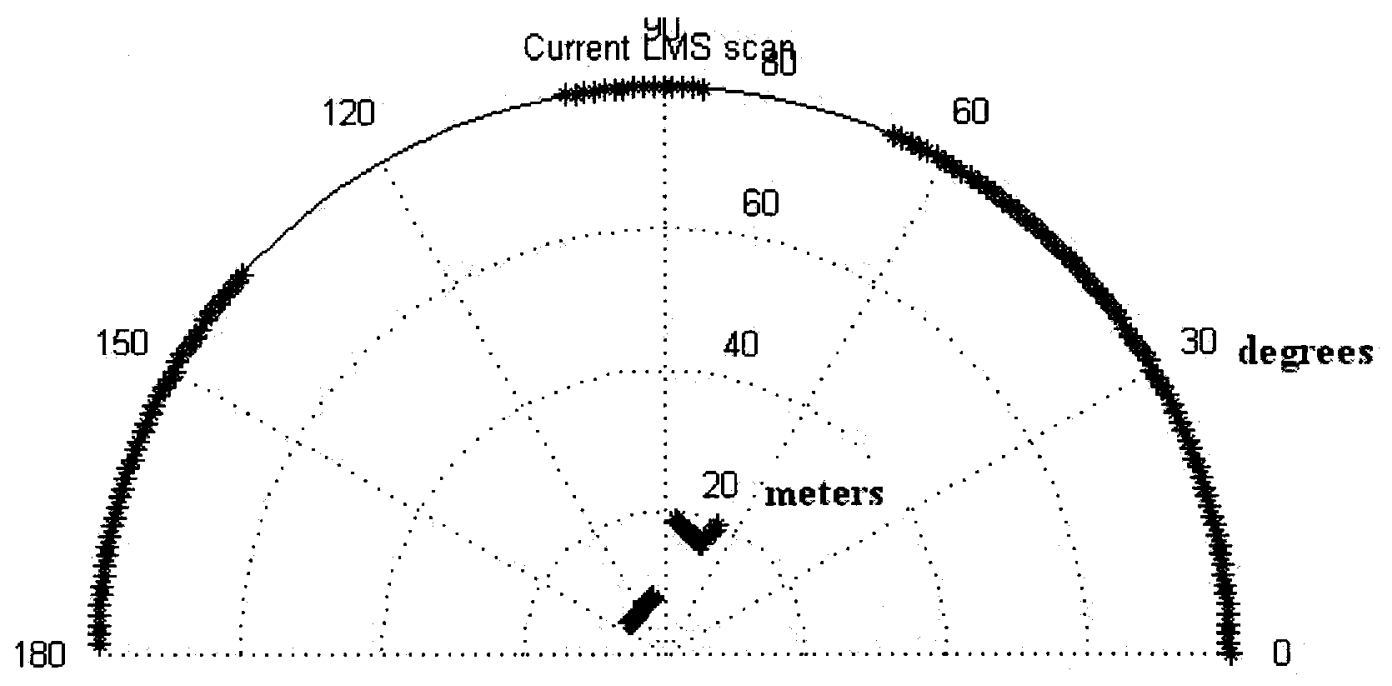

Figure 7.27: Local scan view using LMS for two obstacles.

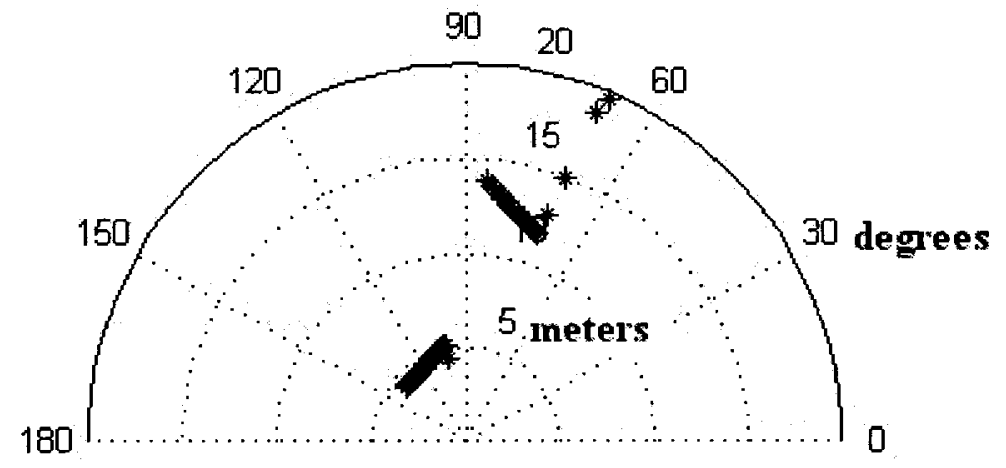

Figure 7.28: Local scan view of LMS after filtering data within maximum range.

This data is further processed to generate and update the grids probabilities. For this the grids that makes and angle of $+\mid-65$ degrees about robots longitudinal axis and those that are within 3 meters from robot are searched and only those grids are assigned probabilities using inverse sensor model. This will reduce computational time. This is as shown in figure below: 
(a)
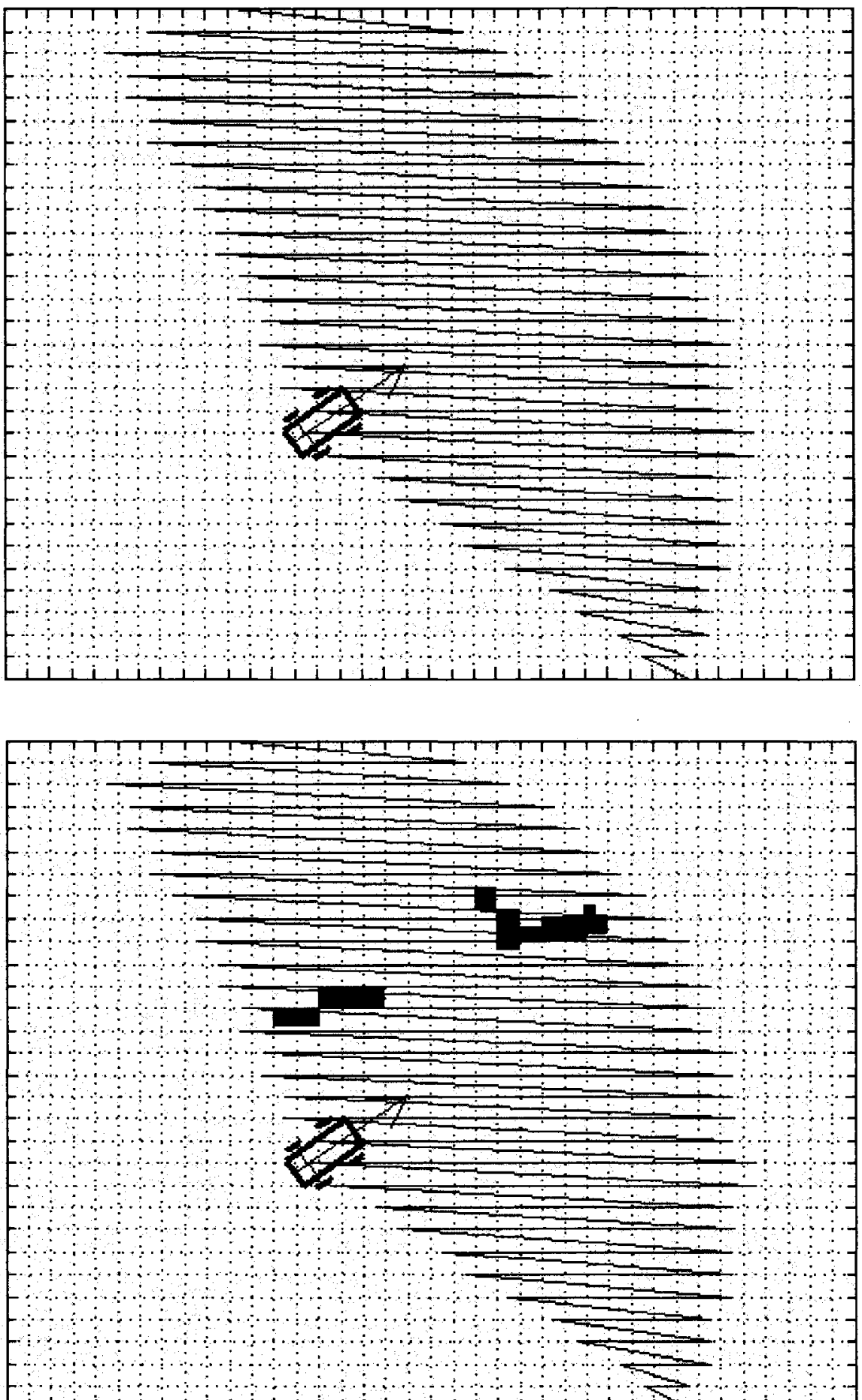

(b)

Figure 7.29: Grids accounted for single sweep of LMS (a) and occupied grids (b) 


\subsubsection{Obstacle avoidance and steering angle determination}

After generation of grid, the next step in simulation is to process these grids. Number of objects is counted first and accordingly collision avoidance algorithm is picked up depending upon single or multiple objects are detected. As shown in figure 7.29, it's the case of multiple objects. If the distance between two points is more than 4 meters then they are considered as different objects so that to keep sufficient clearance if robot chooses to traverse between two objects. After counting number of objects, the end points of objects are picked up. These are the grid reference points consisting obstacle end points. Using these points and considering whether the object is on or away from the trajectory and determining one, two or three detection case; obstacle end point is selected using algorithm defined in section 6.2.2 and finally target point using algorithm given in 6.2.3. Steering angle is calculated using algorithm discussed is diction 6.2.4. In this example shown in figure 7.29 , if the robot is away from the trajectory, target point is selected as second point of first objects if the local angle made by all obstacle end points is smallest with second point of first object; hence the direction chosen to maneuver in next step should be between two objects. Finally in case where object comes very close within the distance of 3 meters, collision is avoided using algorithm in section 6.2.6. The desired steering angle is compared with current steering angle to determine offset and is provided to PD controller to calculate required rate of change of steering or steering control signal. The results for collision avoidance for different cases are discussed below. All figures are scaled with 1 unit as 1 meter in $\mathrm{X}$ and $\mathrm{Y}$ direction. First we will see some results with one object and then with multiple objects. 
1) 1 object, 1 detection on right

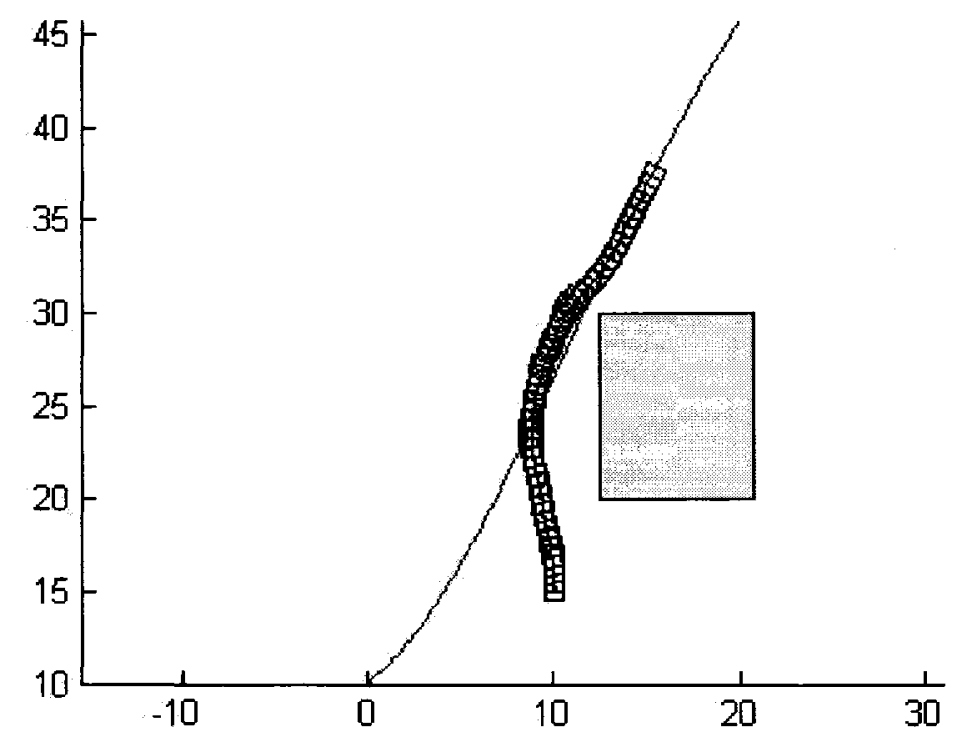

(a)

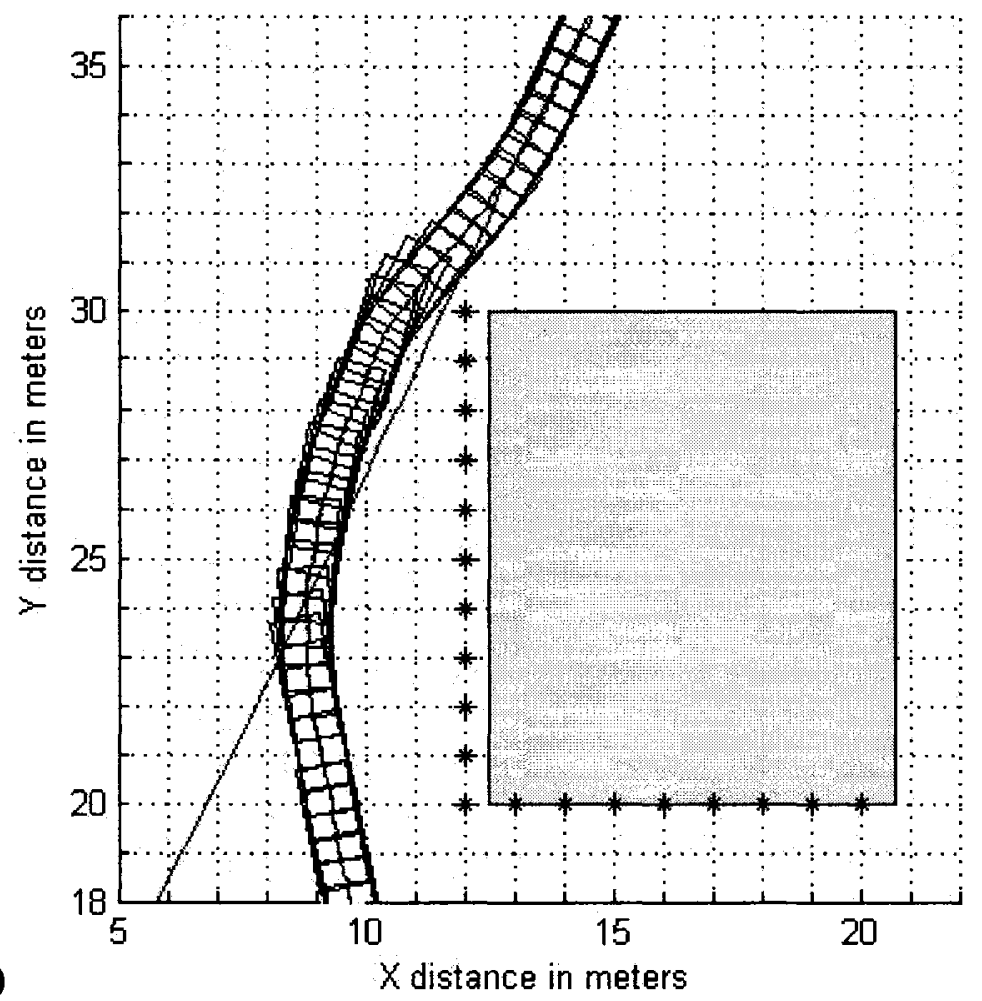

Figure 7.30: Collision avoidance with one object, one detection on right off trajectory 
Here, the grids that are found occupied marked with ' $*$ ' in figure above during a single sweep are allocated with probability 1 that is $p\left(r_{t} \mid m_{x, y}\right)=1$. Based on these grids only collision avoidance algorithm is performed. These grids are updated using Bayesian formula (4.2) as:

$$
\begin{aligned}
p\left(m_{x, y} \mid r_{1}, r_{2}, \ldots, r_{t}\right) & =\frac{p\left(r_{t} \mid m_{x, y}\right) \cdot p\left(m_{x, y} \mid r_{t-1}\right)}{p\left(r_{t} \mid r_{t-1}\right)} \\
& =\frac{1 \cdot p\left(m_{x, y} \mid r_{t-1}\right)}{p\left(r_{t} \mid r_{t-1}\right)}
\end{aligned}
$$

$p\left(r_{t} \mid r_{t-1}\right)=\sum_{i=0}^{n} P_{(t-1) x, y^{i}}^{.1}$ is the sum of product of probabilities of grids having probability 1 with previous probability of corresponding grid.

where, $\mathrm{i}=$ number of grid with probability 1 during one sweep.

If the sum is equal to 1 and the previous probability of particular grid at any previous time step is 0.5 then the updated probability is equal to $\frac{1 \times 0.5}{1}=0.5$. This process is repeated for each grid with probability 1 for each time step.

For the case as shown in figure (7.30), with a single object found in front and right zones of robot. The algorithm regarding selection of goal point and obstacle end point is shown in section 6.2.3 and 6.2.2 respectively. In this case, consider that if the local angle made by final goal point is 90 degrees (exactly front of robot) then the obstacle grid with 
smallest angle difference between the line connecting final goal point and robot reference point and obstacle grid itself with robot reference point will be selected end point.

\section{2) 1 object, 2 detection on left and front}

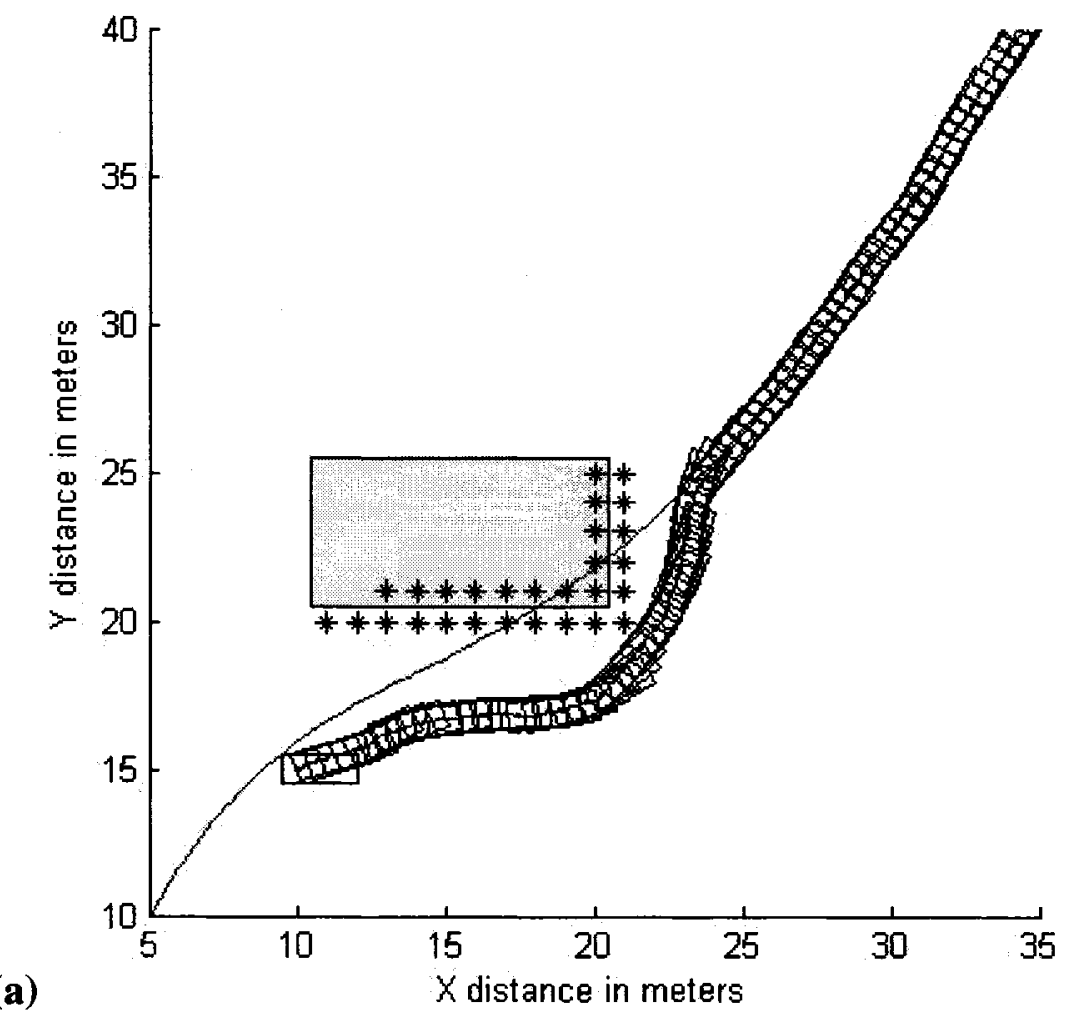


distance $\mathrm{Y}$ in meters

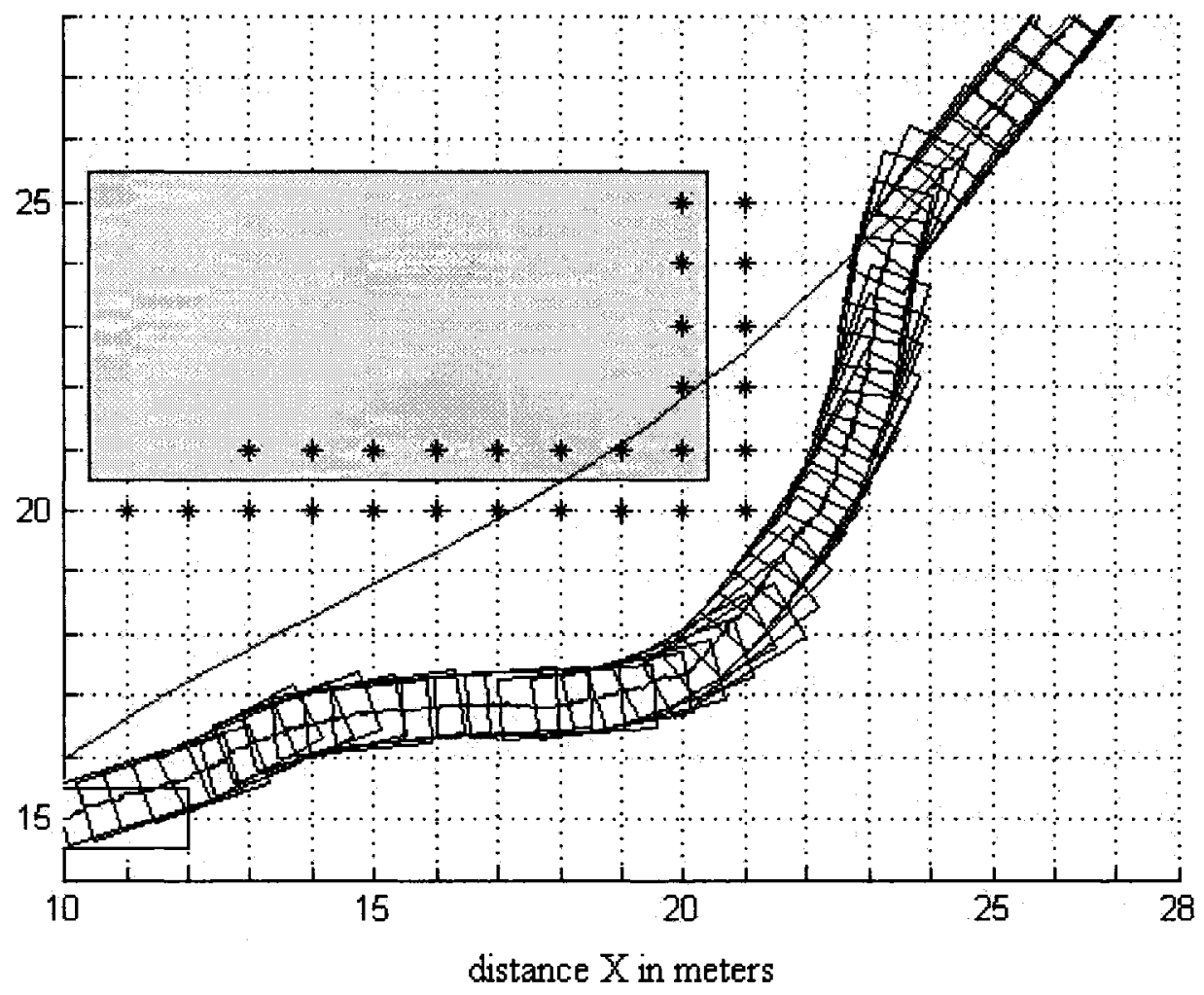

(b)

Figure 7.31: Collision avoidance with one object, two detection on left and front off trajectory 
3) 1 object, 3 detection in front, left and right

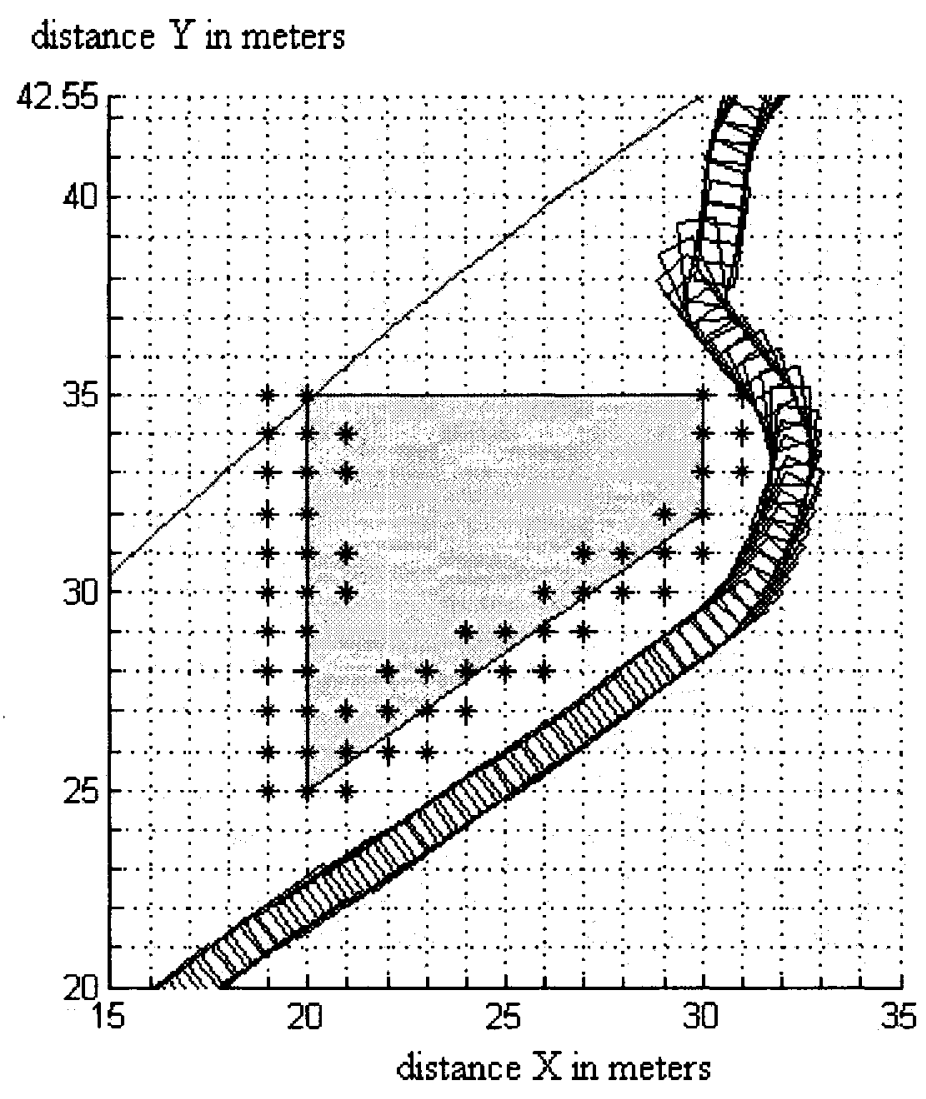

Figure 7.32: Collision avoidance with one object, all three detection off trajectory

From all these three results, it can be seen that the robot tries to keep safe distance of 3 meters from the obstacle occupied grids. The robot while moving on its path scans for obstacle and maps the same using occupancy grids as discussed previously. The detection of grids occupied with an obstacle is indicated by '*' in figure above. In first two cases, while leaving an obstacle after avoiding it, the robot attempts to come back on trajectory. During this situation there is a possibility of cutting edge of an obstacle which is avoided by implementation of presence of close objects algorithm as discussed in section 6.2.6. Since the resolution of grid is 1 meter, a single object detected in only one grid is also considered for collision avoidance. In third case, after leaving object, program searches 
for the point on trajectory making minimum distance with robot current location and determines steering angle in that direction but as soon as robot reaches 3 meters from trajectory the selection of target point on the trajectory will be some point added to point making minimum distance, otherwise, robot will approach trajectory in perpendicular direction and hence it will not converge as shown in figure 7.32.

4) 1 object, robot on trajectory with object away from 2 meters either side of robot.

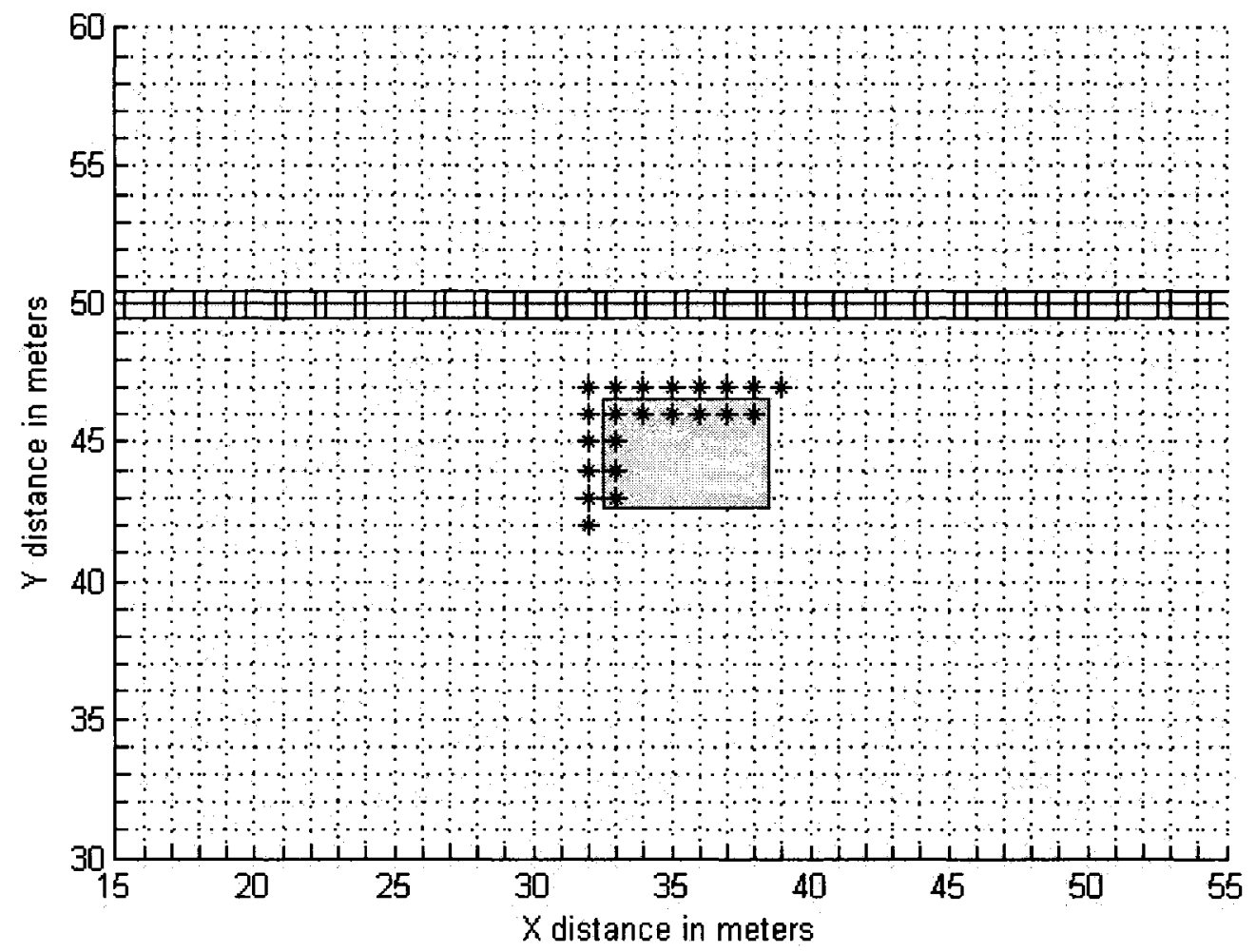

Figure 7.33: Presence of object from 2 meters away from trajectory. 
5) 1 object, robot on trajectory with object on trajectory.

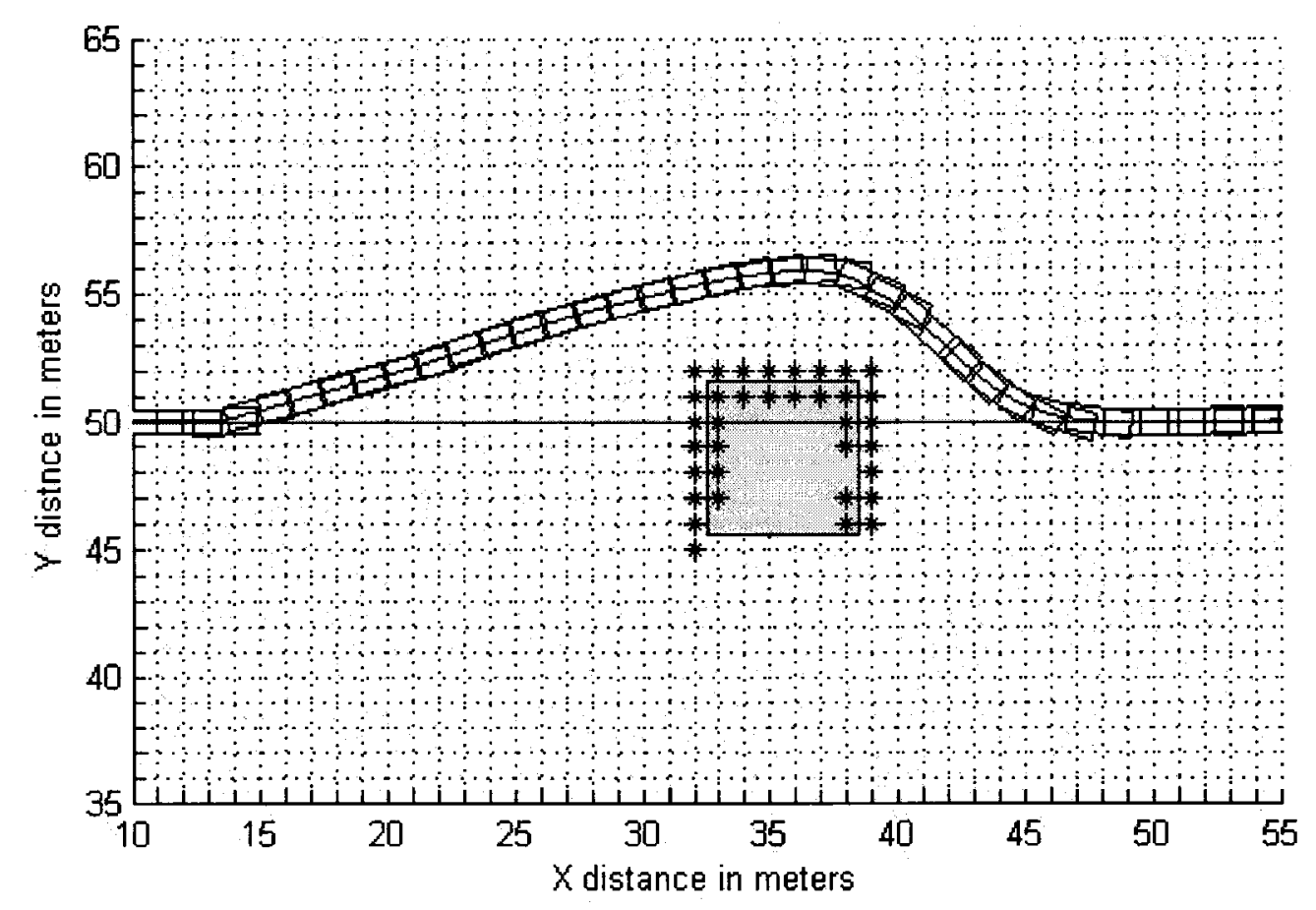

Figure 7.34: Presence of object on the trajectory

In these two cases, when robot is moving on trajectory, the program keeps on checking the presence of obstacle within 2 meters distance on either side of trajectory. If the object is away from trajectory by 2 meters on its either side, it is no more considered as in obstacle and robot will continue its motion along the trajectory. In fifth case, it can be seen that object is on trajectory; hence as per program, it will check for the presence of point on trajectory at 18-22 away from robots reference point as decided by section 6.2.2. If the goal point is right behind the object then as per section 6.2.2 the selected target point will be a point somewhere close to second point of an object with some safe distance. Selection of target point is discussed in section 6.2.3. This scenario is also seen in figure 7.14. In all above cases it can be seen that collision avoidance algorithm tries to keep safe distance of 3 meters from the obstacle. 
6) Obstacle detection from 20 meters and 15 meters.

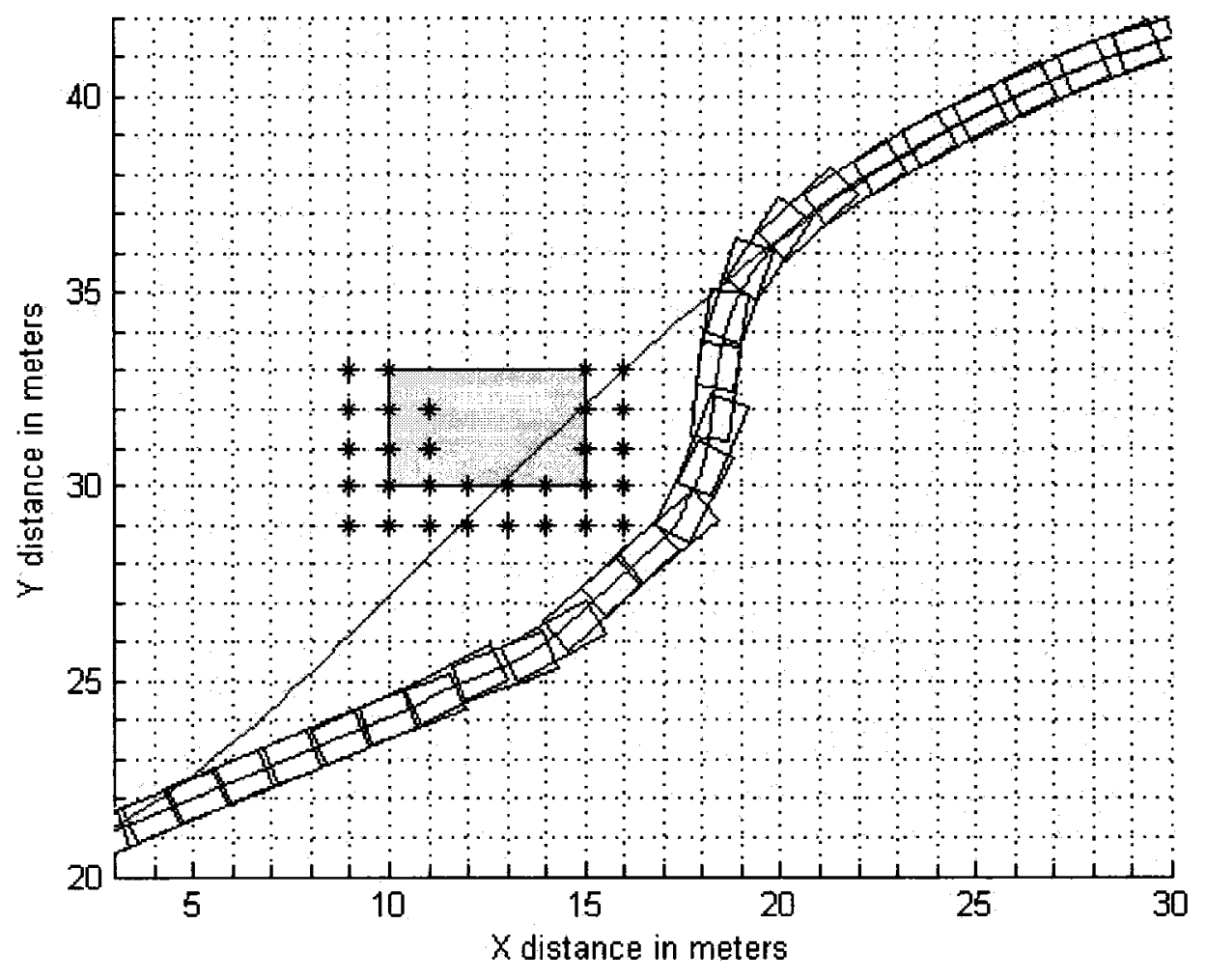

Figure 7.35: Obstacle detection from 20 meters 
(b)

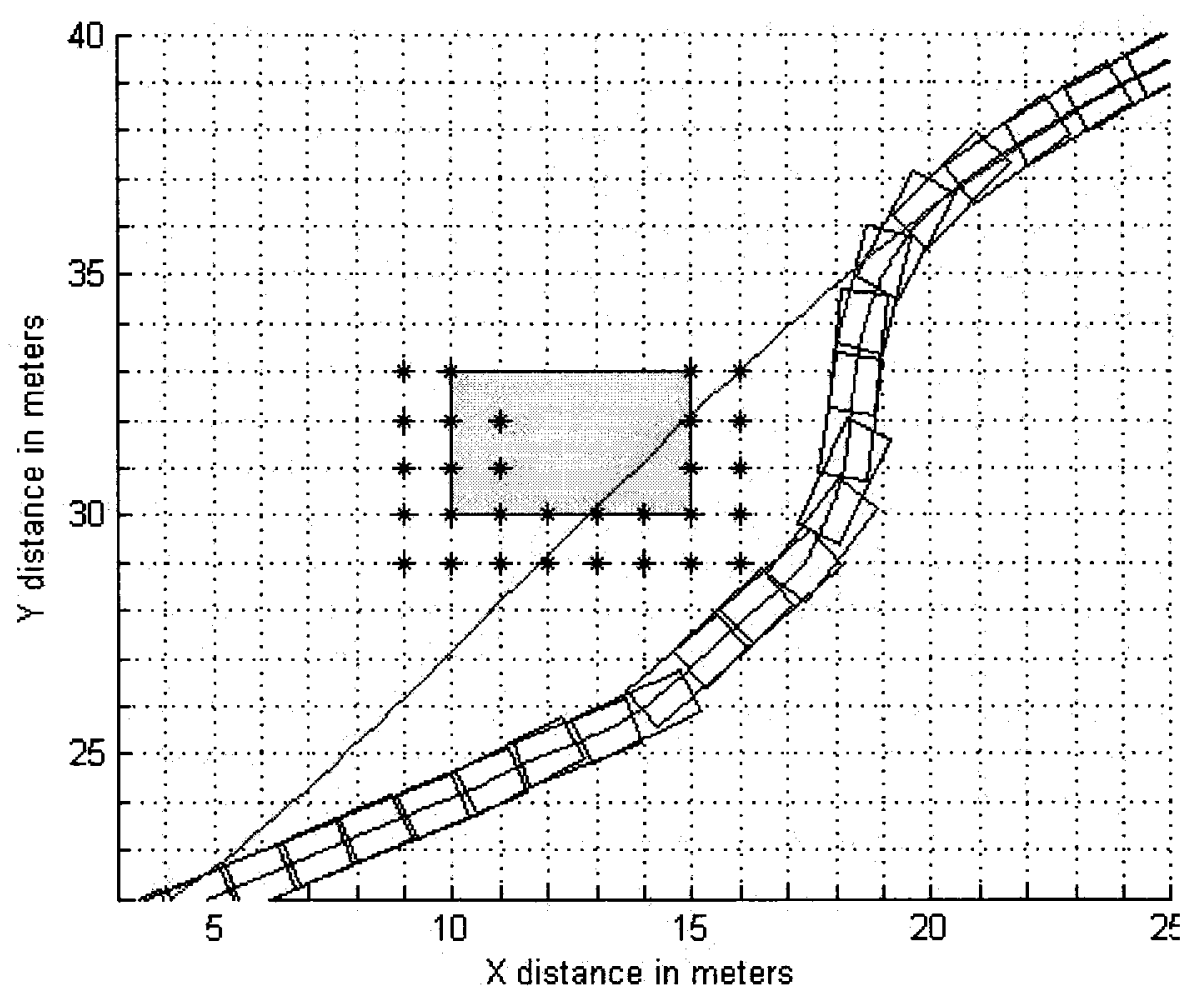

Figure 7.36: Obstacle detection from 15 meters.

The range of laser range scanner is kept to 20 meters for all previous cases. The comparison for detection of object from 20 meters and 15 meters is as shown in figure 7.35 and 7.36. In these cases, the robot is on the trajectory and as soon as it detects the obstacle on trajectory, it tries to maintain safe distance of 3 meters from the selected end point. This selected end point will keep on changing as robot progresses and received new points in next scan. From above comparison we note that when detection range is 20 meters robot starts moving away to avoid obstacle earlier than later case and as soon as robot comes near by 3 meters, the algorithm discussed in section 6.2 .6 comes into play. 
7) Two objects case

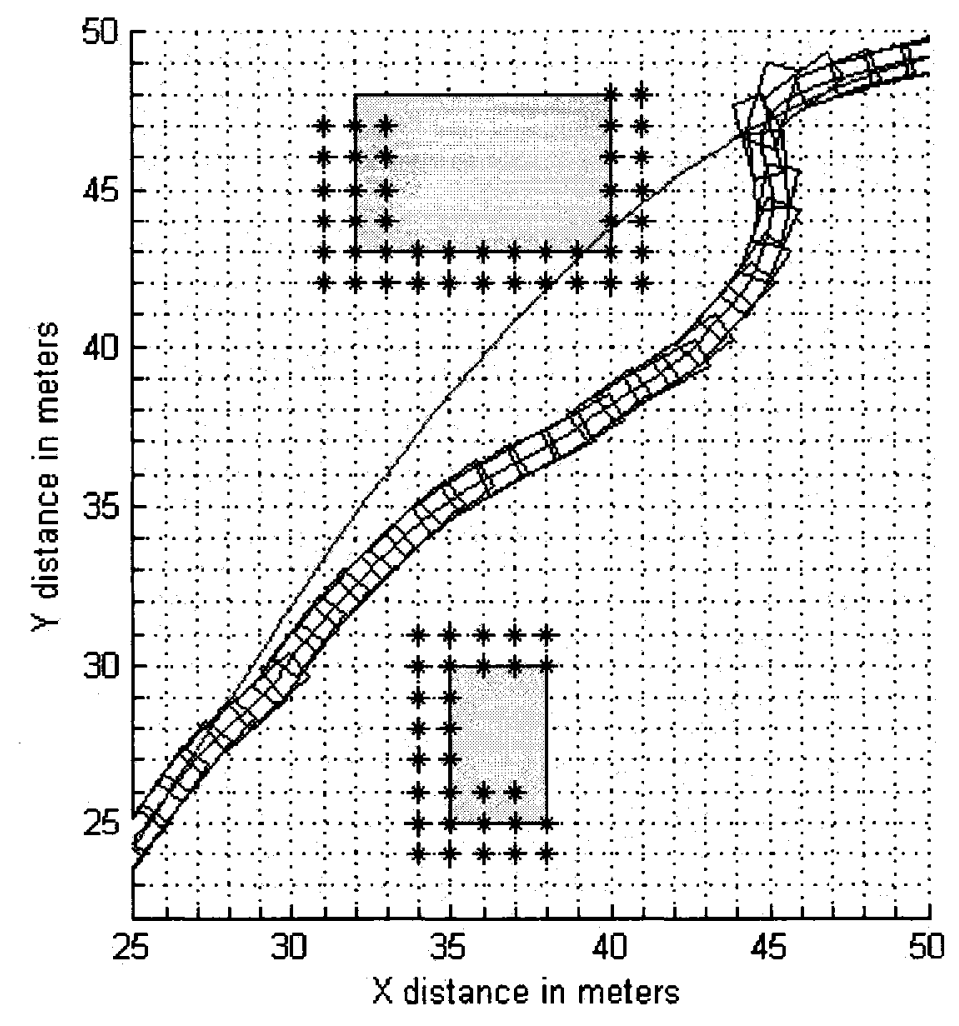

Figure 7.37: Two obstacles detection with robot moving on trajectory. 


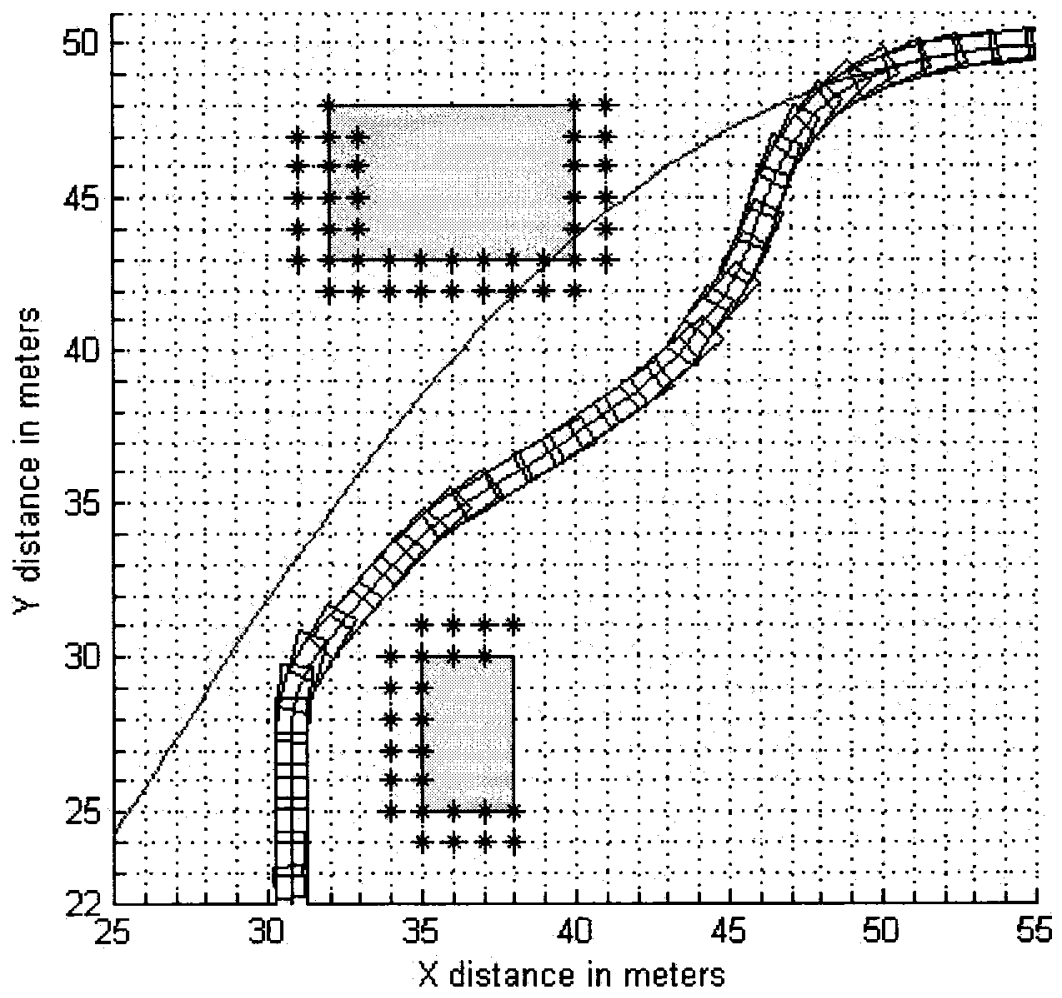

Figure 7.38: Two obstacles detection with robot away from trajectory. 
8) Three objects case

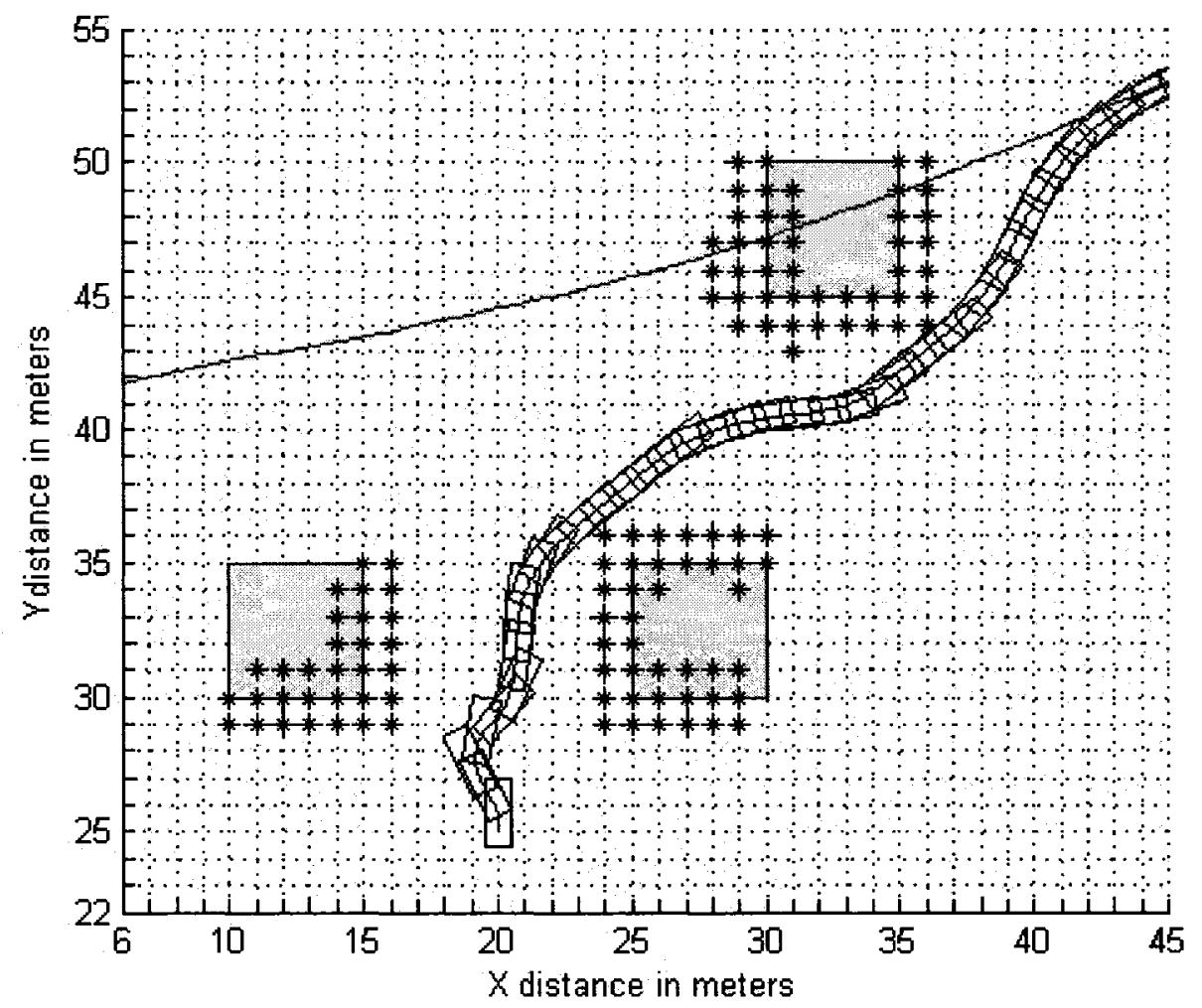

Figure 7.39: Three obstacles detection with robot away from trajectory. 
9) Five objects case

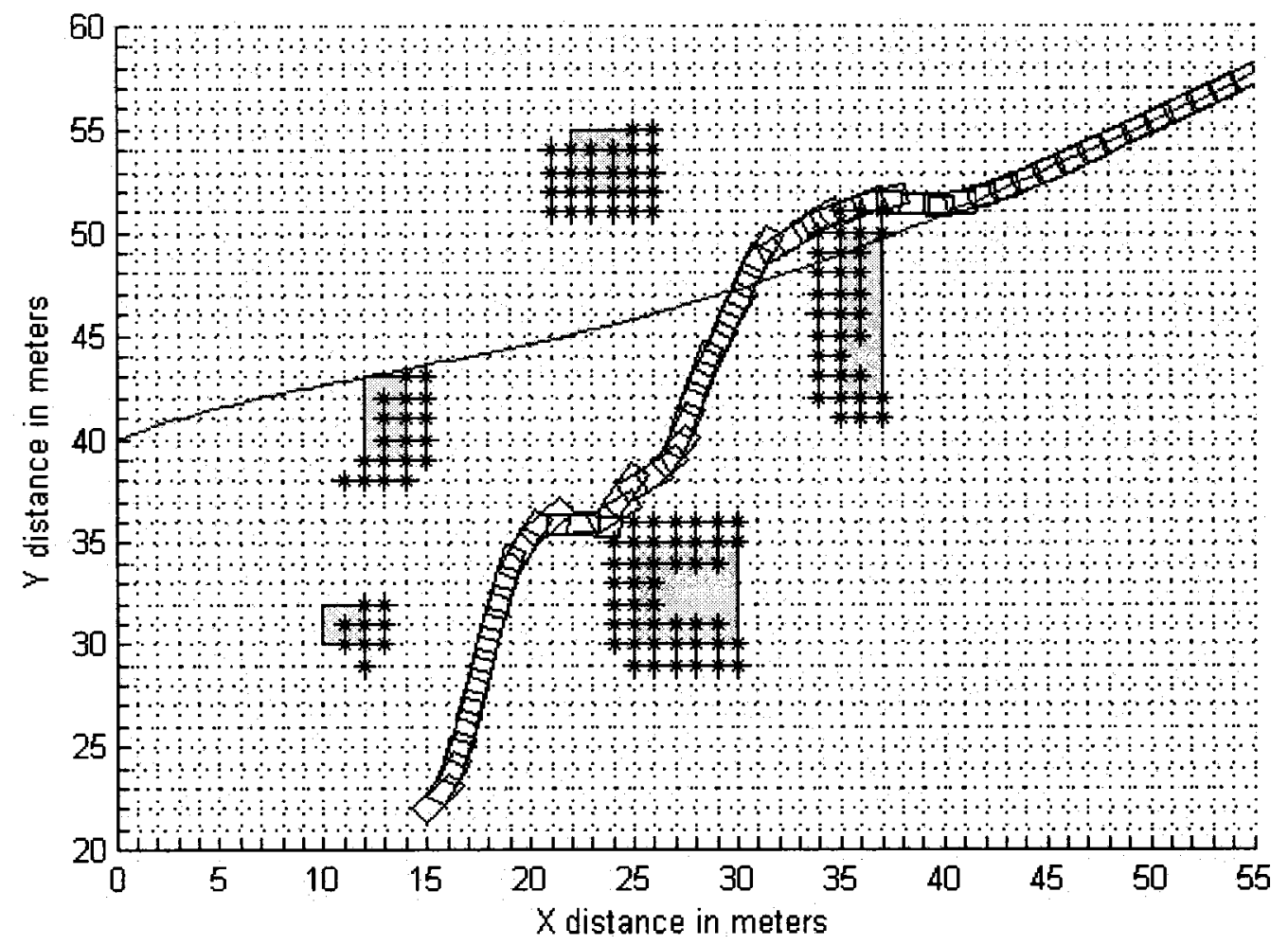

Figure 7.40: Three obstacles detection with robot away from trajectory. 
10) Robot through gate

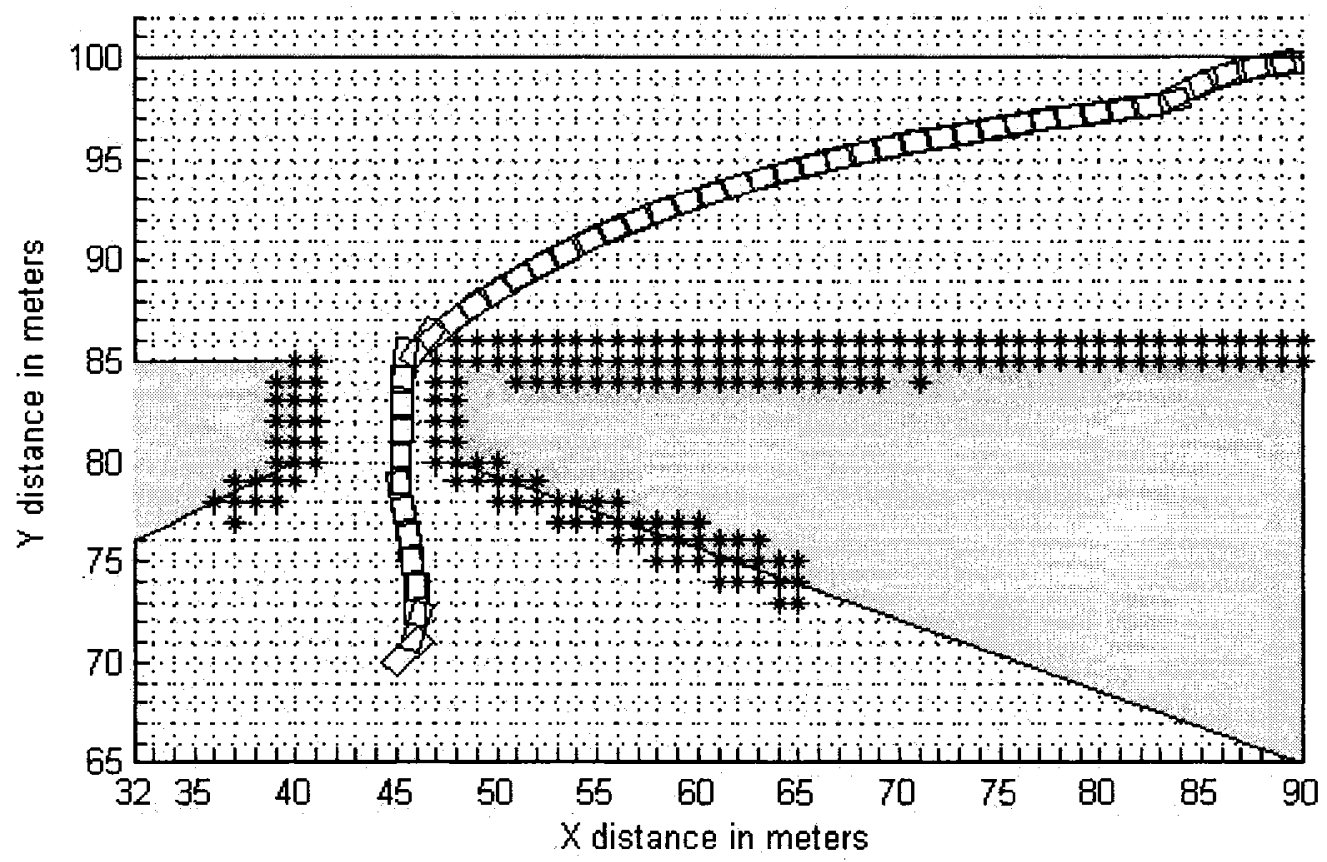

Figure 7.41: Gate with robot away from trajectory.

From this figure we can see that when the robot is away from trajectory and if the distance between two objects is greater or equal to 4 meters then it will considered as two different objects. The goal point should be the point on trajectory making minimum distance. Here, the distance after taking into consideration grids occupied is 5 meters. The algorithm shown in section 6.2.1 (second case) chooses the direction of direction of steering enabling robot to pass through middle of two objects. After coming out of gate it still detects obstacle on its right side and comes back to trajectory. 


\section{1) Robot through tunnel}

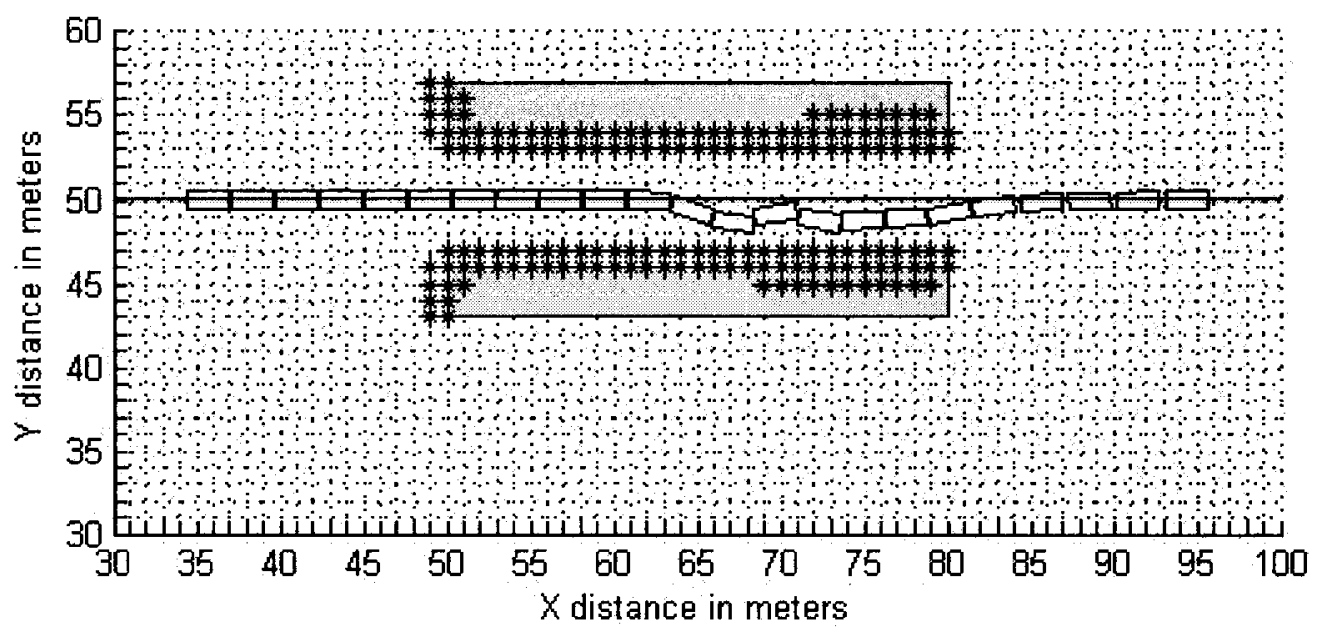

Figure 7.42: Tunnel with robot on trajectory.

From above figure it can be seen that in case of tunnel having its wall 5 meters apart after considering occupancy grids, robot is able to maintain to stay closer to designed trajectory and maintaining lateral distance from tunnel walls. As mentioned earlier, if the walls are closer than for meters than robot will consider as a single object. 
12) U shape obstacle- local minima problem

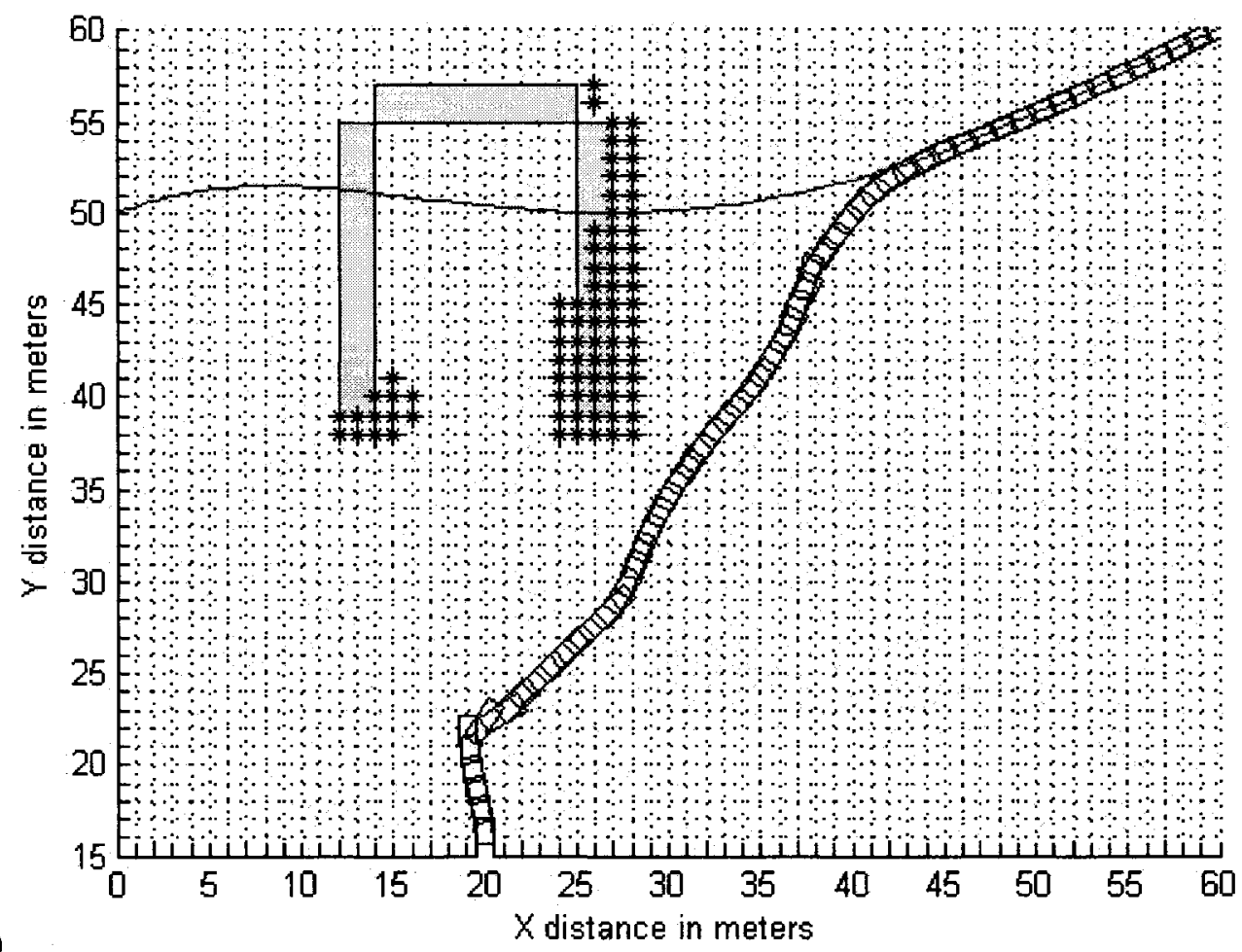

(a)

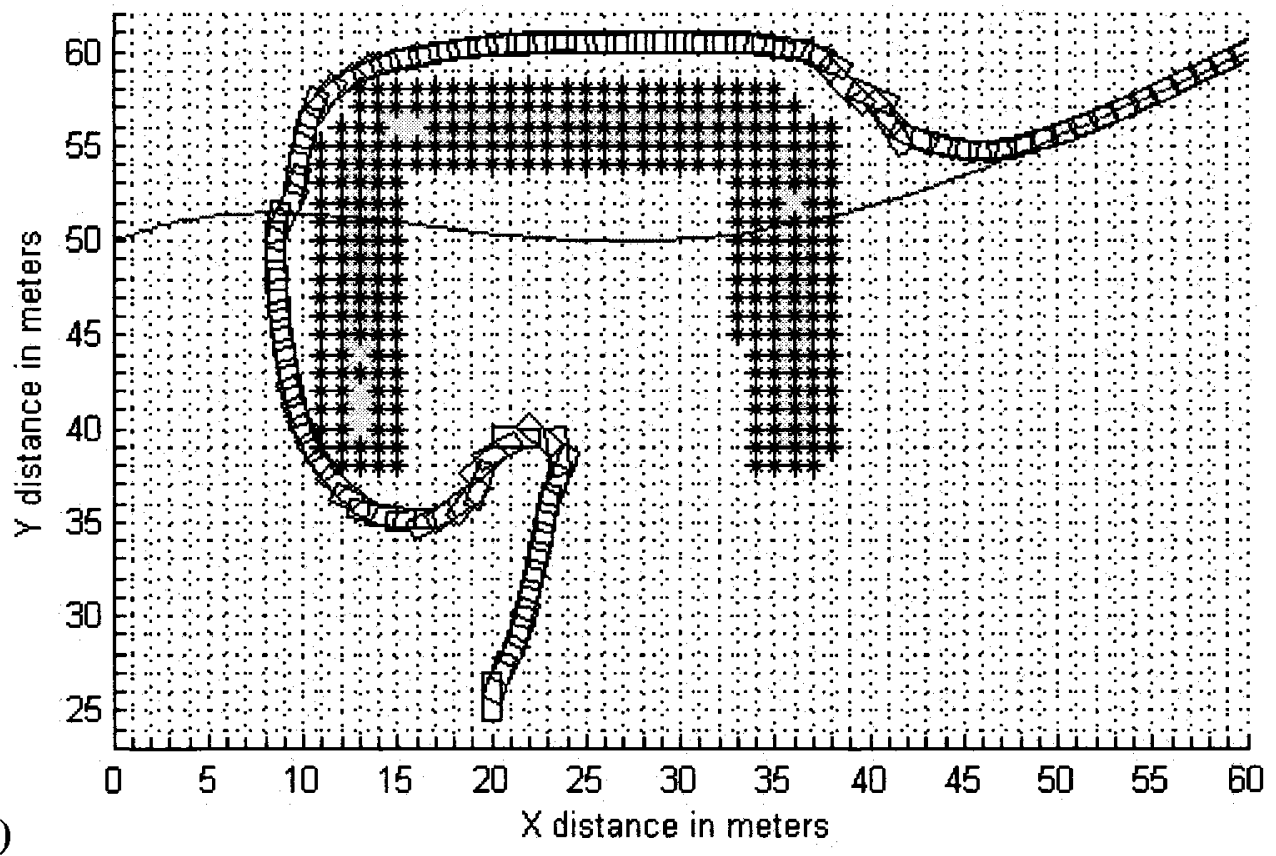

Figure 7.43: U shape obstacle- local minima problem 
As shown in figure 7.43 , for local minima problem, the algorithm tries to avoid trapping into the ' $U$ ' shaped obstacle. In figure $7.43 \mathrm{a}$, when the distance between two legs is less, the robot will not enter into the concavity of an obstacle. In figure $7.43 \mathrm{~b}$, when the distance between two legs is more then the robot first approaches near to concavity considering it as two different obstacle since from far away distance it would only see two legs of an obstacle. As soon as it moves closer to obstacle, it has full perception and obstacle and algorithm selects to steer left so as to come out of concavity, avoiding the obstacle and then finally coming back on the trajectory. Still the performance can be improved if the robot is capable of moving in reverse with backward obstacle detection. But in that case the steering angle reverses its sign compared to forward motion. 


\section{Chapter 8: Conclusions and future work}

\subsection{Conclusions}

This thesis focused on designing of autonomous mobile robot that follows dynamics and kinematics of car hence in this work the term car-like mobile robot is used for autonomous mobile robot. Being autonomous, it should be capable of executing tasks such as navigation includes localization in an unknown environment, perception and mapping the surrounding environment considering uncertainties, following pre-designed trajectory or trajectory designed during the course of its travel and finally obstacle avoidance using the map generated. Each task is designed and is implemented sequentially with information interchange to provide autonomy to car like mobile robot.

Localization is carried out by odometry calculations. Gaussian noise is assumed to be present in encoder readings and is filtered using extended Kalman filter, where different combinations of noise covariance matrices are tested and the results are shown. The output EKF is the states consisting of location of robot reference point in 2 dimensional plane and orientation with respect to reference $\mathrm{X}$ axis. The initial location and orientation of robot is known.

During motion workspace in which robot is performing specific task consisting is of uncertainties and unknown. To deal with uncertainties that cause presence of obstacles on the way, autonomous robot should acquire knowledge regarding it and has to take action 
on the basis of this knowledge acquired. For this occupancy grid method which is one of the successful methods for environment perception is implemented. In this the workspace is divided into square grids and each grid carry probability. During each step the laser range sensor sweeps $2 \mathrm{D}$ environment and its noisy output is filtered using extended Kalman filter. Using inverse sensor model of laser range finder, each grids are updated with new probabilities based on which the occupancy of grid with obstacle is decided. The performance of occupancy grid for perception of environment surrounding mobile robot and using it for collision avoidance is justified by results.

Obstacle avoidance algorithm is developed that uses occupancy grid based environmental representation indicating probable location of obstacle, its current location and orientation from EKF. Different cases depending on location of obstacle, pre-designed trajectory, number of obstacles in collision zone and location of final goal point is considered for development of obstacle avoidance algorithm. Depending upon situation the corresponding algorithm that determines desired steering angle is chosen by local path planner. During obstacle avoidance a care has been taken so that robot does not deviate away from its desired trajectory, hence reduces operating time and cost. The steering offsets are minimized using PD controller. The effectiveness of proposed algorithm is shown by results considering various cases.

Finally, trajectory tracking is achieved by implementing two controllers, viz. steering controller that generates control signal based on gains given by $L Q R$ principle facilitates tuning of feedback control law by trial and error method and propulsion or velocity 
controller that generates velocity control signal which is PI controller. The performances of controllers are shown.

\subsection{Future work}

The future work will encompass the improvement of performance of navigation of carlike mobile robot by introducing more practical aspects that it may come across during its implementation. New concepts can be thought and implemented using new algorithms developments.

The practical aspects considered in future work for improving navigation efficiency are:

- The localization is implemented using EKF assuming presence of Gaussian white noise, but in practical this may not be the case and the noise present may be colored. To take this into account localization will carried out using particle filters that is Monte Carlo based probabilistic estimation of states using probabilistic sensor and noise model. Moreover, the sensors used are only encoders. Usage of inertial navigation system such as gyroscopes, GPS, etc. may improve performance.

- Trajectory tracking controllers are implemented assuming slippage free motion. For improving accuracy of controller for planetary motions, the slip factor may be predicted and the same will be used for controller development. Also, the work surface is assumed to be flat and this may be taken into account for further development. 
- Mapping of environment using occupancy grid method is developed that use readings from laser range finder in 2D. Better occupancy grid can be generated using laser 3D images and cameras in front of robot. The signals from both sensors may be fused using extended Kalman filter or particle filters.

- It is assumed that obstacles are convex polygons in this work. The collision avoidance algorithm may be extended for non convex obstacle. The robot moves in only forward direction that may cause it to trap at local minima ( $U$ shaped obstacle). Reverse motion and backward obstacle avoidance may be adopted to avoid local minima problem.

- The obstacle avoidance algorithm developed may be transferred to real mobile robot with car like kinematic and dynamic constraints. The robot could be tested at application level. 


\section{References}

[1] J,-P. Laumond (Ed.), "Robot Motion Planning and Control”, Springer- Verlag London Limited 1998.

[2] N. Sarkar, X.Yun, V. Kumar, "Control of Mechanical Systems with Rolling Constrains: Application to Dynamic Control of Mobile Robots". The international Journal of Robotics, Vol. 13, pp. 55-50, 1994.

[3] Y. Zang, D. Hong, T. Chang, S. Velineky, "Dynamic Model Based Robust Tracking Control of a Differentially Steering Wheeled Mobile Robot", Proc. Of American Control conference, pp. 850-854, 1998.

[4] R. M. DeSantis, "Dynamic Modeling of Mechanical Systems subject to Holonomic and Nonholonomic Constraints", EPM/RT-94/04, ECOLE Polytechnique 1994.

[5] J-C Latombe, "Robot Motion Planning", Kulwer Academic Publishers, 1991.

[6] S. Aydin, H. Temeltas, "A Novel Approach to Smooth Trajectory Planning of a Mobile Robot”, IEEE Transactions on Robotics and Automation, $7^{\text {th }}$ International Workshop on Advanced Motion Control, 2002.

[7] R. M. Murray, S. S. Sastry, "Nonholonomic Motion Planning: Steering Using Sinusoids", IEEE Transactions on Automation Control, Vol. 38, No. 5, May 1993

[8] D. An and H. Wang, "VPH: A New Laser Radar Based Obstacle Avoidance Method for Intelligent Mobile Robots", Proceedings of the $5^{\text {th }}$ Congress on Intelligent control and Automation, June 15-19, 2004, Hangzhou, P.R.China. 
[9] J. Borenstein, "The Vector Field Histogram- Fast Obstacle Avoidance for Mobile Robots", IEEE Transactions on Robotics and Automation, Vol. 7, No. 3, June 1991.

[10] K. Kant, S. Zucker, "Planning Collision-Free Trajectories in Time- Varying Enivironments: A Two-Level Hierarchy”, IEEE Transactions on Robotics and Automation, International Conference on Robotics and Automation, 1988.

[11] G. Campion, G. Bastin, and B. D' Andrea-Novel, "Structural Properties and Classification of Kinematic and Dynamic Models of Wheeled Mobile Robots", IEEE Transactions on Robotics and Automatio, Vol. 12, No.1, February 1996.

[12] R. Grabowski and L. E. Navarra-Serment, "Heterogeneous Teams of Modular Robots for Mapping and Exploration", Autonomous Robots, Kluwer Academic Publishers. Manufactured in The Netherlands, 2000, 8, pp. 293-308.

[13] R.W. Brockett, R.S. Millmann, H.J. Sussmann, "Asymptotic Stability and Feedback Stabilization", in. Differential Geometric Control Theory, Eds. Berlin, Germany, Birkhauser, 1983 pp. 181-191.

[14] J. Zabezyk, "Some Comments on Stabilizability", Int. J. App. Math, Optim, 1989, Vol. 19. pp.1-9.

[15] C.C de Wit and O.J. Sordalen, "Exponential Stabilization of Mobile Robots with Nonholonomic Constraints", IEEE Transactions, Automation and Control, Vol 37.

[16] H. Nakamura, Y. Yamashital, H. Nishitanil, H. Yamamoto, "Discontinuous control of nonholonomic systems using non differentiable 
Lyapunov functions", SICE Annual Conference in Fukui, Fukui University, Japan. August 4-6, 2003.

[17] T. Y. Yang, "Mapping and Localization for Mobile Robots", MASc Thesis, Ottawa- Carleton Institute of Electrical and Computer Engineering, Department of System and Computer Engineering, 2004.

[18] R. G. Brown, P. Y. C. Hwang, "Introduction to Random Signals and Applied Signals and Applied Kalman Filtering", John Wiley \& Sons, Inc,1992.

[19] F. U. Rehman, H. Michalska, "Discontinuous Feedback stabilization of Wheeled Mobile Robots", Proceedings of the 1997 IEEE International Conference on Control Applications, Hartford, October 5-7, 1997.

[20] Hans-Joachim von der Hardt and D. W. R. Husson, "The Dead Reckoning Localization System of the Wheeled Mobile Robot ROMANE", Proceedings of the IEEE/ISICE/IRSJ International Conerence on Multisensor Fusion and Integration for Intelligent Systems, 1996.

[21] K S Chong and L Kleeman, "Accurate Odometry and Error Modelling for a Mobile Robot", IEEE International Conference on Robotics and Automation, Albuquerque USA, April 1997, pp. 2783-2788.

[22] A. Martinelli, "The Odometry Error of a Mobile Robot With a Synchronous Drive System", IEEE Transactions on Robotics and Automation, Vol. 18, No. 3, June 2002.

[23] J. Borenstein, H.R. Everett, L. Feng, "Navigating Mobile Robots, System and Techniques", A.K. Peters, Wellesley, Massachusetts. 
[24] S. Thrun, W. Burgard, and D. Fox., "Probabilistic Robots", MIT Press, Cambridge, MA, 2005.

[25] H. Choset, K. M. Lynch, S. Hutchison, G. Kantor, W. Burgard, L. E. Kavraki and S. Thrun, "Principles of Robot Motion, Theory, Algorithms, and Implementations", Massachusetts Institute of Technology, 2005.

[26] A. Aurenhammer, "Voronoi Diagrams- A Survey of a Fundamental Geometric Structure", ACM Computing Survey 23:345-405, 1991.

[27] K. Arras, N. Tomatis, B. Jensen and R. Siewart, "Multisensor on-the-fly Localization: Percision and Reliability for Applications", Robotics and Autonomous Systems , 2001, 34(2-3): 131-143.

[28] A. Elfes, "Using Occupancy Grids for Mobile robot Perception and Navigation", IEEE Computer Society, Vol. 22, Issue 6, June 1989 Page(s):46 - 57.

[29] S. Thrun, "Learning Occupancy Grid Maps with Forward Sensor Models", Proceedings IEEE/RSJ International Conference on Intelligent Robots and Systems, 2001.

[30] S. Thrun, "Learning Metric-Topological maps for indoor mobile robot navigation", Computer science department and Robotics Institute, Carnegie Mellon University, Pittsburgh, PA 15213, USA.

[31] J. A. Castellanos, J D. Tardos, “ Mobile Robot Localization and Map Building a Multisensor Fusion Approach”, Kluwer Academic publishers, 1999.

[32] W. Dong and Yi Guo, "New Trajectory Generation Methods for Nonholonomic Mobile Robots", Proceedings of the 2005 International Symposium on Collaborative Technologies and Systems, 15-20 May 2005 Page(s):353 - 358. 
[33] O. Amidi and C. Thorpe, "Integrated Mobile robot Control", 1990, 504/ SPIE Vol. 1388 Mobile robots V

[34] T. R. Kane, D. A. Levinson, "Dynamics, theory and applications", New York: McGraw-Hill, c1985.

[35] R. M. DeSantis, "Path-Tracking of Car-Like Robots with Single and Double Steering", IEEE Transactions on Vehicular Technology, Vol. 44, No. 2, May 1995.

[36] A. Fujimori, P N. Nkiforuk, and Madan M. Gupta, "Adaptive Navigation of Mobile Robots with Obstacle Avoidance", IEEE Transactions on Robotics and Automation, Vol. 13, No. 4, August 1997.

[37] D.E. Koditschek and E. Rimon, "Robot Navigation Functions on Manifolds with Boundry", Advances in Applied Mathematics, 1990, 11:412-442.

\section{Other useful references:}

[38] J. Z. Sasiadek and P. Hartana, “Adaptive Fuzzy Logic Systems for Sensor Fusion in Dead-Reckoning Mobile Robot Navigation", $15^{\text {th }}$ Triennial World Congress, Barcelona, Spain, IFAC, 2002.

[39] A. Diosi, G. Taylor and L. Kleeman, "Interactive SLAM Using Laser and Advanced Sonar", Proceedings of the IEEE International Conference on Robotics and Automation, Barcelona, Spain, April 2005.

[40] DeSantis, R.M., "Path-Tracking for Car-Like and Tractor-Trailer-Like Robots", Ecole Polytechnique de Montreal, October 28, 1993.

[41] H. Hu, M. Brady, P. Probert, "Trajectory Planning and Optimal Tracking for an Industrial Mobile Robot”, 152/ SPIE Vol. 2058 Mobile Robots VIII, 1993 
[42] C. Samson, "Control of Chained Systems Application to Path Following and Time-Varying Point-Stabilization of Mobile Robots", IEEE Transactions on Automatic Control, Vol. 40, No. 1, January 1995.

[43] R. Grabowski, P. Khosla and H. Choset, "Autonomous Exploration Via Regions of Interest", Proceedings of the 2003 IEEE/RSJ, International Conference on Intelligent Robots and Systems, Les Vegas, Nevada, October 2003.

[44] K. Dutton, S. Thompson, B. Barraclough, "The Art of Control Engineering", Addison-Wesley Longman, 1997.

[45] R. Siegwart, I R. Noirbakhsh, "Introduction to Autonomous Mobile Robots", Massachusetts Institute of Technology, 2004.

[46] D. Kortenkamp, R. Peter Bonasso, and R. Murphy, "Artificial Intelligence and Mobile Robots", AAAI Press, 1998.

[47] K. Gupta, A. P. D. Pobil, "Practical Motion Planning in Robotics, Current Approaches and Future Directions”, John Wiley \& Sons Ltd., 1998.

[48] U. Nehmzow, "Mobile Robotics: A Practical Introduction", Springer-Verlog London Limited, 2000.

[49] J. T. Schwartz, M. Sharir, J. Hopcroft, "Planning, Geometry, and Complexity", Ablex Publication corporation, 1987.

[50] P. Dorato, C. Abdallah, Vito Cerone, "Linear-Quadratic Control, An Introduction”, Prentice-Hall, Inc., 1995.

[51] H. K. Khalil, "Nonlinear Systems", Prentice-Hall Inc., 2002, 1996.

[52] R. Marino, P. Tomei, "Nonlinear Control Design", Prentice Hall International (UK) Limited, 1995. 
[53] W. E. Dixon, D. M. Dawson, E. Zergeroglu and A. Behal, "Nonlinear Control of Wheeled Mobile Robots”, Springer-Verlag London Limited, 2001.

[54] Z. Li, J. F. Canny, "Nonholonomic Motion Planning", Kluwer Academic Publishers, 1993.

[55] M. Xie, "Fundamentals of Robotics, Linking Perception to Action", World Scientific Publishing Co. Pte. Ltd., 2003.

[56] G. Dudek, M. Jenkin, "Computational Principles of Mobile Robotics”, Cambridge University Press, 2000.

[57] Yi Lu, "Modeling and Control System Design of the LHD Vehicle", MASc thesis, Ottawa- Carleton Institute of Mechanical and Aerospace Engineering, Department of Mechanical and Aerospace Engineering, 2003.

D. N. Green, "Guidance of an Autonomous Planetary Rover", M.Engg, Ottawa-Carleton Institute of Mechanical and Aerospace Engineering, Department of Mechanical and Aerospace Engineering, 1994. 


\section{Appendices}

\section{1) Algorithm for the flow of simulation}

Step 1:Initialization of variables, trajectory and objects

Step 2:Dynamics and kinematics of robot

Step 3: Localization of robot using EKF

Step 4:Scanning 2D environment using Laser range scanner and filtering the same using EKF

Step 5:Checking for collision. If collision is present go to step 6 else jump to step 9

Step 6: Generation of Occupancy grid using LMS readings from step 5.

Step 7:Determine necessary steering angle for collision avoidance depending on number of objects and location of trajectory and final goal point with respect to robot reference point.

Step 8:Determine PD controller gain for control of steering angle.

Step 9:If collision not present then determine orientation, lateral distance and steering angle offsets.

Step 10: Determine steering control input using LQR gains.

Step 11: Determine propulsion control input for moving robot with given speed.

Step 12: Check if destination point is reached? If yes then terminate the program or continue to move. 


\section{2) Simulation program code in MATLAB.}

$\% \% \%$ Main program in MATLAB that calls different special purpose functions $\% \% \%$ to carry out specific task

clear all;

clc;

$\% \% \%$ Initialization of variables, trajectory and objects

$\mathrm{P}=$ reshape(50*eye(3),9,1);

$\mathrm{Vu}=1$;

$\mathrm{l}=1.5$;

$\mathrm{fp}=1$;

fs $=-1$

delta $=0$;

Delta $=0$

$\mathrm{X} 0=[15 ; 22 ; 45] ; \quad \% \% \%$ Initial location of Robot

$\mathrm{q0}=[0 ; 40 ; 20 ; 0] ; \quad \% \% \%$ Initial trajectory configuration

qf $=[60 ; 60 ; 25 ; 0] ; \quad \% \% \%$ final trajectory configuration

$\mathrm{t} 0=0$;

$\mathrm{tf}=10$ $\% \% \%$ Initial time

$\% \% \%$ Final time

$\mathrm{ts}=0.1$;

$\% \%$ Sampling time

Tos $=0 ;$ Los $=0$;

$\mathrm{Dd}=0 ; \mathrm{nu}=0 ;$ count $=1$;

[qd,Nt]=gentraj(q0,qf,t0,tf,ts);

$\operatorname{xd}=\mathrm{qd}(:, 1) ; \mathrm{yd}=\mathrm{qd}(:, 2) ;$ thetad $=\mathrm{qd}(:, 4)$;

des $=0$;

$\% \% \%$ Objects generation

objects(1).segments(1).ends=[25 35;30 35];

objects(1).segments(2).ends $=\left[\begin{array}{lll}30 & 35 ; 30 & 30\end{array}\right]$;

objects(1).segments(3).ends=[30 30;25 30];

objects(1).segments(4).ends=[25 30;25 35];

objects(2).segments(1).ends=[12 39;12 43];

objects(2).segments(2).ends=[12 43;14 43];

objects(2).segments(3).ends=[14 43;14 39];

objects(2).segments(4).ends=[14 39;12 39];

objects(3).segments $(1)$.ends $=\left[\begin{array}{lll}10 & 30 ; 10 & 32\end{array}\right]$;

objects(3).segments(2).ends $=\left[\begin{array}{lll}10 & 32 ; 12 & 32\end{array}\right]$;

objects(3).segments(3).ends=[12 32;12 30];

objects(3).segments(4).ends=[12 30;10 30];

objects(4).segments(1).ends $=[2540 ; 2542]$;

objects(4).segments(2).ends $=[2542 ; 2742]$;

objects(4).segments(3).ends=[27 42;27 40];

objects(4).segments(4).ends=[27 40;25 40]; 
objects(5).segments(1).ends $=[22$ 52;22 55];

objects(5).segments(2).ends $=[22$ 55;25 55];

objects(5).segments(3).ends $=[25$ 55;25 52];

objects(5).segments(4).ends=[25 52;22 52];

objects(6).segments(1).ends $=[3542 ; 35$ 50];

objects(6).segments(2).ends $=[35$ 50;37 50];

objects(6).segments(3).ends $=[37$ 50;37 42];

objects(6).segments(4).ends $=[3742 ; 3542]$;

$\mathrm{wx}=65 ; \mathrm{wy}=60 ; \mathrm{mxy}=0.5 ; \mathrm{m}=0 ; \mathrm{Gx}=0 ; \mathrm{Gy}=0$;

$\mathrm{C} 2 \mathrm{x}=0 ; \mathrm{C} 2 \mathrm{y}=0 ;$ Gangle $=0 ;$ Range $=0$;

[Pro]=inigrid(wx,wy, $\mathrm{mxy}) ; \quad \% \% \%$ Occupancy grid initialization

res $=1$;

max_range $=20$;

$\mathrm{X}=\mathrm{X} 0$;

equation_line=objequations(objects,res);

$\mathrm{i}=1$;

count $=1$;

for $\mathrm{i}=11372: 25000$

if $(X(1)>60 \mid X(2)>75)$

$\% \% \%$ loop with number of steps

break

end

$[\mathrm{D}, \mathrm{g} 1, \mathrm{~g} 3]=$ dmodel$(\mathrm{Vu}, \mathrm{fp}, \mathrm{fs}$, delta $) ; \% \% \%$ Dynamic model

$\mathrm{Vu}=\mathrm{D}(1) ; \mathrm{Vw}=\mathrm{D}(2) ; \operatorname{delta}=\mathrm{D}(3)$;

if delta $>45$

delta $=45$;

end

if delta $<-45$

delta=-45;

end

$[\mathrm{C}]=\mathrm{kmodel}(\mathrm{Vu}, \mathrm{Vw}, \mathrm{delta}, \mathrm{X}) ; \quad \% \% \%$ Kinematic model

$\mathrm{xp}=\mathrm{C}(1) ; \mathrm{yp}=\mathrm{C}(2) ; \mathrm{tp}=\mathrm{C}(3)$;

$\mathrm{P}=$ reshape $(\mathrm{P}, 3,3)$;

$[\mathrm{E}]=$ priori $(\mathrm{Vu}, \mathrm{Vw}, \mathrm{X}, \mathrm{P}) ; \quad \% \% \%$ Priori variables for $\mathrm{EKF}$

$\mathrm{p}=\operatorname{reshape}(\mathrm{E}(1: 9), 3,3)$;

$[\mathrm{N}]=$ ssensor(fs);

$[\mathrm{M}]=$ wsensor $(\mathrm{Vu})$;

Delta $=$ Delta $+0.1 * N$;

if Delta $>45$

Delta $=45$;

end

if Delta $<-45$

Delta=-45;

end

$A=$ ekff(xp,yp,tp,p,X,N,M,Vu,Vw,delta,Delta,fs); \%\%\% EKF for localization

$\mathrm{Xu}=\mathrm{A}(1: 3)$;

$\mathrm{P}=\mathrm{A}(4: 12)$; 


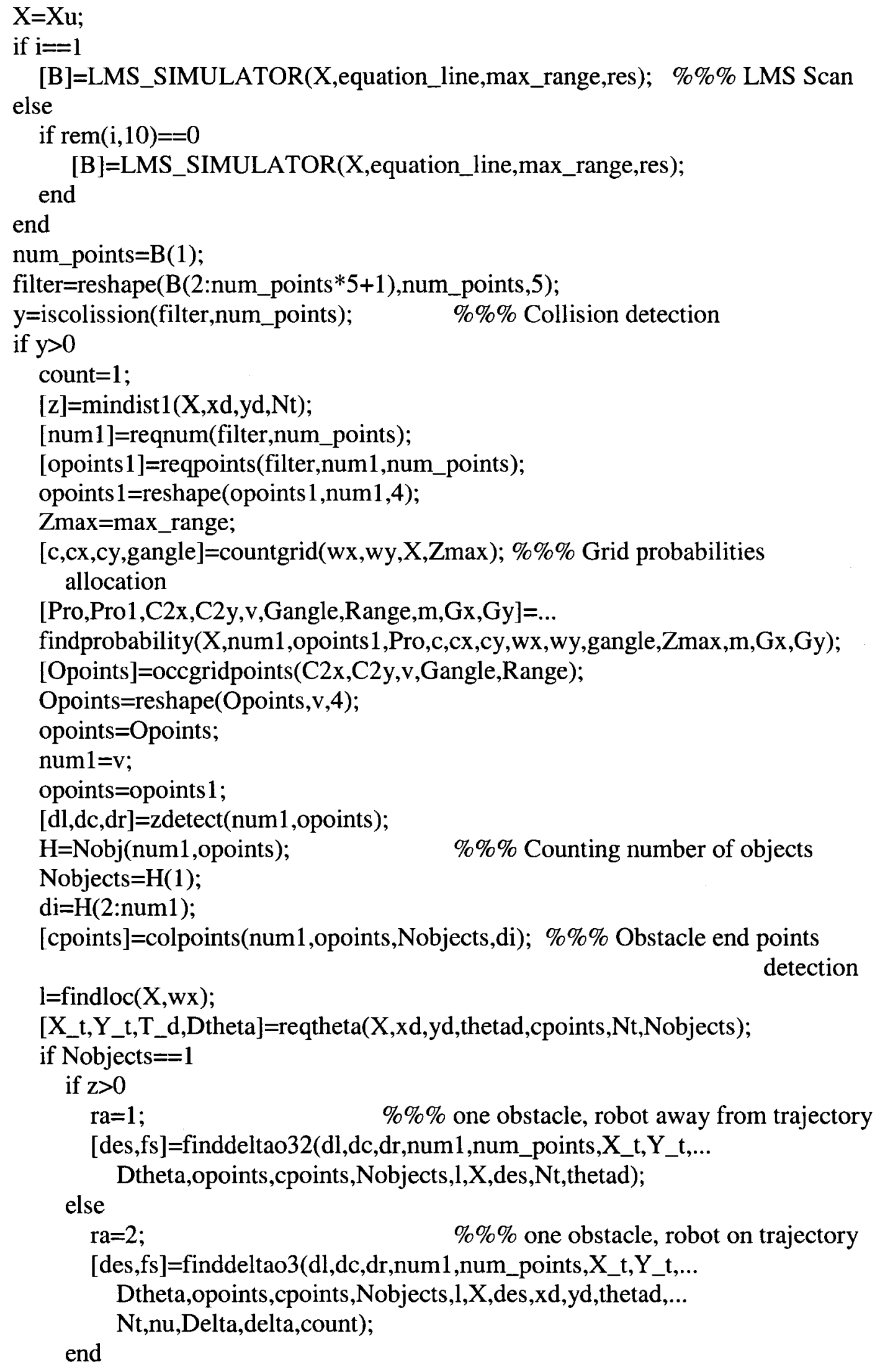




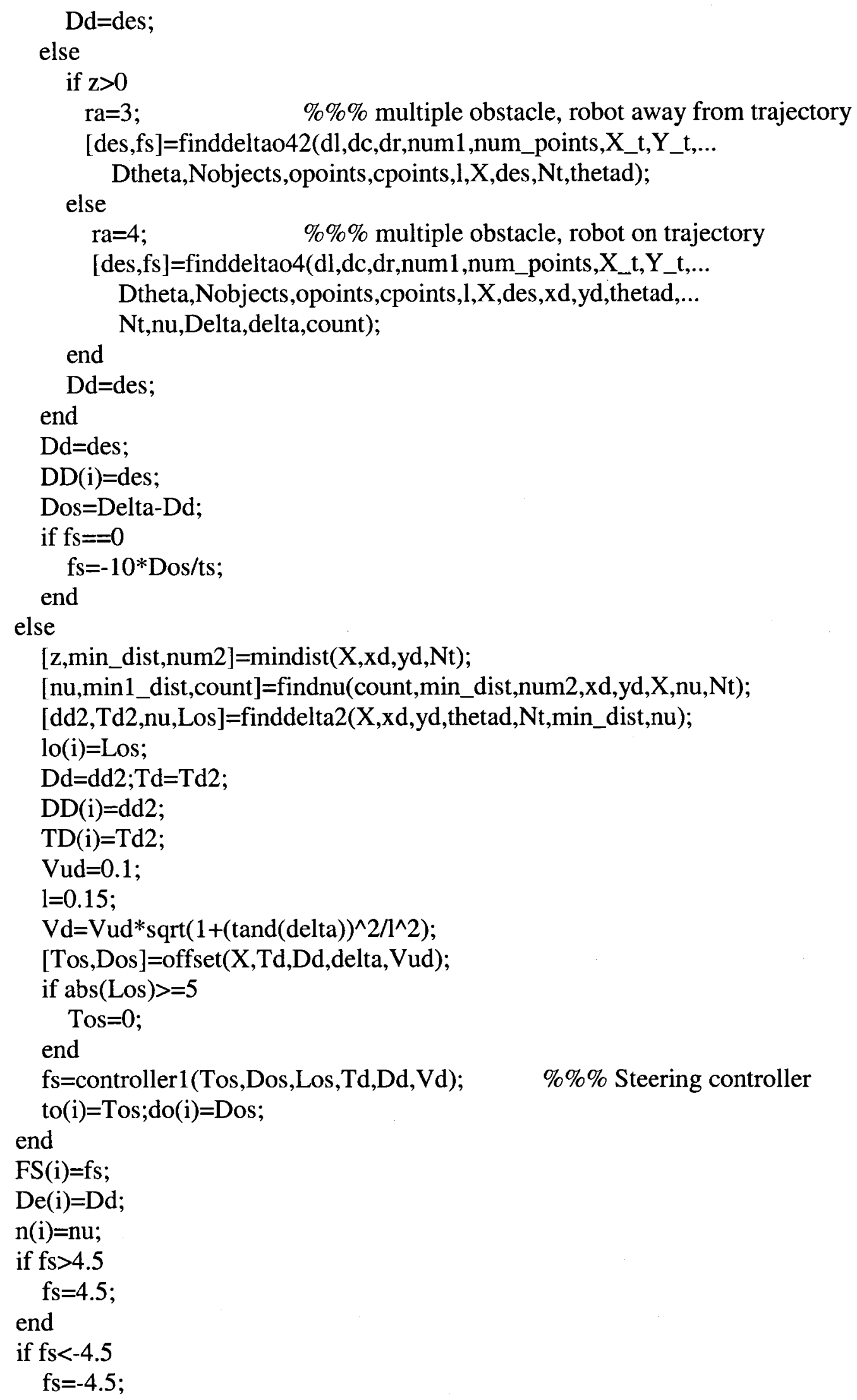




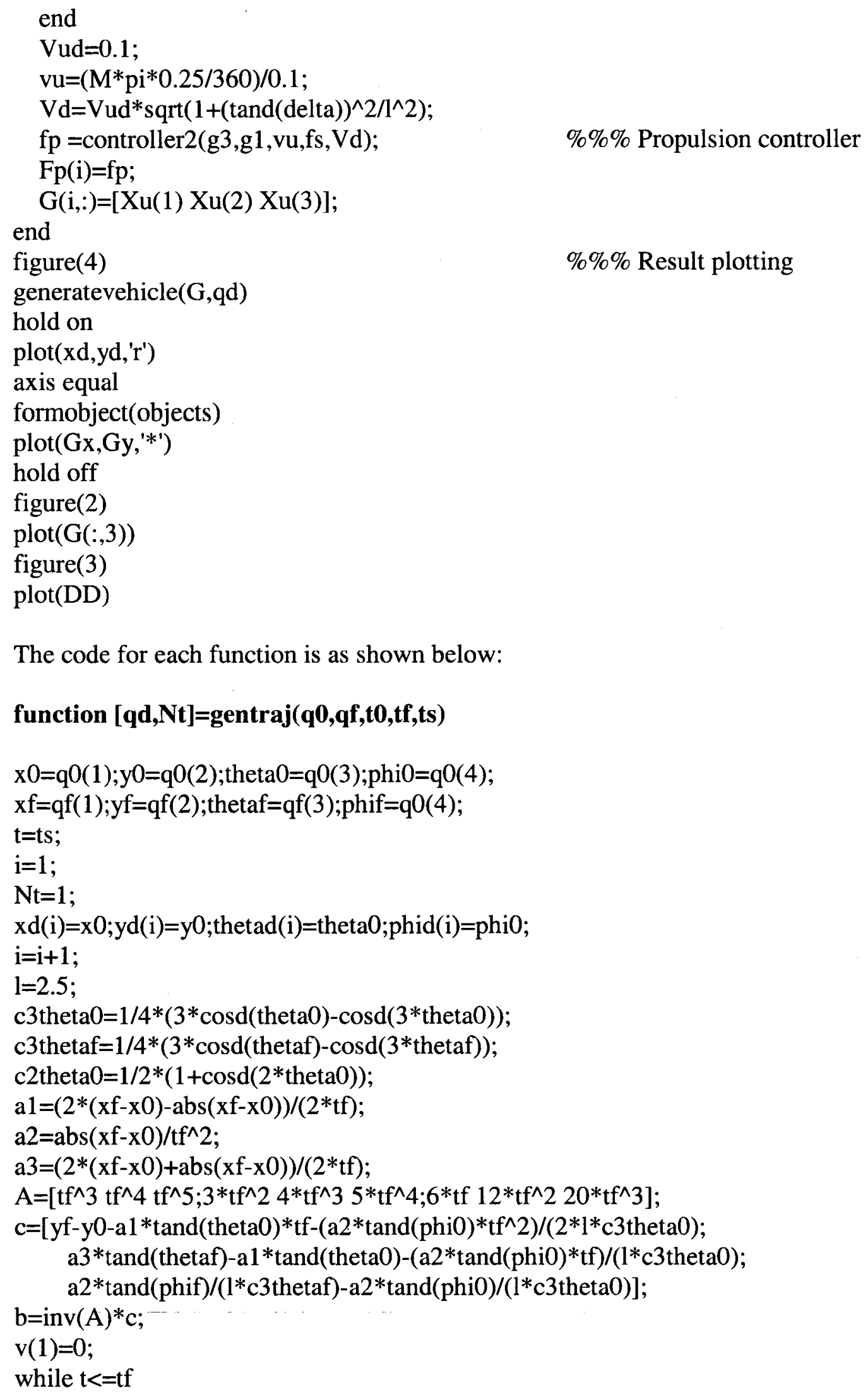

The code for each function is as shown below:

\section{function $[q d, N t]=$ gentraj $(q 0, q f, t 0, t f, t s)$}




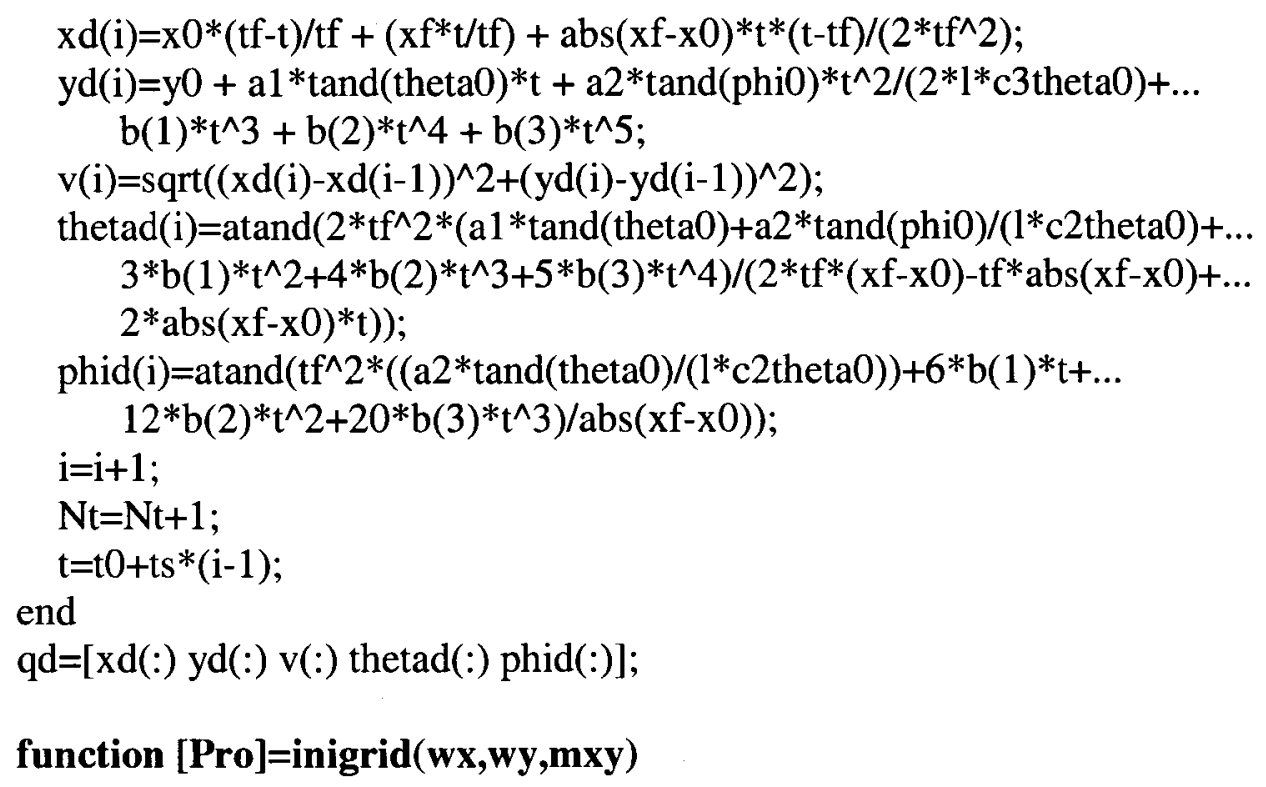

\section{function equation_line=objequations(objects,res)}

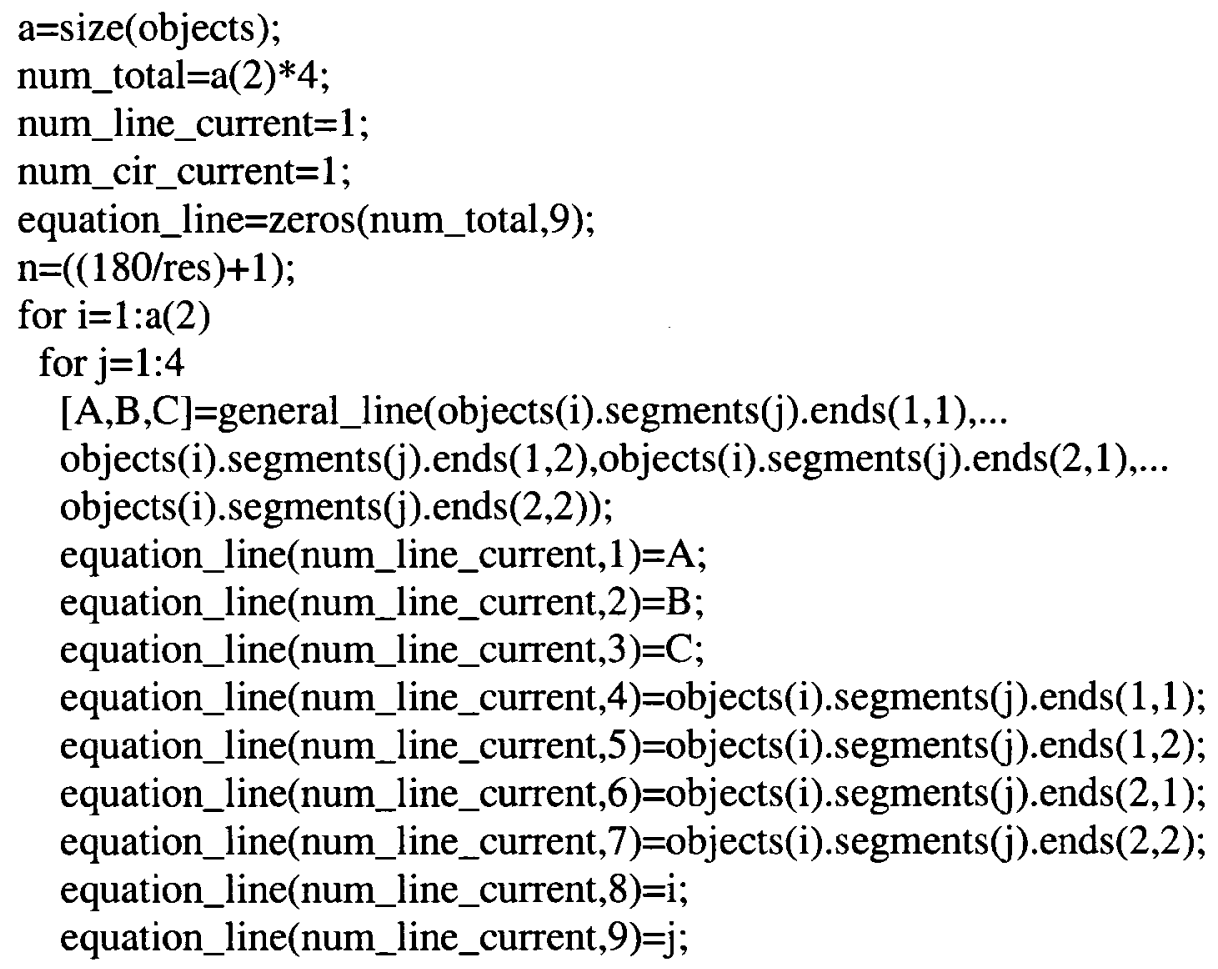


num_line_current=num_line_current +1 ;

end

end

\section{function $[\mathrm{D}, \mathrm{g1}, \mathrm{g3}]=\operatorname{dmode}(\mathrm{Vu}, \mathbf{f p}, \mathbf{f s}$, delta $)$}

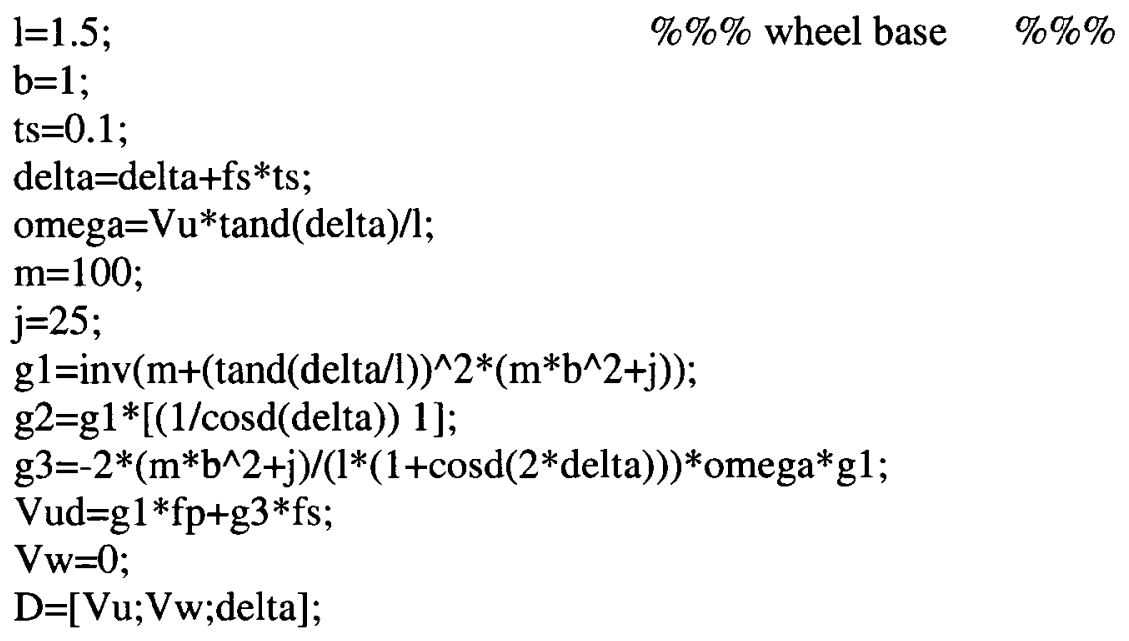

\section{function $[\mathrm{C}]=\mathrm{kmodel}(\mathrm{Vu}, \mathrm{Vw}, \operatorname{delta}, \mathrm{X})$}

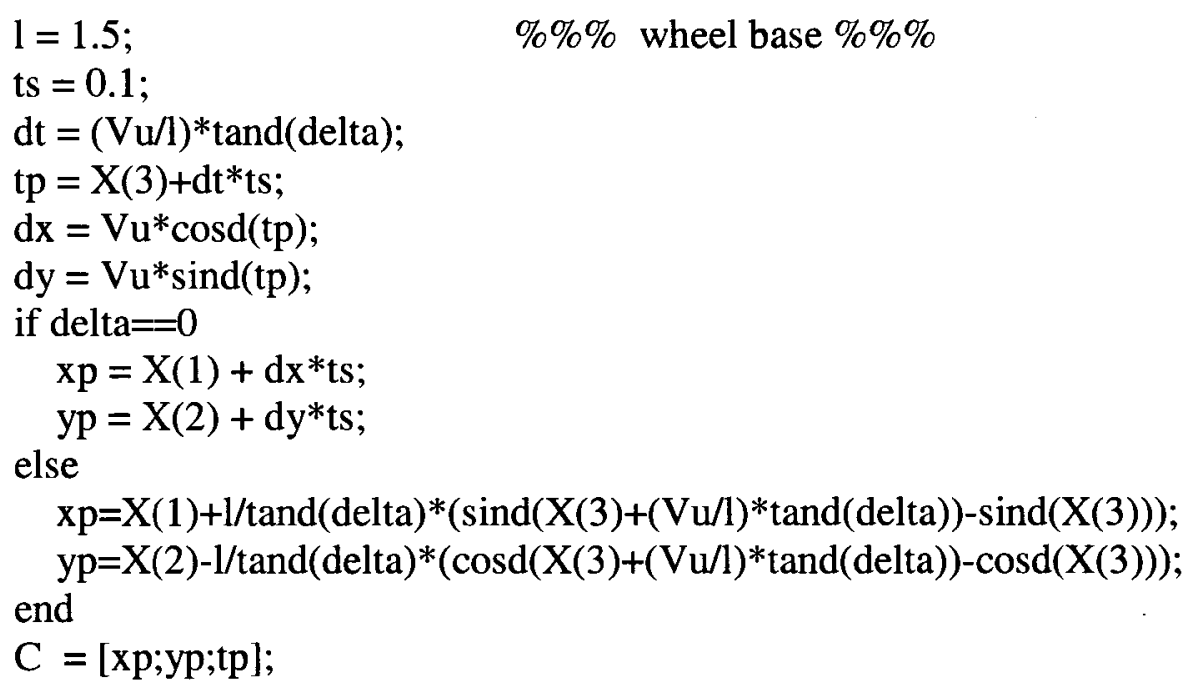

\section{function $[E]=$ priori $(\mathrm{Vu}, \mathrm{Vw}, \mathrm{X}, \mathrm{P})$}

$\mathrm{Q}=\operatorname{eye}(3)$;

$\mathrm{l}=1.5 ; \quad \% \% \%$ wheel base $\% \% \%$

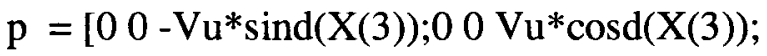

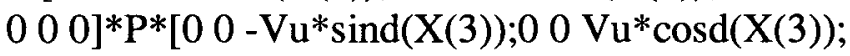

$\left.\begin{array}{lll}0 & 0 & 0\end{array}\right]^{\prime}+Q$

$\mathrm{E}=[\operatorname{reshape}(\mathrm{p}, 9,1)]$; 
end

end

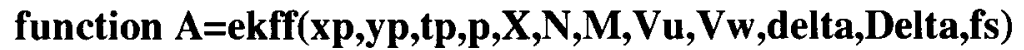

ts $=0.1$;

$\mathrm{x}=[\mathrm{xp} ; \mathrm{yp} ; \mathrm{tp}]$;

$\mathrm{D}=0.25$

$\mathrm{d}=0.025$;

$\mathrm{l}=0.15$;

$\% \% \%$ wheel base $\% \% \%$

$\mathrm{ds}=\mathrm{M}^{*} \mathrm{pi}^{*} \mathrm{D} / 360$

$\% \% \% \mathrm{D}=$ dia of wheel, $360=$ pulses;

$\mathrm{da}=\mathrm{N} / 10$;

$\% \% \% \mathrm{~d}=$ dia of steering rod, $360=$ pulses;

$\% \%$ predicted noiseless output from predicted states $\% \%$

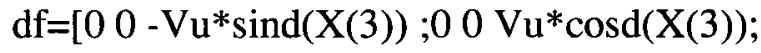

$0000]$;

$\mathrm{dh}=\mathrm{eye}(3)$;

$\mathrm{Q}=10 *$ eye (3);

$\mathrm{R}=500 *$ eye (3);

$\%$ ekf gain and error

$\mathrm{K}=\mathrm{p} * \mathrm{dh} \mathrm{h}^{*} \mathrm{inv}(\mathrm{dh} * \mathrm{p} * \mathrm{dh}+\mathrm{R})$;

if $\mathrm{da}==0$

$\mathrm{Dt}=\mathrm{Vu} * \operatorname{tand}($ delta $) / \mathrm{l}$

$\mathrm{tp}=\mathrm{X}(3)+\mathrm{Dt} * \mathrm{ts}$;

$\mathrm{Dx}=\mathrm{Vu} * \operatorname{cosd}(\mathrm{tp})$;

$\mathrm{Dy}=\mathrm{Vu} * \operatorname{sind}(\mathrm{tp})$

else

$\mathrm{Dx}=\mathrm{ds} /(\mathrm{da}) * \cos d($ Delta $) *(\operatorname{cosd}(\mathrm{da})-1)-\mathrm{ds} /(\mathrm{da}) * \operatorname{sind}(\mathrm{da}) * \operatorname{sind}($ Delta $)$;

$\mathrm{Dy}=\mathrm{ds} /(\mathrm{da}) * \operatorname{sind}($ Delta $) *(\operatorname{cosd}(\mathrm{da})-1)+\mathrm{ds} /(\mathrm{da}) * \operatorname{sind}(\mathrm{da}) * \operatorname{cosd}($ Delta $)$;

$\mathrm{Dt}=\mathrm{Vu} * \operatorname{tand}($ Delta $) / \mathrm{l}$;

end

$\mathrm{Z}=[\mathrm{X}(1)+\mathrm{Dx} ; \mathrm{X}(2)+\mathrm{Dy} ; \mathrm{X}(3)+\mathrm{Dt}]$;

$\mathrm{hx}=\mathrm{dh} * \mathrm{x}$;

e=Z-hx;

$\%$ Updates-

$\mathrm{Xu}=\mathrm{x}+\mathrm{K} * \mathrm{e}$;

$\mathrm{Pu}=\left(\operatorname{eye}(3)-\mathrm{K}^{*} \mathrm{dh}\right) * \mathrm{p}$;

$\mathrm{P}=$ reshape $(\mathrm{Pu}, 9,1)$;

$\mathrm{A}=[\mathrm{Xu} ; \mathrm{P}]$;

function [y,count]=iscolission(filter,num_points)

$y=-1 ;$ 


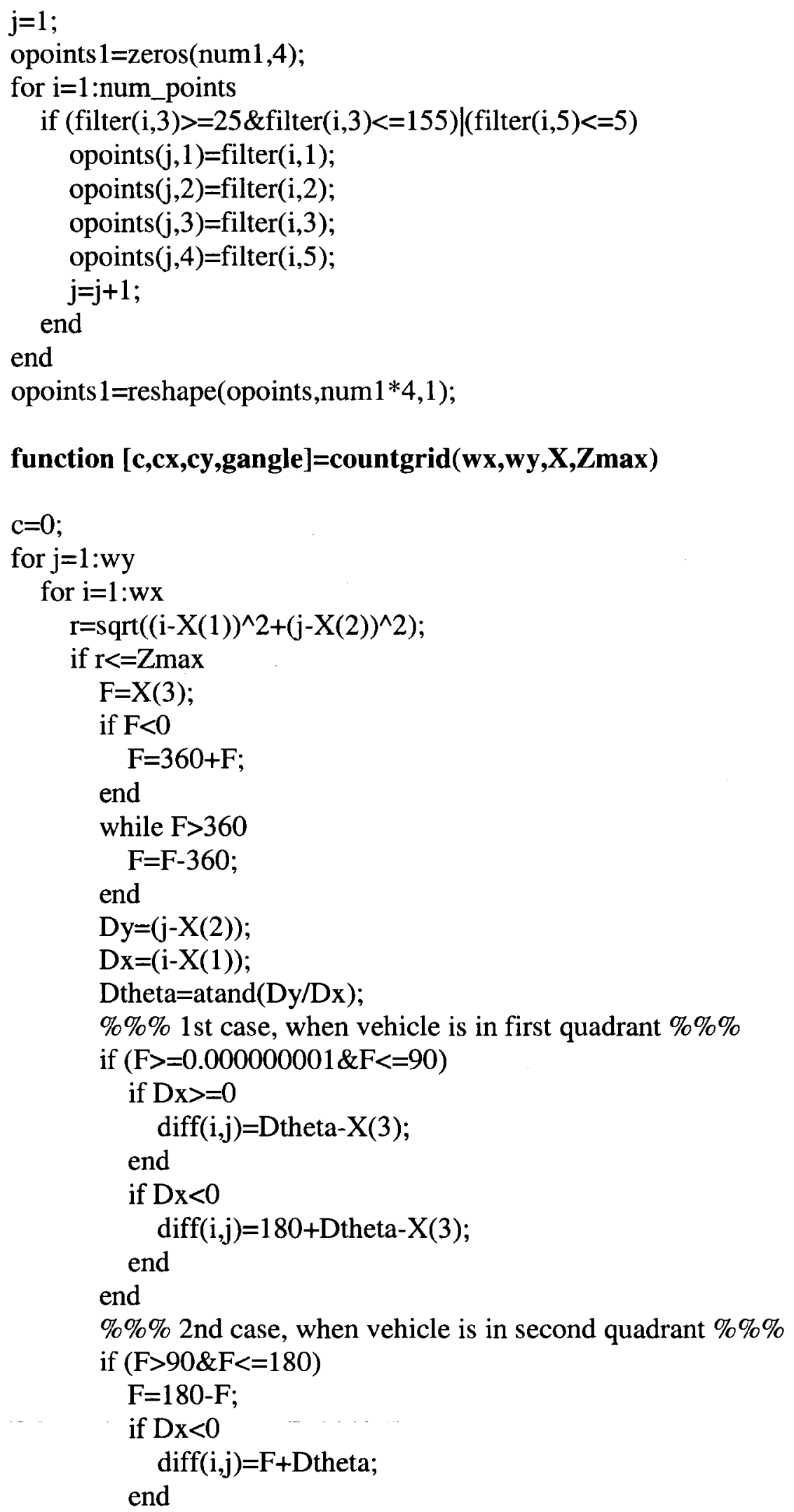




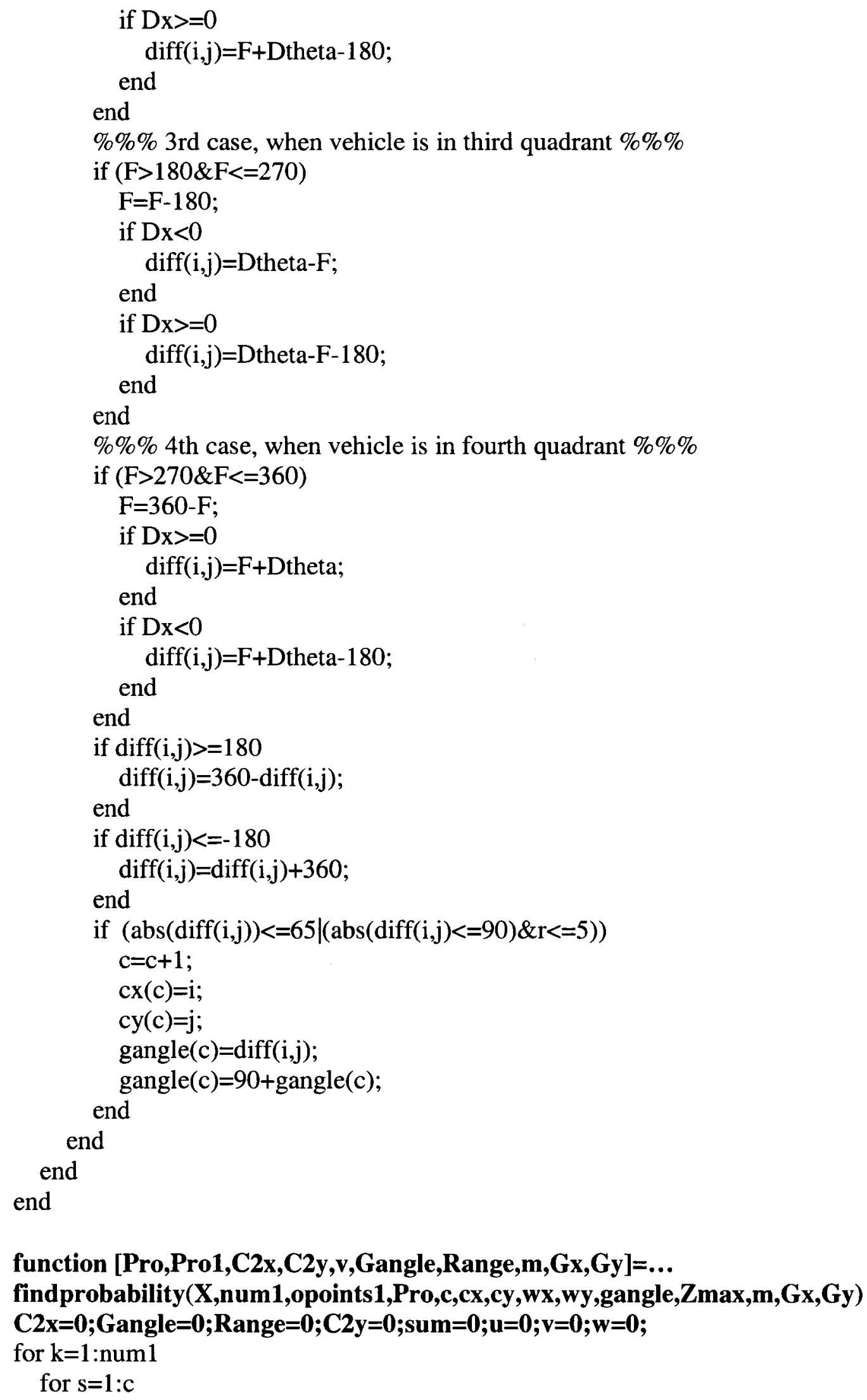




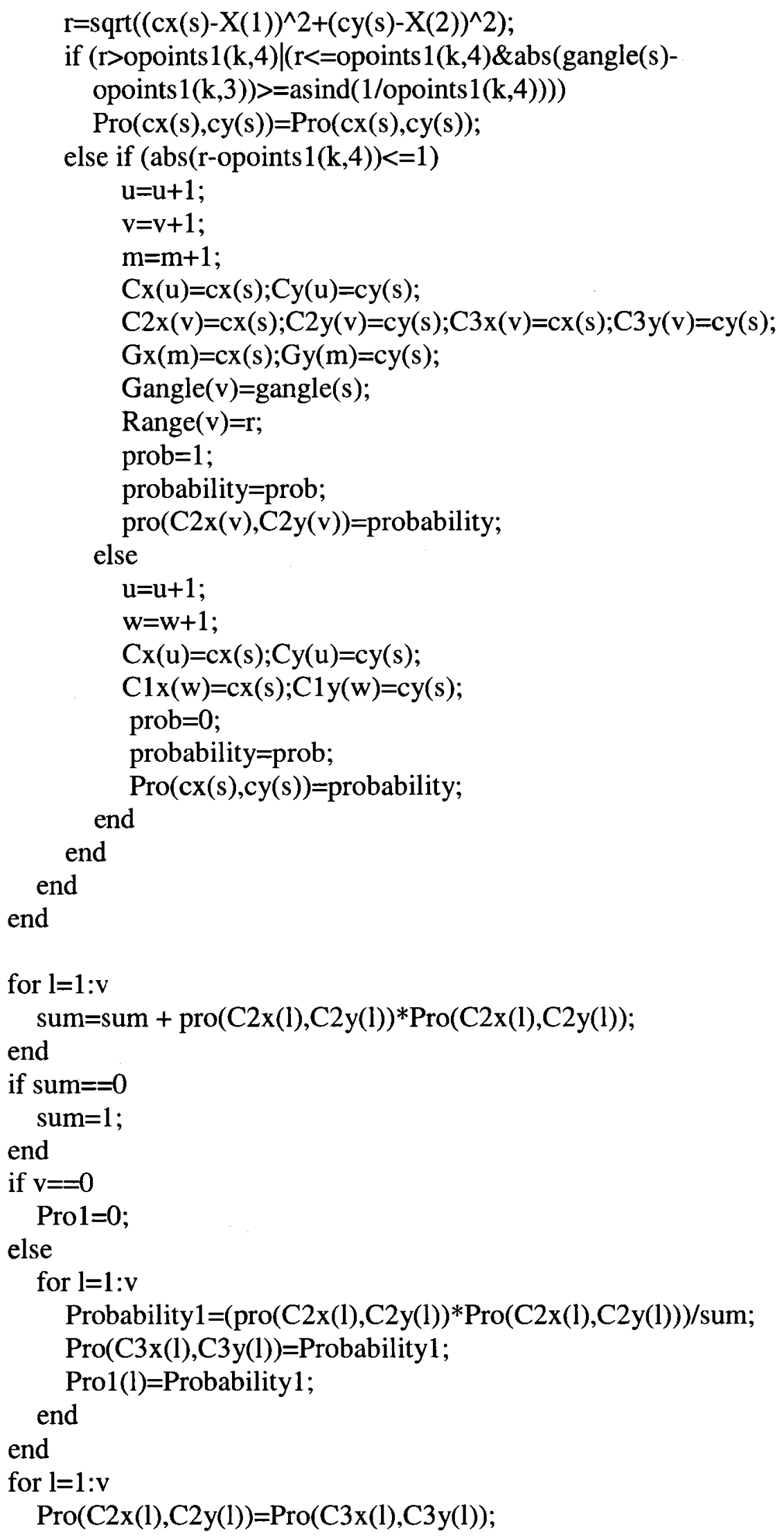


end

\section{function [Opoints]=occgridpoints(C2x,C2y,v,Gangle,Range)}

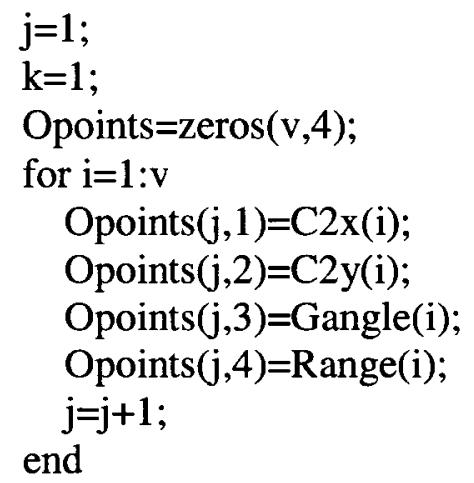

\section{function [cpoints]=colpoints(num1,opoints,Nobjects,di)}

cpoints=zeros(Nobjects, 8$)$;

if numl $1==1$

cpoints $(1,1)=$ opoints $(1,1)$;

cpoints $(1,2)=$ opoints $(1,2)$;

cpoints $(1,3)=0$;

cpoints $(1,4)=0$;

cpoints $(1,5)=$ opoints $(1,3)$;

cpoints $(1,6)=0$;

cpoints $(1,7)=$ opoints $(1,4)$; $\operatorname{cpoints}(1,8)=0$

else $\mathrm{i}=1 ; \mathrm{j}=1$; 


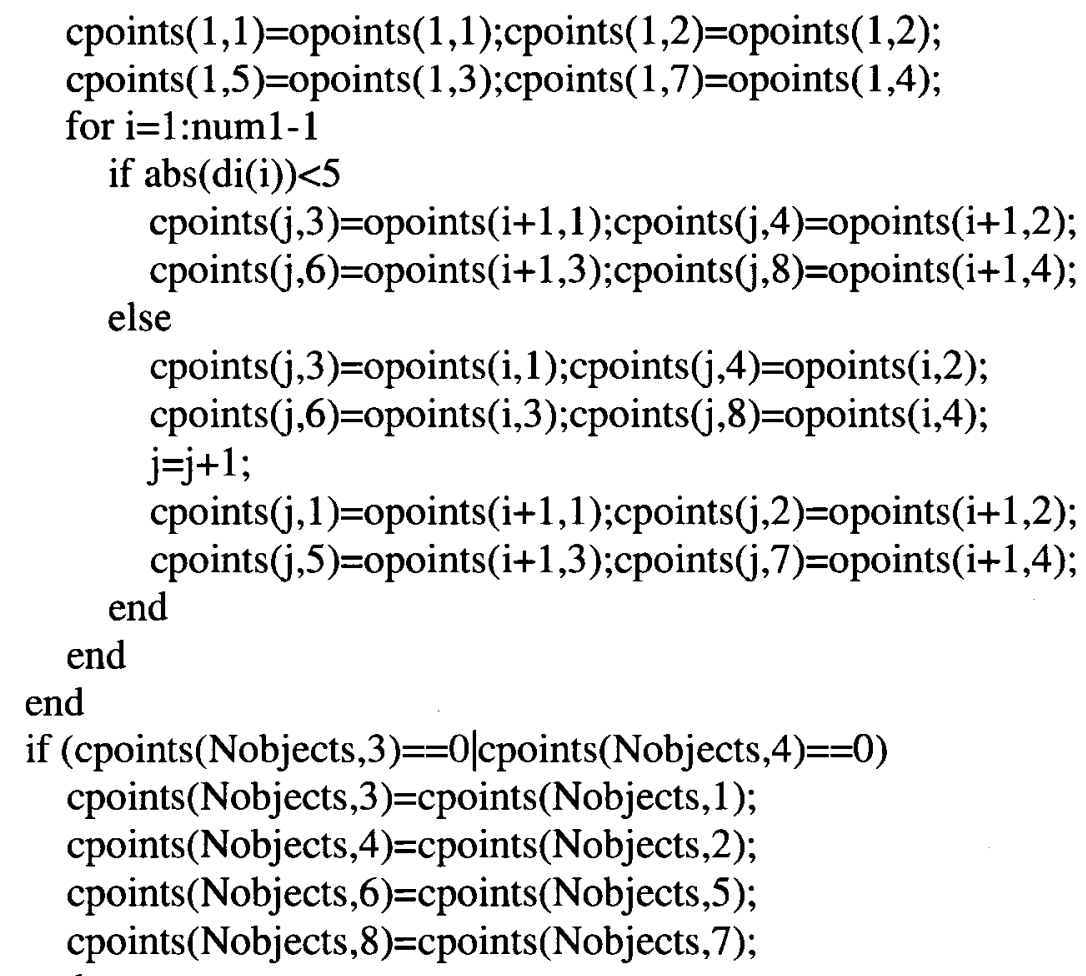

function [X_t,Y_t,T_d,Dtheta]=reqtheta(X,Xd,yd,thetad,cpoints,Nt,Nobjects)

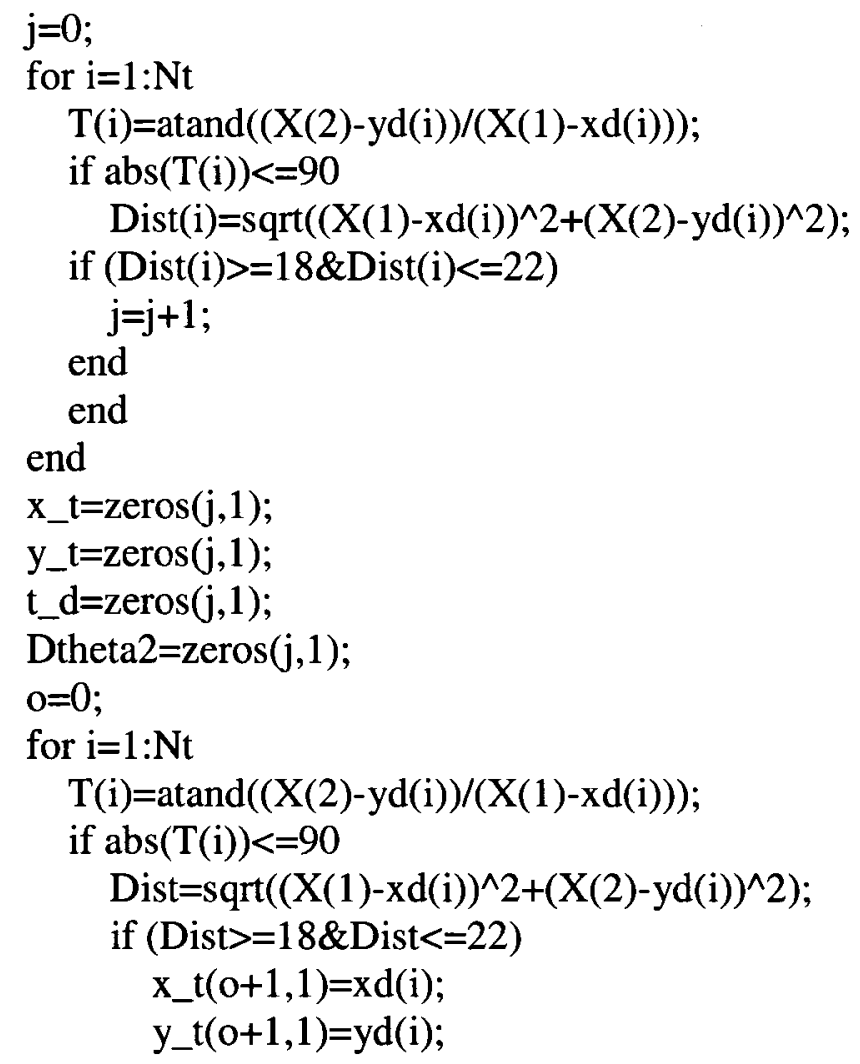




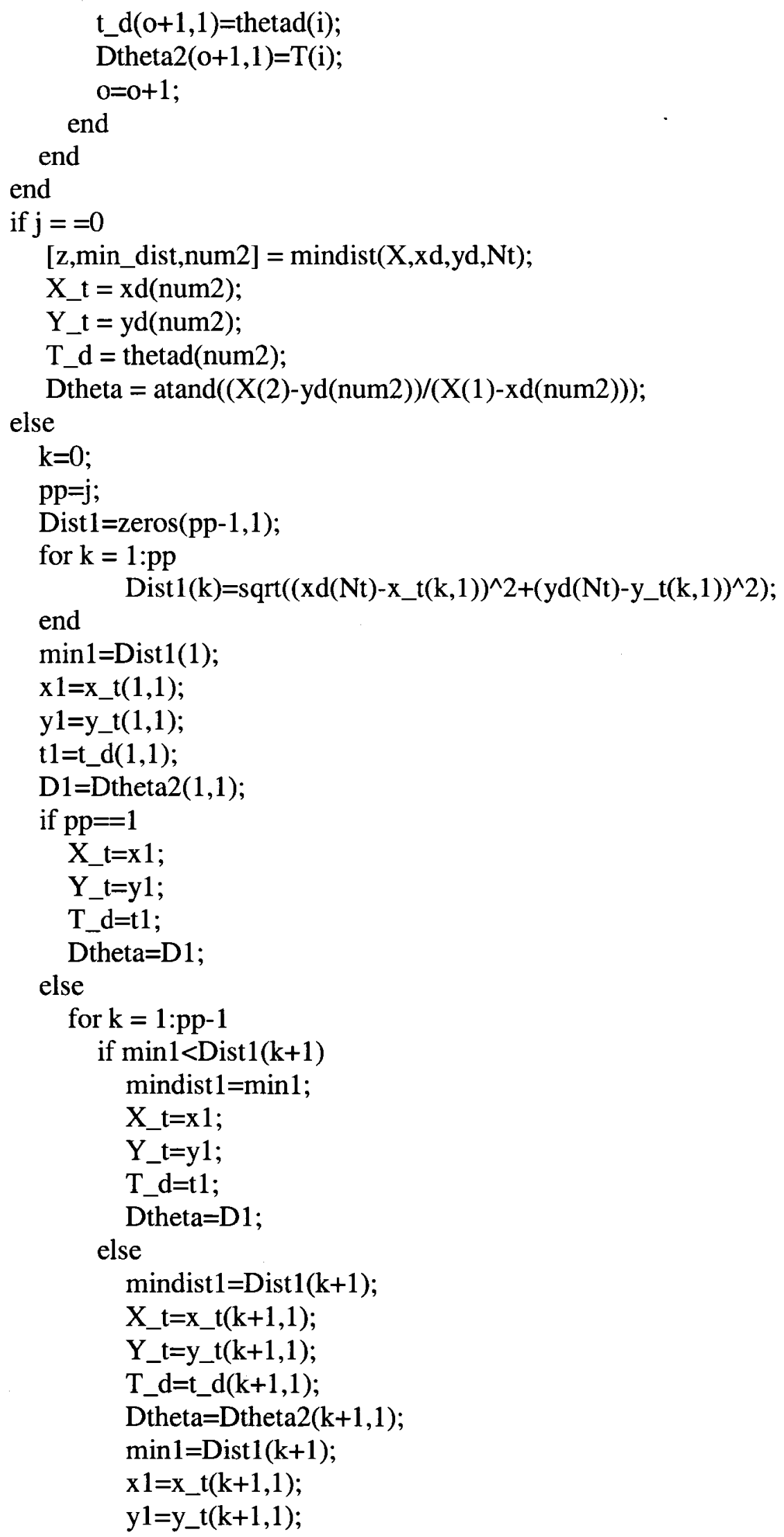




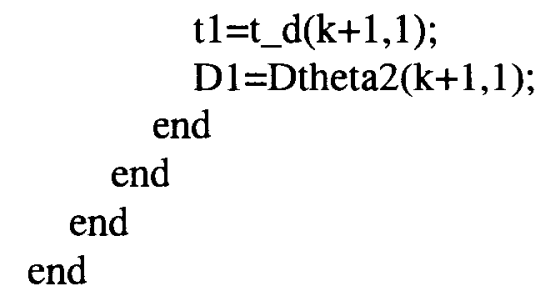

\section{function $[$ des,fs] $=$ finddeltao3...}

(dl,dc,dr,num1,num_points,X_t,Y_t,Dtheta,opoints,cpoints,Nobjects,l,X,des,xd,yd,th etad,Nt,nu,Delta,delta,count)

followtraj $=0$;

avoidcollision $=0$;

$F=X(3)$;

if $\mathrm{F}<0$

$F=360+F$;

end

if $\mathrm{F}>360$

$\mathrm{F}=\mathrm{F}-360$;

end

$\mathrm{Dy}=\left(\mathrm{Y}_{-} \mathrm{t}-\mathrm{X}(2)\right)$;

$\mathrm{Dx}=\left(\mathrm{X}_{-} \mathrm{t}-\mathrm{X}(1)\right)$;

$\mathrm{k}=0$;

for $\mathrm{i}=1$ :num 1

if opoints $(\mathrm{i}, 4)<=3$

$\mathrm{k}=\mathrm{k}+1$;

$\mathrm{nx}(\mathrm{k})=$ opoints $(\mathrm{i}, 1)$;

ny(k)=opoints $(\mathrm{i}, 2)$;

$n t(k)=$ opoints $(\mathrm{i}, 3)$;

$\operatorname{nr}(\mathrm{k})=$ opoints $(\mathrm{i}, 4)$;

end

end

$\% \% \% 1$ st case, when vehicle is in first quadrant $\% \% \%$

if $(\mathrm{F}>=0.000000001 \& \mathrm{~F}<=90)$

if $\mathrm{Dx}>=0$

dd2=Dtheta-X(3);

end

if $\mathrm{Dx}<0$

$\mathrm{dd} 2=180+$ Dtheta-X(3);

end

end

$\% \% \%$ 2nd case, when vehicle is in second quadrant $\% \% \%$

if $(\mathrm{F}>90 \& \mathrm{~F}<=180)$

$\mathrm{F}=180-\mathrm{F}$; 


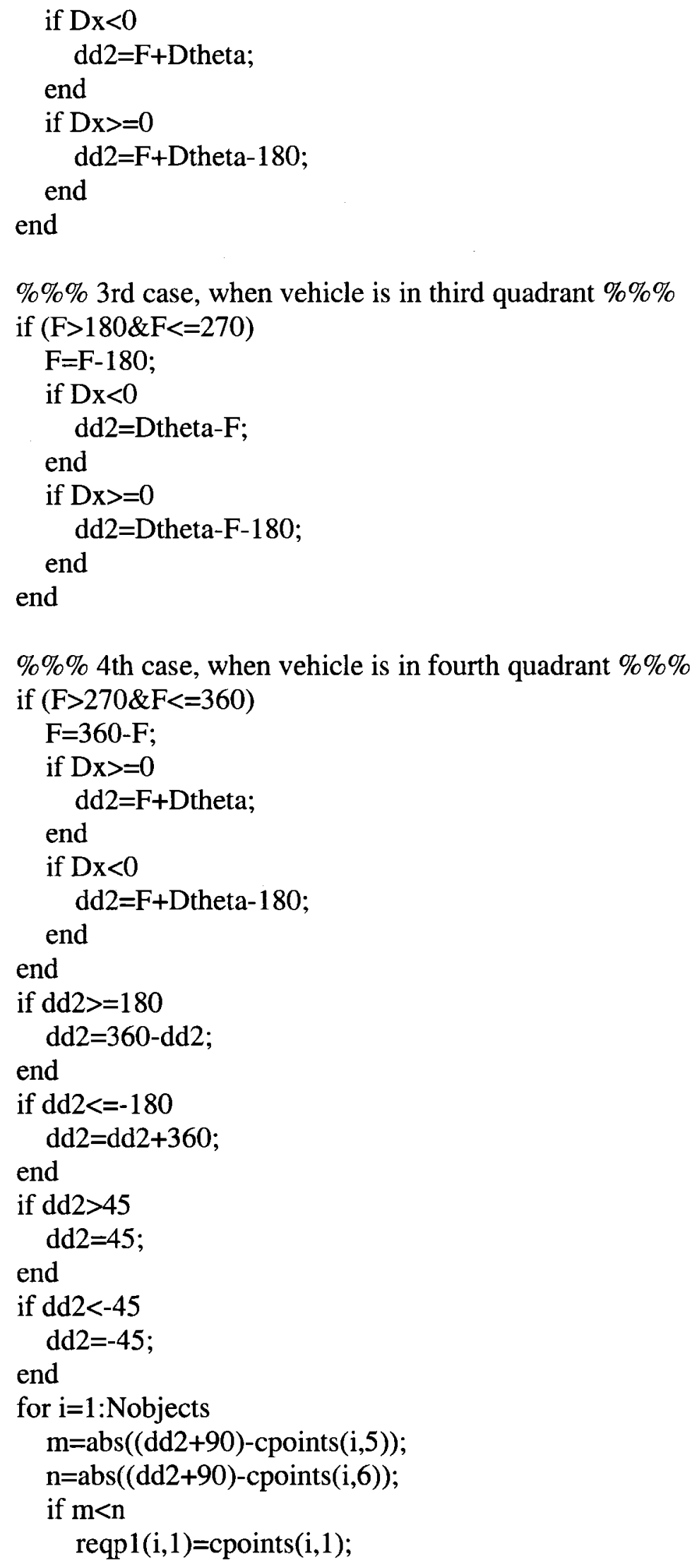




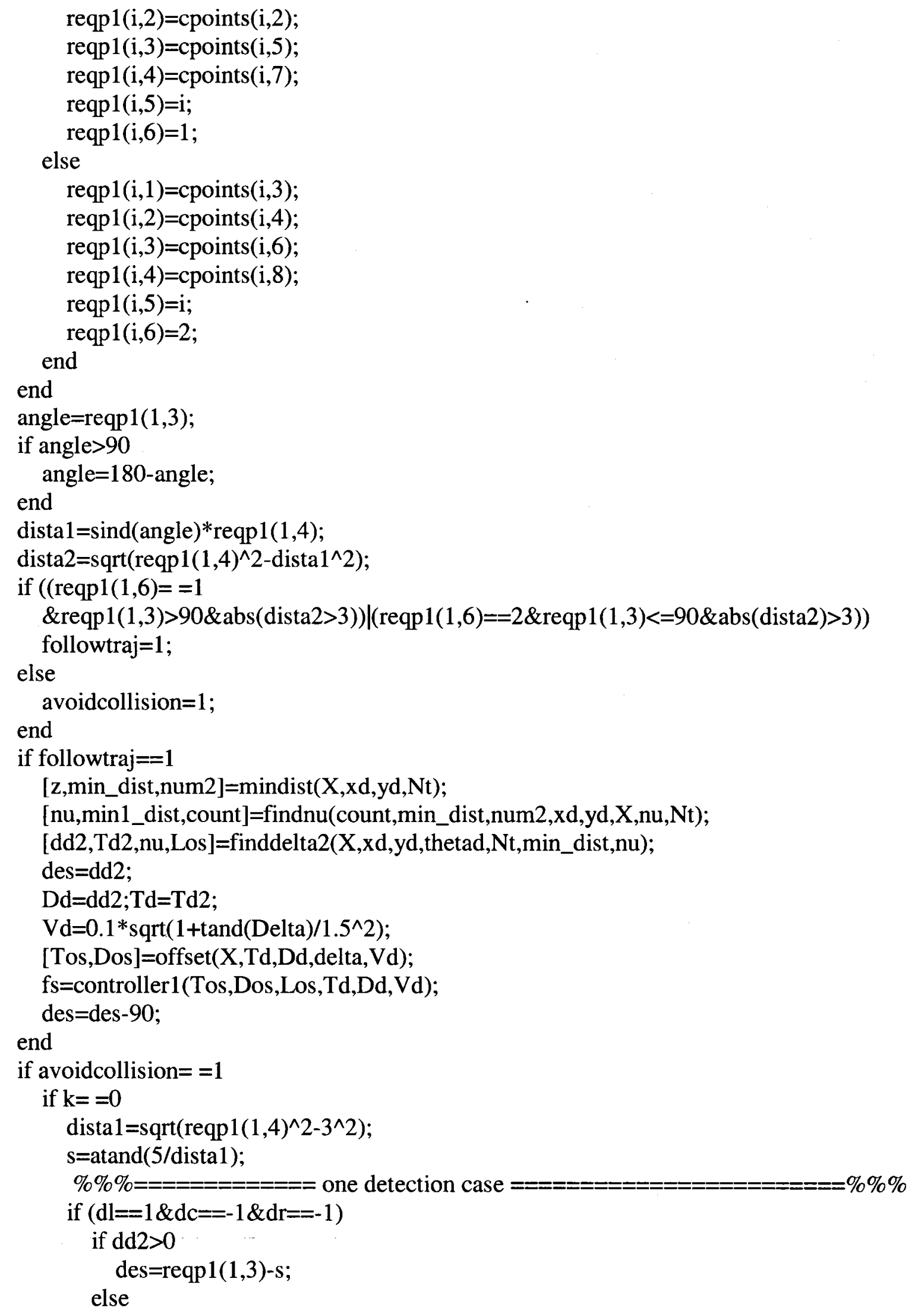




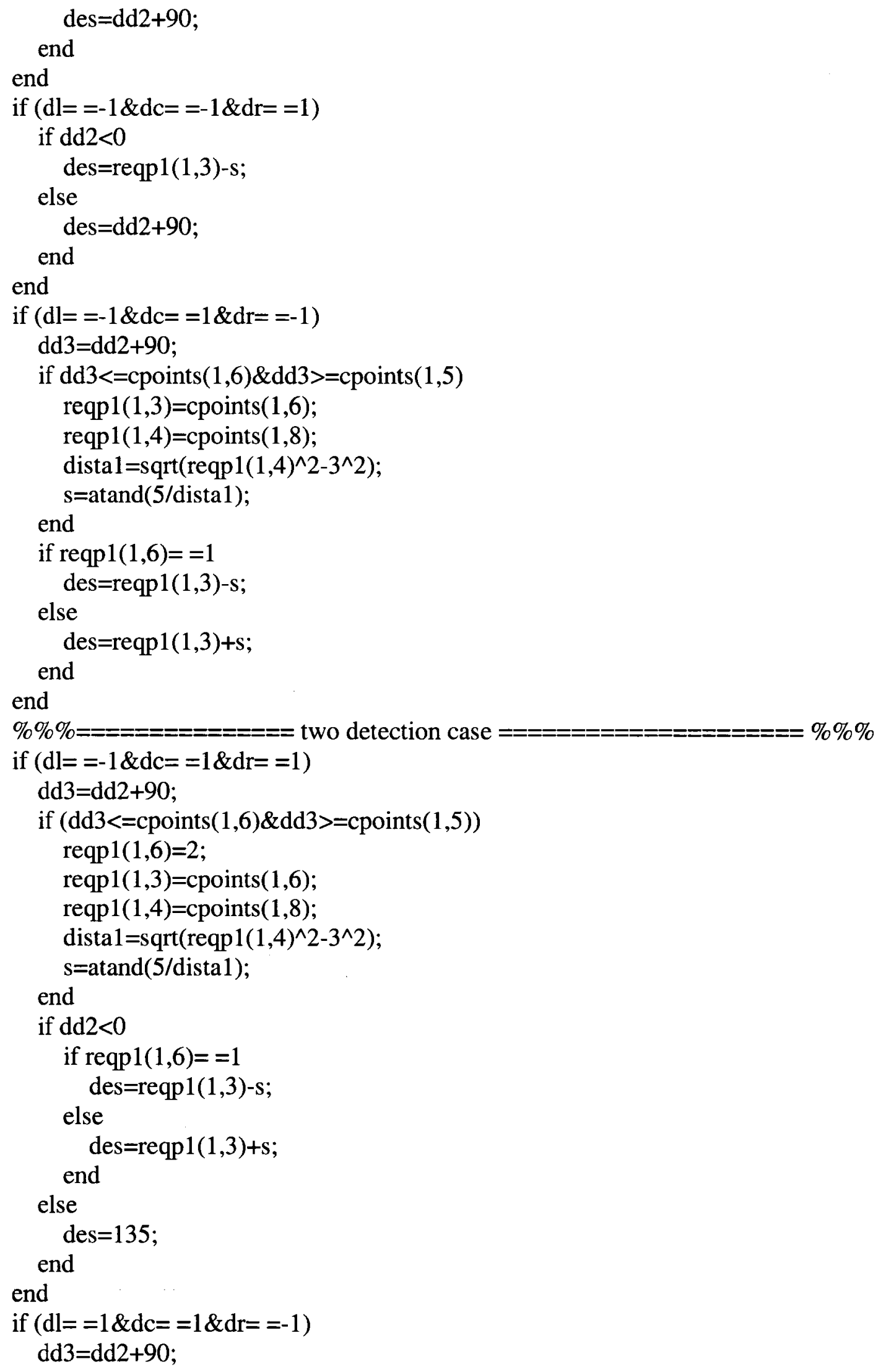




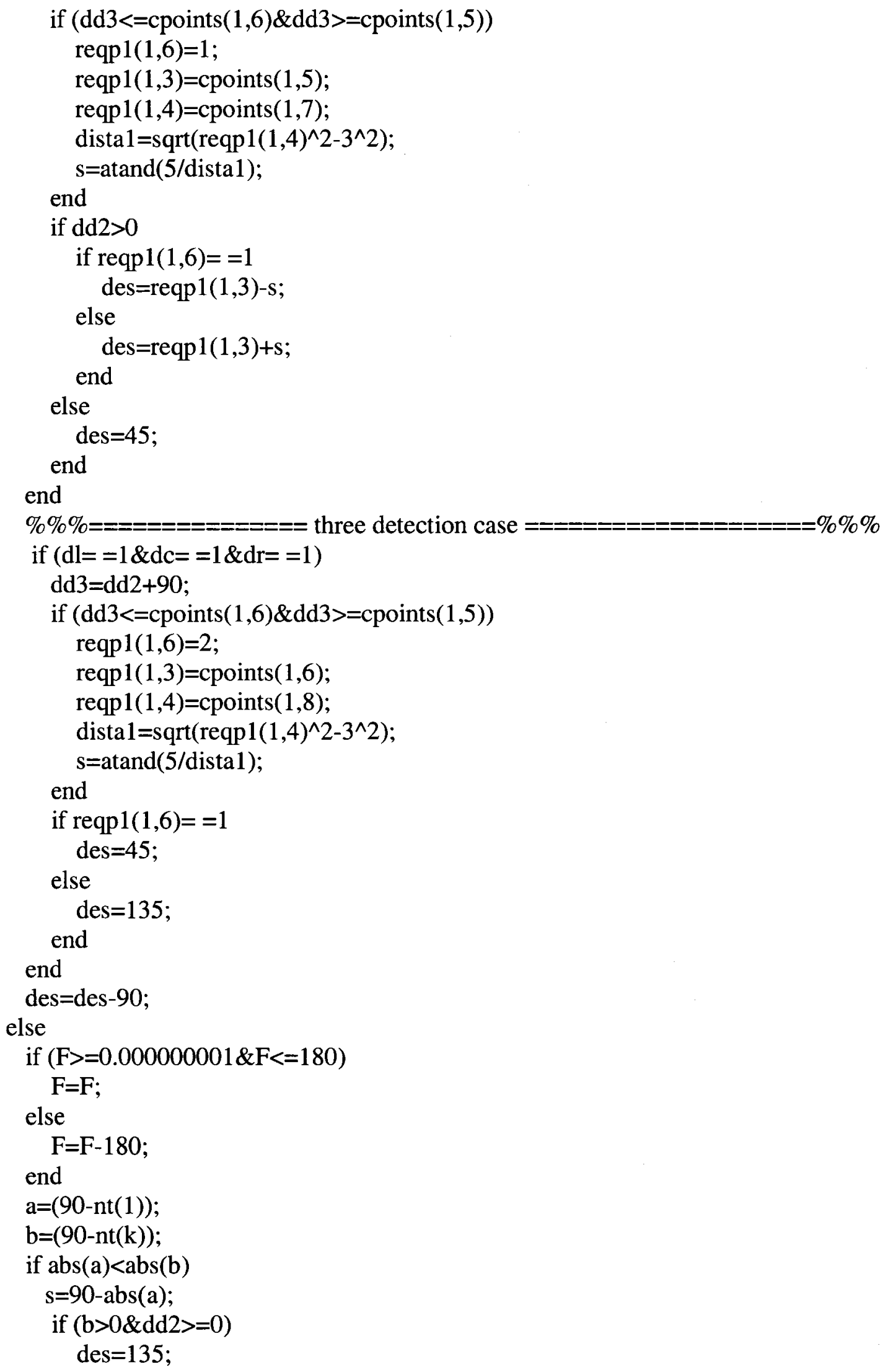




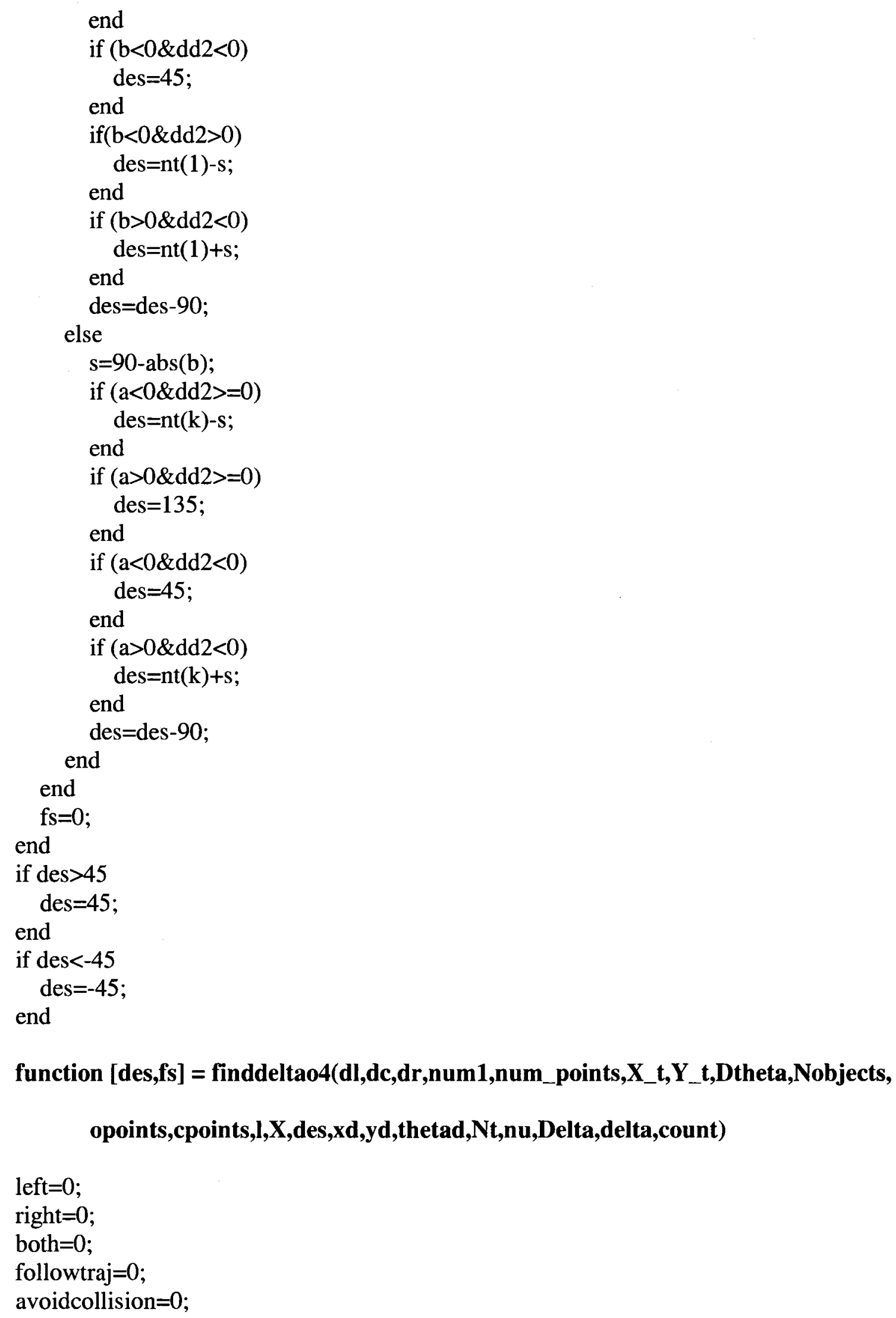

function [des,fs] = finddeltao4(dl,dc,dr,num1,num_points,X_t,Y_t,Dtheta,Nobjects, opoints,cpoints,l,X,des,Xd,yd,thetad,Nt,nu,Delta,delta,count)

left $=0$;

right $=0$;

both $=0$;

followtraj $=0$;

avoidcollision $=0$; 


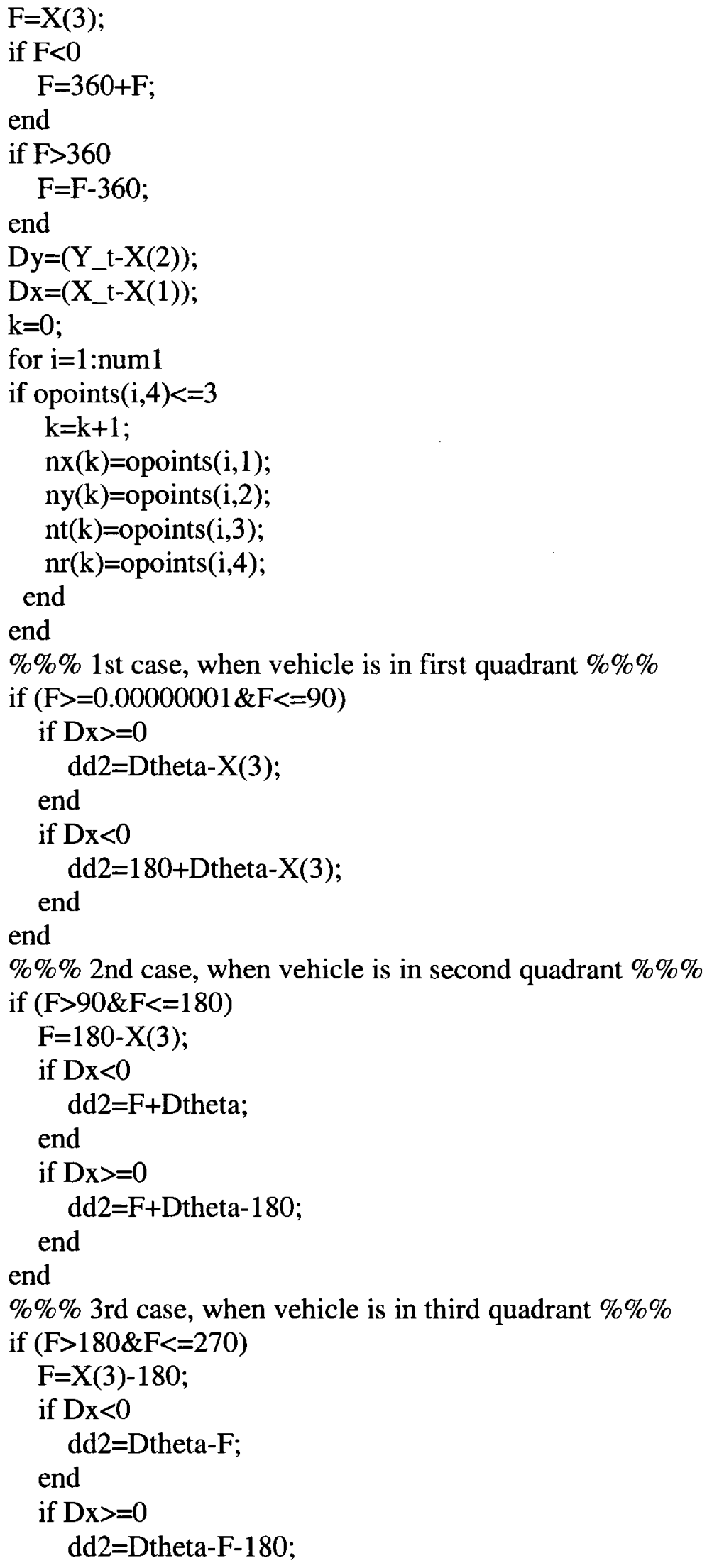




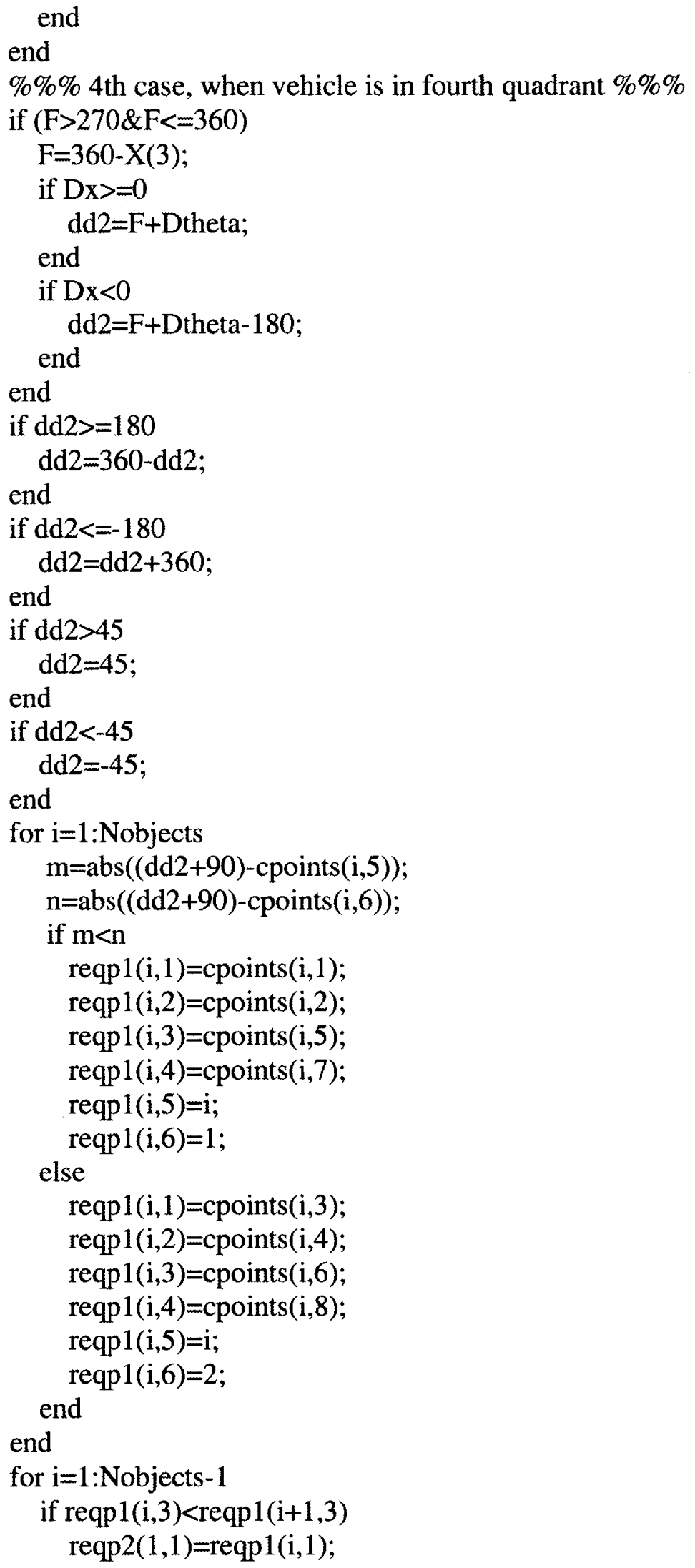




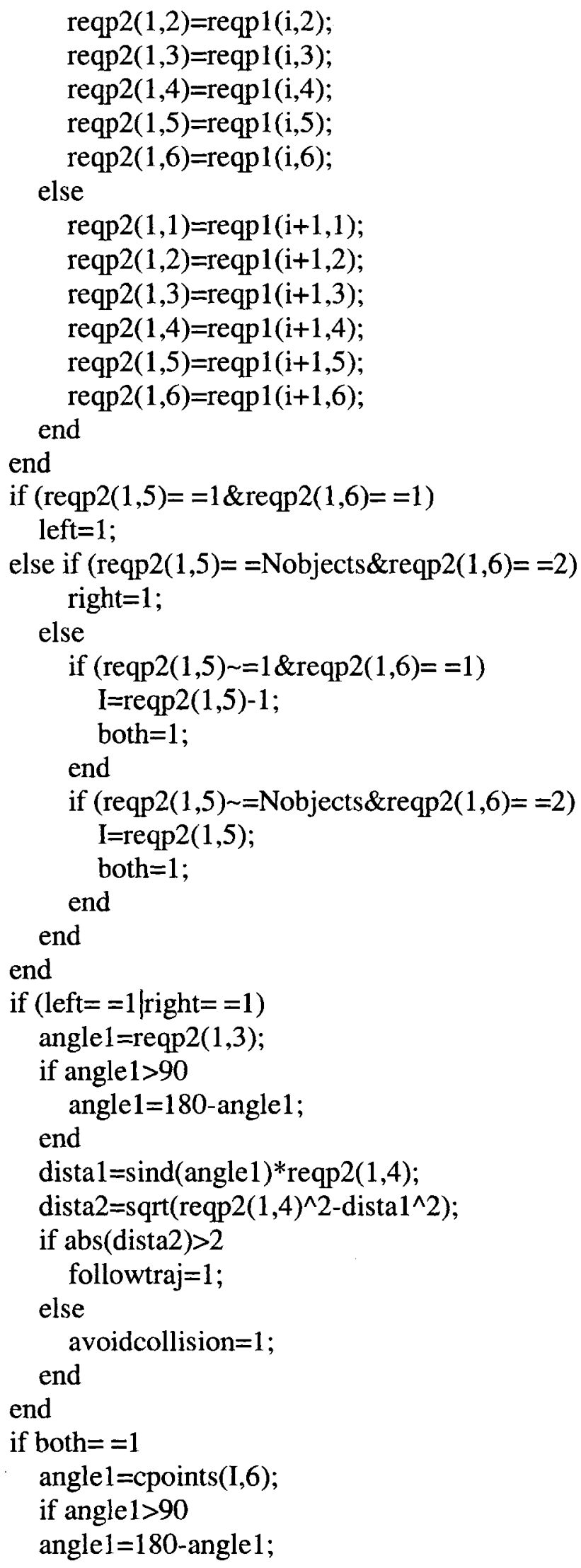


end

angle $2=\operatorname{cpoints}(I+1,5)$;

if angle $2>90$

angle2=180-angle2;

end

dista $1=\operatorname{sind}($ angle1 $) *$ cpoints $(\mathrm{I}, 8) ;$ dista $2=\operatorname{sind}($ angle 2$) * \operatorname{cpoints}(\mathrm{I}+1,7)$;

dista3 $=\operatorname{sqrt}\left(\operatorname{cpoints}(I, 8)^{\wedge} 2-\operatorname{dista}^{\wedge}{ }^{\wedge} 2\right) ; \operatorname{dista} 4=\operatorname{sqrt}\left(\operatorname{cpoints}(I+1,7)^{\wedge} 2-\operatorname{dista} 2^{\wedge} 2\right)$;

if $\operatorname{abs}(($ dista3 $)>3 \& \operatorname{abs}($ dista4 $)>3$ )

followtraj $=1$;

else

avoidcollision=1;

end

end

if followtraj==1

$\left[z, \min \_\right.$dist, num2 $]=\operatorname{mindist}(X, x d, y d, N t)$;

[nu,min1_dist,count]=findnu(count,min_dist,num2,xd,yd,X,nu,Nt);

[dd2,Td2,nu,Los]=finddelta2(X,Xd,yd,thetad,Nt,min_dist,nu);

des $=\mathrm{dd} 2$;

$\mathrm{Dd}=\mathrm{dd} 2 ; \mathrm{Td}=\mathrm{Td} 2$;

Vud $=0.1$;

$\mathrm{Vd}=\operatorname{Vud} * \operatorname{sqrt}\left(1+(\operatorname{tand}(\text { delta }))^{\wedge} 2 / 1^{\wedge} 2\right)$;

[Tos,Dos]=offset(X,Td,Dd,delta,Vud);

fs=controller 1 (Tos,Dos,Los,Td,Dd,Vd);

des=des -90 ;

end

if $\operatorname{reqp} 2(1,4)=0$

reqp $2(1,4)=10$;

end

if avoidcollision $==1$

if $\mathrm{k}==0$

count $1=0$;

distal $=\operatorname{sqrt}\left(\operatorname{reqp} 2(1,4)^{\wedge} 2-3^{\wedge} 2\right)$;

$\mathrm{s}=\operatorname{atand}(5 /$ dista 1$) ;$

$\% \% \%===========$ one detection case $=======================\% \% \%$

if $(\mathrm{dl}==1 \& \mathrm{dc}==-1 \& \mathrm{dr}==-1)$

if $\mathrm{dd} 2>0$

des=reqp2(1,3)-s;

else

des $=\mathrm{dd} 2+90$

end

end

if $(\mathrm{dl}==-1 \& \mathrm{dc}==-1 \& \mathrm{dr}==1)$

if $\mathrm{dd} 2<0$

des=reqp $2(1,3)+s$;

else

des $=\mathrm{dd} 2+90$ 


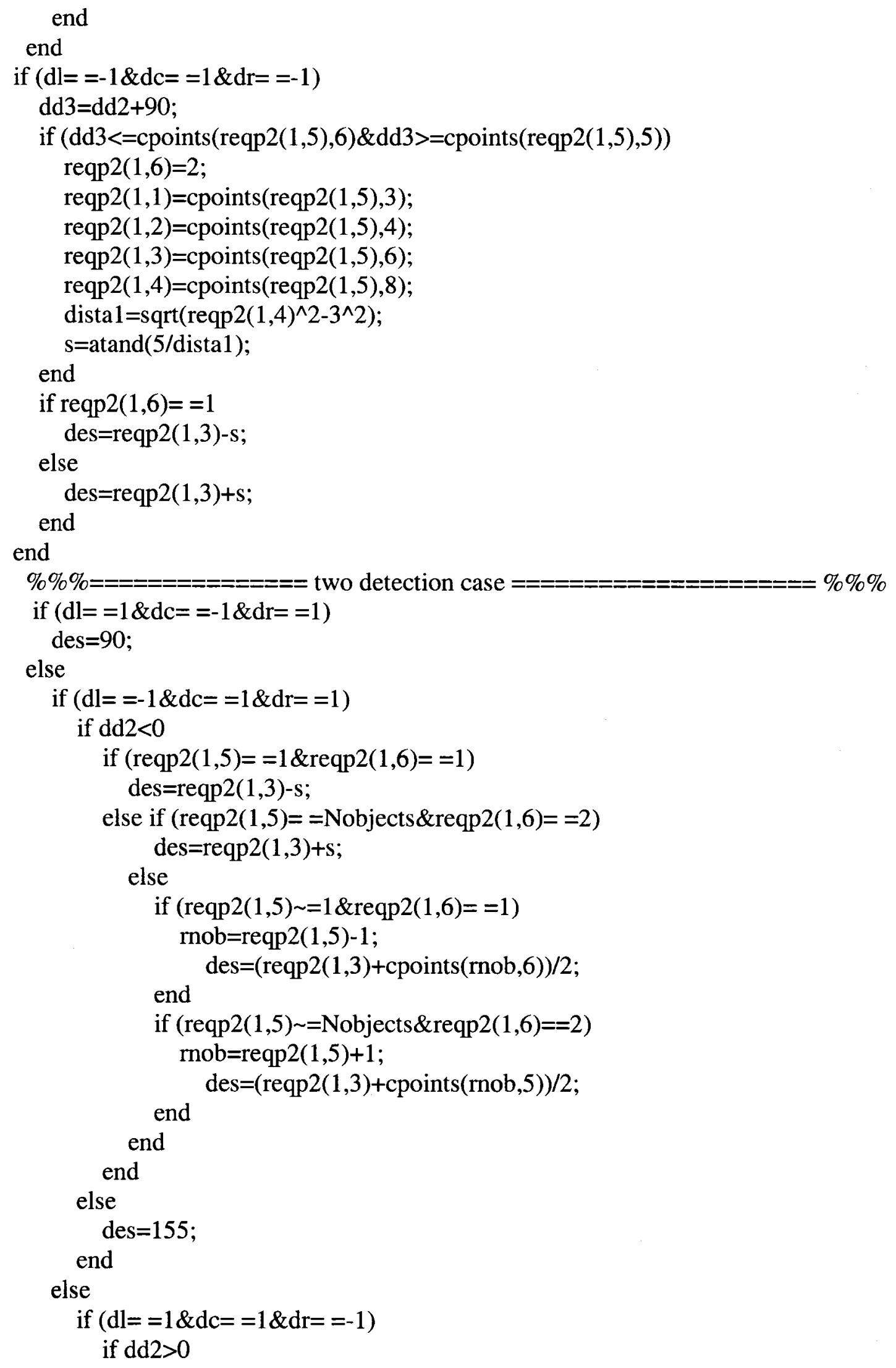




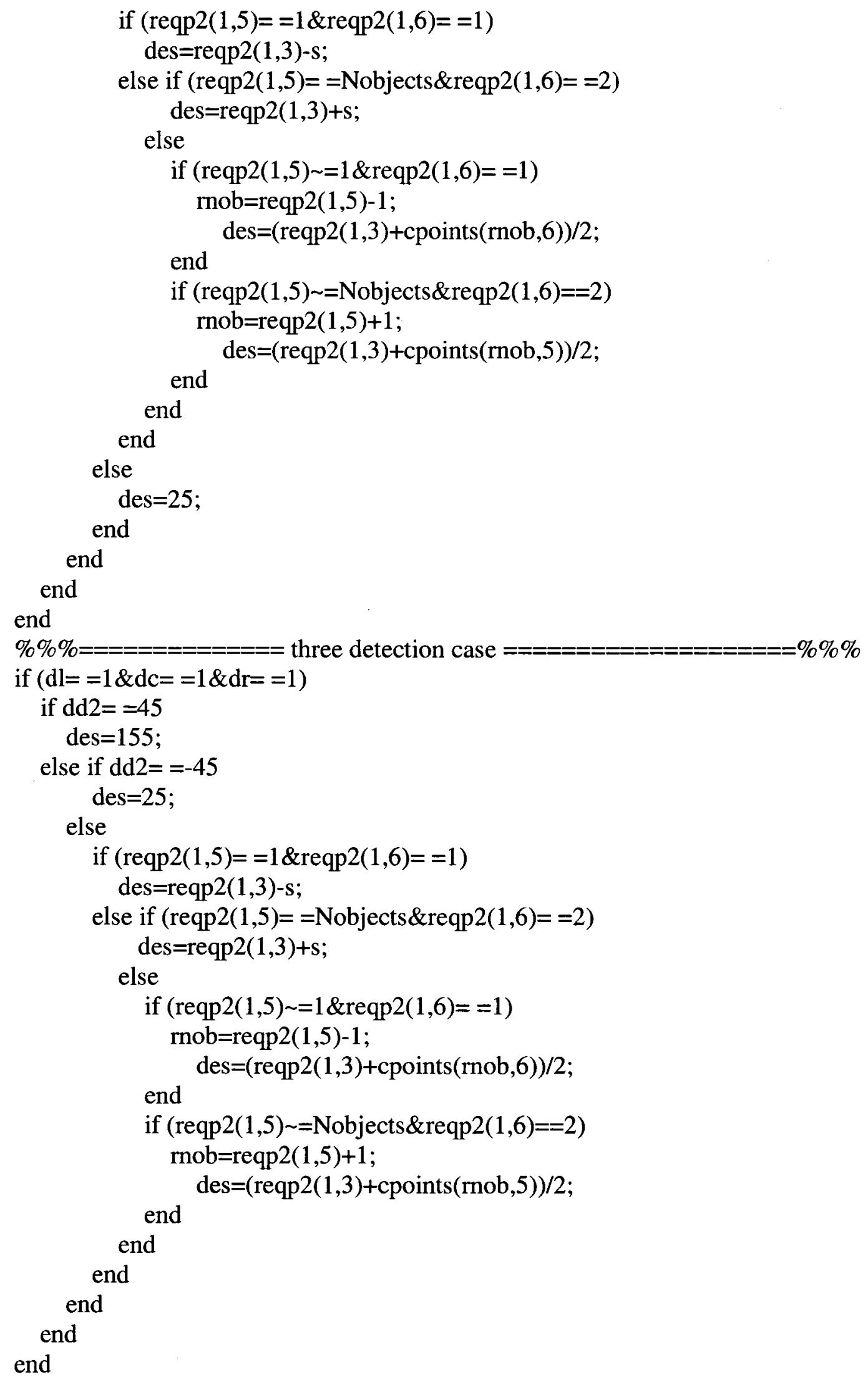




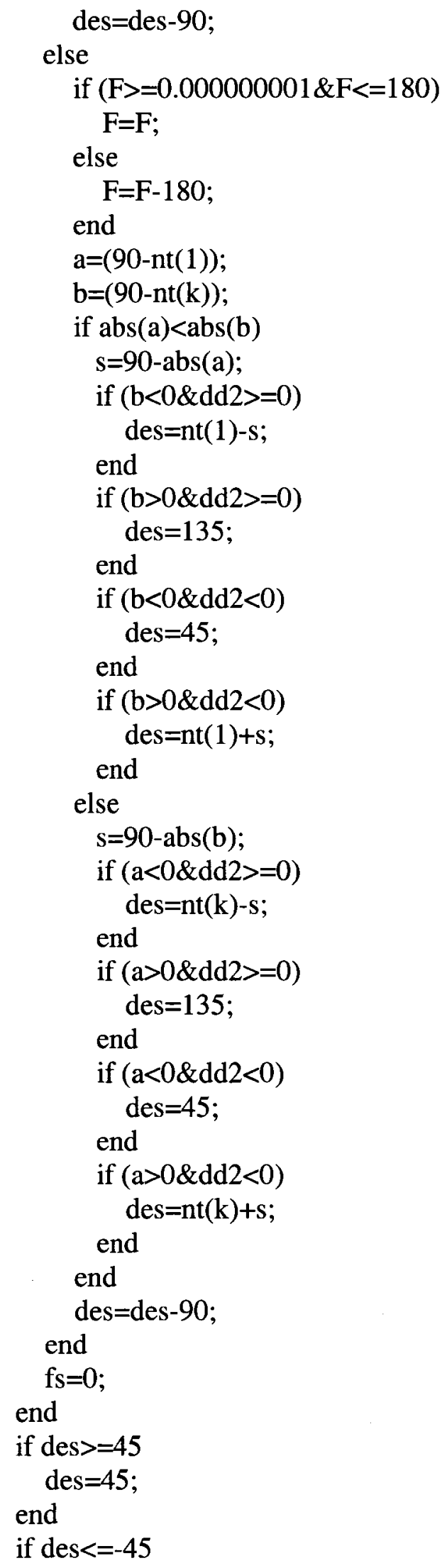


$\operatorname{des}=-45$

end

function [des,fs]=finddeltao4(dl,dc,dr,num1,num_points,X_t,Y_t,Dtheta,Nobjects,... opoints,cpoints,l,X,des,Xd,yd,thetad,Nt,nu,Delta,delta,count)

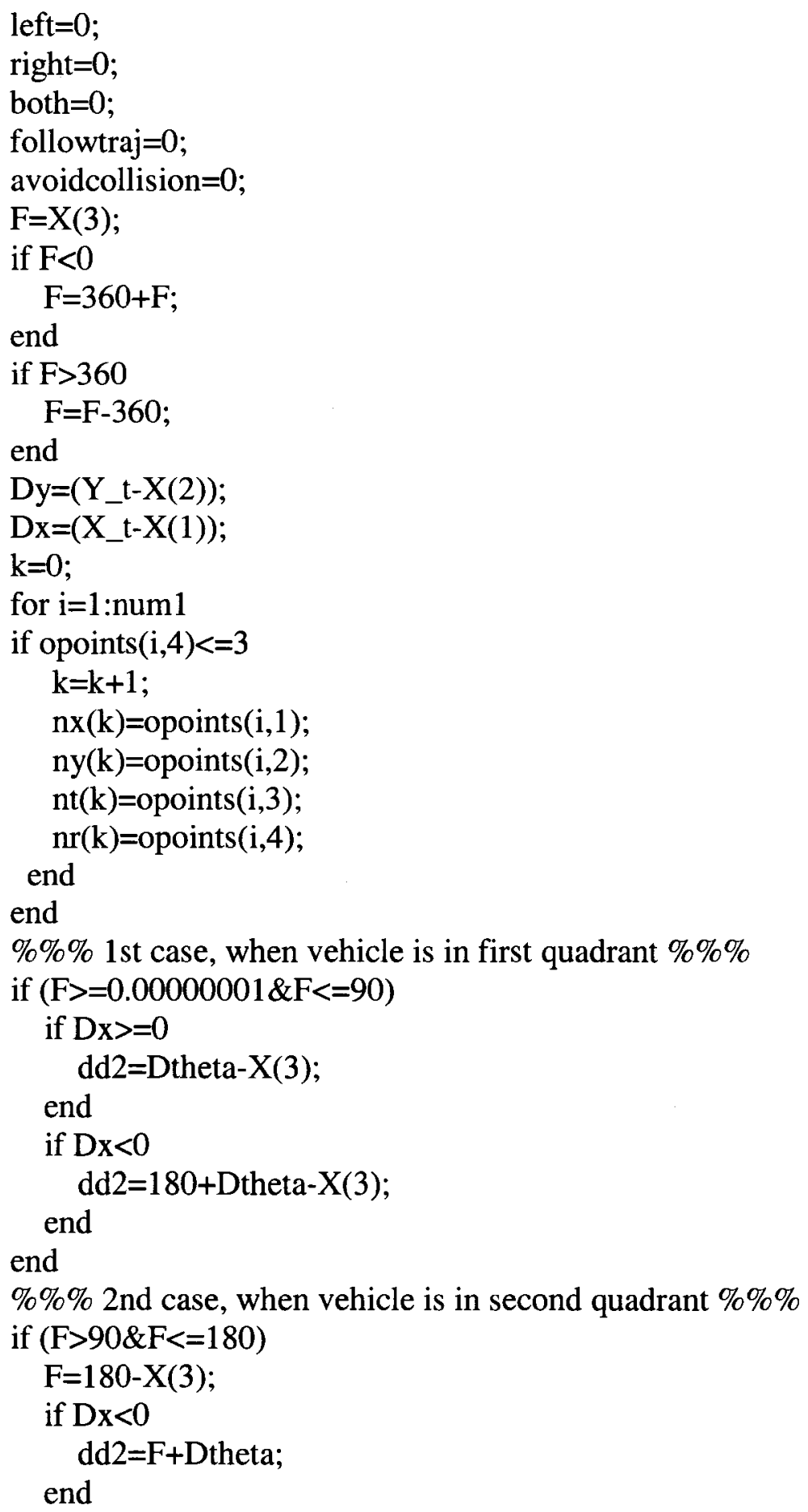




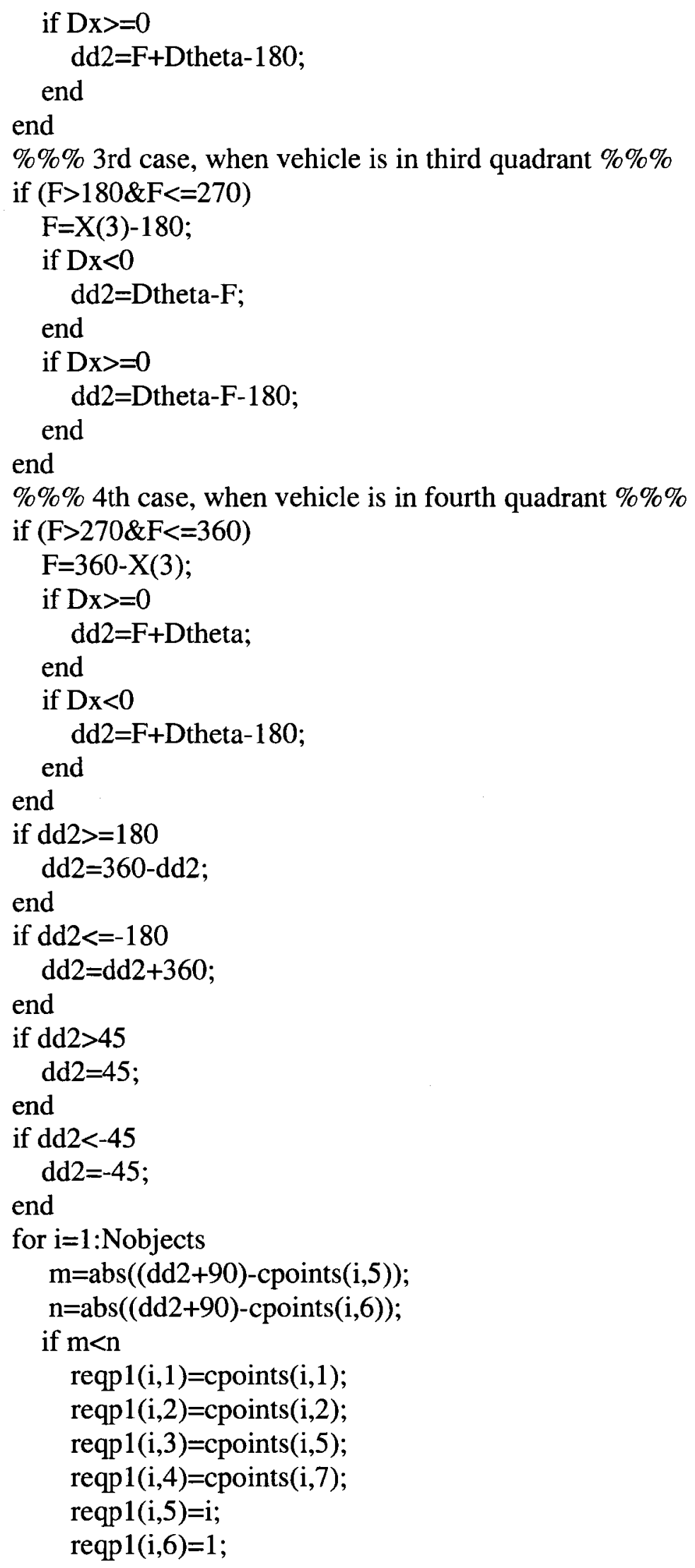




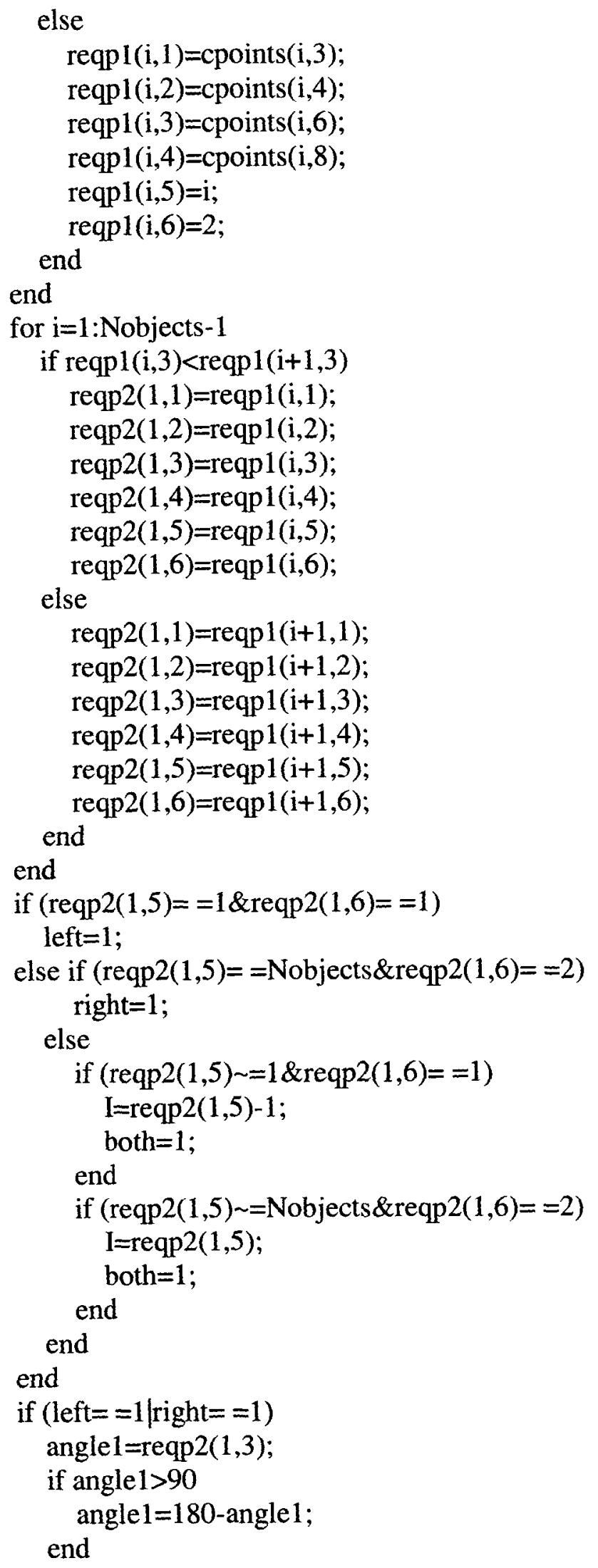




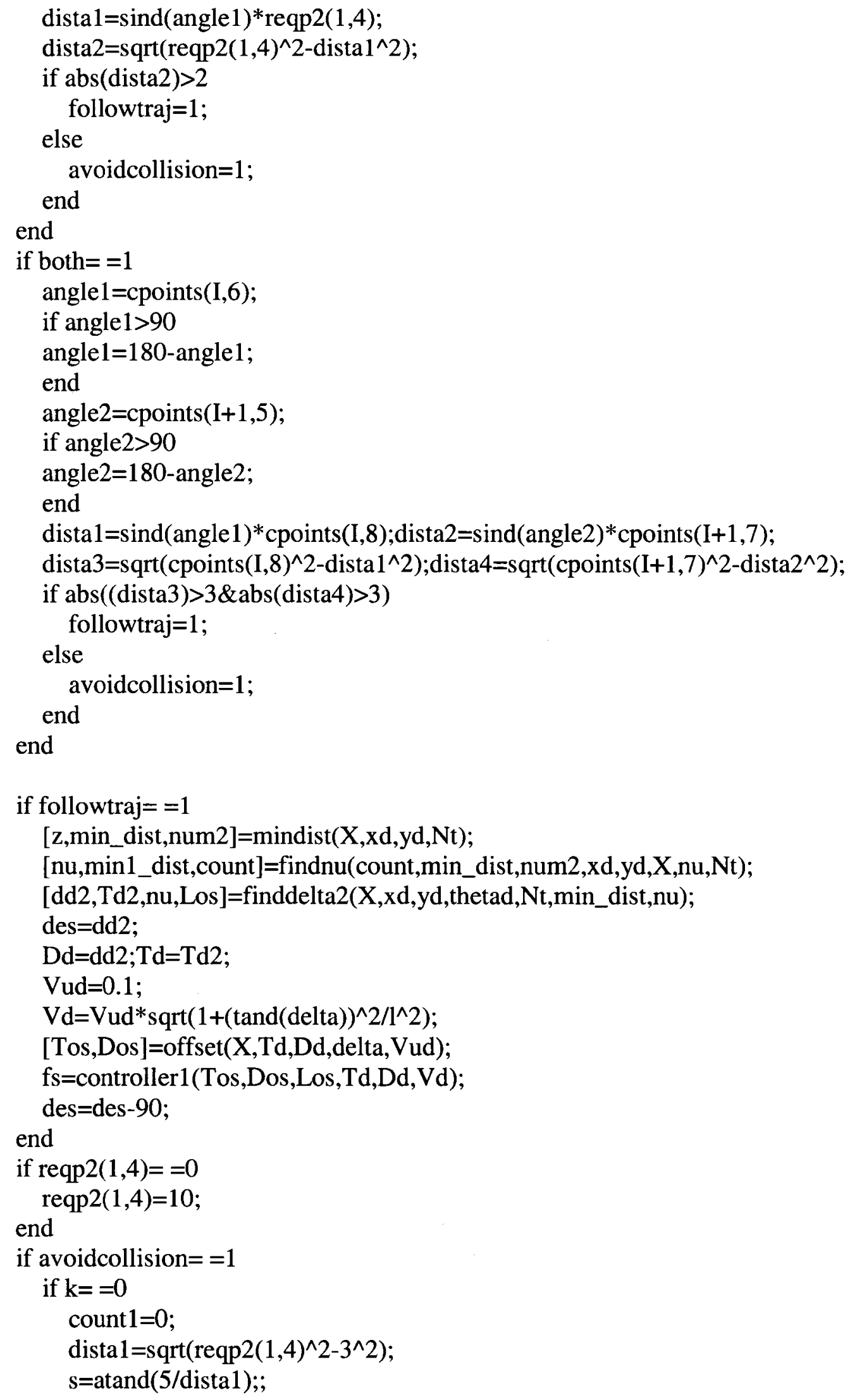




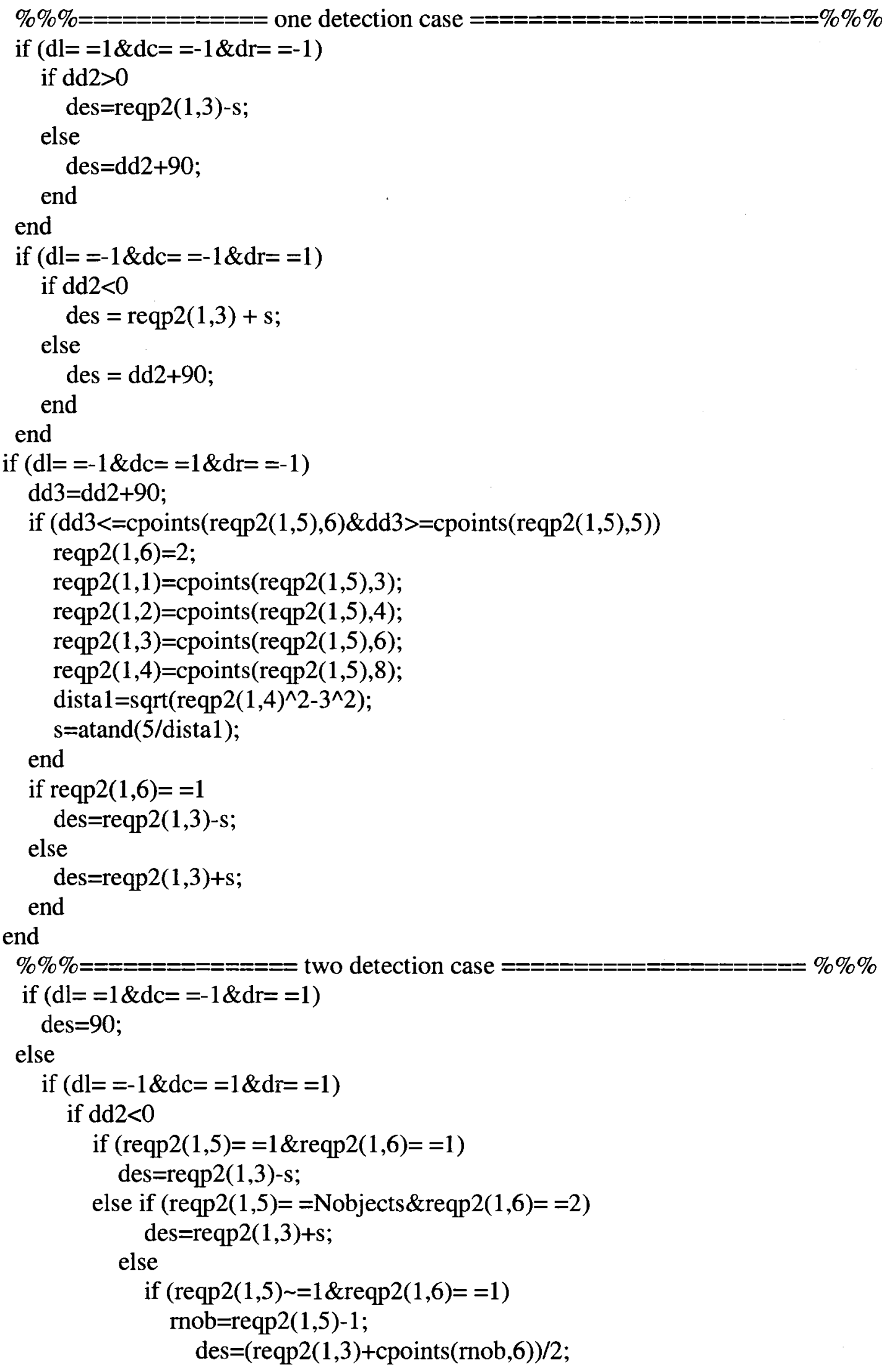




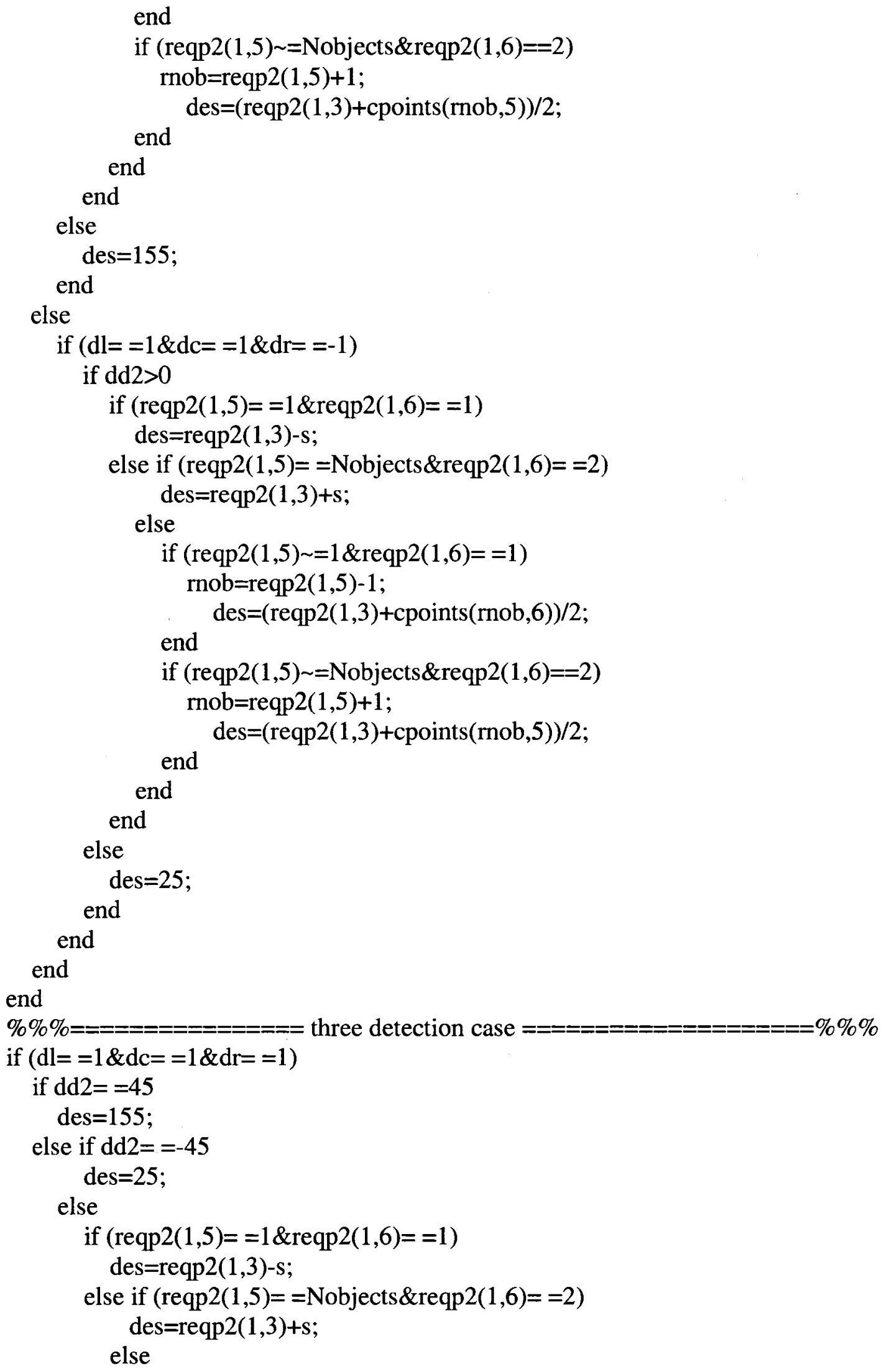




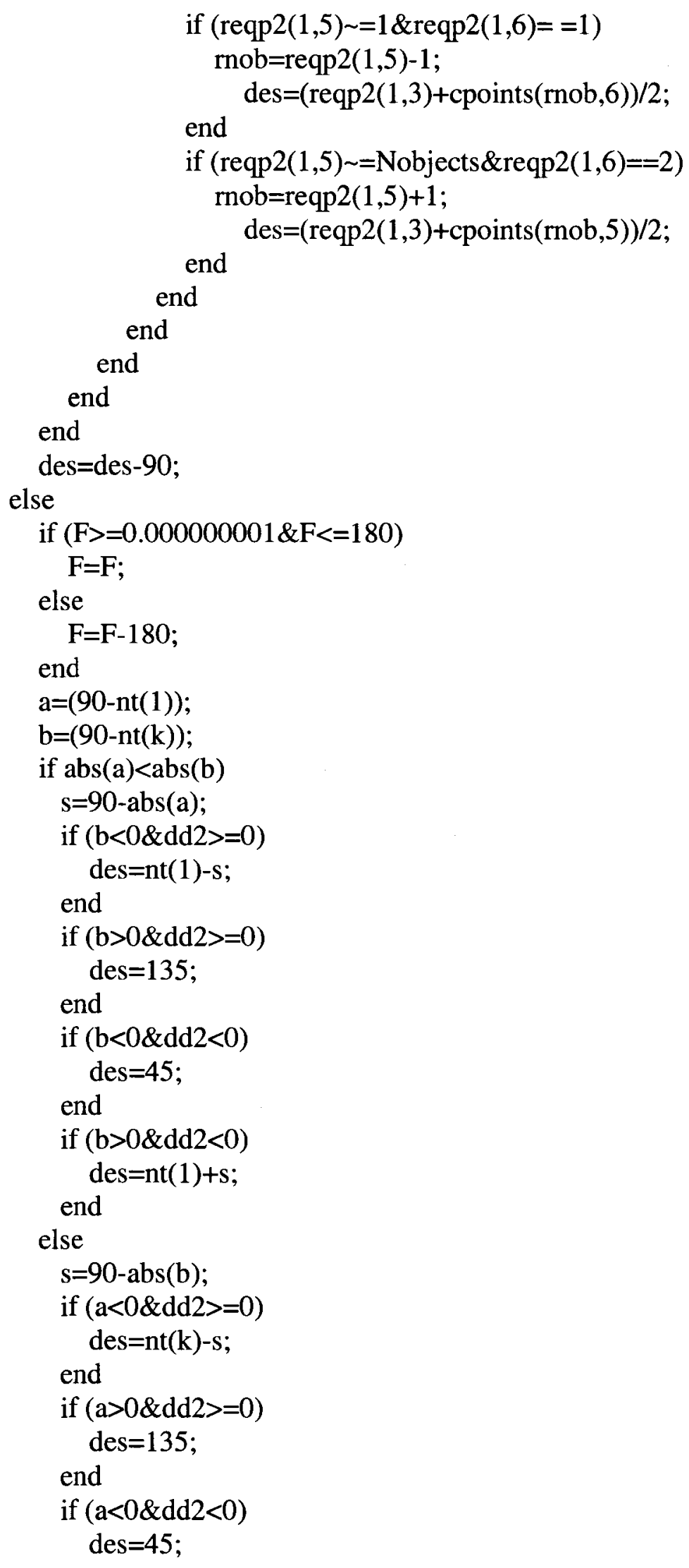




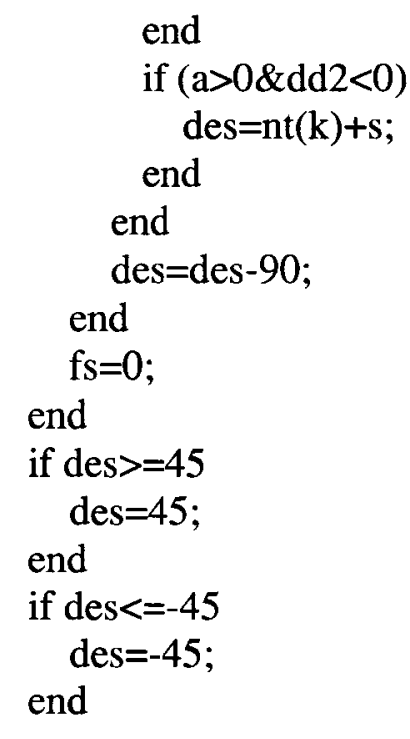

function [nu,min1_dist,count]=findnu(count,min_dist,num2,xd,yd,X,nu,Nt)

if count $==1$

nu=num2;

end

$\mathrm{Dx}=(\mathrm{xd}(\mathrm{nu})-\mathrm{X}(1))$;

if $\min \_$dist $<=5 \&$ min_dist $>4$ while $\mathrm{Dx}<0$

$\mathrm{nu}=\mathrm{nu}+1$;

if $\mathrm{nu}>\mathrm{Nt}$

$\mathrm{nu}=\mathrm{Nt}$;

break

end

$\mathrm{Dx}=(\mathrm{xd}(\mathrm{nu})-\mathrm{X}(1))$;

end

end

if min_dist $<=4$

nu=num $2+2$;

end

count $=$ count +1 ;

if $\mathrm{nu}>\mathrm{Nt}$

$\mathrm{nu}=\mathrm{Nt}$;

end

$\mathrm{nu}=\mathrm{nu}$;

$\min 1 \_$dist $=\operatorname{sqrt}\left((X(1)-x d(n u))^{\wedge} 2+(X(2)-y d(n u))^{\wedge} 2\right)$;

function [dd2,Td2,nu,Los]=finddelta2(X,xd,yd,thetad,Nt,min_dist,nu)

$\mathrm{F}=\mathrm{X}(3)$;

if $F<0$ 


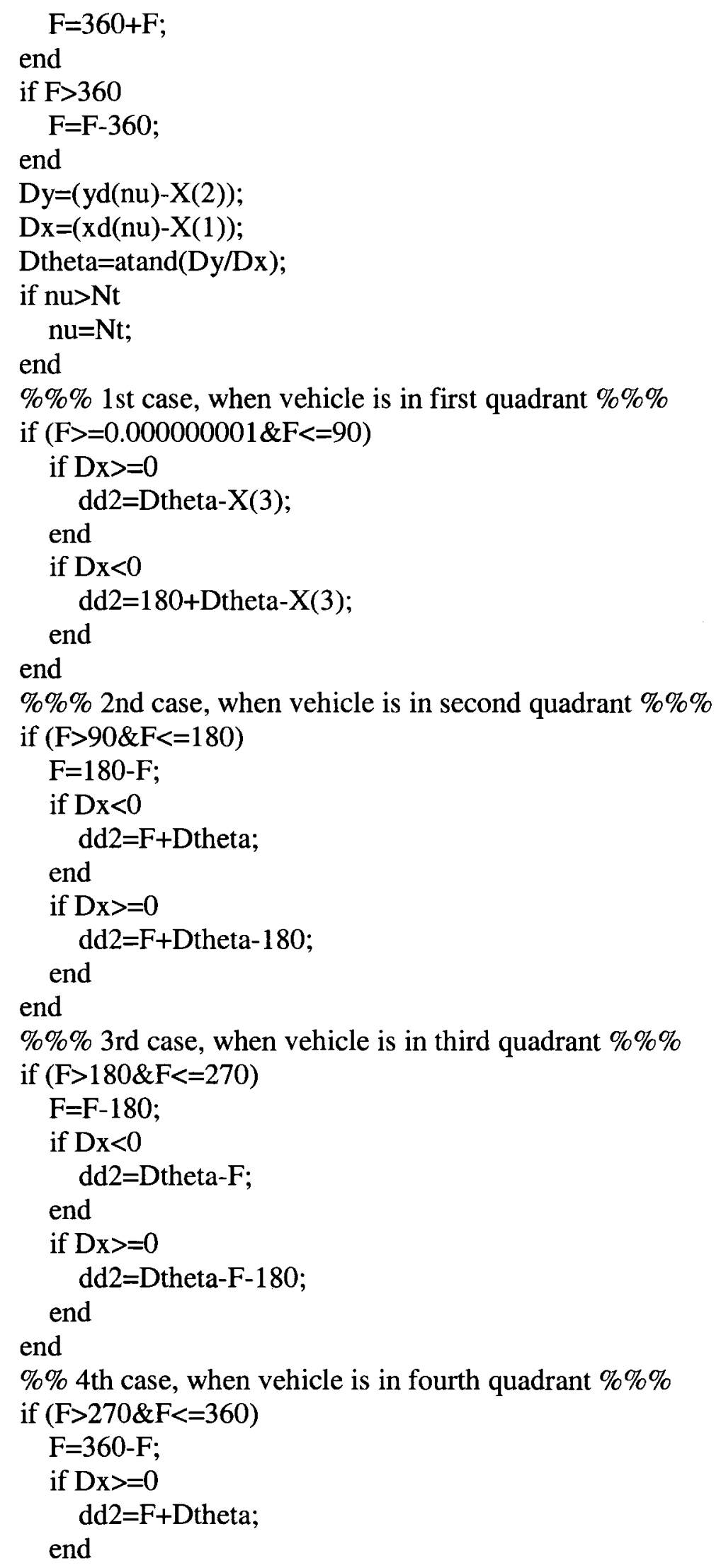




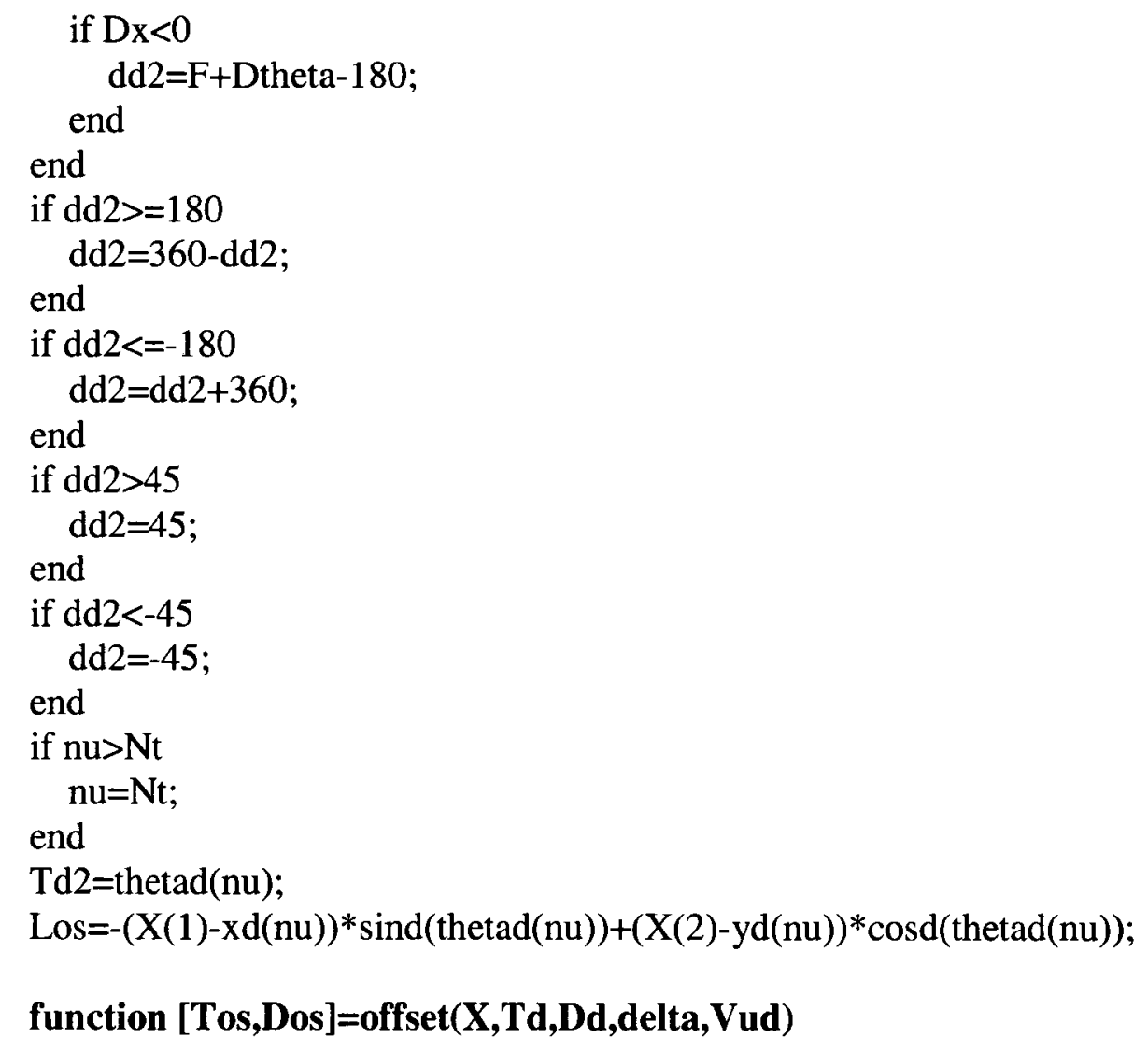

if $X(3)>=180$

$F=X(3)-360$;

end

Tos=X(3)-Td;

Dos=delta-Dd;

function fs=controller1(Tos,Dos,Los, Td,Dd,Vd)

ts $=0.1$;

Vud $=0.5$;

$\mathrm{l}=0.15$;

$\mathrm{b}=0.1$;

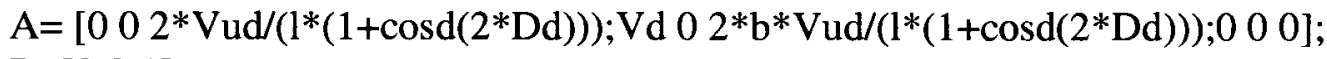

$\mathrm{B}=[0 ; 0 ; 1]$;

$\mathrm{Q}=10 * \operatorname{eye}(3)$;

$\mathrm{R}=1$;

$[\mathrm{K}, \mathrm{S}, \mathrm{e}]=\operatorname{lq}(\mathrm{A}, \mathrm{B}, \mathrm{Q}, \mathrm{R})$;

$\mathrm{fs}=-\mathrm{K}(1) * \operatorname{Tos}-\mathrm{K}(2) * \operatorname{Los}-\mathrm{K}(3) * \operatorname{Dos}$;

function fp =controller $2(\mathrm{g3}, \mathrm{g1}, \mathrm{vu}, \mathrm{fs}, \mathrm{Vud})$

$\mathrm{ts}=0.1$ 


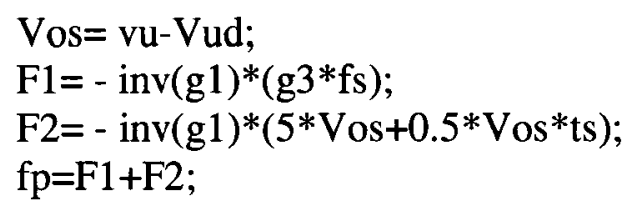

\section{function formobject(objects)}

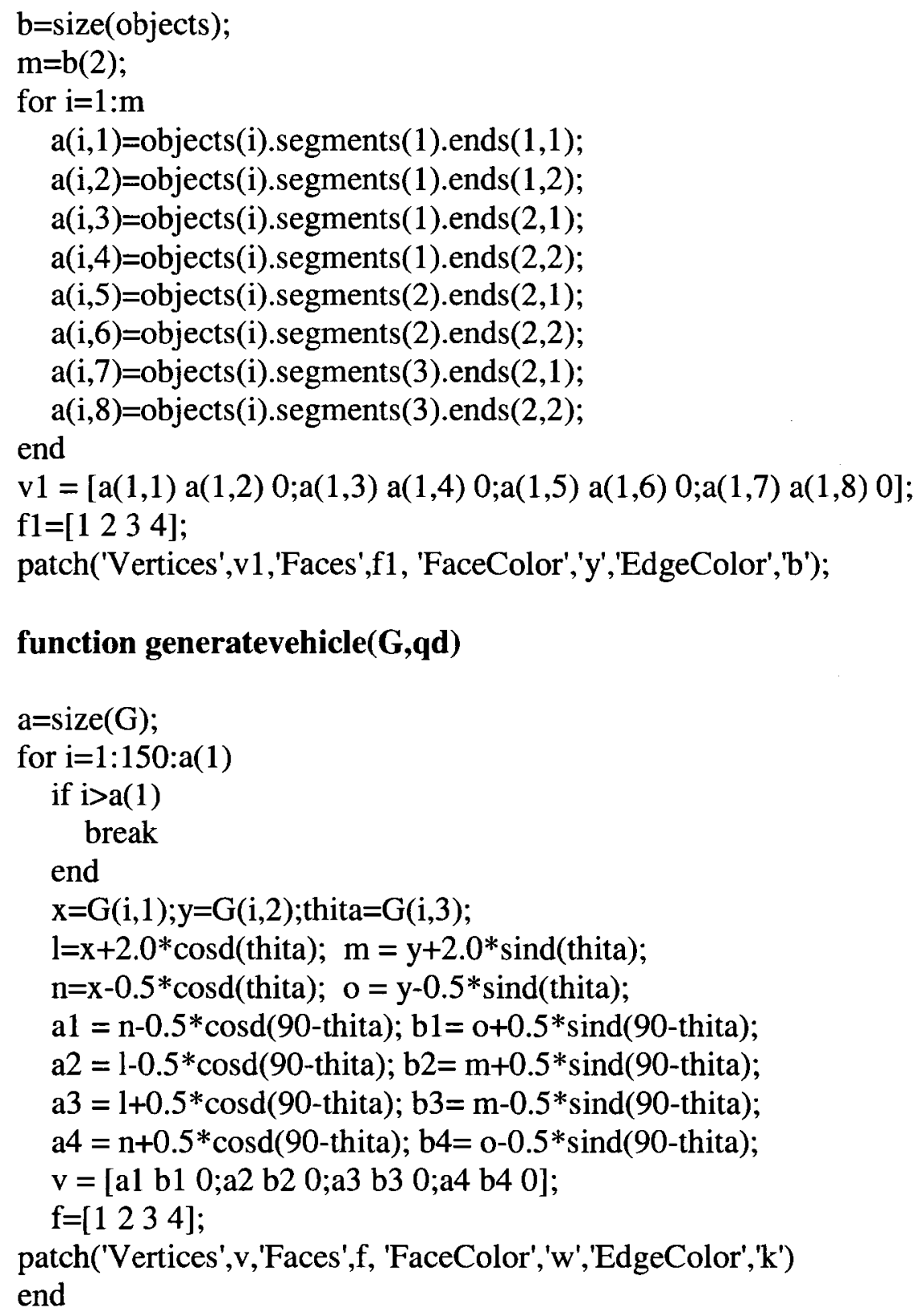

